Universidade de São Paulo

Instituto de Física

\title{
Propriedades Eletrônicas e Magnéticas de Moléculas Solvatadas
}

\author{
Rodrigo do Monte Gester
}

Tese apresentada ao Instituto de Física da USP para a obtenção do título de Doutor em Ciências

Orientador: Prof. Sylvio Roberto Accioly Canuto (IF-USP)

\section{Banca Examinadora:}

Prof. Márcio Teixeira do Nascimento Varella (IF-USP)

Prof. Antônio Carlos Borin (IQ-USP)

Prof. Roberto Rivelino de Melo Moreno (UFBA)

Prof. Benedito José da Costa Cabral (Universidade de Lisboa)

São Paulo 


\section{FICHA CATALOGRÁFICA}

\section{Preparada pelo Serviço de Biblioteca e Informação do Instituto de Física da Universidade de São Paulo}

Gester, Rodrigo do Monte

Propriedades eletrônicas e magnéticas de moléculas solvatadas. São Paulo, 2012.

Tese (Doutorado) - Universida de de São Paulo.

Instituto de Física, Depto. Física Geral.

Orientador: Prof. Dr. Sylvio Roberto Accioly Canuto

Áreade Concentração: Física Atômica e Molecular

Unitermos: 1. Físico-Química; 2. Soluções - Efeitos do solvente; 3. Simulação (Estatística) - Simulação de líquidos; 4. Ressonância magnética nuclear - Blindagem magnética e acoplamento de spins nucleares.

USP/IF/SBI-079/2012 
Aos mais velhos, Rogério, Juliana, Gester e Fátima, bem como aos mais novos, Carlos, Pedro, Luíza e Maíra, com carinho. 



\section{Sumário}

Agradecimentos viii

Abstract $\quad x$

Resumo $\quad$ xii

Publicações geradas pela Tese xiv

1 Introdução 1

$\begin{array}{lll}\text { I Teoria } & 7\end{array}$

2 Métodos teóricos em química quântica $\quad 8$

2.1 Aproximação de Hartree-Fock . . . . . . . . . . . . . . . . . . . . 8

2.2 Teoria de Perturbação M $\phi$ ller-Plesset . . . . . . . . . . . . . . . . . . 13

2.3 Teoria do funcional da densidade . . . . . . . . . . . . . . . 15

2.4 Funções base . . . . . . . . . . . . . . . . . . . . . . . . 19

3 Simulações clássicas Monte Carlo $\quad 22$

3.1 Cadeia de Markov (gerando configurações para um líquido) . . . . . . . . 23

3.2 Amostragem de Metropolis . . . . . . . . . . . . . . . . . 24

3.3 Eficiência e correlação estatística . . . . . . . . . . . . . 25

3.4 Função de distribuição radial . . . . . . . . . . . . . . . . 26

3.5 Potencial interatômico . . . . . . . . . . . . . . . . 27

4 Ressonância magnética nuclear $\quad 29$

4.1 Momento magnético nuclear . . . . . . . . . . . . . . . . 29

4.2 Níveis de energia em um campo magnético . . . . . . . . . . . . . . 30

4.3 Blindagem magnética nuclear devida aos elétrons . . . . . . . . . . . . 31

4.4 Dependência de propriedades magnéticas com relação à origem . . . . . . . 33

4.5 Acoplamento entre spins nucleares . . . . . . . . . . . . . . . . . . 34 
$5 \quad$ Uracila e 5-fluorouracila hidratadas $\quad 39$

5.1 Introdução . . . . . . . . . . . . . . . . . . . . . 39

5.2 Detalhes computacionais . . . . . . . . . . . . . . 40

5.3 Resultados e discussões . . . . . . . . . . . . . . . . . . . . 42

5.4 Conclusões . . . . . . . . . . . . . . . . . . . 52

6 Piridina hidratada $\quad 53$

6.1 Introdução . . . . . . . . . . . . . . . . . . 53

6.2 Detalhes computacionais . . . . . . . . . . . . . 54

6.3 Resultados e discussões . . . . . . . . . . . . . . . . . . 56

6.4 Conclusões . . . . . . . . . . . . . . . . . . 63

$\begin{array}{lll}7 & \text { Pirimidina hidratada } & 65\end{array}$

7.1 Introdução . . . . . . . . . . . . . . . . . . 65

7.2 Detalhes computacionais . . . . . . . . . . . . 66

7.3 Resultados e discussões . . . . . . . . . . . . . . 67

7.4 Conclusões . . . . . . . . . . . . . . . . . . . 74

8 Amônia líquida $\quad 76$

8.1 Introdução . . . . . . . . . . . . . . . . 76

8.2 Detalhes computacionais . . . . . . . . . . . . . . 77

8.3 Resultados e discussões . . . . . . . . . . . . . . . . . . 79

8.4 Conclusões . . . . . . . . . . . . . . . . . . . 9 90

9 Formamida líquida $\quad 92$

9.1 Introdução . . . . . . . . . . . . . . . . . . . . . . . . 92

9.2 Detalhes computacionais . . . . . . . . . . . . . . 93

9.3 Resultados e discussões . . . . . . . . . . . . . . . . . . . . 94

9.4 Conclusões . . . . . . . . . . . . . . . . . . . . . 107

10 Conclusões finais e perspectivas $\quad 110$ 


\section{Lista de Tabelas}

4.1 Classificação do spin nuclear segundo o números de massa e número atômico de um átomo: 30

5.1 Cargas eletrônicas $(q / e)$ e momento de dipolo $(\mu / \mathrm{D})$ calculados (MP2/aug-ccpVTZ) para uracila hidratada: . . . . . . . . . . . . . . . . 42

5.2 Cargas eletrônicas $(q / e)$ e momento de dipolo $(\mu / \mathrm{D})$ calculados (MP2/aug-ccpVTZ) para 5-fluorouracila hidratada: . . . . . . . . . . . . . . 45

5.3 Componentes isotrópicas da blindagem magnética $(\sigma / \mathrm{ppm})$ e shift gás-líquido $(\Delta \sigma / \mathrm{ppm})$ em uracila hidratada obtidas em nível de teoria mPW1PW91 (B3LYP)

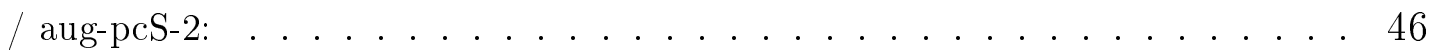

5.4 Componentes isotrópicas da blindagem magnética $(\sigma / \mathrm{ppm})$ e shift gás-líquido $(\Delta \sigma / \mathrm{ppm})$ em 5-fluorouracila hidratada. Os valores teóricos foram obtidos em nível de aproximação mPW1PW91(B3LYP)/aug-pcS-2: . . . . . . . . . . . 51

6.1 Momento de dipolo $(\mu / \mathrm{D})$ da piridina calculado em nível de teoria MP2/aug-ccpVTZ: ........................... 57

6.2 Estatística da formação de ligações de hidrogênio entre piridina e água: . . . . . 58

6.3 Efeitos da polarização do soluto e relaxação de geometria calculados sobre as constantes de blindagem magnética nuclear $\sigma\left({ }^{15} \mathrm{~N}\right)$. As constantes magnéticas $(\sigma / \mathrm{ppm})$ são calculadas em nível de aproximação B3LYP /6-311+G(d,p) enquanto as geometrias (rígida e relaxada) são obtidas com a aproximação MP2/augcc-pVTZ: ........................... 60

6.4 Blindagens magnéticas $(\sigma / \mathrm{ppm})$ calculadas em nível de aproximação B3LYP/augcc-pVTZ para o ${ }^{15} \mathrm{~N}$ em piridina hidratada: . . . . . . . . . . . . . . . 62

7.1 Parâmetros Lennard-Jones e Coulomb para pirimidina usados nas simulações Monte Carlo. Também são mostrados os momentos de dipolo (D) e cargas (e) calculados (MP2/aug-cc-PVDZ) em água. . . . . . . . . . . . . . . . 69

7.2 Estatística (\%) da formação de ligações de hidrogênio (LH) em pirimidina hidratada usando duas (PCM e iterativa) polarizações eletrônicas distintas: . . . . . . 70 
7.3 Desvio gás-água calculado sobre a componente isotrópica da blindagem magnética nuclear em pirimidina usando diferentes modelos de polarização e em nível de aproximação B3LYP/aug-pcS-2 $2^{(\mathrm{N})} / \mathrm{pcS}-2^{(\mathrm{C}, \mathrm{O}, \mathrm{H})}$. Para referência, a componente isotrópica da blindagem magnética calculada em vácuo é 91,7 ppm e desvio obtido com o modelo PCM é 15,4 ppm. . . . . . . . . . . . . . . . . 72

8.1 Momento de dipolo calculado (MP2/aug-cc-pVTZ) para amônia líquida: . . . . 79

8.2 Blindagem magnética ( $\sigma / \mathrm{ppm}$ ) calculada (B3LYP/aug-pcS-3) em amônia líquida. Valores obtidos considerando a polarização central e modelo rígido de soluto (veja

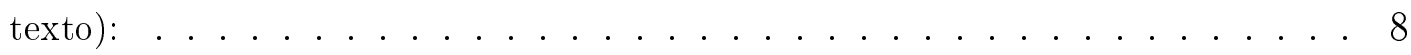

8.3 Contribuições para a blindagem magnética $(\sigma / \mathrm{ppm})$ sobre o nitrogênio considerando a participação do soluto em ligações de hidogênio: . . . . . . . . . 85

8.4 Contribuições para o acoplamento intramolecular indireto entre spins nucleares (em Hz). Todos os valores foram calculados em nível de aproximação B3LYP/augcc-pVTZ-J incluindo polarização e relaxação de geometria: . . . . . . . . . . . 87

8.5 Efeito puro da polarização (EPP) e relaxação (EPG) para o efeito total do solvente sobre as constantes de acoplamento: . . . . . . . . . . . . . . . 90

9.1 Momento de dipolo ( $\mu / \mathrm{D})$ e cargas eletrônicas (q/e) calculadas (MP2/aug-ccpVQZ) em formamida. Também são mostradas as densidades $\left(\rho / \mathrm{g}_{\mathrm{cm}} \mathrm{cm}^{-3}\right)$ obtidas com diferentes modelos de polarização eletrônica: . . . . . . . . . . . . . . . . 94

9.2 Contagem (\%) da formação de ligações de hidrogênio em formamida líquida: . 96

9.3 Blindagem magnética nuclear $(\sigma / \mathrm{ppm})$ e efeito do solvente $(\Delta \sigma / \mathrm{ppm})$ calculados sobre o oxigênio da formamida: . . . . . . . . . . . . . . . . . 98

9.4 Valores calculados [MP2/6-311 $++\mathrm{G}(d, p)]$ e empíricos para as contribuições da hidratação do oxigênio da carbonila e dos hidrogênio do grupo amida: . 101

9.5 Blindagem magnética nuclear $(\sigma / \mathrm{ppm})$ e efeito do solvente $(\Delta \sigma / \mathrm{ppm})$ calculados sobre o nitrogênio da formamida: . . . . . . . . . . . . . . . . . . 102

10.1 Resumo dos sistema moleculares estudados. Mostra-se o sentido da variação das cargas eletrônicas $\Delta q_{\mathrm{X}}$ calculadas sobre o isótopo X, bem como os desvios gás-líquido $\Delta \sigma_{\mathrm{Y}}{ }^{a}$ obtidos utilizando diferentes modelos de solvente: 111 


\section{Lista de Figuras}

4.1 Efeito Zeeman para um núcleo de momento angular $I=1$ em um campo magnético. $\quad 31$

4.2 Eleito da blindagem magnética nuclear sobre os níveis de energia Zeeman de um núcleo com momento angular $I=\frac{1}{2}$. . . . . . . . . . . . . . . . . . . . . . 32

4.3 Espectro ressonante dos prótons calculado [B3LYP /6-311 $++\mathrm{G}(d, p)]$ no álcool etílico. $\quad 33$

5.1 Uracila à esquerda. A 5-fluorouracila (à direita) é obtida partindo da uracila substituindo $\mathrm{H}_{9}$ por F. . . . . . . . . . . . . . . . . . . . . 40

5.2 Convergência do momento de dipolo calculado (MP2/aug-cc-pVTZ) para uracila (topo) e 5-fluorouracila (abaixo) hidratadas versus o número de iterações. 44

5.3 Componente isotrópica da blindagem magnética $\sigma\left({ }^{17} \mathrm{O}_{7}\right)$ em uracila hidratada. O círculos em negrito e vazados foram obtidos com os funcionais B3LYP e mPW1PW91, respectivamente. A linha tracejada corresponde ao resultado experimental. ......................... . 47

5.4 Blindagem magnética nuclear sobre o sítio $\mathrm{O}_{7}$ em função da população eletrônica. Blindagem magnética e e cargas são simultaneamente calculados em cada passo iterativo. ......................... . . 48

5.5 Convergência para o blindagem magnética nuclear $\sigma_{i s o}\left({ }^{17} \mathrm{O}_{8}\right)$ em uracila hidratada. A linha tracejada representa o valor médio 14,9,4 $\pm 1,6$ ppm obtido. . . . 48

5.6 (a) Configuração média ASEC obtida a partir das simulações clássicas MC. (b) Típica formação de ligações de hidrogênio em uracila-água. (c) Estrutura solutosolvente formando ligações de hidrogênio em água embebida no campo eletrostático das águas remanescentes. . . . . . . . . . . . . . . . 50

6.1 Piridina. ......................... 56

6.2 Momento de dipolo calculado para piridina isolada e hidratada. O círculo cheio em vermelho mostra a predição do modelo contínuo polarizado (PCM). Os pontos escuros mostram a evolução do momento de dipolo, partindo de seu valor para o sistema isolado até a convergência dessa propriedade como uma função do procedimento iterativo. ..................... 58 
6.3 Histograma da energia de interação entre pares piridina-água, obtido para o modelo rígido e polarizado. . . . . . . . . . . . . . . . 59

6.4 Estrutura piridina-água embebida no campo eletrostático das moléculas de água remanescentes. . . . . . . . . . . . . . . . . 61

6.5 Evolução da blindagem magnética $\sigma\left({ }^{15} \mathrm{~N}\right)$ calculada como uma função dos passos iterativos. Os valores foram obtidos considerando o modelo rígido. $\mathrm{O}$ dado experimental é representado pela linha pontilhada. . . . . . . . . . . . . . 62

6.6 Blindagem magnética sobre o nitrogênio, calculada em cada passo do ciclo iterativo em função da população eletrônica sobre sítio atômico. . . . . . . . . . . . 63

7.1 Pirimidina formando ligações de hidrogênio com duas moléculas de água. Os índices atômicos são usados para definir os parâmetros eletrostáticos na tabela 7.1

7.2 Valores calculados para o momento de dipolo da pirimidina hidratada usando o procedimento iterativo (I-QM/MM). Cada ponto é obtido com uma média envolvendo 100 configurações Monte Carlo. Também são mostrados os valores obtidos em vácuo (vermelho), PCM-QM/MM (círculo vazio) e PCM.

7.3 Dependência do desvio químico experimental da pirimidina em função da escala de polarização de Reichardt [193], normalizada para vinte solventes diferentes. Os valores numéricos para o desvio químico foram extraídos dos experimentos de Witanowski et al. [9], realizados em diferentes solventes: $1=$ ciclohexano, $2=\mathrm{CCl}_{4}, 3=$ benzeno, $4=\mathrm{Et}_{2} \mathrm{O}, 5=$ dioxano, $6=$ $\mathrm{CHCl}_{3}, 7=\mathrm{CH}_{2} \mathrm{C}_{12}, 8=$ acetona, $9=$ DMSO, $10=$ EtOH, $11=\mathrm{MeOH}$, $12=\mathrm{H}_{2} \mathrm{O}$.

8.1 Momento de dipolo calculado (MP2/aug-cc-pVTZ) versus o número de iterações. A curva corresponde ao modelo Impey-Klein relaxado, na qual, todas as moléculas do líquido (soluto-solvente) tiveram suas cargas atualizadas (polarização total). 80

8.2 Amônia de referência (topo) formando ligações de hidrogênio com outras duas amônias. Essa estrutura corresponde a 51\% das estruturas onde o soluto apenas aceita o hidrogênio do solvente.

8.3 Amônia de referência (topo) interagindo explicitamente com outras duas moléculas. Essa estrutura corresponde a 47,4\% das estruturas onde o soluto apenas doa o hidrogênio ao solvente.

8.4 Desvio médio gás-líquido $\Delta \sigma\left({ }^{15} \mathrm{~N}\right)$ obtido para a primeira camada de solvatação. A linha tracejada corresponde ao valor médio $-25,2 \pm 1,0$ ppm. A incerteza representa o erro estatístico. . . . . . . . . . . . . . . . 
8.5 Histograma obtido para o desvio médio gás-líquido $\Delta \sigma\left({ }^{15} \mathrm{~N}\right) . \quad \ldots \ldots . \ldots 86$

8.6 Evolução do acoplamentos indireto ${ }^{2} J(\mathrm{H}-\mathrm{H})$ com relação à polarização do soluto para o modelo Impey-Klein rígido e relaxado. Foram consideradas geometrias rígidas e relaxadas e bases aug-cc-pVTZ-J e aug-pcJ-2. . . . . . . . . . . . 8

8.7 Evolução dos acoplamento indireto ${ }^{1} J(\mathrm{~N}-\mathrm{H})$ com relação à polarização do soluto para o modelo Impey-Klein rígido e relaxado. Foram consideradas geometrias rígidas e relaxadas e bases aug-cc-pVTZ-J e aug-pcJ-2. . . . . . . . . . . . 8 8

9.1 Modelo de solvatação em amidas. $\sigma_{\text {gás }}$ representa a blindagem magnética em formamida isolada. $\sigma \mathrm{O}_{1}$ e $\sigma \mathrm{O}_{2}$ são as contribuições devidas aos lone pairs do átomo de oxigênio envolvido nas ligações de hidrogênio. $\sigma \mathrm{H}_{1}$ e $\sigma \mathrm{H}_{2}$ são as contribuições devidas à solvatação dos hidrogênio do grupo amida. . . . . . . . . . . . . . . . 99

9.2 Modelo proposto (veja texto) para explicar o aumento das blindagens magnéticas nucleares $\sigma\left({ }^{15} \mathrm{~N}\right)$ e $\sigma\left({ }^{17} \mathrm{O}\right)$ em formamida solvatada. A migração das cargas eletrônicas é motivada pela presença do solvente. Quanto maior a polaridade do meio, maior é a transferência intramolecular de cargas, gerando um efeito de shielding (aumento na blindagem). . . . . . . . . . . . . . . 103

9.3 Relação entre blindagem magnética nuclear $\sigma\left({ }^{15} \mathrm{~N}\right)$ e a carga eletrônica $q$ calculadas em cada passo do procedimento iterativo. Observe que a presença do solvente diminui a blindagem magnética sobre o núcleo de interesse ao ponto que a população eletrônica sobre o mesmo aumenta. . . . . . . . . . . . . . . . . . 104

9.4 Relação entre blindagem magnética nuclear $\sigma\left({ }^{15} \mathbf{N}\right)$ e a carga eletrônica $q$ calculadas em cada passo do procedimento iterativo em água e usando o ajuste eletrostático CHELPG. . . . . . . . . . . . . . . . . 106

9.5 Relação entre blindagem magnética nuclear $\sigma\left({ }^{15} \mathrm{~N}\right)$ e a população eletrônica calculadas em cada passo do procedimento iterativo dentro do esquema NBO (natural bond orbital). Observe que a presença do solvente diminui a blindagem magnética sobre o núcleo de interesse ao ponto que a população eletrônica sobre o mesmo aumenta. . . . . . . . . . . . . . . . . . 106

9.6 Curva de nível da densidade de blindagem magnética nuclear (DBMN). No dímero, as superfícies competem tornando o nitrogênio na posição 9 menos blindado quando comparado à formamida isolado acima. . . . . . . . . . . . . . . 107 


\section{Agradecimentos}

Não há como deixar de ser grato a todos que, de algum modo contribuíram para a realização deste trabalho. Por ocasião, gostaria de agradecer:

1. Ao CNPq pelo apoio financeiro, sem o qual não seria possível realizar este trablho.

2. Aos meus pais e irmãos, pelo apoio irrestrito ao logo desses últimos anos.

3. Aos meus amigos do Instituto de Física da USP, pelas discussões científicas e presença constante nos momentos difíceis. Em particular, agradeço a Alberto, Renato, Rafael, Lucas, Herbert, Marcus, Antônio, Bistafa, Hidalgo, Joelson, Yoelvis e Israel. Agradeço em particular a todos que passaram pela República do Gato Morto.

4. Às secretárias do DFGE, Dirce, Fátima e Silvana, que foram sempre prestativas ao longo deste processo de doutoramento.

5. À professora Kaline Coutinho pelas discussões, apoio e dicas iniciais com relação ao DICE.

6. Finalmente, gostaria de agradecer ao professor Sylvio Canuto pela inestimável colaboração e confiança depositadas em mim ao longo do doutorado. 


\section{Abstract}

Nuclear magnetic resonance is helpful on molecular characterization. Most organic molecular compounds of interest contain nitrogen or oxygen atoms which play strategic functions in chemical reactions and molecular interactions. The NMR technique provides local atomic scale information on molecular properties, thus the study of nuclear magnetic properties of these elements in molecules is of particular interest. Focusing on nuclear magnetic shielding and spin-spin coupling constants we investigated the dependence of these magnetic constants with respect to the medium. Electronic polarization and geometry relaxation effects due to solvent were systematically studied. Our general findings indicate that geometry contributions are negligible in understanding the variation of shielding constants of the investigated systems, but polarization effects are crucial for this molecular property. On nitrogen in pyridine, ammonia and formamide, for instance, isolated contributions from geometry relaxation to shielding constants are around $1.2 \mathrm{ppm}$. Nitrogen shielding constants are very sensitive to the medium, where solvent effects around $-26 \mathrm{ppm}$ are often observed. On oxygen-17 magnetic shielding, the solvent effects can easily reach $100 \mathrm{ppm}$. It is crucial to understand the origins of these effects if one desires to correctly use NMR spectroscopy for molecular characterization. Our investigations also show that magnetic shielding constants are totally independent of the nitrogen or oxygen hybridization, which indicated that the main contributions arise from solute-solvent interactions. Relevant contributions to shielding constants come from specific solute-solvent interactions like hydrogen-bonds, which can only be quantified by explicit molecular modeling, and we observed that this property has a very strong dependence on the donor or acceptor character of the atomic site involved in hydrogen-bonding interactions. On nitrogen, the acceptor behavior is responsible by the positive gas-liquid shift in shielding constants, while the donor character causes positive shift. We systematically investigated the solvent effects on indirect spin-spin coupling constants of liquid ammonia. Solute polarization is very important, but there are other fundamental contributions to this property. Without including geometry effects we calculated the ${ }^{1} J(\mathrm{~N}-\mathrm{H})$ coupling to be $-67.8 \mathrm{~Hz}$. After accounting for the solute geometry relaxation we improved the theoretical prediction obtaining the coupling constant value of $-63.9 \mathrm{~Hz}$. These results show that geometry relaxation has drastic influence on indirect spin-spin coupling constants. 


\section{Resumo}

Ressonância magnética nuclear é particularmente útil na caracterização de síntese molecular. Quase todos os compostos moleculares de interesse contêm átomos de nitrogênio e oxigênio em sua estrutura. Como esses elementos químicos costumam desempenhar funções estratégicas em reações e interações intra e intermoleculares, seus espectros NMR têm particular interesse. Com foco na blindagem magnética nuclear e no acoplamento entre spins nucleares, investigamos a dependência dessas constantes magnéticas com relação ao meio. A polarização eletrônica e relaxação geométrica do soluto, devido à presença do solvente, foram sistematicamente consideradas utilizando um tratamento sequencial QM/MM. Nossas observações gerais mostram que efeitos de relaxação de geometria mediados pelo meio têm pouca influência sobre o mecanismo da blindagem magnética nuclear, pelo menos quanto aos sistemas aqui investigados. Em contrapartida, os efeitos da polarização eletrônica são cruciais sobre essa propriedade molecular. Para o nitrogênio em piridina, amônia e formamida, por exemplo, as contribuições isoladas provenientes da relaxação geométrica são da ordem de 1,2 ppm. Note-se que este efeito é muito pequeno se considerarmos que com frequência são observados desvios gás-líquido da ordem de -26 ppm para o nitrogênio. Sobre o oxigênio, esses efeitos podem chegar até 100 ppm. Assim, é crucial entender as origens desses efeitos se desejamos utilizar corretamente espectroscopia RMN em caracterização molecular. Relevantes contribuições para a blindagem magnética têm origens em interações específicas entre soluto e solvente, como ligações de hidrogênio, as quais apenas podem ser isoladas e quantificadas através de modelagem molecular. Sistematicamente constatamos que a blindagem magnética é drasticamente afetada pelo caráter doador ou aceitador do sítio atômico envolvido em ligações de hidrogênio. Com respeito ao nitrogênio, nossos resultados indicam que o comportamento aceitador de hidrogênio de um elemento é responsável por um desvio gás-líquido positivo, enquanto que o caráter doador causa um desvio negativo. Nossas investigações também mostram que a blindagem magnética nuclear é independente com relação à hibridização do nitrogênio e oxigênio, indicando que as contribuições principais têm origens em interações específicas soluto-solvente, as quais devem ser corretamente modeladas. Investigamos sistematicamente os efeitos do solvente sobre o acoplamento indireto entre spins nucleares em amônia líquida. Embora a polarização do soluto seja realmente importante, para essa propriedade há outras contribuições fundamentais. Sem considerar efeitos de geometria, calculamos o acoplamento ${ }^{1} J(\mathrm{~N}-\mathrm{H})$ como $-67,8 \mathrm{~Hz}$. Após incluir esses efeitos, conseguimos uma descrição teórica mais apurada, obtendo um acoplamento de -63, 9 Hz. Esses resultados mostram que efeitos de relaxação geométrica mediado pelo meio têm grande influência sobre o acoplamento indireto entre spins nucleares. 


\title{
Publicações geradas pela Tese
}

Esta Tese gerou as seguintes publicações:

Rodrigo M. Gester, Herbert C. Georg, Sylvio Canuto, M. Cristina Caputo, and Patricio

F. Provasi, "NMR Chemical Shielding and Spin-Spin Coupling Constants of Liquid $\mathrm{NH}_{3}$ :

A Systematic Investigation using the Sequential QM/MM Method", Jouranl of Physical

Chemistry A, 113 (2009) 14936. doi:10.1021/jp9050484.

\begin{abstract}
The NMR spin coupling parameters, ${ }^{1} J(\mathrm{~N}, \mathrm{H})$ and ${ }^{2} J(\mathrm{H}, \mathrm{H})$, and the chemical shielding, $\sigma\left({ }^{15} \mathrm{~N}\right)$, of liquid ammonia are studied from a combined and sequential QM/MM methodology. Monte Carlo simulations are performed to generate statistically uncorrelated configurations that are submitted to density functional theory calculations. Two different Lennard-Jones potentials are used in the liquid simulations. Electronic polarization is included in these two potentials via an iterative procedure with and without geometry relaxation, and the influence on the calculated properties are analyzed. B3LYP/aug-cc-pVTZ-J calculations were used to compute the ${ }^{1} J(\mathrm{~N}, \mathrm{H})$ constants in the interval of -67.8 to $-63.9 \mathrm{~Hz}$, depending on the theoretical model used. These can be compared with the experimental results of $-61.6 \mathrm{~Hz}$. For the ${ }^{2} J(\mathrm{H}, \mathrm{H})$ coupling the theoretical results vary between -10.6 to $-13.01 \mathrm{~Hz}$. The indirect experimental result derived from partially deuterated liquid is $-11.1 \mathrm{~Hz}$. Inclusion of explicit hydrogen bonded molecules gives a small but important contribution. The vapor-to-liquid shifts are also considered. This shift is calculated to be negligible for ${ }^{1} J(\mathrm{~N}, \mathrm{H})$ in agreement with experiment. This is rationalized as a cancellation of the geometry relaxation and pure solvent effects. For the chemical shielding, $\sigma\left({ }^{15} \mathrm{~N}\right)$ calculations at the B3LYP/aug-pcS-3 show that the vapor-to-liquid chemical shift requires the explicit use of solvent molecules. Considering only one ammonia molecule in an electrostatic embedding gives a wrong sign for the chemical shift that is corrected only with the use of explicit additional molecules. The best result calculated for the vapor to liquid chemical shift $\Delta \sigma\left({ }^{15} \mathrm{~N}\right)$ is $-25.2 \mathrm{ppm}$, in good agreement with the experimental value of $-22.6 \mathrm{ppm}$.
\end{abstract}


Vinícius Manzoni, Marcelo L. Lyra, Rodrigo M. Gester, Kaline Coutinho, and Sylvio Canuto, "Study of the optical and magnetic properties of pyrimidine in water combining PCM and QM/MM methodologies", Physical Chemistry Chemical Physics, 12 (2010) 14023. doi:10.1039/c0cp00122h.

\begin{abstract}
The solvent effects on the low-lying absorption spectrum and on the ${ }^{15} \mathrm{~N}$ chemical shielding of pyrimidine in water are calculated using the combined and sequential Monte Carlo simulation and quantum mechanical calculations. Special attention is devoted to the solute polarization. This is included by an iterative procedure previously developed where the solute is electrostatically equilibrated with the solvent. In addition, we verify the simple yet unexplored alternative of combining the polarizable continuum model (PCM) and the hybrid QM/MM method. We use PCM to obtain the average solute polarization and include this in the MM part of the sequential $\mathrm{QM} / \mathrm{MM}$ methodology, PCM-MM/QM. These procedures are compared and further used in the discrete and the explicit solvent models. The use of the PCM polarization implemented in the MM part seems to generate a very good description of the average solute polarization leading to very good results for the $n-\pi^{*}$ excitation energy and the ${ }^{15} \mathrm{~N}$ nuclear chemical shield of pyrimidine in aqueous environment. The best results obtained here using the solute pyrimidine surrounded by 28 explicit water molecules embedded in the electrostatic field of the remaining 472 molecules give the statistically converged values for the low lying $n-\pi^{*}$ absorption transition in water of $36900 \pm 100$ (PCM polarization) and $36950 \pm 100 \mathrm{~cm}^{-1}$ (iterative polarization), in excellent agreement among one another and with the experimental value observed with a band maximum at $36900 \mathrm{~cm}^{-1}$. For the nuclear shielding ${ }^{15} \mathrm{~N}$ the corresponding gas-water chemical shift obtained using the solute pyrimidine surrounded by 9 explicit water molecules embedded in the electrostatic field of the remaining 491 molecules give the statistically converged values of $24.4 \pm 0.8$ and $28.5 \pm 0.8 \mathrm{ppm}$, compared with the inferred experimental value of $19 \pm 2 \mathrm{ppm}$. Considering the simplicity of the PCM over the iterative polarization this is an important aspect and the computational savings point to the possibility of dealing with larger solute molecules. This PCM-MM/QM approach reconciles the simplicity of the PCM model with the reliability of the combined QM/MM approaches.
\end{abstract}


Rodrigo M. Gester, Tertius L. Fonseca, Herbert C. Georg, Patricio F. Provasi, and Sylvio Canuto, "A simple analysis of the influence of the solvent-induced electronic polarization on the ${ }^{15}$ N magnetic shielding of pyridine in water", Theoretical Chemistry Accounts, 131 (2012) 1220. doi:10.1007/s00214-012-1220-0.

\begin{abstract}
Electronic polarization induced by the interaction of a reference molecule with a liquid environment is expected to affect the magnetic shielding constants. Understanding this effect using realistic theoretical models is important for proper use of nuclear magnetic resonance in molecular characterization. In this work, we consider the pyridine molecule in water as a model system to briefly investigate this aspect. Thus, Monte Carlo simulations and quantum mechanics calculations based on the B3LYP/ 6-311++ G(d,p) are used to analyze different aspects of the solvent effects on the ${ }^{15} \mathrm{~N}$ magnetic shielding constant of pyridine in water. This includes in special the geometry relaxation and the electronic polarization of the solute by the solvent. The polarization effect is found to be very important, but, as expected for pyridine, the geometry relaxation contribution is essentially negligible. Using an average electrostatic model of the solvent, the magnetic shielding constant is calculated as $-58.7 \mathrm{ppm}$, in good agreement with the experimental value of $-56.3 \mathrm{ppm}$. The explicit inclusion of hydrogen-bonded water molecules embedded in the electrostatic field of the remaining solvent molecules gives the value of $-61.8 \mathrm{ppm}$.
\end{abstract}




\section{Capítulo 1}

\section{Introdução}

Ressonância magnética nuclear (RMN) é um fenômeno físico no qual núcleos atômicos, sob ação de um campo magnético, absorvem e re-emitem energia. Esse efeito foi reportado pela primeira vez por Rabi [1] e, mais tarde, Bloch [2] e Purcell [3] adaptaram a técnica para o uso em sólidos e líquidos. Diferente de outras técnicas de espectroscopia, RMN não agride tanto a amostra, o que a torna uma técnica potencialmente útil na análise de compostos orgânicos [4, 5]. Essa característica tem popularizado seu uso em diagnósticos clínicos e imagens [5, 6, 7]. Em química e ciência dos materiais, RMN também é útil, permitindo determinar a estrutura molecular [5], distinguindo entre átomos, ou mesmo, isótopos que se diferenciam apenas pelo ambiente químico. É com essas aplicações, particularmente com respeito a líquidos e sistemas em vácuo que lidaremos neste texto. Nesse sentido, as constantes magnéticas do ${ }^{1} \mathrm{H}$ e ${ }^{13} \mathrm{C}$ têm sido bastante exploradas, contudo, nitrogênio e oxigênio formam compostos com quase todos os elementos, o que torna de grande interesse a estrutura e dinâmica de sistemas moleculares nitrogenados e oxigenados.

As constantes magnéticas do nitrogênio têm sido mapeadas em inúmeros trabalhos teóricos e experimentais. Sobretudo, deve-se à Witanowski e Webb [9, 10, 11, 12, 13, 14, 15] um estudo contínuo da blindagem magnética nuclear em compostos nitrogenados, como uréia, piridinas, pirimidinas, amidas, aminas e azinas. Por outro lado, há notadas dificuldades experimentais para o oxigênio. Embora seja um dos elementos mais importantes e comuns em compostos orgânicos, seu único isótopo capaz de interagir com campos magnéticos é apenas 0,037\% abundante. Essa característica faz do oxigênio um dos elementos mais difíceis de ser observado em espectroscopia RMN. Contudo, sua localização estratégica em determinadas moléculas e seu envolvimento direto em interações intra e intermoleculares, o tornam um elemento de grande interesse [16, 17].

No cenário atual, abordagens teóricas têm desempenhado papel fundamental no estudo das propriedades magnéticas nucleares em sólidos e líquidos. Há hoje uma gama 
de aproximações de química quântica desenvolvidas especificamente para o estudo de propriedades moleculares eletrônicas e magnéticas [18, 19], mas limitados pelo custo computacional, durante muito tempo o estudo teórico de sistemas moleculares ficou reduzido à investigações em vácuo. Muitos processos relevantes à química, biologia e indústria, ocorrem principalmente em ambiente aquoso, no qual, o solvente pode alterar significativamente as propriedades moleculares de interesse [8]. Com o advento dos modernos processadores, temos visto uma gama de trabalhos teóricos versando sobre a fase líquida. Modelar o ambiente aquoso não é uma tarefa simples e requer cuidados especiais. Ao contrário dos sólidos cristalinos, a fase líquida não é descrita por um potencial periódico, além do que, há constantes flutuações energéticas e rearranjos configuracionais entre os constituintes do líquido. O estado da arte em simulações computacionais utiliza um hamiltoniano totalmente quântico para descrever o sistema. Ainda nos dias atuais, abordagens como essas são muito caras e impraticáveis, limitando-se ao trato de poucas dezenas de moléculas. Uma saída seria trabalhar com um hamiltoniano híbrido, no qual apenas uma porção restrita do solvente é tratada com mecânica quântica. No tratamento $Q M / M M$ (quantum mechanics / molecular mechanics), os demais constituintes do líquido são regidos pela mecânica clássica. Do mesmo modo que em simulações totalmente quânticas, a metodologia híbrida é capaz de inferir situações que não poderiam ser acessadas por simulações clássicas, como a quebra de ligações químicas, por exemplo.

As dificuldades não se resumem somente em como obter a estrutura do líquido. Uma abordagem rigorosa dos efeitos do solvente sobre propriedades moleculares exige que cálculos de mecânica quântica sejam executados sobre o sistema composto por uma molécula de soluto, rodeada por muitas moléculas do solvente [18]. Como na fase líquida, muitas configurações são possíveis, esse procedimento deve ser administrado sobre diferentes conformações do líquido. Então, uma média sobre todas essas configurações deve ser obtida. Em muitos casos, esse procedimento é impraticável, motivando a busca por alternativas. Modelos contínuos de solvente [20,21] são uma das formas mais simples de considerar os efeitos do meio. Nessas aproximações, a estrutura do solvente é ignorada, sendo modelada como um meio dielétrico contínuo, de dimensões infinitas, com o soluto encerrado em uma cavidade imersa no dielétrico. Em modelos contínuos, o solvente é caracterizado apenas por sua constante dielétrica, obtida experimentalmente e sob condições específicas de temperatura e pressão. Em solução, a função de onda eletrônica e todas as propriedades moleculares, diferem consideravelmente de seus valores em vácuo. Isso ocorre porque a distribuição eletrônica do soluto polariza o solvente, gerando um campo elétrico chamado de campo de reação, que atua sobre o soluto. Isso significa que em implementações de modelos contínuos utilizando mecânica quântica, a relaxação da função de onda eletrônica é determinada auto-consistentemente em relação ao campo de reação do solvente. Esses 
modelos são comumente chamados de campos de reação auto-consistente [20, 21].

Há vários modelos de campos de reação, os quais diferem pela escolha do tamanho e forma da cavidade, bem como, pelo cálculo do potencial de interação soluto-solvente. No método Born-Kirkwood-Onsager [22, 23, 24], por exemplo, a cavidade é uma esfera de raio $a$ e as interações soluto-solvente são obtidas escrevendo a distribuição de cargas do soluto como um dipolo elétrico localizado no interior da cavidade. Contudo, cálculos $a b$ intio mais precisos, exigem uma forma mais realística para a cavidade. No modelo contínuo polarizado (PCM), proposto por Mertius, Scroco e Tomasi [25], essa forma é obtida centrando cada átomo do soluto em uma esfera, cujas dimensões dependem apenas do raio de van der Waals do átomo. Esse modelo tornou-se bastante popular, sofrendo evoluções ao longo dos anos. À saber, Tomasi e colaboradores têm desenvolvido o modelo IEP-PCM [26], o qual permite lidar tanto com solventes anisotrópicos, como cristais líquidos [27, 28, 29, 30], quanto com solventes isotrópicos.

Modelos contínuos exploram unicamente interações eletrostáticas, ignorando a forma do solvente. Por isso costumam falhar quando outras interações entre soluto e solvente são necessárias, como ligações de hidrogênio, por exemplo. Outras abordagens foram pensadas para lidar com situações em que o solvente precisa ser incluído explicitamente, mas obter um hamiltoniano quântico, ou mesmo híbrido, ainda é um problema. O tratamento sequencial Mecânica Quântica / Mecânica Molecular (s-QM/MM) é uma dessas metodologias. Neste procedimento, primeiro as configurações do líquido são geradas por meio de simulações clássicas, em seguida, cálculos de mecânica quântica são executados sobre essas configurações a fim de obter as propriedades médias de interesse. Como simulações clássicas são computacionalmente mais baratas do que seus pares quânticos, a proposta sequencial tornou-se uma importante ferramenta no trato de sistemas moleculares de grande porte. Como exemplo, um tratamento sequencial, no qual a parte clássica é descrita por meio de simulações Monte Carlo (MC), tem sido desenvolvido e utilizado no estudo de propriedades moleculares de compostos de interesse químico e biológico $[31,32,33]$.

Constantes magnéticas envolvendo elementos como o nitrogênio e oxigênio são bastante sensíveis ao meio, assim, um olhar mais detalhado sobre esses parâmetros pode determinar quais interações são fundamentais em ressonância magnética. Buckingham e colaboradores $[34,35]$ têm racionalizado os efeitos do solvente em contribuições aditivas das interações de van der Waals e eletrostática. Nessa proposta, interações específicas, como ligações de hidrogênio, por exemplo, seriam classificadas como um tipo de interação eletrostática forte. Trabalhos recentes [36, 37] mostram que ligações de hidrogênio soluto-solvente têm papel crucial em RMN, mas uma boa descrição teórica do experimento somente é obtida após considerar o campo eletrostático das moléculas mais 
afastadas. Está claro que ligações de hidrogênio figuram entre as mais importantes interações em líquidos moleculares [8, 38, 39], contudo, há outras contribuições fundamentais. Witanowski e colaboradores $[9,10,11,12,13,14,15]$, por exemplo, têm ressaltado a dependência da blindagem magnética $\sigma\left({ }^{15} \mathrm{~N}\right)$ com relação à polaridade do solvente. Também recentemente, tem-se observado extrema dependência de muitas propriedades, inclusive magnéticas, com respeito à polarização eletrônica do soluto [36, 40, 41, 42, 43, 44, 45, 46].

Os fatos discutidos acima sugerem que uma atenção especial precisa ser dispensada à polarização eletrônica induzida pelo meio. Entender esses efeitos utilizando modelos realísticos é crucial para o uso adequado da RMN em caracterização molecular. Fora de simulações totalmente quânticas, ou mesmo híbridas, há hoje duas formas de inferir os efeitos da polarização devido ao meio. Modelos contínuos são, sem dúvida, a maneira mais simples. A segunda consiste em administrar um procedimento iterativo [47, 48], o qual permite equilibrar as cargas eletrônicas do soluto na presença do solvente. Esse procedimento tem sido utilizado para inferir os efeitos do solvente sobre diversas propriedades moleculares, como os espectros de absorção eletrônica [42, 43, 47, 48] e RMN [36, 45], por exemplo. O esquema iterativo utiliza simulações clássicas, Monte Carlo ou dinâmica molecular, para gerar as configurações do líquido. Em seguida, essas estruturas são sobrepostas, gerando uma configuração representativa média, na qual o solvente é representado apenas pelas cargas eletrônicas localizadas em suas respectivas posições nucleares. Em seguida, essa configuração média é usada para recalcular a nova distribuição de cargas eletrônicas do soluto. Essas cargas são, então, reimplementadas em uma nova simulação clássica e o processo é repetido até a convergência do momento de dipolo molecular.

Utilizamos modelos contínuos, discretos e explícitos de solvente para investigar os efeitos do meio sobre propriedades magnéticas nucleares em líquidos moleculares. A primeira parte do texto compreende os quatro primeiros capítulos, nos quais são discutidos os métodos e aproximações computacionais utilizados para obter as propriedades de interesse. Os cinco capítulos seguintes discutem os resultados e conclusões obtidas para os sistemas investigados.

No capítulo 5, investigamos os efeitos do solvente sobre a blindagem magnética nos oxigênios da uracila e 5-fluorouracila hidratadas. Dispensamos especial atenção à polarização eletrônica do soluto e ao papel da formação de ligações de hidrogênio no líquido. Na descrição de propriedades magnéticas nucleares, a teoria atual afirma que o solvente deve ser considerado explicitamente nos cálculos e que interações provenientes do bulk também devem ser incorporadas. De fato, nossos melhores resultados são obtidos para estruturas soluto-solvente eletronicamente polarizadas, submetidas ao campo elétrico das moléculas remanescentes. Com essa aproximação, obtivemos uma excelente correlação teoria-experimento, com desvios da ordem de $3 \%$ em relação ao experimento. Contudo, 
utilizando o modelo da configuração eletrostática média do solvente (ASEC) [47, 48], também obtivemos descrições que diferem da observação experimental por valore inferiores à $5 \%$. Considerando a complexidade em descrever também o solvente com funções de onda, o modelo da configuração eletrostática ASEC, representa uma alternativa em potencial no trato de sistemas maiores. De modo geral, o efeito do solvente é dramático sobre a blindagem magnética $\sigma\left({ }^{17} \mathrm{O}\right)$, para a qual obtivemos desvios gás-água de até 112 ppm, mostrando considerável sensibilidade ao meio.

No capítulo 6 , discutimos os efeitos do meio sobre a blindagem magnética do nitrogênio em piridina hidratada. Isso inclui especialmente os efeitos de relaxação geométrica e polarização eletrônica induzidos pelo solvente. A polarização eletrônica mostrou-se fundamental, mas como esperado para piridina, efeitos geométricos são irrelevantes. Novamente a melhor descrição foi obtida utilizando modelos que acoplam interações explícitas solute-solvente às interações eletrostáticas de longo alcance provenientes das moléculas remanescentes, contudo, com o modelo eletrostático médio de solvente [47, 48], discutido acima, obtivemos blindagens magnéticas nucleares $\sigma\left({ }^{15} \mathrm{~N}\right)$ com desvios de apenas $4,3 \%$ em relação ao experimento.

No capítulo 7, discutimos a blindagem magnética sobre o nitrogênio-15 em pirimidina hidratada. Como em uracila e 5-fluorouracila, utilizamos uma alternativa ainda inexplorada, a qual concilia a simplicidade de modelos contínuos e a metodologia QM/MM sequencial. Através do modelo PCM, obtivemos a polarização eletrônica do soluto e a implementamos na parte clássica do tratamento QM/MM. Com esse modelo, denominado PCM-QM/MM, obtivemos um desvio gás-água de 24,4 \pm 0, 8 ppm sobre o nitrogênio da pirimidina, em boa concordância com o valor experimental de $19 \pm 2 \mathrm{ppm}$.

No capítulo 8, discutimos a blindagem magnética $\sigma\left({ }^{15} \mathrm{~N}\right)$ e os acoplamentos ${ }^{1} J(\mathrm{~N}-\mathrm{H})$ e ${ }^{2} J(\mathrm{H}-\mathrm{H})$ em amônia líquida. Além da polarização eletrônica, inferimos efeitos de relaxação geométrica sobre essas constantes. Nossos melhores resultados, novamente conciliam interações diretas entre soluto e solvente à contribuições eletrostáticas. Como em piridina, os efeitos da polarização eletrônica devido ao meio são fundamentais sobre a blindagem magnética em nitrogênio, mas os efeitos de relaxação geométrica são desprezíveis. Para os acoplamentos ${ }^{1} J(\mathrm{~N}-\mathrm{H})$ e ${ }^{2} J(\mathrm{H}-\mathrm{H})$, contrariamente, os efeitos de geometria são fundamentais, com magnitudes de até 3, $4 \mathrm{~Hz}$.

No capítulo 9, investigamos as blindagens magnéticas em formamida líquida. Para o oxigênio-17, em vácuo, obtivemos uma blindagem de $-35,7$ ppm, em boa descrição do valor experimental de $-35,5$ ppm [49]. Para o líquido homogêneo, nosso melhor modelo prediz uma blindagem de 22, 9 ppm, em boa correlação com o experimento, o qual reporta 24, 0 ppm [50]. Revisitamos os modelos de solvatação de amidas propostos por Burgar, Amou e Fiat [51, 52] e Gerothanassis e Vaaka [16, 55, 56]. Nossos resultados mostram que é 
possível discutir a blindagem magnética total sobre o oxigênio em termos de contribuições provenientes de outros sítios moleculares que não o oxigênio. Isso implica que, em amidas, a blindagem $\sigma\left({ }^{17} \mathrm{O}\right)$ não é uma propriedade local. Constatamos que a solvatação dos hidrogênios do grupo amida, a qual antes se pensava irrelevante para $\sigma\left({ }^{17} \mathrm{O}\right)$, tem papel crucial. Os modelos de Burgar, Amou e Fiat e Gerothanassis são aqui sistematicamente revistos, primeiro considerando a estatística da formação de ligações de hidrogênio no líquido, bem como efeitos da polarização eletrônica induzida pelo meio. Em um segundo momento, inclui-se interações eletrostáticas com as moléculas afastadas do soluto.

Na descrição das constantes magnéticas do nitrogênio-15 em formamida, também obtivemos boa correlação com o experimento. Para o líquido homogêneo, nossa melhor estimativa prediz uma blindagem de 147,3 ppm, em boa aproximação do valor experimental de 149,2 pm [50]. Não tivemos acesso à dados experimentais para o gás, mas estimamos uma blindagem de 173,1 ppm. Nossos resultados mostram um considerável efeito do solvente de $-25,8$ ppm. O sinal negativo no desvio gás-líquido chama atenção. Pelo modelo proposto por Witanowski [12, 14], um dos principais efeitos do solvente seria a migração eletrônica de cargas no soluto. O modelo proposto implica que o acúmulo de cargas sobre o sítio atômico, necessariamente demanda um aumento na blindagem magnética nuclear do elemento e que esse efeito é potencializado pela polaridade do solvente. Diferente dos sistemas que discutimos até aqui, formamida e amônia líquidas não são explicadas por esse modelo. Embora a polarização eletrônica induza um aumento na população eletrônica nos nitrogênios, a blindagem magnética $\sigma\left({ }^{15} \mathrm{~N}\right)$ diminui nestes compostos, quando em solução. Nossos resultados indicam que o fator preponderante para a blindagem magnética não é o acúmulo de cargas eletrônicas sobre o elemento de interesse. 


\section{Parte I}

\section{Teoria}




\section{Capítulo 2}

\section{Métodos teóricos em química quântica}

Há uma extensa gama de métodos de estrutura eletrônica desenvolvidos para o estudo de propriedades atômicas e moleculares. Mesmo que feita de maneira qualitativa, a discussão de todos esses métodos seria inviável no âmbito que se propõe esse trabalho. Algumas dessas aproximações, como o método de Huckel e outros métodos semi-empíricos, tiveram seu papel e motivações bem definidos na história da ciência. Foram largamente utilizados não somente por sua eficácia no estudo da estrutura eletrônica, mas também por representarem alternativas de baixo custo computacional. Com o desenvolvimento dos modernos computadores, aproximações mais sofisticadas e que antes não poderiam ser utilizadas no estudo dos mais simples sistemas atômicos, hoje são corriqueiramente empregadas no trato de sistemas moleculares em geral. Dentre essas, podemos citar o método Hartree-Fock, a teoria do funcional da densidade, bem como os métodos pós Hartree-Fock. Serão discutidas agora apenas as aproximações de química quântica utilizadas aqui para obter as propriedades de interesse.

\subsection{Aproximação de Hartree-Fock}

\section{Considerações iniciais}

Na teoria do orbital molecular a dupla ocupação eletrônica de um orbital espacial $\phi$ pode ser obtida fazendo

$$
\chi_{2 i}(p)=\phi_{i}(p) \alpha(\uparrow) \quad \text { e } \quad \chi_{2 i-1}(q)=\phi_{i}(q) \beta(\downarrow)
$$

onde as funções $\left\{\phi_{i}\right\}$ representam as componentes espaciais do estado $i$, sendo ainda $\alpha$ e $\beta$ suas contribuições do spin eletrônico up $(\uparrow)$ e down $(\downarrow)$, respectivamente. Finalmente $\chi$ são os spin-orbitais e são dados pelos produtos das componentes espacial e de spin.

Para um sistema de $N$ elétrons, a função de onda total anti-simétrica é dada por um 
determinante de Slater $\Psi$ :

$$
\Psi=\frac{1}{\sqrt{N !}}\left|\begin{array}{cccc}
\phi_{1}(1) & \phi_{1}(2) & \ldots & \phi_{1}(N) \\
\phi_{2}(1) & \phi_{2}(2) & \ldots & \phi_{2}(N) \\
\vdots & \vdots & \ddots & \vdots \\
\phi_{N}(1) & \phi_{N}(2) & \ldots & \phi_{N}(N)
\end{array}\right|
$$

Uma maneira simples de construir o determinante $\Psi$ consiste em utilizar o produto de Hartree obter todas as contribuições anti-simétricas executando uma série de permutações sobre os orbitais espaciais [53]. Ou seja:

$$
\Psi=\frac{1}{\sqrt{N !}} \sum_{P}(-1)^{l} P\left\{\phi_{1}(1) \phi_{2}(2) \cdots \phi_{N}(N)\right\}
$$

onde o operador $P$ engloba todas as permutações de entre 1, 2 até $N$ corpos. O termo $(-1)^{l}$ assume valores +1 ou -1 para permutações pares ou ímpares, respectivamente.

Dentro da aproximação de Born-Oppenheimer [53, 54], a qual desacopla os movimento eletrônico e nuclear, o hamiltoniano totalmente eletrônico de um sistema molecular é escrito como

$$
\hat{H}=\sum_{p} h_{p}=-\frac{1}{2} \sum_{p} \nabla_{p}^{2}-\sum_{p, A} \frac{Z_{A}}{r_{p A}}+\frac{1}{2} \sum_{p, q} r_{p q}^{-1}
$$

Determinar uma expressão para a energia eletrônica $E_{0}$ é um problema que está diretamente ligado ao cálculo dos valores médios $\langle\Psi|\hat{H}| \Psi\rangle$. É possível mostrar que a expressão da energia eletrônica é dada por [54]:

$$
E_{0}=2 \sum_{i} h_{i i}+\sum_{i, j}\left(2 J_{i j}-K_{i j}\right)
$$

onde a soma é feita sobre os estados e não sobre as partículas. $h_{i i}$ é dado por

$$
h_{i i}=\int \phi_{i}^{*}(1) h(1) \phi_{i}(1) d \mathbf{r}_{1}
$$

e compreende todas as integrais de um elétron. $J_{i j}$ e $K_{i j}$ são integrais de dois corpos, sendo usualmente definidas em termos dos operadores de Coulomb $\hat{J}_{i}(1)$ e troca $\hat{K}_{i}(1)$ :

$$
J_{i j}=\int \phi_{i}^{*}(1) \hat{J}_{j}(1) \phi_{i}(1) d \mathbf{r}_{1}
$$

e

$$
K_{i j}=\int \phi_{i}^{*}(1) \hat{K}_{j}(1) \phi_{i}(1) d \mathbf{r}_{1}
$$

A integral $J_{i j}$ define as interações coulombianas entre as densidades eletrônicas $\left|\phi_{i}\right|^{2}$ e $\left|\phi_{j}\right|^{2}$. 
Por outro lado, $K_{i j}$ não tem análogo clássico, sendo conhecido apenas como integral de troca. Os operadores $\hat{J}_{i}(1)$ e $\hat{K}_{i}(1)$ são por sua vez definidos como:

$$
\hat{J}_{j}(1)=\int \phi_{j}^{*}(2) r_{12}^{-1} \phi_{j}(2) d \mathbf{r}_{2}
$$

e

$$
\hat{K}_{j}(1) \phi_{i}(1)=\left[\int \phi_{j}^{*}(2) r_{12}^{-1} \phi_{i}(2) d \mathbf{r}_{2}\right] \phi_{j}(1)
$$

Os operadores $h_{i i}, J_{i j}$ e $K_{i j}$ são hermitianos e permitem escrever as integrais de Coulomb e troca como:

$$
J_{i j}=\int \phi_{i}^{*}(1) \hat{J}_{j}(1) \phi_{i}(1) d \mathbf{r}_{1}=\int \phi_{j}^{*}(1) \hat{J}_{i}(1) \phi_{j}(1) d \mathbf{r}_{1}
$$

e

$$
K_{i j}=\int \phi_{i}^{*}(1) \hat{K}_{j}(1) \phi_{i}(1) d \mathbf{r}_{1}=\int \phi_{j}^{*}(1) \hat{K}_{i}(1) \phi_{j}(1) d \mathbf{r}_{1}
$$

\section{Equações de Hartree-Fock}

Embora a forma funcional da energia eletrônica $E_{0}$ para um sistema de camadas fechadas seja conhecida, há algumas complicações. Os operadores que definem a energia eletrônica $E_{0}$ são escritos em termos dos orbitais espaciais $\phi_{i}$ que compõem o determinante de Slater $\Psi$. Isso é um problema pois o conjunto $\left\{\phi_{i}\right\}$ ainda é desconhecido. Em tese, qualquer base pode ser usada na representação dos orbitais espaciais. Contudo, precisa-se definir uma critério de escolha. Nesta seção será discutido o método do campo auto-consistente de Hartree-Fock, o qual usa o princípio variacional para determinar o melhor conjunto de funções de onda espaciais possível.

A ideia consiste em minimizar a energia eletrônica $E_{0}$ variando as funções $\phi_{i}$ e exigindo que sua ortogonalidade $\left[\int \phi_{i}^{*}(1) \phi_{j}(1) d \mathbf{r}_{1}=\mathbf{1}\right]$ seja respeitada. Ou seja:

$$
\delta E=\delta E_{0}-2 \sum_{i, j} \varepsilon_{j i} \delta\left[\int \phi_{i}^{*}(1) \phi_{j}(1) d \mathbf{r}_{1}\right]=0
$$

onde $-2 \varepsilon_{j i}$ é o multiplicador de Lagrange. O fator -2 foi escolhido apenas para tornar mais fáceis os cálculos que seguem. Como os orbitais moleculares são ortogonais, há a variação sobre $\phi_{i}$ dada por:

$$
\int \delta \phi_{i}^{*}(1) \phi_{j}(1) d \mathbf{r}_{1}+\int \phi_{i}^{*}(1) \delta \phi_{j}(1) d \mathbf{r}_{1}=0
$$


Usando as relações $(2.5),(2.6),(2.11),(2.12),(2.13)$ e (2.14), têm-se então ${ }^{1}$ :

$2 \int \delta \phi_{i}^{*}(1)\left\{\hat{h}(1)+\sum_{j}\left[2 \hat{J}_{j}(1)-\hat{K}_{j}(1)\right]\right\} \phi_{i}(1) d \mathbf{r}_{1}-2 \sum_{j} \varepsilon_{j i} \int \delta \phi_{i}^{*}(1) \phi_{j}(1) d \mathbf{r}_{1}+c . c .=0$

A solução não trivial implica que o integrando deve anular-se. Desse modo serão geradas duas equações dadas por:

$$
\hat{h}(1) \phi_{i}(1)+\sum_{j}\left[2 \hat{J}_{j}(1)-\hat{K}_{j}(1)\right] \phi_{i}(1)-\sum_{j} \varepsilon_{j i} \phi_{j}(1)=0 \quad \text { e } \quad c . c .=0
$$

Como $h_{i i}, J_{i j}$ e $K_{i j}$ são operadores hermitianos, a diferença entre as duas expressões acima é:

$$
\sum_{j} \phi_{j}\left(\varepsilon_{j i}-\varepsilon_{i j}^{*}\right)=0
$$

As funções $\phi_{i}$ são ortogonais, isso implica que também são linearmente independentes. Desse modo:

$$
\varepsilon_{j i}=\varepsilon_{i j}^{*}
$$

Pode-se definir o operador de Fock $F$ como:

$$
\hat{F}(1)=\hat{h}(1)+\sum_{j}\left[2 \hat{J}_{j}(1)-\hat{K}_{j}(1)\right]
$$

como isso, a eq.(2.16) é reescrita como um problema de auto-valores e auto-vetores na forma:

$$
\hat{F} \phi_{i}=\sum_{j} \phi_{j} \varepsilon_{j i}
$$

A equação acima é conhecida como equação de Hartree-Fock. Como o operador $F$ também é escrito em termos dos orbitais espaciais $\phi_{i}$, a solução dessa equação só pode ser obtida de modo auto-consistente. Inicialmente uma função $\phi_{i}^{(1)}$ é usada para construir o operador $\hat{F}^{(1)}$. Em seguida o problema de auto-valores e auto-vetores é resolvido para $\varepsilon_{i}^{(2)}$ e $\phi_{i}^{(2)}$, dados na equação abaixo.

$$
\hat{F}^{(1)} \phi_{i}^{(2)}=\varepsilon_{i}^{(2)} \phi_{i}^{(2)} \quad i=1,2,3, \ldots, N / 2
$$

onde $N$ é o número elétrons do sistema. A nova solução $\phi_{i}^{(2)}$ é usada para obter um novo operador $\hat{F}^{(2)}$. O processo é repetido ciclicamente até que as energias convirjam

\footnotetext{
${ }^{1}$ Como os índices nas somas são mudos, a igualdade $\left\langle\delta \phi_{i}^{*} \phi_{j}^{*}\left|O_{2}\right| \phi_{i} \phi_{j}\right\rangle=\left\langle\phi_{i}^{*} \delta \phi_{j}^{*}\left|O_{2}\right| \phi_{i} \phi_{j}\right\rangle$ vale para qualquer operador $\mathrm{O}_{2}$ genérico. Isso justifica o fator 2 que aparece multiplicando a eq.(2.15).
} 
para um valor $\varepsilon_{i}^{(n)}$. Esse processo iterativo é conhecido como ciclo auto-consistente de Hartree-Fock.

\section{Equações de Hartree-Fock-Roothaan}

A simetria esférica simplifica consideravelmente o uso do método Hartree-Fock no tratamento de sistemas atômicos. Para moléculas em geral, há quebra de simetria esférica e as soluções das equações (2.21) tornam-se complicadas do ponto de vista numérico. Roothaan resolveu esse problema propondo que as funções de onda moleculares fossem expandidas em uma combinação linear de orbitais atômicos (LACO-MO ${ }^{2}$ ), ou método de Hartree-Fock-Roothaan [57]. O método consiste em tomar a expansão:

$$
\phi_{i}=\sum_{\mu=1}^{K} C_{\mu i} \varphi_{\mu} \quad i=1,2, \ldots, K
$$

onde $\phi_{i}$ são os já conhecidos orbitais moleculares e $\varphi_{i}$ são os orbitais atômicos. Se $\left\{\varphi_{i}\right\}$ é um conjunto completo, a solução é exata. Contudo, por razões computacionais apenas um número finito $(K)$ de funções bases é usado. O problema anterior de calcular os orbitais moleculares usando o método Hartree-Fock, agora se resume a determinar o conjunto de coeficientes $C_{\mu i}$ para os quais a equação de Hartree-Fock é satisfeita. Desse modo, substituindo a expansão acima na eq.(2.21), têm-se:

$$
\hat{F}(1) \sum_{\mu} C_{\mu i} \varphi_{\mu}(1)=\varepsilon_{i} \sum_{\mu} C_{\mu i} \varphi_{\mu}(1)
$$

Multiplicando toda a expressão por $\varphi_{\nu}^{*}(1)$ e integrando sobre as coordenadas espaciais da partícula 1, obtém-se a forma integral:

$$
\sum_{\mu} C_{\mu i} \int \varphi_{\nu}^{*}(1) \hat{F}(1) \varphi_{\mu}(1) d \mathbf{r}_{1}=\varepsilon_{i} \sum_{\mu} C_{\mu i} \int \varphi_{\nu}^{*}(1) \varphi_{\mu}(1) d \mathbf{r}_{1}
$$

onde costuma-se definir as matrizes

$$
S_{\nu \mu}=\int \varphi_{\nu}^{*}(1) \varphi_{\mu}(1) d \mathbf{r}_{1} \quad \text { e } \quad F_{\nu \mu}=\int \varphi_{\nu}^{*}(1) \hat{F}(1) \varphi_{\mu}(1) d \mathbf{r}_{1}
$$

$S_{\nu \mu}$ é uma matriz hermitiana, usualmente chamada de matriz de cobrimento. Embora os orbitais atômicos sejam normalizados, não são necessariamente ortogonais. Isso faz com que a matriz $S_{\nu \mu}$ não seja em geral nula. A matriz de Fock $F_{\nu \mu}$ também é uma matriz hermitiana quadrada de ordem $K$. Em termos das matrizes $F_{\nu \mu}$ e $S_{\nu \mu}$ definidas acima,

\footnotetext{
${ }^{2}$ A sigla LCAO-MO vem do inglês, Linear Combination of Atomic Orbital - Molecular Orbital
} 
reescreve-se a equação de Hartree-Fock como:

$$
\sum_{\mu} F_{\nu \mu} C_{\mu i}=\varepsilon_{i} \sum_{\mu} S_{\nu \mu} C_{\mu i}
$$

Essa última expressão é conhecida como equação de Hartree-Fock-Roothaan, que em notação matricial é escrita como:

$$
\mathrm{FC}=\mathrm{SC} \varepsilon
$$

As matrizes $\mathrm{C}$ e $\boldsymbol{\varepsilon}$ também são de ordem $K$, sendo dadas por:

$$
\mathbf{C}=\left(\begin{array}{cccc}
C_{11} & C_{12} & \cdots & C_{1 K} \\
C_{21} & C_{22} & \cdots & C_{2 K} \\
\vdots & \vdots & \ddots & \vdots \\
C_{K 1} & C_{K 2} & \cdots & C_{K K}
\end{array}\right) \text { e } \boldsymbol{\varepsilon}=\left(\begin{array}{cccc}
\varepsilon_{11} & & & \\
& \varepsilon_{22} & & \mathbf{0} \\
\mathbf{0} & & \ddots & \\
& & & \varepsilon_{K K}
\end{array}\right)
$$

O método Hartree-Fock e LCAO-MO representam uma forma eficiente de tratar sistemas atômicos e moleculares. Contudo, há algumas considerações que devem ser observadas. Tanto Hartree-Fock quanto e LCAO-MO são métodos iterativos que possibilitam encontrar o melhor conjunto de orbitais moleculares $\phi_{i}$ que construirão o determinante de Slater $\Psi$. O determinante de Slater é obtido extraindo as componentes antissimétricas do produto de Hartree.

No aproximação LCAO-MO, interações elétron-elétron são representadas pelo operador

$$
G=\sum_{j}\left(2 J_{j}-K_{j}\right)
$$

o qual descreve esses efeitos na forma de um campo médio. Isso significa que o elétron não interage diretamente com todos os demais elétrons, mas sim com seu o campo médio. Usualmente definida como a diferença entre a energia exata $E_{0}$ não relativística e a energia Hartree-Fock $E_{H F}$, a energia de correlação eletrônica $E_{c o r r}$ é de fundamental importância. Normalmente são usados métodos perturbativos pós Hartree-Fock para tratar a correlação eletrônica. Na próxima seção discutiremos um desses métodos.

\subsection{Teoria de Perturbação M $\phi$ ller-Plesset}

Em se tratando de cálculos ab initio em estrutura eletrônica, as quatro causas de erros são $(a)$ o tratamento inadequado da correlação eletrônica, $(b)$ a incompleteza do conjunto de funçães base, $(c)$ efeitos relativísticos e, $(d)$ desvios devido à aproximação de Born- 
Oppenheimer. Efeitos relativísticos são relevantes para sistemas moleculares que incluem elementos pesados, por isso, não serão discutidos aqui. Desvios devido à aproximação de Born-Oppenheimer são usualmente negligenciáveis para moléculas no estado fundamental. Portanto, para a maioria dos casos, $(a)$ e $(b)$ são as duas maiores fontes de erros.

O método Hartree-Fock negligencia os efeitos da correlação eletrônica. Contudo, podemos contar com uma gama de aproximações quânticas pensadas para incluir esses efeitos. Métodos perturbativos estão entre as aproximações mais populares. Em 1934, M $\phi$ ller e Plesset (MP) desenvolveram uma aproximação para átomos e moléculas no qual a função de onda não perturbada é a função de onda Hartree-Fock, o qual discutiremos agora. A abordagem se restringirá à moléculas de camadas fechadas e no estado fundamental, como os sistemas que investigamos.

A teoria perturbativa MP se baseia na aproximação Rayleigh-Shrödinger [54], mas com o hamiltoniano não perturbado, $H_{0}$, escrito como uma soma de operadores de Fock $\hat{F}(\mu)[58]$

$$
\hat{H}_{0}=\sum_{\mu} \hat{F}(\mu)=\sum_{\mu} \hat{h}(\mu)+\sum_{\mu} \sum_{j}\left[2 \hat{J}_{j}(\mu)-\hat{K}_{j}(\mu)\right]
$$

A potencial perturbativo $V$ é, portanto, a diferença entre o hamiltoniano molecular $H$ verdadeiro e $H_{0}$. Com isso

$$
\hat{V}=\hat{H}-\hat{H}_{0}=\sum_{\mu} \sum_{\nu>\mu} \frac{1}{r_{\mu \nu}}-\sum_{\mu} \sum_{j}\left[2 \hat{J}_{j}(\mu)-\hat{K}_{j}(\mu)\right] .
$$

Note-se que a perturbação $\hat{V}$ é a diferença entre um operador de repulsão (correlação) eletrônica e os operadores de Fock, os quais são obtidos usando uma teoria de campo médio.

A correção perturbativa de primeira ordem $E_{0}^{1}$ para a energia do estado fundamental é dada por $E_{0}^{(1)}=\left\langle\phi_{0}^{(0)}|\hat{V}| \phi_{0}^{(0)}\right\rangle$, onde os índices inferiores e superiores denotam, respectivamente, a função de onda do estado fundamental e a ordem de perturbação. Até a primeira ordem de perturbação, temos então

$$
E_{0}^{(0)}+E_{0}^{(1)}=\left\langle\phi_{0}^{(0)}\left|\hat{H}_{0}+\hat{V}\right| \phi_{0}^{(0)}\right\rangle=\left\langle\phi_{0}^{(0)}|H| \phi_{0}^{(0)}\right\rangle .
$$

Note-se que $\left\langle\phi_{0}^{(0)}|\hat{H}| \phi_{0}^{(0)}\right\rangle$ é a integral variacional para a função de onda Hartree-Fock $\phi_{0}^{(0)}$, que é, portanto, igual à energia Hartree-Fock $E_{H F}$. Em consequência

$$
E_{0}^{(0)}+E_{0}^{(1)}=E_{H F}
$$


O resultado acima mostra que para obter melhores descrições do sistema é necessário considerar ordens superiores de perturbação. Para correções de segunda ordem da energia eletrônica, tem-se que

$$
E_{0}^{(2)}=\sum_{n \neq 0} \frac{\left|\left\langle\phi_{n}^{(0)}|\hat{V}| \phi_{0}^{(0)}\right\rangle\right|^{2}}{E_{0}^{(0)}-E_{n}^{(0)}} .
$$

Considere a matriz $\left\langle\phi_{n}^{(0)}|\hat{V}| \phi_{0}^{(0)}\right\rangle$. Pelo teorema de Brillouin [53] excitações simples $\left(\left\langle\Phi_{i}^{a}|V| \phi_{0}^{(0)}\right\rangle\right)$ anulam essa integral. Pelas regras de seleção Condon-Slater [54], também têm contribuições nulas excitações de ordens superiores ou igual a três. Assim, só precisamos nos preocupar com determinantes duplamente excitados, $\Phi_{i j}^{a b}$.

Ao tomar as energias moleculares $E_{0}^{(0)}+E_{0}^{(1)}+E_{0}^{(2)}=E_{H F}+E_{0}^{(2)}$, temos uma aproximação comumente designada como MP2, na qual, o índice 2 indica a inclusão de correções de segunda ordem para a energia.

\subsection{Teoria do funcional da densidade}

A função de onda de um sistema de $n$-elétrons depende de $3 n$ coordenadas espaciais e $n$ coordenadas de spin. Se considerarmos o fato de que o hamiltoniano eletrônico é composto por operadores de apenas um e dois elétrons, está claro que a função de onda eletrônica contem mais informação do que necessário para calcular as propriedades moleculares de interesse, tornando os cálculos de estrutura eletrônica mais caros. Esse fato tem motivado a busca por funções que dependam de menos variáveis para o cálculo da energia eletrônica e outras propriedades.

\section{Teorema de Hohenberg-Kohn}

Hohenberg e Kohn [59] mostraram que, para moléculas com estado fundamental não degenerado, a energia eletrônica e todas as demais propriedades moleculares são unicamente determinadas pela densidade de probabilidade eletrônica $\rho_{0}(\mathbf{r})$ do estado fundamental, a qual é uma função de apenas três variáveis.

A função de onda eletrônica é um auto valor do hamiltoniano eletrônico

$$
\hat{H}=-\frac{1}{2} \sum_{i} \nabla_{i}^{2}+\sum_{i} v\left(\mathbf{r}_{i}\right)+\sum_{i} \sum_{j>i} \frac{1}{r_{i j}},
$$

no qual, o potencial externo $v\left(\mathbf{r}_{i}\right)=-\sum_{K} \frac{Z_{K}}{r_{i K}}$ depende apenas das coordenadas eletrônicas em relação ao núcleo. 
Com o potencial externo e o número de elétrons especificados, os estados eletrônicos e suas respectivas energias são determinados resolvendo a equação de Schrödinger. Hohenber e Kohn [59] mostraram que, para sistemas com estado fundamental não degenerado, a densidade eletrônica $\rho_{0}(\mathbf{r})$, também determina o potencial externo $v\left(\mathbf{r}_{i}\right)$, bem como o número de elétrons.

Para mostrar que $\rho_{0}(\mathbf{r})$ determina o número de elétrons do sistema, basta integrá-la em todas as coordenadas espaciais para obter $\int \rho_{0}(\mathbf{r}) d \mathbf{r}=n$. A prova de que o potencial externo pode ser determinado pela densidade eletrônica é demostrada por absurdo. Considere que há dois potenciais externos diferentes, $v_{a}\left(\mathbf{r}_{i}\right)$ e $v_{b}\left(\mathbf{r}_{i}\right)$, capazes de gerar a mesma densidade eletrônica $\rho_{0}(\mathbf{r})$ e, que diferem por apenas por uma constante. Note-se que esses potenciais externos definem, respectivamente, as hamiltinianas $\hat{H}_{a}$ e $\hat{H}_{b}$, as quais têm como auto-valores, as auto-energias $E_{0, a}$ e $E_{0, b}$. Note-se que as auto-funções, $\psi_{0, a}$ e $\psi_{0, b}$ são diferentes, uma vez que, as hamiltonianas também diferem entre si por mais de uma constante.

Se o estado fundamental não é degenerado, há somente uma função de onda $\psi_{0}$ relacionada à energia exata $E_{0}$. Pelo princípio variacional, se tentarmos utilizar qualquer outra função de onda, obteremos energias maiores que $E_{0}$. Isso significa que $\langle\phi|\hat{H}| \phi\rangle>E_{0}$. Desse modo, temos que

$$
E_{0, a}<\left\langle\psi_{0, b}\left|\hat{H}_{a}\right| \psi_{0, b}\right\rangle=\left\langle\psi_{0, b}\left|\hat{H}_{a}+\hat{H}_{b}-\hat{H}_{b}\right| \psi_{0, b}\right\rangle
$$

Como os hamiltonianos diferem apenas pelos potencias externos, tem-se que $\hat{H}_{a}-\hat{H}_{b}=$ $\sum_{i}\left[v_{a}\left(\mathbf{r}_{i}\right)-v_{b}\left(\mathbf{r}_{i}\right)\right]$ e, portanto

$$
E_{0, a}-E_{0, b}<\int \rho_{0, b}(\mathbf{r})\left[v_{a}(\mathbf{r})-v_{b}(\mathbf{r})\right] d \mathbf{r},
$$

na qual, a densidade eletrônica $\rho_{0, b}(\mathbf{r})$ foi explicitada. Se o processo for refeito considerando agora $E_{0, b}<\left\langle\psi_{0, a}\left|\hat{H}_{b}\right| \psi_{0, a}\right\rangle$, tem-se que

$$
E_{0, b}-E_{0, a}<\int \rho_{0, a}(\mathbf{r})\left[v_{b}(\mathbf{r})-v_{a}(\mathbf{r})\right] d \mathbf{r} .
$$

Por hipótese, consideramos que as duas funções de onda fornecem a mesma densidade eletrônica. Ao fazer $\rho_{0, a}=\rho_{0, b}$ nas duas desigualdades acima, vê-se que $E_{0, a}+E_{0, b}<$ $E_{0, a}+E_{0, b}$, o que é um absurdo. Logo, dois potenciais externos diferentes não podem produzir a mesma densidade eletrônica. Isso significa que a densidade de probabilidade eletrônica do estado fundamental $\rho_{0}$ determina o potencial externo e, como vimos, também o número de elétrons. Em consequência, $\rho_{0}$ também determina a hamiltoniana do sistema, bem como a função de onda, energia e demais propriedades eletrônicas.

A energia do estado fundamental $E_{0}$ pode ser escrita como um funcional da densidade 
eletrônica. Desse modo, $E_{0}=E_{v}\left[\rho_{0}\right]$. O hamiltoniano puramente eletrônico é dado pela soma das contribuições das energias cinética, elétron-elétron e elétron-núcleo. Dentro formalismo DFT, para sistema no estado fundamental, tem-se então

$$
E_{0}=E_{v}\left[\rho_{0}\right]=T\left[\rho_{0}\right]+V_{e N}\left[\rho_{0}\right]+V_{e e}\left[\rho_{0}\right]
$$

no qual, $\hat{V}_{e N}=\sum_{i} v\left(\mathbf{r}_{i}\right)$. Com isso, o funcional $V_{e N}\left[\rho_{0}\right]$ pode ser reescrito em termos da densidade eletrônica

$$
V_{e N}\left[\rho_{0}\right]=\left\langle\psi_{0}|\hat{V}| \psi_{0}\right\rangle=\int \rho_{0}(\mathbf{r}) v(\mathbf{r}) d \mathbf{r} .
$$

Assim, embora o funcional $V_{e N}\left[\rho_{0}\right]$ seja conhecido, há informações sobre $T\left[\rho_{0}\right]$ e $V_{e e}\left[\rho_{0}\right]$. Contudo, pode-se sempre definir um funcional $F\left[\rho_{0}\right]=T\left[\rho_{0}\right]+V_{e e}\left[\rho_{0}\right]$ independente do potencial externo. Com isso, tem-se a energia eletrônica

$$
E_{0}=E_{v}\left[\rho_{0}\right]=\int \rho_{0}(\mathbf{r}) v(\mathbf{r}) d \mathbf{r}+F\left[\rho_{0}\right]
$$

Note-se que a equação acima ainda não fornece uma forma prática de calcular a energia $E_{v}$ partindo da densidade $\rho_{0}$, uma vez que o funcional $F\left[\rho_{0}\right]$ ainda não é conhecido.

\section{Teorema variacional de Hohenberg e Kohn}

Para contornar o problema do funcional $F\left[\rho_{0}\right]$ na equação anterior, são necessários somente o segundo teorema de Hohenberg e Kohn [59], em uma aproximação desenvolvida por Kohn e Sham [60]. O segundo teorema diz que para qualquer função densidade $\rho_{t}(\mathbf{r}) \geq 0$ que satisfaça a condição $\int \rho_{t}(\mathbf{r}) d \mathbf{r}=n$, sempre teremos que $E_{0} \leq E_{v}\left[\rho_{t}\right]$. Desde que $E_{0}=E_{v}\left[\rho_{0}\right]$, então a densidade eletrônica do estado fundamental minimiza o funcional $E_{v}\left[\rho_{t}\right]$. Pelo primeiro teorema de Hohenberg e Kohn, $\rho_{t}$ determina o potencial externo $v_{t}(\mathbf{r})$, que por sua vez determina a função de onda $\Psi_{t}$, que corresponde à densidade $\rho_{t}$. Com isso, $\left\langle\psi_{t}|\hat{H}| \psi_{t}\right\rangle \geq E_{0}$ torna-se

$$
\left\langle\psi_{t}\left|\hat{T}+\hat{V}_{e e}+\sum_{i} v(\mathbf{r})\right| \psi_{t}\right\rangle \geq E_{0}=E_{v}\left[\rho_{0}\right]
$$

ou, finalmente

$$
T\left[\rho_{t}\right]+V_{e e}\left[\rho_{t}\right]+\int \rho_{t}(\mathbf{r}) d \mathbf{r}=E_{v}\left[\rho_{t}\right] \geq E_{v}\left[\rho_{0}\right]
$$

Como $E_{v}\left[\rho_{t}\right] \geq E_{v}\left[\rho_{0}\right]$, está provado que nenhuma outra função densidade teste $\rho_{t}$ pode fornecer uma energia menor que a densidade eletrônica $\rho_{0}$ do estado fundamental. 


\section{Equações de Kohn-Sham}

Uma vez conhecida a densidade eletrônica $\rho_{0}$ do estado fundamental, os teoremas de Hohenber e Kohn nos dizem que é possível calcular todas as propriedades moleculares no estado fundamental partindo de $\rho_{0}$. Contudo, não nos dizem como calcular a energia $E_{0}$, uma vez que o funcional $F\left[\rho_{0}\right]$ é desconhecido, nem mesmo como encontrar $\rho_{0}$ sem que primeiro obter a função de onda eletrônica. A solução para o problema foi encontrada em 1965 por Kohn e Sham [60]. Embora a DFT, como descrita nas seções anteriores, seja uma teoria exata, a implementação das equações de Khon-Sham (KS) produz resultados aproximados.

Kohn e Sham adotaram como referência um sistema fictício, um gás de partículas não interagentes sob ação de um potencial externo $v_{s}\left(\mathbf{r}_{i}\right)$, o qual é unicamente determinado pela densidade eletrônica $\rho_{s}(\mathbf{r})$. Note-se que a densidade $\rho_{s}(\mathbf{r})$ é escolhida de tal modo à reproduzir a densidade eletrônica $\rho_{0}$ do sistema interagente de interesse. Os elétrons do gás não interagem, desse modo, o hamiltoniano eletrônico do sistema de referência é escrito como

$$
\hat{H}_{s}=\sum_{i}\left[-\frac{1}{2} \nabla_{i}^{2}+v_{s}\left(\mathbf{r}_{i}\right)\right]=\sum_{i} h_{i}^{\mathrm{KS}},
$$

no qual, $h_{i}^{\mathrm{KS}}$ é o hamiltoniano monoeletrônico de Kohn-Sham. Como o sistema de referência $s$ é constituído de partículas não interagentes, as soluções do hamiltoniano de KohnSham são um produto antissimetrizado dos spin-orbitais $u_{i}=\phi_{i}^{\mathrm{KS}} \sigma_{i}$. Desse modo

$$
h_{i}^{\mathrm{KS}} \phi_{i}^{\mathrm{KS}}=\varepsilon_{i}^{\mathrm{KS}} \phi_{i}^{\mathrm{KS}}
$$

onde $\varepsilon_{i}^{\mathrm{KS}}$ são as auto-energias de Kohn-Sham.

Ao adotar os funcionais $\Delta T[\rho]=T[\rho]-T_{s}[\rho]$ e $\Delta V[\rho]=V_{e e}[\rho]-\frac{1}{2} \int \frac{\rho(\mathbf{1}) \rho(\mathbf{2})}{r_{12}} d \mathbf{r}_{1} d \mathbf{r}_{2}$, Kohn e Sham reescreveram a equação Hohenberg e Kohn como

$$
E[\rho]=\int \rho(\mathbf{r}) v(\mathbf{r}) d \mathbf{r}+T_{s}[\rho]-\frac{1}{2} \int \frac{\rho(\mathbf{1}) \rho(\mathbf{2})}{r_{12}} d \mathbf{r}_{1} d \mathbf{r}_{2}+\Delta T[\rho]+\Delta V_{e e}[\rho]
$$

Se a energia cinética $T_{s}[\rho]$ e o potencial elétron-núcleo forem rescritos como, $T_{s}[\rho]=$ $-\frac{1}{2} \sum_{i}\left\langle\phi_{i}^{\mathrm{KS}}(\mathbf{1})\left|\nabla_{1}^{2}\right| \phi_{i}^{\mathrm{KS}}(\mathbf{1})\right\rangle$ e $v\left(\mathbf{r}_{i}\right)=-\sum_{K} \frac{Z_{K}}{r_{i K}}$, obtêm-se então

$$
E[\rho]=-Z_{K} \sum_{K} \int \frac{\rho(\mathbf{1})}{r_{i K}} d \mathbf{r}-\frac{1}{2} \sum_{i}\left\langle\phi_{i}^{\mathrm{KS}}(\mathbf{1})\left|\nabla_{1}^{2}\right| \phi_{i}^{\mathrm{KS}}(\mathbf{1})\right\rangle-\frac{1}{2} \int \frac{\rho(\mathbf{1}) \rho(\mathbf{2})}{r_{12}} d \mathbf{r}_{1} d \mathbf{r}_{2}+E_{t c}[\rho]
$$

na qual, $E_{x c}[\rho]=\Delta T[\rho]+\Delta V_{e e}[\rho]$ é chamada de energia de troca e correlação. Como o sistema fictício $s$ tem a mesma densidade do sisteam real, tem-se então $\rho=\rho_{s}=\sum_{i}\left|\phi_{i}^{\mathrm{KS}}\right|^{2}$, 
logo, expressão acima torna-se o problema de auto-vetores e auto-valores

$$
\left[-\frac{1}{2} \sum_{i} \nabla_{1}^{2}+v^{\mathrm{KS}}(\mathbf{1})\right] \phi_{i}^{\mathrm{KS}}(\mathbf{1})=\varepsilon_{i}^{\mathrm{KS}} \phi_{i}^{\mathrm{KS}}(\mathbf{1}),
$$

no qual $v^{\mathrm{KS}}[\rho]=-\sum_{K} \int \frac{Z_{K}}{r_{i K}} d \mathbf{r}-\frac{1}{2} \int \frac{\rho(\mathbf{2})}{r_{12}} d \mathbf{r}_{2}+v_{x c}(\mathbf{1})$.

Note-se que é uma estratégia útil reescrever a energia cinética sob a forma matricial. No método Kohn-Sham, admitindo uma densidade eletrônica inicial $\rho$, obtem-se primeiro o potencial $v^{K S}$. Em seguida, resolve-se o problema de auto-valores acima para obter uma nova função de onda $\phi_{i}^{\mathrm{KS}}$ e a energia $\varepsilon_{i}^{\mathrm{KS}}$. Essa nova função de onda é utilizada para gerar outra densidade eletrônica. O procedimento é repetido até a convergência da energia eletrônica.

Nos capítulos seguintes, utilizamos a teoria do funcional da densidade para investigar propriedades magnéticas nucleares, como a blindagem magnética nuclear e o acoplamento entre spins nucleares em sistemas moleculares de interesse.

\subsection{Funções base}

Em princípio, orbitais moleculares deveriam ser escritos em termos de um conjunto completo de funções base, resultando em energias Hartree-Fock iguais às obtidas pelo método variacional. Essas energias são comumente referenciadas como limite HF. Um conjunto completo de funções base não é computacionalmente favorável. Assim, apenas um número finito de funções pode ser utilizado, o que gera erros. Contudo, devido à limitações computacionais é fundamental reduzir o número de funções, mas sempre buscando minimizar os erros devido à incompleteza da base.

As funções base escolhidas são usualmente reais. Uma escolha razoável é o uso de orbitais do tipo Slater (STOs - Slater type orbitals), os quais são inspirados em funções de onda hidrogenoides. Em coordenadas esféricas, os orbitais STO são escritos [58, 62] como:

$$
\mathrm{STO}=N r^{n-1} e^{-\alpha r} \frac{\left(Y_{l}^{m *} \pm Y_{l}^{m}\right)}{2^{1 / 2}}
$$

Tratar um conjunto completo dos orbitais de Slater implica no cálculo de todas as integrais dos números quânticos $n, l$ e $m$, bem como, todos os valores do expoente positivo $\alpha$. Na prática, somente um número pequeno dessas funções pode ser usado, além do que, calcular todas as integrais de três e quatro centros, consome um tempo de computação exagerado $[54,58]$.

Em 1950, Boys [61] introduziu funções gaussianas (GTFs - gaussian type functions) na representação dos orbitais atômicos, ao invés dos tradicionais STOs. Em coordenadas cartesianas, um orbital GTF é escrito [58, 62] como: 


$$
g_{i j k}=N x^{i} y^{j} z^{k} e^{-\zeta r^{2}} .
$$

Novamente, a origem do sistema de coordenadas $(x, y, z)$ está centrada no núcleo atômico. $i, j$ e $k$ são inteiros não negativos e $\zeta$ é o expoente positivo da gaussiana. Observe que se $i+j+k=0$, temos orbitais tipo-s, com simetria esférica. Caso $i+j+k=1$, temos os chamados orbitais tipo- $p$. Se $i+j+k=2$, gera-se funções de onda tipo- $d$. Há seis tipos de gaussianas tipo- $d$. Se for preferido, seis tipos de combinações lineares dessas funções podem ser utilizadas, cinco delas tendo o comportamento angular de orbitais tipo- $d$, e uma sexta função, com simetria esférica tipo-s. É comum reescrever os orbitais GTF em coordenadas esféricas [58] como:

$$
g_{n l m}=N r^{n-1} e^{-\zeta r^{2}} \frac{\left(Y_{l}^{m *} \pm Y_{l}^{m}\right)}{2^{1 / 2}} .
$$

A representação gaussiana dos orbitais atômicos é vantajosa, pois o produto de duas gaussianas centradas em diferentes origens resulta em uma única gaussiana centrada em um ponto intermediário. Com isso, todas as integrais de dois elétrons, calculadas sobre três ou quatro núcleos atômicos, podem ser reduzidas à integrais sobre dois centros, as quais são mais fáceis de calcular.

Para reproduzir situações físicas, um número exagerado de gaussianas precisaria ser utilizado, para evitar uma expansão desse tipo, várias gaussianas são agrupadas a fim de construir as chamadas funções contraídas. Cada gaussiana contraída $\left(x_{n}\right)$ é então escrita em termos da combinação linear de gaussianas primitivas $\left(g_{i}\right)[19,58]$. Desse modo:

$$
x_{n}=\sum_{i} d_{n i} g_{i}
$$

onde os coeficientes $d_{n i}$ são constantes mantidas fixas durante todo o processo. Já os orbitais moleculares $\psi_{m}$ são escritos combinando linearmente $x_{n}$.

$$
\psi_{m}=\sum_{n} c_{n m} x_{n}
$$

Note que o uso de funções contraídas, ao invés de gaussianas primitivas, reduz brutalmente o número de coeficientes $c_{n m}$ a ser calculado auto-consistentemente.

Muitos cálculos em estrutura eletrônica começam pela escolha de funções base apropriadas ao trato da propriedade de interesse. Como exemplo, Dunning e colaboradores [63, 64, 65, 66] têm desenvolvido um conjunto de funções gaussianas contraídas, designadas cc-pVnZ ( $n=2,3,4,5$ e 6 ), desenvolvidas para serem usadas com métodos que considerem correlação eletrônica e onde propriedades que dependam dos elétrons de valência são o foco. Essas funções têm sido sistematicamente modificadas, gerando as bases aug-cc- 
pVnT-J [67, 68], desenvolvidas especialmente para o estudo de propriedades magnéticas nucleares em átomos e moléculas. A proposta das funções base aug-cc-pVnT-J, consiste em contrair mais funções gaussianas tipo-s para descrever os elétrons mais próximos ao núcleo, uma vez que o acoplamento entre spins nucleares depende fortemente dos orbitais com simetria esférica. Mesmo efeitos relativísticos, por exemplo, podem ser sistematicamente incluídos por meio de funções base como cc-pVnZ-DK [69, 70, 71, 72, 73, 74], desenvolvidas no esquema Douglas-Kroll-Hess. Há vários métodos para contrair gaussianas, uma discussão mais detalhada sobre o tema pode ser obtida nas referências [19, 58]. 


\section{Capítulo 3}

\section{Simulações clássicas Monte Carlo}

Utilizamos um tratamento sequencial Monte Carlo/Mecânica Quântica (s-MC/MQ) [75] para estudar líquidos moleculares de interesse. Inicialmente as configurações do líquido foram geradas através de simulações clássicas Monte Carlo para subsequente tratamento com mecânica quântica. Neste capítulo serão discutidos alguns detalhes relevantes do método de simulação usado.

Todas as simulações clássicas MC foram administradas partindo de uma configuração inicial construída distribuindo aleatoriamente as moléculas do líquido em uma caixa cúbica. Isso faz com que essa configuração inicial possua uma energia total muito alta. Novas estruturas são geradas de modo a obter uma cadeia de configurações com pesos estatísticos bem definidos.

As simulações foram divididas em duas etapas. Inicialmente, executando um estágio de termalização, onde o sistema é resfriado até que o equilíbrio termodinâmico seja obtido. Em uma segunda etapa, uma simulação mais longa foi administrada e muitos passos MC foram executados de modo a produzir estruturas suficientes para subsequente tratamento com mecânica quântica. Um passo MC é definido aqui quando todas as moléculas do líquido são transladadas de um comprimento $\delta \mathbf{r}$ e rotacionadas por um ângulo $\delta \theta$, compreendido entre $-15^{\circ}$ e $+15^{\circ}$ em torno de um de seus eixos. Tanto a escolha dos eixos de rotação quanto a ordem de seleção das moléculas são aleatórias.

Os efeitos de borda causados pela interação entre as moléculas e as arestas da caixa foram evitados através do método das réplicas, garantindo que todos os corpos estejam submetidos às mesmas condições. É importante perceber que se uma molécula sair da caixa original, uma de suas imagens imediatamente emergirá pela face oposta, mantendo assim, o número de partículas inalterado. Para limitar as interações, definiu-se um raio de corte, $r_{c}$, com magnitude igual a meio comprimento da caixa. Isso garante que uma molécula não possa interagir com sua própria imagem. Todos os sistemas foram simulados no ensemble NPT. Nas próximas seções serão discutidos aspectos importantes como, 
a forma de gerar as configurações para o líquido, o processo de amostragem dessas estruturas, e como considerar as interações intermoleculares.

\subsection{Cadeia de Markov (gerando configurações para um líquido)}

Em processos estocásticos deve-se construir um conjunto de configurações onde cada estrutura é gerada segundo um peso probabilístico. Uma cadeia de configurações com essas características é conhecida como cadeia de Markov [76, 77].

Para obter uma cadeia markoviana, define-se uma matriz de transição estocástica, $\pi_{i j}$, que é a probabilidade de transição entre as configurações $\Gamma_{i}$ e $\Gamma_{j}$ [77]. Desse modo:

$$
\pi_{i j} \geq 0, \sum_{i} \pi_{i j}=\mathbf{1} \forall j \text { e } \varrho_{i} \pi_{i j}=\varrho_{j} \pi_{j i} .
$$

Note-se que, a probabilidade de transição de uma configuração $\Gamma_{i}$ à $\Gamma_{j}$ é sempre não nula, a soma de todas as probabilidades de transições é igual à unidade, e os processos de transição são reversíveis.

O método Metropolis trabalha com uma matriz de transição $\pi$ dada por

$$
\begin{aligned}
& \pi_{i j}=\mathbf{1} \quad \text { se } \varrho_{j} \geq \varrho_{i} \quad \text { para } i \neq j \\
& \pi_{i j}=\frac{\varrho_{j}}{\varrho_{i}} \quad \text { se } \varrho_{j}<\varrho_{i} \quad \text { para } i \neq j \\
& \pi_{i j}=\mathbf{1}-\sum_{i \neq j} \pi_{i j} \quad \text { se } i=j
\end{aligned}
$$

É evidente essa matriz depende apenas da forma da distribuição $\varrho_{e n s}$, que por sua vez é escolhida de acordo com o ensemble simulado. No caso do ensemble NPT, têm-se que:

$$
\begin{array}{lll}
\pi_{i j}=\mathbf{1} & \text { se } U_{j} \geq U_{i} & \text { para } i \neq j \\
\pi_{i j}=e^{-\beta\left[\Delta U+p \Delta V+N \ln \left(V_{j} / V_{i}\right)\right]} & \text { se } U_{j}<U_{i} & \text { para } i \neq j \\
\pi_{i j}=\mathbf{1}-\sum_{i \neq j} \pi_{i j} & \text { se } i=j &
\end{array}
$$

O termo $N \ln \left(V_{j} / V_{i}\right)$ é resultado do re-escalonamento de todas as posições moleculares promovido pelas variações de volume. Este termo surge na simulação para evitar algumas moléculas fiquem fora da caixa, ou que existam espaços vazios quando o volume aumentar ou diminuir.

O processo markoviano para gerar configurações pode ser descrito partindo de uma 
configuração inicial $\Gamma_{i}$ e seguindo as etapas:

1- Gera-se aleatoriamente uma nova configuração $\Gamma_{j}$.

2- Calcula-se a variação de energia $\Delta U=U_{j}-U_{i}$.

3- Se $\Delta U<0$, a nova configuração $\Gamma_{j}$ é imediatamente aceita. Então retorna-se ao passo 2 gerando uma nova configuração $\Gamma_{n}$.

4- Se $\Delta U>0$, então calcula-se o peso de Boltzmann $\pi_{i j}$.

5- Paralelamente gera-se um número aleatório $\xi \in[0,1]$.

6- Se $\xi<\pi_{i j}$, aceita-se a nova configuração retornando ao passo 1 .

7- Se $\xi \geq \pi_{i j}$, a nova configuração será a anterior e retorna-se ao passo 1.

\subsection{Amostragem de Metropolis}

O valor médio de um observável está diretamente ligado a integral:

$$
\langle A\rangle_{\text {ens }}=\frac{\int_{\{\Gamma\}} A(\Gamma) \varrho_{\text {ens }} d \Gamma}{\int_{\{\Gamma\}} \varrho_{\text {ens }}(\Gamma) d \Gamma}
$$

onde $\{\Gamma\}$ delimita um espaço configuracional, $\varrho_{e n s}$ é a probabilidade de medir $A(\Gamma)$ para um dado ensemble. A integral acima envolve todo o espaço de configurações, que é infinito, além de também incluir estruturas com baixa probabilidade de ocorrerem em sistemas reais. Essas duas características tornam essa integração um problema complicado do ponto de vista computacional. Uma alternativa é obter os valores médios considerando apenas um número reduzido de configurações mais representativas. Uma dessas propostas deve-se a Metropolis e colaboradores [78].

Vamos considerar uma média para um número finito de configurações:

$$
\langle A\rangle_{e n s}=\lim _{l \rightarrow \infty} \frac{\sum_{l} A_{i} e^{-\beta U_{i}}}{\sum_{l} e^{-\beta U_{i}}} .
$$

A ideia é selecionar configurações com uma dada probabilidade $\varrho_{e n s}$, o que implica que devemos transformar a soma acima em:

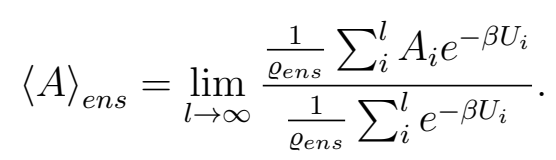

A função $\varrho_{e n s}$ pode ser qualquer uma, desde que gere uma dada distribuição. No método Monte Carlo Metropolis, escolhe-se configurações levando em conta o peso de Boltzmann. Assim, a substituição

$$
\varrho_{e n s}=e^{-\beta U}
$$


acarreta em

$$
\langle A\rangle_{e s n}=\frac{1}{l} \sum_{i=1}^{l} A_{i} .
$$

onde $l$ é o número de configurações. É claro que um erro estatístico é gerado pelo número finito de configurações. Esse erro é dado por:

$$
\sigma=\sqrt{\frac{\left\langle A^{2}\right\rangle-\langle A\rangle^{2}}{l}}
$$

onde $\left\langle\delta A^{2}\right\rangle=\left\langle A^{2}\right\rangle-\langle A\rangle^{2}$ é a flutuação do observável $A_{i}$.

Vimos que os estados são gerados com probabilidade dada pelo peso de Boltzmann. Isso conduz a duas importantes conseqüências: (1) estados que não sejam estatisticamente representativos são filtrados pela amostragem de Metropolis. Ou seja, o espaço de configurações antes infinito, reduz-se a um número limitado de configurações mais representativas e mais prováveis; (2) $\langle A\rangle$ torna-se uma média simples das grandezas $A_{i}=A\left(\Gamma_{i}\right)$, uma vez que o peso de Boltzmann já está considerado.

\subsection{Eficiência e correlação estatística}

Vimos como construir uma cadeia de configurações. Em simulações MC, esse número é tipicamente da ordem de $\sim 10^{9}$ estruturas. Contudo, não há necessidade de tratar todas essas configurações com mecânica quântica. Vimos que a amostragem de Metropolis garante a possibilidade de obter o valor médio de um observável considerando apenas uma quantidade reduzida de estruturas, desde que sejam as mais representativas. Nesta seção discutiremos como selecionar configurações com essas características.

Uma simulação é eficiente se há pouca correlação estatística entre suas configurações. Podemos selecionar configurações suficientemente descorrelacionadas usando funções de auto-correlação. No caso de simulações Monte Carlo, usa-se a função de auto-correlação da energia $C(i)$ :

$$
C(i)=\frac{\left\langle\delta E_{n} \delta E_{n+i}\right\rangle}{\left\langle\delta E^{2}\right\rangle}=\frac{\sum_{n}\left(E_{n}-\langle E\rangle\right)\left(E_{n+i}-\langle E\rangle\right)}{\sum_{n}\left(E_{n}-\langle E\rangle\right)^{2}},
$$

onde $i$ é o número de passos MC, $E_{n}$ é a energia da $n$-ésima configuração, $E_{n+i}$ é a energia da configuração gerada $i$ passos MC depois. Em processos markovianos, as funções de auto-correlação são dadas por funções exponenciais do tipo [75]:

$$
C(i)=\sum_{i}^{n} c_{i} e^{-i / \tau_{i}}
$$


onde $\tau_{i}$ e $c_{i}$ são constantes. Para líquidos em geral, $C(i)$ é dada por um decaimento exponencial de segunda ordem:

$$
C(i)=c_{1} e^{-i / \tau_{1}}+c_{2} e^{-i / \tau_{2}}
$$

Entende-se por passo de correlação $\tau$ o intervalo obtido integrando a função $C(i)$ partindo do ponto onde as configurações estão totalmente correlacionadas $(i=0)$ até o ponto onde a correlação é nula $(i=\infty)$. Desse modo:

$$
\tau=\int_{0}^{\infty} C(i) d i
$$

Relacionando as equações (3.17) e (3.18), obtemos o intervalo $\tau$ em função das constantes $\tau_{i}$ e $c_{i}$ :

$$
\tau=c_{1} \tau_{1}+c_{2} \tau_{2}
$$

Em geral, configurações separadas por intervalos $i>2 \tau$ estão estatisticamente correlacionadas em torno de $15 \%$.

\subsection{Função de distribuição radial}

A estrutura de um líquido é melhor estudada por meio do conceito de função de distribuição radial esférica (FDR). Normalmente FDRs são usadas para definir a formação ligações de hidrogênio e as camadas de solvatação em torno do soluto, fornecendo informações sobre a coordenação do solvente. É possível escrever uma FDR em termos da integração da função de distribuição configuracional do ensemble.

$$
G\left(r_{1}, r_{2}\right)=\frac{N(N-1)}{\rho^{2} Z} \int \varrho_{e n s} d^{3} \mathbf{r}_{3} d^{3} \mathbf{r}_{4} \ldots d^{3} \mathbf{r}_{n}
$$

onde $\varrho_{e n s}$ é a distribuição de Boltzmann em umda do ensemble. Integrando uma FDR em uma calota esférica centrada em torno de uma molécula previamente escolhida, determinase o número $N_{s}(r)$ de moléculas inseridas na calota:

$$
N_{s}(r)=\frac{1}{V} \int_{V} G(r) r^{2} d r d \Omega
$$

sendo $d \Omega$ o elemento de ângulo sólido. Define-se o número de moléculas que resultam da integração do primeiro pico como a primeira camada de solvatação. A quantidade de moléculas presentes nas demais camadas de solvatação, são dadas pela integração dos demais picos. 


\subsection{Potencial interatômico}

O Hamiltoniano clássico é construído em termos das interações intramoleculares e intermoleculares:

$$
H_{M M}=H_{\text {intra }}+H_{\text {inter }}
$$

A parte intramolecular é dada por:

$$
H_{M M}=H_{\text {str }}+H_{\text {bend }}+H_{\text {tor }}+H_{v d W}+H_{\text {ele }}+H_{\text {cross }}
$$

sendo que: (1) $H_{\text {str }}$ descreve o estiramento das ligações; (2) $H_{\text {bend }}$ modela a variação nos ângulos de ligação; (3) $H_{t o r}$ descreve os ângulos de torção; (4) $H_{v d W}$ modela as interações de van der Waals; (5) $H_{\text {ele }}$ trata das interações eletrostáticas; (6) embora não seja muito utilizado, $H_{\text {croos }}$ possibilita descrever acoplamentos entre diversos termos. Contudo, modelos rígidos de moléculas foram utilizados em todas as simulações Monte Carlo realizadas neste trabalho. Isso significa que somente as interações intermoleculares foram consideradas.

Neste caso, o hamiltoniano é dado como

$$
H_{\text {inter }}=\sum_{i}^{\mathrm{em}} \sum_{j}^{a \mathrm{em} b} H\left(r_{i j}\right)
$$

sendo $i$ e $j$ os sítios nas moléculas $a$ e $b$, respectivamente.

Na descrição Monte Carlo de sistemas moleculares solvatados, para moléculas mantidas rígidas, costuma-se adicionar os potencias de Lennard-Jones (LJ) e Coulomb:

$$
H_{i j}=\sum_{i}^{\text {em } a \text { em } b} \sum_{j}^{b}\left\{4 \epsilon_{i j}\left[\left(\frac{\sigma_{i j}}{r_{i j}}\right)^{12}-\left(\frac{\sigma_{i j}}{r_{i j}}\right)^{6}\right]+\frac{e^{2}}{4 \pi \epsilon_{0}} \frac{q_{i} q_{j}}{r_{i j}}\right\}
$$

onde $\sigma_{i j}=\sqrt{\sigma_{i} \sigma_{j}}, \epsilon_{i j}=\sqrt{\epsilon_{i} \epsilon_{j}}, e^{2} / 4 \pi \epsilon_{0}=331.9684 \AA \mathrm{kcal} / \mathrm{mol}$ e $q_{i}$ representa carga em cada sítio. As grandezas $\sigma_{i}$ e $\epsilon_{i}$ correspondem à distância e às energias entre os sítios quando o potencial interatômico tem valores nulo e mínimo, respectivamente. Esses parâmetros podem ser determinados empiricamente ou por métodos computacionais. Existem alguns conjuntos de parâmetros que obtiveram notoriedade, como é o caso do campo de forças OPLS (Optimized Parameters for Liquid Simulation) [79]. Campos de força normalmente definem cada átomo como um sítio molecular. Contudo, dependendo da propriedade de interesse, pode-se usar potenciais que definam maior ou menor número de sítios que a quantidade de átomos na molécula. O campo de forças TIP5P desenvolvido por Jorgensen por exemplo, descreve a molécula de água através de cinco sítios. Já o potencial desenvolvido por van Gunsteren, descreve o dimetil-sulfóxido $\left[\left(\mathrm{CH}_{3}\right)_{2} \mathrm{SO}\right]$ 
através de quatro sítios.

No potencial de LJ, o termo atrativo responsável pelas interações dipolares tem origem física nas forças de van der Waals. O primeiro termo é repulsivo, sendo predominante para pequenas distâncias. Embora sua inclusão tenha uma justificativa física, sua dependência em $r^{-12}$ é adotada apenas por melhor ajustar-se ao termo de van der Waals na descrição das interações moleculares. A adição do potencial de Coulomb se justifica, para descrever as interações eletrostáticas e momentos de multipolos. 


\section{Capítulo 4}

\section{Ressonância magnética nuclear}

Sabe-se que alguns núcleos possuem momento magnético. Essa hipótese foi conjecturada pela primeira vez por Pauli [80] para explicar detalhes da estrutura fina no espectro ótico de alguns elementos. Ressonânia magnética nuclear (RMN) é um fenômeno físico, no qual núcleos atômicos, sob ação de um campo magnético absorvem e emitem energia. Se os núcleos não possuem momento magnético, nenhum espectro RMN pode ser observado. Esses efeitos foram reportados pela primeira vez por Rabi [1] e, mais tarde, Bloch, Hansen e Packard [2], bem como Purcell, Torrey e Pound [3] desenvolveram técnicas para o estudo em sólidos e líquidos.

O espectro RMN se utiliza de núcleos magnéticos para sondar e obter informações com respeito à estrutura da matéria. Campos magnéticos locais, próximos a núcleos magnéticos, dependem de uma série de fatores, dentre eles, a polarização da amostra, momentos magnéticos (nucleares e eletrônicos) das moléculas vizinhas e, efeitos intramoleculares devido a elétrons e outros núcleos. Essa forma de espectroscopia é potencialmente importante em problemas envolvendo a estrutura molecular e o ambiente químico, tendo aplicações em diversos ramos da química. Abordaremos agora alguns fatores fundamentais em RMN.

\subsection{Momento magnético nuclear}

Com respeito a suas propriedades magnéticas, núcleos são descritos em termos de seus momentos angulares e spins. De acordo com a mecânica quântica, a componente máxima do momento angular de qualquer sistema é um múltiplo da constante de Plank modificada, $\hbar=h / 2 \pi$. Se escrevermos essa componente simplemente como $I$, então, $I$ é também o número quântico de spin do sistema. Sabe-se que um núcleo terá $2 I+1$ diferentes estados e, na ausência de campos magnéticos, esses estados terão a mesma energia.

Todos os dados sobre propriedades magnéticas concordam que o momento magnético 
Tabela 4.1: Classificação do spin nuclear segundo o números de massa e número atômico de um átomo:

\begin{tabular}{lll}
\hline \hline número de massa $(\boldsymbol{A})$ & número atômico $(\boldsymbol{Z})$ & spin nuclear $(\boldsymbol{I})$ \\
\hline ímpar & par ou ímpar & $\frac{1}{2}, \frac{3}{2}, \frac{5}{2} \ldots$ \\
par & par & 0 \\
par & ímpar & $1,2,3 \ldots$ \\
\hline \hline
\end{tabular}

$\mu$ é nulo, quando $I=0$ e, para situações em que $I$ não se anula, o momento magnético é sempre paralelo ao momento angular. Pode-se então definir a componente máxima do momento magnético, como

$$
\boldsymbol{\mu}=\gamma \hbar \mathbf{I}
$$

onde $\gamma$ é uma constante característica de cada núcleo, conhecida como constante giromagnética nuclear, ou apenas, raio giromagnético.

Com respeito aos spins nucleares, há certas características regulares que podem ser expressas em termos do número atômico $Z$ e do número de massa $A$ dos núcleos. (1) Se o número de massa $A$ é ímpar, o spin nuclear $I$ não é inteiro. (2) Se o número de massa $A$ e carga atômica $Z$ são ambos pares, o spin é nulo. (3) Se o número de massa $A$ é ímpar, mas o número atômico $Z$ é par, então, o spin é inteiro. A tabela 4.1 resume essas características.

Núcleos com spin $I=0$ não possuem espectro RMN. Note-se que muitos núcleos comuns, como o ${ }^{12} \mathrm{C}$ e ${ }^{16} \mathrm{O}$ integram essa categoria. Isso significa que um número muito grande de moléculas contendo $\mathrm{H}, \mathrm{C}$ e $\mathrm{O}$, proporcionam, livre de complicações, apenas o espectro magnético ressonante do próton, uma vez que em natura, há baixas concentrações dos isótopos ${ }^{13} \mathrm{C} \mathrm{e}{ }^{17} \mathrm{O}$.

\subsection{Níveis de energia em um campo magnético}

Quando núcleos magnéticos são embebidos em um campo magnético uniforme $B_{0}$, arbitrariamente orientado na direção $z$, suas energias são dadas por

$$
-\mu_{z} B_{0}
$$

onde $\mu_{z}$ é a componente $z$ do momento magnético nuclear. Um núcleo, com spin nuclear $I$, e momento $\mu_{z}$ com $2 I+1$ componentes, os níveis de energia dos vários estados serão 


\section{Energia}

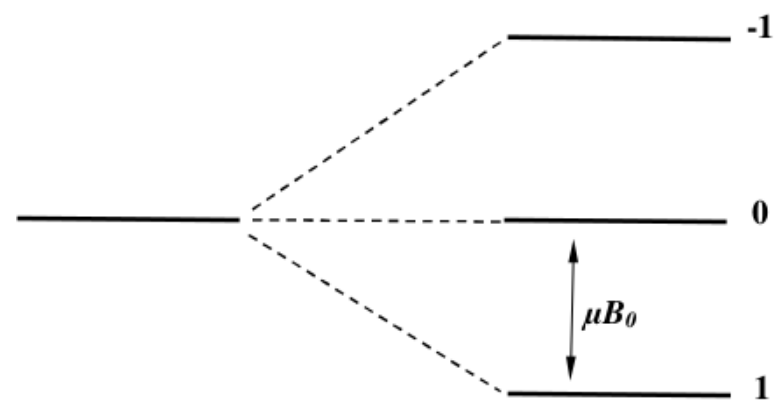

$+\mu B_{0}$

0

$-\mu \boldsymbol{B}_{0}$

Figura 4.1: Efeito Zeeman para um núcleo de momento angular $I=1$ em um campo magnético.

igualmente espaçados e dados por

$$
-\mu_{z} B_{0},-\frac{I-1}{I} \mu B_{0}, \ldots,-\frac{I-1}{I} \mu B_{0},-\frac{I+1}{I}, \mu B_{0} .
$$

Essa separação entre os níveis de energia é conhecida como efeito Zeeman nuclear, com clara analogia ao seu análogo eletrônico. A figura 4.1 ilustra efeito Zeeman nuclear para um núcleo de spin 1, que por consequência, tem apenas três níveis de energia.

A ideia básica em RMN é induzir transições entre esses níveis pela absorção ou emissão de energia. Uma transição entre dois níveis vizinhos é dada pela condição de frequência de Bohr

$$
h \nu=\frac{\mu B_{0}}{I}
$$

ou, em termos da constante giromagnética

$$
\nu=\frac{\gamma B_{0}}{2 \pi}
$$

\subsection{Blindagem magnética nuclear devida aos elétrons}

Um importante efeito do meio tem origem na nuvem eletrônica ao redor dos núcleos. Qualquer núcleo, submetido à ação de um campo magnético, adquire um momento diamagnético em virtude do movimento orbital eletrônico induzido. Os elétrons se comportam como correntes efetivas e, portanto, produzem um campo magnético secundário, $B_{\text {local }}$, que atua sobre todos os núcleos presentes. Uma vez que as correntes induzidas são proporcionais ao campo magnético aplicado, $B_{0}$, a magnitude do campo secundário também é proporcional ao campo aplicado, sendo dada por 


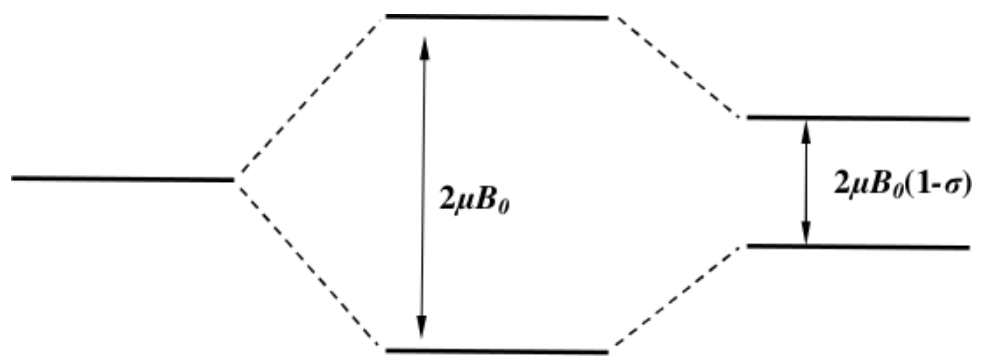

Figura 4.2: Eleito da blindagem magnética nuclear sobre os níveis de energia Zeeman de um núcleo com momento angular $I=\frac{1}{2}$.

$$
B_{\text {local }}=B_{0}(1-\sigma)
$$

onde $\sigma$ é uma constante adimensional, independente do campo aplicado, $B_{0}$, mas dependente do ambiente químico (eletrônico). A constante $\sigma$ é chamada de blindagem magnética nuclear. Sua magnitide varia aproximadamente de $10^{-5}$, para prótons, até $10^{-2}$, para elementos mais pesados.

A expressão geral para a blindagem magnética sobre um dado núcleo foi primeiro deduzida por Ramsey, em 1950 [81]. Propriedades magnéticas, em geral, são dependentes da orientação molecular em relação ao campo magnético aplicado. Desse modo, a blindagem é escrita como um tensor de segunda orden, com nove componentes distintas especificando sua orientação com relação à origem. Temos então

$$
\sigma=\left(\begin{array}{lll}
\sigma_{11} & \sigma_{12} & \sigma_{13} \\
\sigma_{21} & \sigma_{22} & \sigma_{23} \\
\sigma_{31} & \sigma_{32} & \sigma_{33}
\end{array}\right)
$$

Em experimentos RMN convencionais, sobre tudo em líquidos, é complicado determinar todas as componentes desse tensor, assim, argumentos de simetria são usados e somente a contribuição isotrópica $\sigma_{\text {iso }}$ é utilizada. Essa componente é dada pelo traço da matriz acima, sendo escrita como

$$
\sigma_{\text {iso }}=\frac{1}{3}\left(\sigma_{11}+\sigma_{22}+\sigma_{33}\right)
$$

O efeito da blindagem magnética consiste em aproximar os níveis de energia Zeeman, como mostrado na figura 4.2. Em consequência, a energia quântica requerida para promover transições entre dois níveis consecutivos é menor, portanto, as ressonâcias ocorrem também à frequências menores, dadas por

$$
\nu=\frac{\gamma H_{0}(1-\sigma)}{2 \pi}
$$




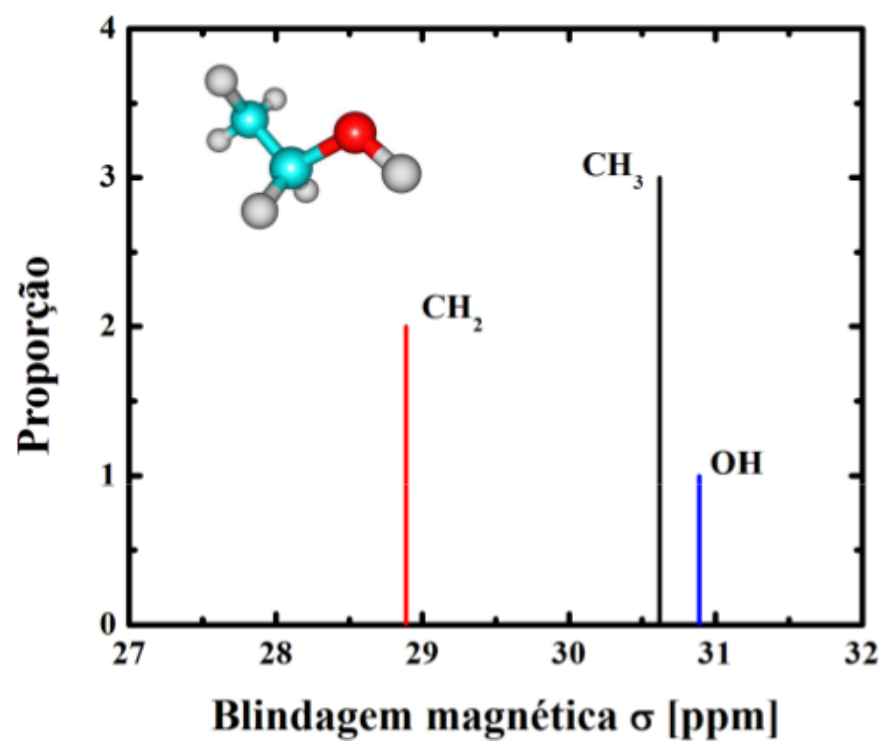

Figura 4.3: Espectro ressonante dos prótons calculado [B3LYP/6-311++G(d,p)] no álcool etílico.

Se analizarmos o espectro RMN de um dado elemento em vários ambientes químicos distintos, seja em diferentes moléculas ou em diferentes posições na mesma molécula, observaremos conjuntos blindagens magnéticas distintas. Isso significa que em espécies experimentando diferentes ambientes químicos, a ressonância ocorre em diferentes partes do espectro. Com exemplo, considere o álcool etílico, $\mathrm{CH}_{3} \mathrm{CH}_{2} \mathrm{OH}$, onde há três tipos de prótons. A figura 4.3 mostra as blindagens magnética calculadas para esse composto em nível de aproximação B3LYP $/ 6-311++\mathrm{G}(d, p)$. No espectro são observados três sinais bem espassados, correspondendo aos três tipos de prótons encontrados na molécula. Note-se que os picos têm proporções 2:3:1, proporcionando imediata identificação. Uma descrição mais detalhada sobre a blindagem magnética e outras constantes magnéticas nucleares de interesse pode ser encontrada na referência [82].

\subsection{Dependência de propriedades magnéticas com re- lação à origem}

Há fatores que dificultam o cálculo de propriedades magnéticas. Dentre eles, a dependência de operadores importantes à propriedades magnéticas, como o momento angular, com relação à origem, implicando em resultados igualmente dependentes da escolha de calibre. Uma função de onda exata, evidentemente, eliminaria esses problemas, mas devido às limitações numéricas, um conjunto finito de funções base precisa ser utilizado.

Uma implementação recente, que elimina a dependência das propriedades com relação ao calibre, torna as funções de onda explicitamente dependentes do campo magnético. $\mathrm{O}$ 
método inclui um fator de fase complexo, o qual referencia a origem das funções base, usualmente, os núcleos atômicos. Nessa aproximação, orbitais são escritos como

$$
X_{\mathrm{A}}\left(\mathbf{r}-\mathbf{R}_{\mathrm{A}}\right)=e^{-\frac{i \mathbf{A}_{\mathrm{A}} \cdot \mathbf{r}}{c}} \chi_{\mathrm{A}}\left(\mathbf{r}-\mathbf{R}_{\mathrm{A}}\right)
$$

onde

$$
\chi_{\mathrm{A}}\left(\mathbf{r}-\mathbf{R}_{\mathrm{A}}\right)=N e^{-\alpha\left(\mathbf{r}-\mathbf{R}_{\mathrm{A}}\right)}
$$

e

$$
\mathbf{A}_{\mathrm{A}}=\frac{1}{2} \mathbf{B} \times\left(\mathbf{R}_{\mathrm{A}}-\mathbf{R}_{\mathrm{G}}\right)
$$

Esses orbitais são mais conhecidos como Orbitais atômicos de London (LAO - London atomic orbitais) [83] ou apenas GIAO (Gauge including atômic orbitals) [84]. Com efeito, note-se que as matrizes que evolvem o potencial vetor $\mathbf{A}_{\mathrm{A}}$, somente contém a diferença entre as posições de dois núcleos, o que remove a dependência relativa à origem. Com respeito à matrizes de conbrimento $\left\langle\chi_{\mathrm{A}} \mid \chi_{\mathrm{B}}\right\rangle$ e energia potencial $\left\langle\chi_{\mathrm{A}}|\mathbf{V}| \chi_{\mathrm{B}}\right\rangle$, é simples notar que seus elementos são independentes da origem. À saber

$$
\left\langle\chi_{\mathrm{A}} \mid \chi_{\mathrm{B}}\right\rangle=\left\langle\chi_{\mathrm{A}}\left|e^{\frac{i\left(\mathbf{A}_{\mathrm{A}}-\mathbf{A}_{\mathrm{B}}\right) \cdot \mathbf{r}}{c}}\right| \chi_{\mathrm{B}}\right\rangle
$$

$\mathrm{e}$

$$
\left\langle\chi_{\mathrm{A}}|\mathbf{V}| \chi_{\mathrm{B}}\right\rangle=\left\langle\chi_{\mathrm{A}}\left|e^{\frac{i\left(\mathbf{A}_{\mathrm{A}}-\mathbf{A}_{\mathrm{B}}\right) \cdot \mathbf{r}}{c}} \mathbf{V}\right| \chi_{\mathrm{B}}\right\rangle
$$

Quanto à energia cinética $\mathbf{K}$, embora sua expressão seja um pouco mais complicada, é possível mostrar que

$$
\left\langle\chi_{\mathrm{A}}|\mathbf{K}| \chi_{\mathrm{B}}\right\rangle=\left\langle\chi_{\mathrm{A}}\left|e^{\frac{i\left(\mathbf{A}_{\mathrm{A}}-\mathbf{A}_{\mathrm{B}}\right) \cdot \mathbf{r}}{c}}(\mathbf{p}+\mathbf{A})^{2}\right| \chi_{\mathrm{B}}\right\rangle
$$

Note-se que na presença de campos magnético, o momento linear p é substituído por $\mathbf{p}+\mathbf{A}$. Está claro que o uso dos orbitais GIAO torna todos os elementos de matriz e, propriedades correlatas, independentes da escolha da origem.

\subsection{Acoplamento entre spins nucleares}

O acoplamento indireto entre spins nucleares é um dos parâmetros fundamentais que caracterizam o espectro RMN de alta resolução. Essas constantes podem ser calculadas como propriedades moleculares de segunda ordem utilizando teoria de perturbações inde- 
pendente do tempo, onde a perturbação é o campo magnético aplicado [85]. Na prática, o cálculo do acoplamento entre spin nucleares é uma tarefa mais complicada do que outras propriedades moleculares de segunda ordem, como polarizabilidades, magnetizabilidades e blindagens magnéticas nucleares [86]. Dentre as dificuldades, destacam-se primeiro, em nível não relativístico, as contribuições de vários mecanismos distintos [85]. Entre eles, o mecanismo de spin-orbital diamagnético (DSO) e paramagnético (PSO), bem como os termos de contato de Fermi (FC) e o acoplamento spin-dipolo (SD). Todos esses mecanismos têm contribuições importantes, não podendo ser negligenciados. Segundo, o acoplamento entre spins nucleares envolve excitações triplas, as quais requerem níveis de cálculo mais apurados e uma descrição mais sofisticada do sistema eletrônico. Por fim, o termo de contato de Fermi, frequentemente dominante, depende criticamente da densidade eletrônica próxima ao núcleo, com isso, a convergência do conjunto de base é lenta e bases muito maiores precisam ser empregadas para garantir resultados consistentes.

Como consequência, o cálculo do acoplamento indireto entre spins nucleares é uma propriedade que requer um tempo de computação muito maior, quando comparado à propriedades como a blindagem magnética nuclear, contudo, ambas precisam ser obtidas para garantir uma boa descrição do espectro MNR.

Nesta seção, discutiremos brevemente a expressão obtida por Ramsey [85] para o acoplamento indireto entre spins nucleares. Os acoplamentos são obtidos como derivadas da energia eletrônica, que por sua vez é calculada na presença de um campo magnético aplicado. Inicialmente, considere o momento magnético nuclear

$$
\boldsymbol{\mu}=\gamma \hbar \mathbf{I}
$$

Os acoplamentos normal e reduzido estão relacionados como

$$
\mathbf{J}_{K L}=h \frac{\gamma_{K}}{2 \pi} \frac{\gamma_{L}}{2 \pi} \mathbf{K}_{K L}=h \frac{\gamma_{K}}{2 \pi} \frac{\gamma_{L}}{2 \pi} \frac{d^{2} E}{d \boldsymbol{\mu}_{K} d \boldsymbol{\mu}_{L}}
$$

onde os índices correm sobre todos o núcleos. Na aproximação de Born-Oppenheimer, o hamiltoniano não relativístivo, na presença de campos magnéticos aplicados e em unidades atômicas, é dado como

$$
H=\frac{1}{2} \sum_{i}\left[\mathbf{p}_{i}+\mathbf{A}\left(\mathbf{r}_{i}\right)\right] \cdot\left[\mathbf{p}_{i}+\mathbf{A}\left(\mathbf{r}_{i}\right)\right]+\sum_{i} \mathbf{s}_{i} \cdot \mathbf{B}\left(\mathbf{r}_{1}\right)+V_{e n}+V_{e e}+V_{n n}
$$

onde $\mathbf{p}_{i}$ e $\mathbf{s}_{i}$ são, respectivamente, o momento conjugado do elétron e seu spin. O vetor potencial magnético $\mathbf{A}\left(\mathbf{r}_{i}\right)$ e o campo magnético $\mathbf{B}\left(\mathbf{r}_{i}\right)=\nabla \times \mathbf{A}\left(\mathbf{r}_{i}\right)$ são escritos como 


$$
\begin{gathered}
\mathbf{A}\left(\mathbf{r}_{i}\right)=\alpha^{2} \sum_{K} \frac{\boldsymbol{\mu}_{K} \times \mathbf{r}_{i K}}{r_{i K}^{3}} \\
\mathbf{B}\left(\mathbf{r}_{i}\right)=\frac{8 \pi \alpha^{2}}{3} \sum_{K} \delta\left(\mathbf{r}_{i K}\right) \boldsymbol{\mu}_{K}+\alpha^{2} \sum_{K} \frac{3 \mathbf{r}_{i K} \mathbf{r}_{i K}^{T}-r_{i K}^{2} \mathbf{I}_{3}}{r_{i K}^{5}} \boldsymbol{\mu}_{K},
\end{gathered}
$$

nos quais $\alpha$ é a constante de estrutura fina e $\mathbf{r}_{i K}$ é a posição do elétron $i$ em relação ao núcleo $K$. Ao inserir o vetor potencial e o campo magnético no hamiltoniano acima, temos, após um rápido rearranjo

$$
H=\frac{1}{2} \sum_{i} p_{i}^{2}+V_{e n}+V_{e e}+V_{n n}+\sum_{K L} \boldsymbol{\mu}_{K}^{T}\left(\mathbf{h}_{K}^{\mathrm{PSO}}+\mathbf{h}_{K}^{\mathrm{DSO}}+\mathbf{h}_{K}^{\mathrm{FC}}+\mathbf{h}_{K}^{\mathrm{SD}}\right) \boldsymbol{\mu}_{L}
$$

no qual introduzimos os termos de spin-orbital paramagnético (PSO) e diamagnético (DSO)

$$
\begin{gathered}
\mathbf{h}_{K}^{\mathrm{DSO}}=\frac{\alpha^{4}}{2} \sum_{i} \frac{\left(\mathbf{r}_{i K}^{T} \mathbf{r}_{i L}\right) \mathbf{I}_{3}-\mathbf{r}_{i K} \mathbf{r}_{i L}^{T}}{r_{i K}^{3} r^{3}{ }_{i L}} \\
\mathbf{h}_{K}^{\mathrm{PSO}}=\alpha^{2} \sum_{i} \frac{\mathbf{r}_{i K} \times \mathbf{p}_{i}}{r^{3}{ }_{i K}}
\end{gathered}
$$

bem como os termos de contato de Fermi (FC) e o acoplamento spin-dipolo (SD).

$$
\begin{gathered}
\mathbf{h}_{K}^{\mathrm{FC}}=\frac{8 \pi \alpha^{3}}{3} \sum_{i} \delta\left(\mathbf{r}_{i K}\right) \mathbf{s}_{i} \\
\mathbf{h}_{K}^{\mathrm{SD}}=\frac{\alpha^{4}}{2} \sum_{i} \frac{3\left(\mathbf{s}_{i}^{T} \mathbf{r}_{i L}\right) \mathbf{r}_{i K}-r_{i K} \mathbf{s}_{i}}{r_{i K}^{5}} .
\end{gathered}
$$

No formalismo tradicional descrito por Ramsey [85], as expressões acima conduzem à constante reduzida de acoplamento

$$
\begin{gathered}
\mathbf{K}_{K L}=\left\langle 0\left|\mathbf{h}^{\mathrm{DSO}}\right| 0\right\rangle+2 \sum_{s>0} \frac{\left\langle 0\left|\mathbf{h}_{K}^{\mathrm{PSO}}\right| s\right\rangle\left\langle s\left|\mathbf{h}_{L}^{\mathrm{PSO}}\right| 0\right\rangle^{T}}{E_{0}-E_{s}} \\
\quad+2 \sum_{t} \frac{\left\langle 0\left|\mathbf{h}_{K}^{\mathrm{FC}}+\mathbf{h}_{K}^{\mathrm{SD}}\right| t\right\rangle\left\langle t\left|\mathbf{h}_{K}^{\mathrm{FC}}+\mathbf{h}_{K}^{\mathrm{SD}}\right| 0\right\rangle^{T}}{E_{0}-E_{t}}
\end{gathered}
$$

para a qual, a primeira soma cobre todas as excitações singleto diferentes do estado de referência e, a segunda somatória considera todos os estados tripleto. No estudo do acoplamento indireto entre spins nucleares que realizaremos a seguir, as contribuições 
desses termos serão obtidas separadamente e analisadas. 
Parte II

Aplicações 


\section{Capítulo 5}

\section{Uracila e 5-fluorouracila hidratadas}

\subsection{Introdução}

Uracila e 5-fluorouracila (figura 5.1) são importantes compostos biológicos. A uracila é usualmente encontrado no RNA ligado à adenina. Responsável por inúmeras reações reguladoras no organismo, ainda pode ser usado como medicamento anticancerígeno quando o $\mathrm{H}_{9}$ é substituído pelo flúor (veja esquema 5.1), produzindo a 5-fluoruracila. Sua funcionalidade e potencialidades têm sido exploradas em uma extensa gama de trabalhos experimentais versando sobre transferência de prótons intra e intermolecular [87, 88, 89], influência do meio sobre suas transições eletrônicas [44, 90] e desenvolvimento de fárma$\cos$ [91, 92]. Ao longo dos anos, ressonância magnética nuclear (RMN) envolvendo os elementos ${ }^{1} \mathrm{H},{ }^{13} \mathrm{C}$ e ${ }^{15} \mathrm{~N}$ têm sido uma importante ferramento no estudo de compostos de interesse biológico, como a uracila e seus derivados [93, 94, 95, 96]. Contudo, são escassos os trabalhos com respeito ao isótopo ${ }^{17} \mathrm{O}[97,98,99]$ para a molécula solvatada e totalmente nulas as observações com respeito ao gás. Estima-se que o solvente possa gerar um desvio gas-líquido dramático de até 80 ppm [100].

Embora o isótopo ${ }^{17} \mathrm{O}$ seja um elemento abundante em sistemas biológicos, é reduzido o número de trabalhos que utilizam seu espectro RMN para investigar a estrutura de biomoléculas. Sua abundância natural $(0,037 \%)$ e a dificuldade de tratar núcleos com momento quadrupolar são relatadas como as maiores dificuldades experimentais [97]. As interações quadrupolares causam eficiente relaxação do sinal RMN em sólidos, com efeitos ainda mais dramáticos sobre líquidos. Neste cenário, a modelagem molecular desempenha papel fundamental, uma vez que os avanços computacionais ocorridos nas últimas décadas têm facilitado abordagens teóricas. Em particular, grande atenção têm se voltado sobre líquidos.

Sobre ressonância magnética nuclear em ambiente líquido, não se sabe precisamente quais interações são mais relevantes e como incluí-las. Apenas recentemente Fonseca e co- 
laboradores [36] mostraram que a blindagem magnética sobre o ${ }^{13} \mathrm{C}$ em acetona hidratada é muito bem descrita garantindo os efeitos da polarização sobre o soluto e as interações eletrostáticas provenientes das moléculas do solvente mais afastadas. A pouca importância da inclusão explícita do solvente é uma enorme surpresa já que constantes RMN são propriedades reconhecidamente locais.
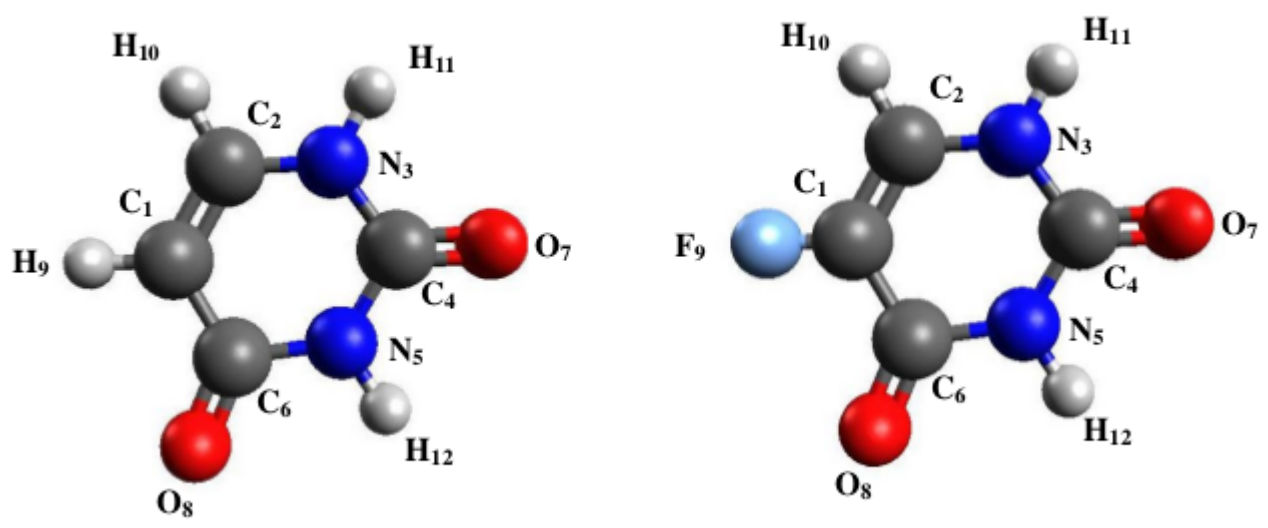

Figura 5.1: Uracila à esquerda. A 5-fluorouracila (à direita) é obtida partindo da uracila substituindo $\mathrm{H}_{9}$ por $\mathrm{F}$.

Utilizamos aproximações de química quântica para entender o papel do meio sobre constantes magnéticas nucleares em uracila solvatada. Devido à escassez de resultados e a sensibilidade do isótopo ${ }^{17} \mathrm{O}$ com relação ao meio, manteremos o foco sobre a dependência da blindagem magnética $\sigma\left({ }^{17} \mathrm{O}\right)$ com relação aos efeitos da polarização do soluto e de sua participação em interações de ligações de hidrogênio. São considerados aqui vários modelos contínuos e discretos de solventes a fim de entender e modelar essas interações.

\subsection{Detalhes computacionais}

Utilizamos a metodologia sequencial QM/MC (s-QM/MC) para gerar as estruturas do líquido para subsequente tratamento com mecânica quântica. Todas as simulações MC foram executadas utilizando o programa DICE [101] e, administradas no ensemble NPT à $95^{\circ} \mathrm{C}$ e $1 \mathrm{~atm}$, com uma molécula de referência (uracila ou 5-fluorouracila) diluída em 700 moléculas de água. As interações soluto-solvente foram mediadas pelos potenciais de Lennard-Jones (LJ) e Coulomb. O solvente foi totalmente descrito pelo potencial TIP3P [102]. Já os parâmetros LJ do soluto foram extraídos do campo de forças OPLS [79], mas suas cargas atômicas foram obtidas através de um procedimento iterativo [47, 48]. Neste procedimento, configurações MC estatisticamente descorrelacionadas são usadas 
para gerar uma configuração eletrostática média do solvente (ASEC - average solvent electrostatic configuration). Nessa configuração média o soluto é embebido no campo eletrostático proveniente das moléculas do solvente descritas apenas pelas cargas pontuais do potencial TIP3P. Essa configuração média é usada para obter as novas cargas do soluto que são novamente implementadas em uma nova simulação, na qual uma nova configuração ASEC é gerada. O processo é repetido iterativamente até a convergência de alguma propriedade molecular de interesse, no caso, o momento de dipolo. Note que a cada passo iterativo é permitido relaxar a distribuição de cargas da molécula de referência na presença do solvente. Isso garante que os efeitos da polarização do soluto devido ao solvente sejam sistematicamente incluídos. As cargas atômicas foram obtidas em nível de cálculo MP2/aug-cc-pVTZ, dentro do esquema CHELPG (Charges from Electrostatic Potentials using a Grid based method) [103], o qual é um método desenvolvido por Breneman e Wiberg para calcular as cargas atômicas, as quais são ajustadas para reproduzir o potencial eletrostático molecular em um número de pontos ao redor da molécula.

Reduzir o custo computacional sem comprometer a qualidade dos resultados é um aspecto fundamental em sistemas moleculares de grande porte. Isto implica na escolha de modelos quânticos apropriados, funções base e, principalmente, os efeitos que devem se considerados. A teoria do funcional da densidade (DFT) tem proporcionado uma descrição consistente de propriedades magnéticas em geral, com a vantagem se ser menos cara, em relação a outras aproximações mais sofisticadas. Na descrição de líquidos moleculares, onde muitos corpos devem ser considerados explicitamente, essa característica é fundamental. Hoje é possível tirar vantagem de funcionais construídos especificamente para o estudo de propriedades magnéticas nucleares em sólidos e líquidos moleculares [104]. Recentemente as performances de diferentes funcionais foram avaliadas na descrição das constantes magnéticas nucleares em metanol [105], onde a melhor correlação entre teoria e experimento foi obtida com os funcionais B3LYP [106] e PBE0 [107]. Observações similares também foram reportadas com relação ao acoplamento indireto entre spins nucleares [108]. Adicionalmente, há recentes estudos que destacam o sucesso do funcional híbrido mPW1PW91 [109, 110] no trato de propriedades estruturais e constantes magnéticas nucleares [111, 112, 113]. Com relação à contribuições relativísticas em propriedades magnéticas nucleares, Oziminski e colaboradores [113] discutiram que esses efeitos são fundamentais apenas na descrição elementos pesados, como o ${ }^{195 \mathrm{~m}} \mathrm{Pt}$.

Nesta tese discutimos os efeitos do solvente sobre a blindagem magnética nuclear $\sigma\left({ }^{17} \mathrm{O}\right)$ em uracila e 5 -fluorouracila solvatadas. Toda a discussão é feita em termos da DFT na forma dos funcionais B3LYP e mPW1PW91. Também foram utilizadas as funções base aug-pcS- $n$ [114] apropriadas para o estudo de propriedades magnéticas nucleares e desenvolvidas para serem usadas junto à DFT. Todos os cálculos de mecânica quân- 
Tabela 5.1: Cargas eletrônicas ( $q / e)$ e momento de dipolo ( $\mu / \mathrm{D})$ calculados (MP2/aug-cc-pVTZ) para uracila hidratada:

\begin{tabular}{crrrr}
\hline \hline Sítio & Gás & PCM $^{a}$ & ASEC-PCM $^{b}$ & ASEC-Iter $^{c}$ \\
\hline \hline $\mathbf{C}_{\mathbf{1}}$ & $-0,442653$ & $-0,514146$ & $-0,49560$ & $-0,520624$ \\
$\mathbf{C}_{\mathbf{2}}$ & 0,145221 & 0,195810 & 0,18354 & 0,242083 \\
$\mathbf{N}_{\mathbf{3}}$ & $-0,482560$ & $-0,530140$ & $-0,51721$ & $-0,579747$ \\
$\mathbf{C}_{\mathbf{4}}$ & 0,774494 & 0,862095 & 0,85267 & 0,947228 \\
$\mathbf{N}_{\mathbf{5}}$ & $-0,612821$ & $-0,662079$ & $-0,64031$ & $-0,719943$ \\
$\mathbf{C}_{\mathbf{6}}$ & 0,796301 & 0,873969 & 0,86072 & 0,947814 \\
$\mathbf{O}_{\mathbf{7}}$ & $-0,574893$ & $-0,684565$ & 0,69807 & $-0,743481$ \\
$\mathbf{O}_{\mathbf{8}}$ & $-0,566349$ & $-0,687029$ & $-0,70207$ & $-0,747134$ \\
$\mathbf{H}_{\mathbf{9}}$ & 0,167494 & 0,194621 & 0,19171 & 0,194351 \\
$\mathbf{H}_{\mathbf{1 0}}$ & 0,123306 & 0,157994 & 0,16131 & 0,141256 \\
$\mathbf{H}_{\mathbf{1 1}}$ & 0,328647 & 0,396739 & 0,40329 & 0,420206 \\
$\mathbf{H}_{\mathbf{1 2}}$ & 0,343813 & 0,396732 & 0,39999 & 0,417991 \\
$\langle\boldsymbol{\mu}\rangle$ & & & & \\
\hline \hline
\end{tabular}

(a) Resultado obtido para a geometria em vácuo. (b) Configuração média construída usando geometria e polarização PCM (veja o texto). (c) Resultado iterativo obtido para a geometria em vácuo, e polarização iterativa.

tica foram administrados dentro do formalismo GIAO (gauge independent atomic orbital) [84, 115, 116] como implementado no programa Gaussian 03 [118]. Todas as geometrias moleculares foram obtidas em nível de aproximação MP2/aug-cc-pVTZ. Finalmente, os resultados experimentais encontrados na literatura para a componente isotrópica do desvio químico $\left(\delta_{\text {iso }}\right)$ em oxigênio puderam ser diretamente comparados com a blindagem magnética nuclear $\left(\sigma_{i s o}\right)$ através de escala absoluta de conversão proposta por Wasylishen [119]:

$$
\sigma_{i s o}=287,5 \mathrm{ppm}-\delta_{i s o}
$$

\subsection{Resultados e discussões}

\section{Polarização do soluto}

Discutiremos inicialmente os efeitos do solvente sobre os momentos dipolares dos sistemas de interesse. Todos os cálculos (MP2/aug-cc-pVTZ) estão expostos nas tabelas 5.1 e 5.2 e mostram respectivamente os resultados obtidos para uracila e 5-fluorouracila.

O dipolo experimental para o gás de uracila é 3,87 D [117]. Os cálculos de Millefiore e 
Alparone reportam 4,29 e 4.33 D [120] para a molécula isolada. Já Ludwig e colaboradores [44] apontam um dipolo de 4,39 D. Nossos resultados estão em boa concordância com as predições anteriores, indicando um momento dipolar de 4,34 D. As estimativas teóricas discutidas indicam que pode haver algo de inconsistente com o experimento.

Modelos contínuos e discretos de solvente foram administrados a fim de estimar o momento de dipolo em solução aquosa. Considerando a geometria obtida em fase gasosa, o modelo contínuo polarizado (PCM) [25] prediz um dipolo de 6,03 D, implicando em um aumento de $39 \%$ relativo à molécula isolada.

Em um primeiro momento administramos o procedimento iterativo utilizando nas simulações MC geometria e cargas iniciais obtidas da molécula isolada. Essa predição é referenciada como ASEC-Iter e indica um dipolo de 6,45 D, o que representa um efeito de polarização de $51 \%$ relativo ao sistema isolado. A figura 5.2 mostra a evolução do momento de dipolo calculado como uma função do processo iterativo. Cada ponto em negrito é obtido com uma configuração média ASEC composta por 200 configurações MC sobrepostas. Por sua vez, cada estrutura MC é composta por um soluto explícito embebido em 300 moléculas de água representadas apenas pelas cargas pontuais inerentes a cada sítio atômico. Desse modo, uma configuração média ASEC é composta pela molécula de referência na presença de 180.000 cargas pontuais normalizadas pelo número de configurações. Esse modelo eletrostático permite uma redistribuição das cargas do soluto na presença do solvente, mas demanda um grande número de simulações MC.

Outra estratégia recentemente discutida $[45,121]$ consiste em obter geometria e cargas do soluto usando modelos contínuos de solvente e em seguida implementando esses parâmetros em uma simulação MC. Esse modelo tem sido usado com sucesso no estudo dos efeitos da polarização eletrônica do soluto sobre a blindagem magnética $\sigma\left({ }^{15} \mathrm{~N}\right)$ em pirimidina [121] e diazinas [45] solvatadas. Aqui esse modelo é referenciado como ASECPCM e indica um dipolo de $6,36 \mathrm{D}$, em boa concordância com a predição iterativa.

Para a 5-fluorouracila não há resultados experimentais para o gás. Nossos cálculos sobre o sistema isolado indicam um dipolo de 3,93 D. Em solução aquosa, o modelo contínuo prediz um dipolo de 5,60 D, o que implica em um efeito de polarização de $43 \%$ em relação ao gás. Já os modelos iterativos estão em boa concordância e indicam, respectivamente, dipolos de 5,92 e 5,91 D. 

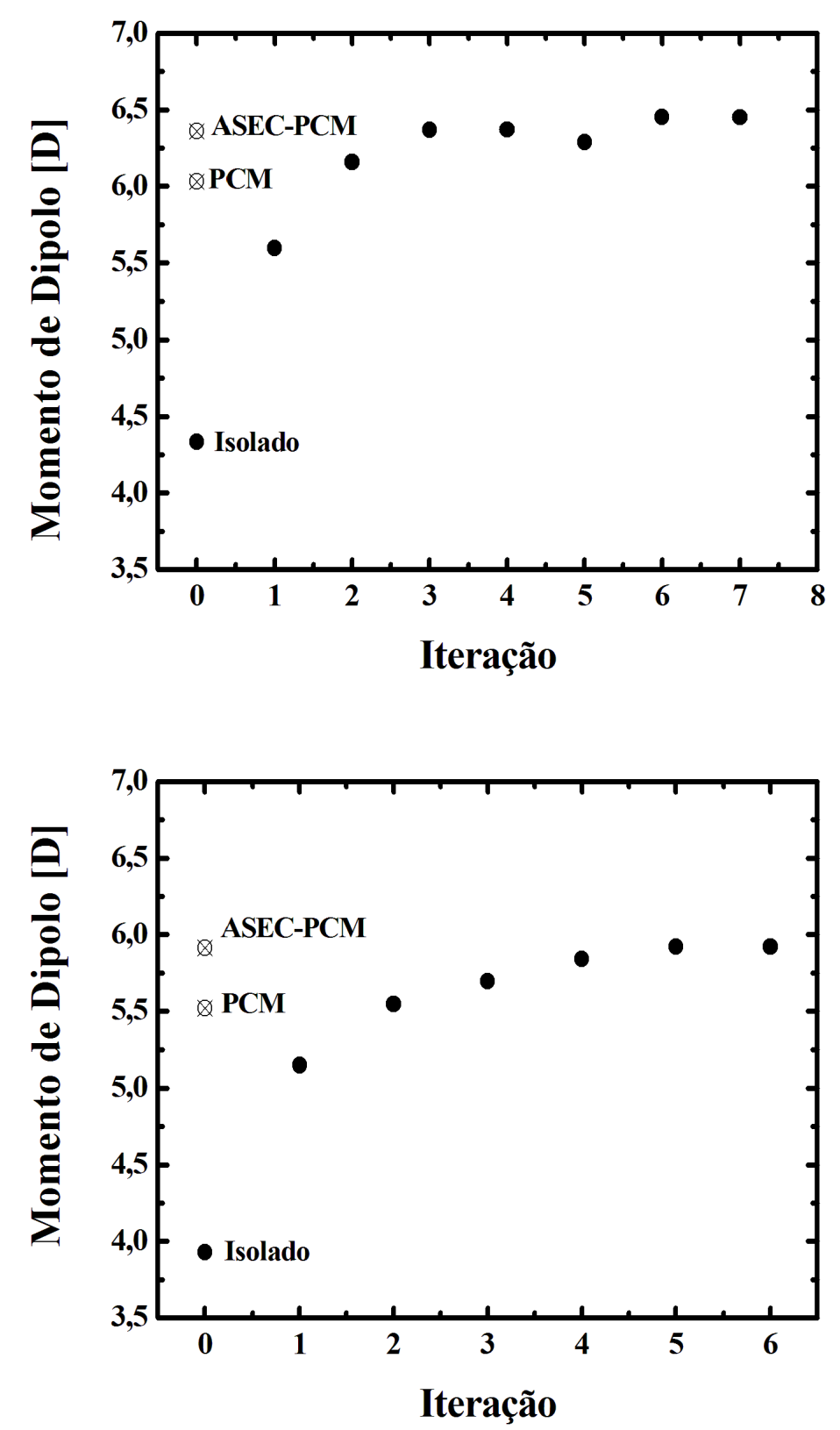

Figura 5.2: Convergência do momento de dipolo calculado (MP2/aug-cc-pVTZ) para uracila (topo) e 5-fluorouracila (abaixo) hidratadas versus o número de iterações. 
Tabela 5.2: Cargas eletrônicas ( $q / e)$ e momento de dipolo ( $\mu / \mathrm{D})$ calculados (MP2/aug-cc-pVTZ) para 5-fluorouracila hidratada:

\begin{tabular}{lrrrr}
\hline \hline Sítio & Gás & PCM $^{a}$ & ASEC-PCM $^{b}$ & ASEC-Iter $^{c}$ \\
\hline \hline $\mathbf{C}_{\mathbf{1}}$ & 0,05492 & 0,02517 & 0,03248 & $-0,00442$ \\
$\mathbf{C}_{\mathbf{2}}$ & 0,66287 & 0,73708 & 0,72814 & 0,79975 \\
$\mathbf{N}_{\mathbf{3}}$ & $-0,61033$ & $-0,65694$ & $-0,63843$ & $-0,71504$ \\
$\mathbf{C}_{\mathbf{4}}$ & 0,76053 & 0,84437 & 0,84068 & 0,92020 \\
$\mathbf{N}_{\mathbf{5}}$ & $-0,49195$ & $-0,53138$ & $-0,53087$ & $-0,57941$ \\
$\mathbf{C}_{\mathbf{6}}$ & $-0,01414$ & 0,02888 & 0,02895 & 0,08118 \\
$\mathbf{O}_{\mathbf{7}}$ & $-0,56663$ & $-0,67404$ & 0,68856 & $-0,72564$ \\
$\mathbf{O}_{\mathbf{8}}$ & 0,17559 & 0,21378 & 0,21478 & 0,19537 \\
$\mathbf{F}_{\mathbf{9}}$ & $-0,17099$ & $-0,19751$ & $-0,20304$ & $-0,18092$ \\
$\mathbf{H}_{\mathbf{1 0}}$ & $-0,50548$ & $-0,62209$ & $-0,63563$ & $-0,66999$ \\
$\mathbf{H}_{\mathbf{1 1}}$ & 0,35794 & 0,41671 & 0,41997 & 0,43945 \\
$\mathbf{H}_{\mathbf{1 2}}$ & 0,34766 & 0,41596 & 0,42162 & 0,43945 \\
$\langle\boldsymbol{\mu}\rangle$ & & & & 5,91 \\
\hline \hline
\end{tabular}

(a) Resultado obtido para a geometria em vácuo. (b) Configuração média construída usando geometria e polarização PCM (veja o texto). (c) Resultado iterativo obtido para a geometria em vácuo, e polarização iterativa.

\section{Blindagem magnética em uracila hidratada}

Discutiremos agora a blindagem magnética sobre o isótopo ${ }^{17} \mathrm{O}$ em uracila. A tabela 5.3 contém os principais resultados obtidos para a componente isotrópica $\sigma_{i s o}$ da blindagem magnética e para o desvio gás-líquido $\Delta \sigma_{i s o}$. Os resultados experimentais já se encontram convertidos para a escala absoluta da blindagem magnética segundo a eq. 5.1. Devido as performances similares dos funcionais utilizados (mPW1PW91 e B3LYP) na descrição, discutiremos principalmente os resultados obtidos em nível de aproximação mPW1PW91/aug-pcS-2. Utilizamos modelos contínuos e discretos de solvente para descrever os efeitos do meio sobre as propriedades de interesse.

A uracila possui dois oxigênios não simétricos experimentando diferentes ambientes químicos (veja figura 5.1). Essa característica origina dois picos distintos no espectro RMN desse composto. Não há na literatura informações experimentais sobre a blindagem magnética para o gás, mas nossos cálculos estimam blindagens de $-7,8$ e $-113,5$ ppm sobre $\mathrm{O}_{7}$ e $\mathrm{O}_{8}$, respectivamente.

Resultados experimentais para a molécula hidratada à $95^{\circ} \mathrm{C}$, nesta ordem, indicam blindagens de 55, 5 e -13, 5 ppm sobre $\mathrm{O}_{7}$ e $\mathrm{O}_{8}$ [98]. O modelo contínuo polarizado prediz blindagens de 35,5 e -41,1 ppm. Note que essa aproximação subestima em excesso a 


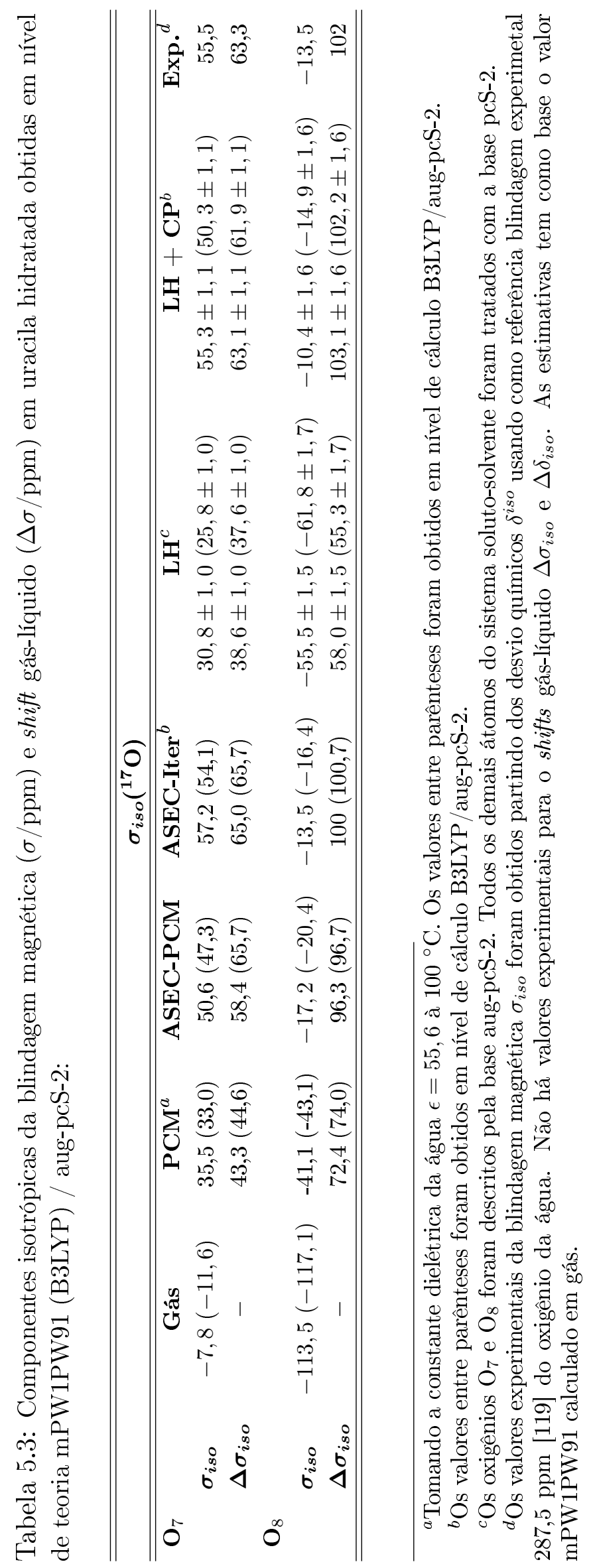


propriedade de interesse, divergindo do experimento por $\sim 20 \mathrm{ppm}$.

Utilizamos o procedimento iterativo para estudar a blindagem magnética. Os resultados obtidos com esse modelo estão referenciados como ASEC-Iter e predizem por sua vez, blindagens de 57,2 e -13,5 ppm sobre $\mathrm{O}_{7}$ e $\mathrm{O}_{8}$. A predição iterativa está em excelente concordância com o experimento. A figura 5.3 mostra a evolução da blindagem magnética sobre $\mathrm{O}_{8}$ como função do processo de polarização do soluto. Como no momento de dipolo, uma convergência suave é obtida quando as cargas eletrônicas entram em equilíbrio com o solvente.

Em termos da migração das cargas eletrônicas do soluto devido à polaridade do solvente, Witanowiski e colaboradores têm observado e discutido esse efeito em vários sistemas nitrogenados $[9,10,11,12,13,14]$. Segundo Witanowski, a presença do solvente força a redistribuição das cargas do soluto e o acúmulo de cargas sobre determinados sítios favorece um shift positivo $\left(\Delta \sigma_{i s o}>0\right)$ na blindagem magnética em relação à molécula isolada. Nossos resultados corroboram a proposta de Witanowski. Comparando as cargas eletrônicas obtidas para os oxigênios da uracila isolada e hidratada (veja a tabela 5.3) observa-se que a polarização do soluto aumenta a população eletrônica sobre esses sítios. Esse aumento de cargas supostamente contribui para o aumento da blindagem magnética nuclear $\sigma\left({ }^{17} \mathrm{O}\right)$, causando o efeito downfield. A figura 5.4 mostra a relação linear entre a blindagem magnética e as cargas eletrônicas acumuladas no núcleo de interesse.

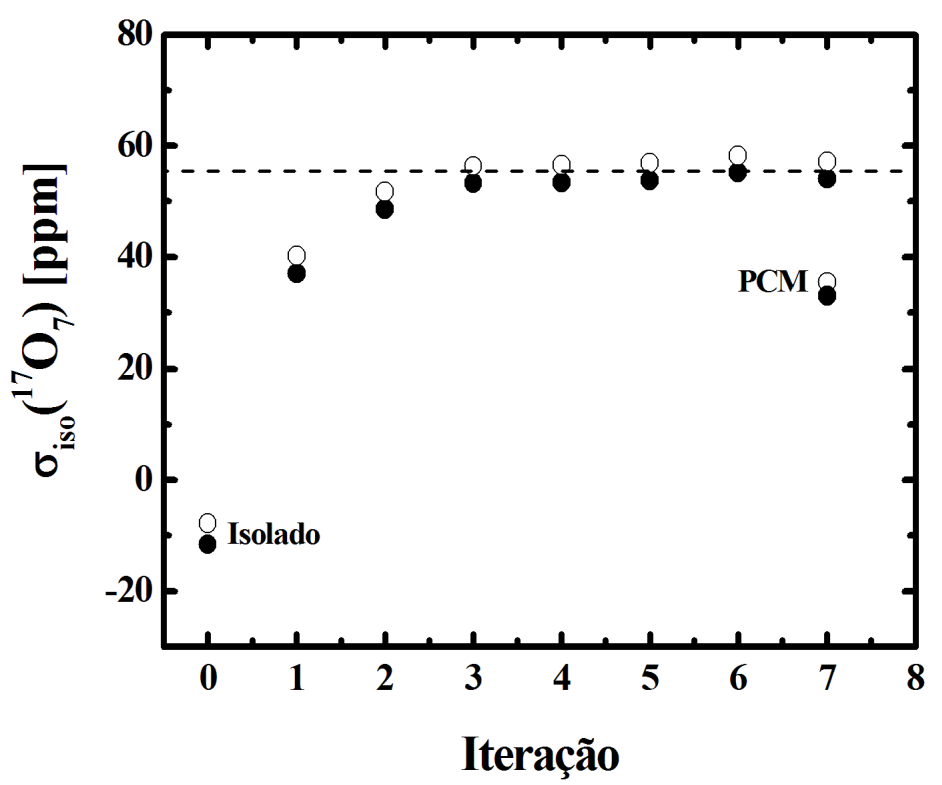

Figura 5.3: Componente isotrópica da blindagem magnética $\sigma\left({ }^{17} \mathrm{O}_{7}\right)$ em uracila hidratada. O círculos em negrito e vazados foram obtidos com os funcionais B3LYP e mPW1PW91, respectivamente. A linha tracejada corresponde ao resultado experimental. 


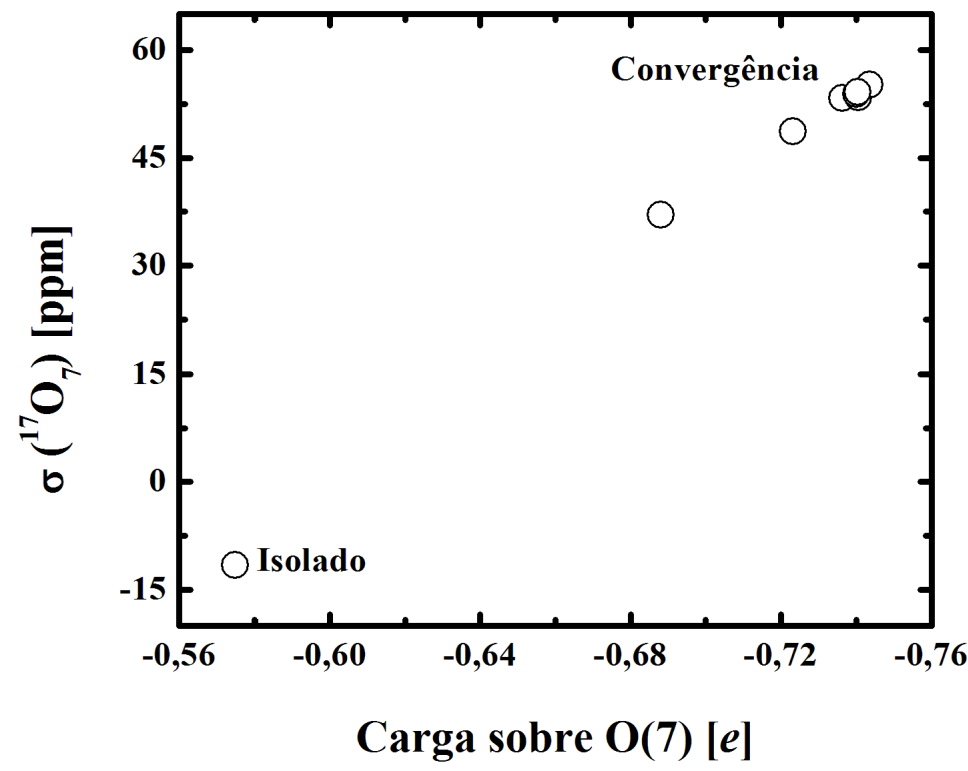

Figura 5.4: Blindagem magnética nuclear sobre o sítio $\mathrm{O}_{7}$ em função da população eletrônica. Blindagem magnética e e cargas são simultaneamente calculados em cada passo iterativo.

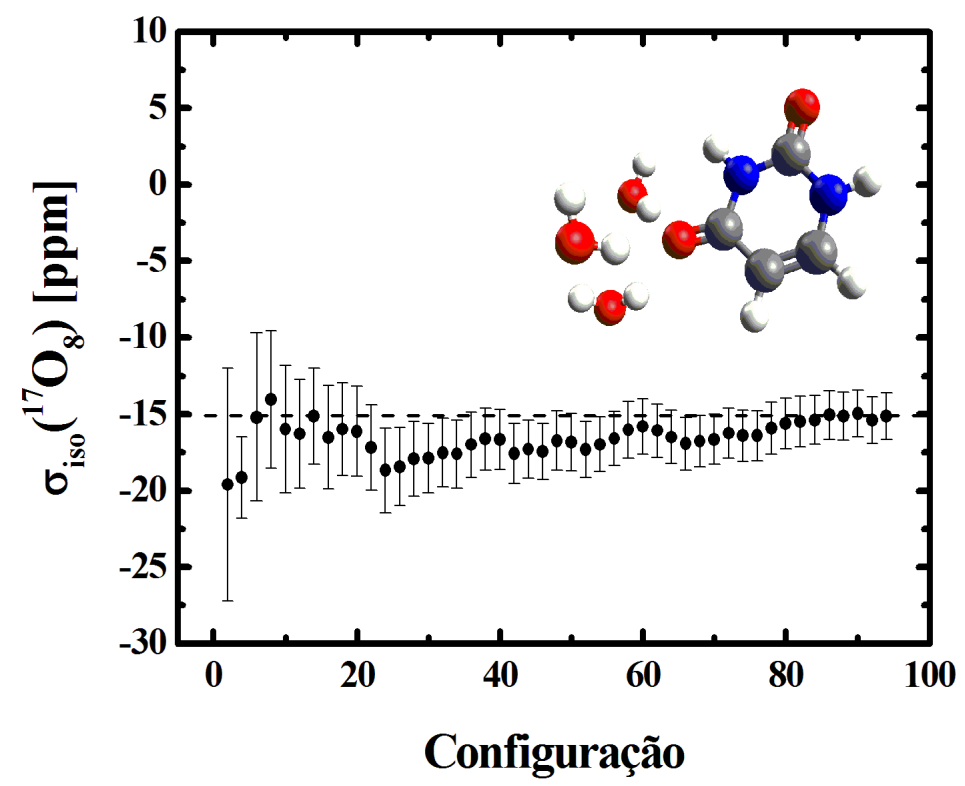

Figura 5.5: Convergência para o blindagem magnética nuclear $\sigma_{i s o}\left({ }^{17} \mathrm{O}_{8}\right)$ em uracila hidratada. A linha tracejada representa o valor médio $14,9,4 \pm 1,6 \mathrm{ppm}$ obtido.

O método iterativo, como aplicado acima, implica em um número razoável de simulações MC. Recentemente propusemos uma alternativa que representa uma economia na quantidade de simulações [45, 121]. Ao invés de usar geometria e cargas obtidas em vácuo, 
usam-se modelos contínuos para obter a geometria e cargas do soluto. Em seguida, esses parâmetros são implementados em uma única simulação $\mathrm{MC}$, na qual uma nova configuração média ASEC é gerada e usada para calcular as propriedades de interesse. Não há necessidade de uma nova polarização iterativa, uma vez que o sistema já foi polarizado usando o modelo contínuo. Temos mostrado que os resultados obtidos com esse modelo reproduzem muito bem o experimento [45, 121]. Referenciamos esse modelo como ASEC-PCM, predizendo blindagens de 50,6 e -17,2 ppm sobre $\mathrm{O}_{7}$ e $\mathrm{O}_{8}$, respectivamente.

Os modelos usados até o momento tiram vantagem exclusivamente de interações eletrostáticas. Avaliamos os efeitos do solvente explícito considerando a formação de ligações de hidrogênio $\mathrm{O} \cdots \mathrm{HOH}$ entre uracila e água. Esse modelo é descrito no texto simplesmente como LH. Com essa aproximação obtivemos blindagens de 30,8 ppm sobre $\mathrm{O}_{7}$ e $-55,5 \mathrm{ppm}$ para $\mathrm{O}_{8}$. Note que esses resultados representam uma descrição muito pobre do experimento. Outra alternativa, computacionalmente mais cara, consiste em combinar interações específicas soluto-solvente com interações eletrostáticas provenientes do bulk. Esse modelo é descrito aqui como $\mathrm{LH}+\mathrm{CP}$ e prediz $55,3 \pm 1,1 \mathrm{ppm}$ sobre $\mathrm{O}_{7} \mathrm{e}-10,4 \pm 1,6$ ppm sobre $\mathrm{O}_{8}$, em boa concordância com o experimento. A figura 5.5 mostra a quantidade de cálculos de mecânica quântica necessários para obter a convergência da blindagem $\sigma\left({ }^{17} \mathrm{O}_{8}\right)$ usando o modelo LH + CP. Finalmente, é interessante observar a sensibilidade da blindagem $\sigma\left({ }^{17} \mathrm{O}\right)$ com relação ao meio solvente. Tomando o modelo $\mathrm{LH}+\mathrm{CP}$, por exemplo, vemos que shift $\Delta \sigma_{i s o}$ causado pela presença do solvente é da ordem de $\sim 63 \mathrm{ppm}$ em $\mathrm{O}_{7}$ e $\sim 103 \mathrm{ppm}$ em $\mathrm{O}_{8}$. A figura 5.6 ilustra brevemente os modelos discretos e explícitos de solvente utilizados. 


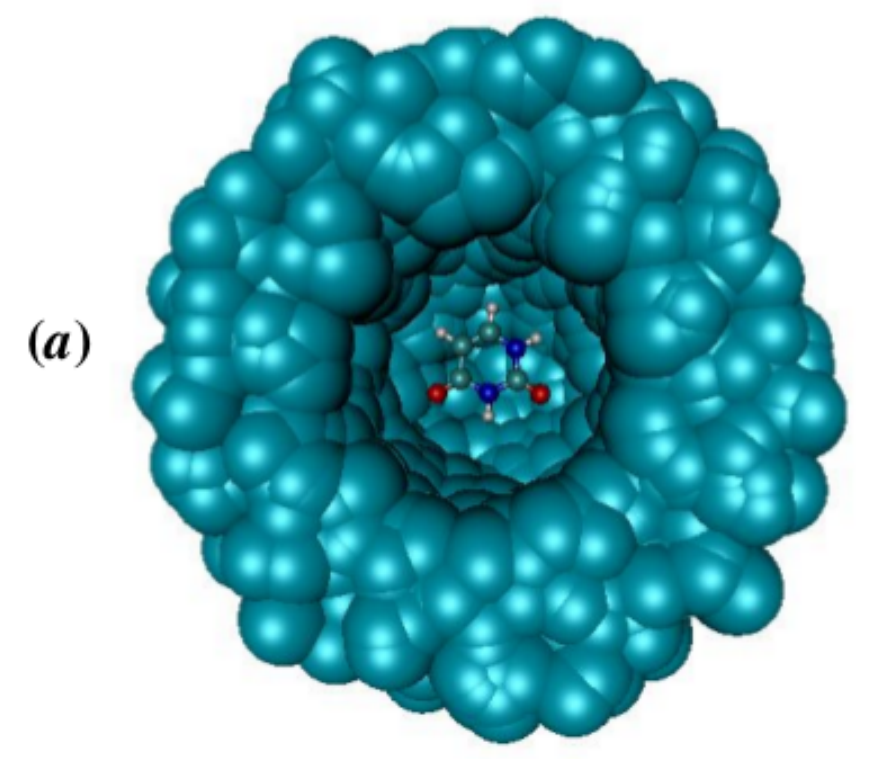

(b)

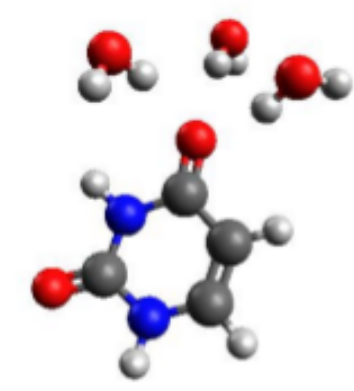

(c)

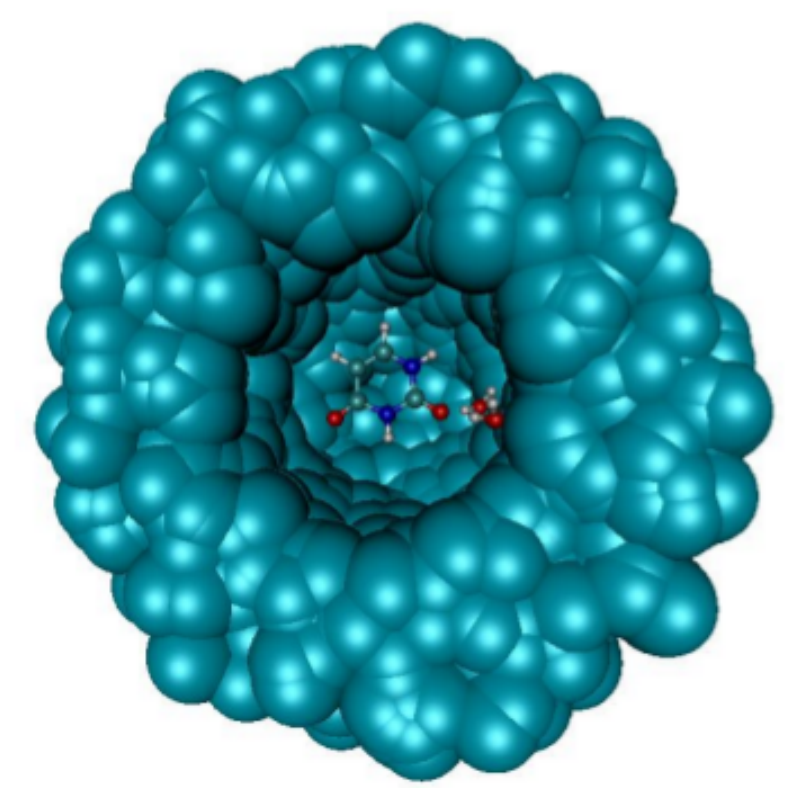

Figure 5.6: (a) Configuração média ASEC obtida a partir das simulações clássicas MC. (b) Típica formação de ligações de hidrogênio em uracila-água. (c) Estrutura soluto-solvente formando ligações de hidrogênio em água embebida no campo eletrostático das águas remanescentes. 
Tabela 5.4: Componentes isotrópicas da blindagem magnética $(\sigma / \mathrm{ppm})$ e shift gás-líquido $(\Delta \sigma / \mathrm{ppm})$ em 5-fluorouracila hidratada. Os valores teóricos foram obtidos em nível de aproximação mPW1PW91(B3LYP)/aug-pcS-2:

\begin{tabular}{lcccccr}
\hline \hline \multicolumn{6}{c}{$\boldsymbol{\sigma}_{\text {iso }}\left({ }^{\mathbf{1 7}} \mathbf{O}\right)$} \\
\hline \hline $\mathbf{O}_{7}$ & & Gás & PCM $^{a}$ & ASEC-PCM & ASEC-Iter $^{b}$ & Exp. $^{c}$ \\
& $\boldsymbol{\sigma}_{\text {iso }}$ & $3,6(-0,2)$ & $43,7(41,0)$ & $49,4(45,9)$ & $56,5(53,3)$ & 57,5 \\
& $\boldsymbol{\Delta} \boldsymbol{\sigma}_{\text {iso }}$ & - & $40,1(41,3)$ & $45,8(46,2)$ & $52,9(53,5)$ & \\
$\mathbf{O}_{8}$ & & & & & & \\
& $\boldsymbol{\sigma}_{\text {iso }}$ & $-93,5(-96,4)$ & $-25,5(-27,0)$ & $-13,6(-16,4)$ & $-12,6(-15,1)$ & $-6,5$ \\
& $\boldsymbol{\Delta} \boldsymbol{\sigma}_{\text {iso }}$ & - & $68,0(69,4)$ & $79,9(80,0)$ & $81,0(81,3)$ & \\
\hline \hline
\end{tabular}

(a) Tomando a constante dielétrica da água $\epsilon=55,6$ à $100{ }^{\circ} \mathrm{C}$. Os valores entre parênteses foram obtidos em nível de cálculo B3LYP/aug-pcS-2. (b) Os valores entre parênteses foram obtidos em nível de cálculo B3LYP/aug-pcS-2. (c) Os valores experimentais da blindagem magnética $\sigma_{i s o}$ foram obtidos partindo dos desvio químicos $\delta^{i s o}$ usando como referência blindagem experimetal 287,5 ppm [119] do oxigênio da água. Não há valores experimentais para o shifts gás-líquido $\Delta \sigma_{i s o}$ e $\Delta \delta_{i s o}$. As estimativas tem como base o valor mPW1PW91 calculado em gás.

\section{Blindagem magnética em 5-fluorouracila hidratada}

Assim como em uracila, obtivemos resultados satisfatórios somente considerando os efeitos da polarização induzida pelo solvente e contribuições eletrostáticas provenientes do bulk, por isso não executamos cálculos com solvente explícito. Discutimos neste momento a blindagem magnética em 5-fluorouracila sob a ótica dos modelos eletrostáticos contínuos e discretos de solvente. Os resultados obtidos estão dispostos na tabela 5.4.

Assim como em uracila, não há resultados experimentais para o gás. Contudo, nossos cálculos estimam blindagens de 3,6 ppm sobre $\mathrm{O}_{7}$ e $-93,5$ ppm sobre $\mathrm{O}_{8}$ para o sistema isolado. Experimentos realizados em água reportam blindagens de 57,5 e -6,5 ppm sobre $\mathrm{O}_{7}$ e $\mathrm{O}_{8}$, respectivamente [98]. Novamente o modelo contínuo PCM não descreve bem as constantes magnéticas de interesse. Sobre $\mathrm{O}_{7}$ obtivemos uma blindagem de 43,7 ppm, e $-25,5 \mathrm{ppm}$ sobre $\mathrm{O}_{8}$. O modelo ASEC-PCM descrito anteriormente se comporta melhor e prediz constantes magnéticas de 49,4 e -13,6 ppm sobre $\mathrm{O}_{7}$ e $\mathrm{O}_{8}$, respectivamente. A melhor descrição é obtida com o modelo iterativo ASEC-Iter. Sobre $\mathrm{O}_{7}$ obtivemos uma blindagem de 56,5 ppm, já sobre $\mathrm{O}_{8}$ nosso modelo prediz uma constante magnética de $-6,5 \mathrm{ppm}$. 


\subsection{Conclusões}

Analisamos a influência da polarização eletrônica induzida pelo solvente e seus efeitos sobre a blindagem magnética nuclear $\sigma\left({ }^{17} \mathrm{O}\right)$ em uracila e 5-fluorouracila hidratadas. Considerando os sistemas isolados, obtivemos dipolos de 4,34 D para a uracila e 3,93 D para a 5-fluouracila. Em água esses valores variam de 6,03 até $6,45 \mathrm{D}$ para a uracila e de 5,60 até 5,92 D para a 5-fluorouracila. Esses resultados mostram uma polarização considerável.

Constantes magnéticas nucleares são propriedades locais. Isso significa que é suficiente descrever rigorosamente apenas a região de interesse. Como os sítios de interesse são aceitadores em ligações de hidrogênio, aferimos o papel da formação dessas ligações sobre $\sigma\left({ }^{17} \mathrm{O}\right)$. Observamos que apenas a inclusão explícita do solvente não é suficiente para entender o espectro RMN. Com essa aproximação obtivemos desvios de até $\sim 40$ ppm em relação ao experimento. Melhores resultados foram obtidos incluindo interações eletrostáticas. Neste modelo, o soluto eletronicamente polarizado ainda interage com o solvente através de ligações de hidrogênio, mas agora a supermolécula (soluto-solvente) é embebida no banho eletrostático das moléculas remanescentes consideradas agora como cargas pontuais. Esse modelo proporciona uma descrição mais fiel do experimento, apresentando desvios de apenas $\sim 2$ ppm. Esses aspectos indicam que interações eletrostáticas provenientes do bulk têm papel fundamental na descrição de propriedades magnéticas nucleares.

A discussão acima indica que a polarização induzida pelo meio é crucial no trato de propriedades magnéticas nucleares. Utilizamos modelos contínuos, bem como um procedimento iterativo para aferir esses efeitos. De modo geral, observamos que modelos contínuos apresentam uma descrição pobre das constantes RMN. O modelo iterativo utilizado é também um método sofisticado que permite equilibrar as cargas do soluto na presença do solvente. As predições feitas com esse procedimento concordam muito bem com o experimento, apresentando desvios de apenas $\sim 3 \mathrm{ppm}$.

O método iterativo também permite analisar a convergência da blindagem magnética em função da redistribuição de cargas eletrônicas no sítio de interesse. Para ambos os oxigênios em uracila e 5-fluorouracila, observamos que a polarização eletrônica induzida pelo solvente torna o sistema mais blindado. Constatamos também que o aumento na blindagem magnética está linearmente relacionado com a migração de cargas eletrônicas em direção aos sítios $\mathrm{O}_{7}$ e $\mathrm{O}_{8}$. Isso significa que a blindagem magnética nuclear é um ótimo sensor para aferir a redistribuição das cargas eletrônicas do soluto, já que essa última grandeza é uma consequência direta da polarização devido ao meio e não pode ser medida experimentalmente. Observamos que $\sigma\left({ }^{17} \mathrm{O}\right)$ é uma propriedade molecular extremamente sensível ao meio, na qual shifts $\Delta \sigma\left({ }^{17} \mathrm{O}\right)$ de até 100 ppm são reportados. 


\section{Capítulo 6}

\section{Piridina hidratada}

\subsection{Introdução}

Constantes magnéticas nucleares em nitrogênio têm despertado cada vez mais interesse. Piridina, por exemplo, tem sido foco de vários estudos teóricos [122, 123, 124, 125, 126] e experimentais $[9,15,127,128,129]$. Constantes RMN são extremamente sensíveis à mudanças estruturais e ao ambiente químico, assim esses sistemas são excelentes laboratórios para testar e desenvolver novas metodologias. Para o estudo de propriedades magnéticas nucleares é importante entender o papel desenvolvido pelo solvente. Em água, por exemplo, piridina forma ligações de hidrogênio, as quais reconhecidamente têm grande influência sobre a blindagem magnética $\sigma\left({ }^{15} \mathrm{~N}\right)$. Os efeitos da polarização eletrônica sobre o soluto têm sido frequentemente referenciados por Witanowski [9, 10, 11, 12, 13, 14], mas pouco se sabe sobre a influência dos efeitos da relaxação geométrica induzidos pelo solvente. Variações na conformação molecular têm relevância em RMN, uma vez que importantes propriedades moleculares como o acoplamento indireto entre spins nucleares, mostram extrema sensibilidade a rearranjos intramoleculares.

Há na literatura inúmeros trabalhos versando sobre piridinas, mas aqui, discutiremos principalmente os efeitos estruturais induzidos pelo meio e veremos que suas contribuições para a blindagem magnética nuclear $\sigma\left({ }^{15} \mathrm{~N}\right)$ são pequenas. Desse modo, ao longo do texto, manteremos o foco na polarização eletrônica do soluto devido ao solvente. Como primeira aproximação, assumiremos que a polarização recíproca do solvente, devido ao soluto, é pequena e não será considerada. A polarização eletrônica do soluto pode ser incluída usando um método iterativo [47, 48]. Discutiremos dois processos iterativos distintos, um relaxando somente a distribuição de cargas do soluto e, outro, relaxando também sua geometria. Assim, veremos que é possível comparar os resultados envolvendo as geometrias rígidas e relaxadas do soluto e quantificar suas contribuições. 


\subsection{Detalhes computacionais}

Um procedimento sequencial QM/MM foi utilizado para estudar as blindagens magnéticas em piridina hidratada. Nessa aproximação, primeiro as configurações do líquido são geradas usando simulações clássicas Monte Carlo. Posteriormente, algumas dessas configurações, estatisticamente descorrelacionadas, são selecionadas e submetidas a cálculos de mecânica quântica. Todas as simulações MC foram executadas utilizando o programa DICE [101] e executadas no ensemble $N P T$ com, $T=25^{\circ}$ e $P=1 \mathrm{~atm}$ e com uma molécula do soluto diluída em 900 águas. As interações intermoleculares foram modeladas pelos potenciais de Lennard-Jones (LJ) e Coulomb. Para as moléculas de água usamos os parâmetros TIP3P [102]. Para o soluto, os parâmetros LJ foram extraídos do campo de forças OPLS [79], mas as cargas atômicas foram obtidas em ambiente líquido a fim de considerar os efeitos da polarização do soluto. Esses efeitos são incluídos através de um procedimento iterativo [47, 48], no qual é permitido ao soluto relaxar ambos, geometria e cargas, na presença do solvente. As cargas atômicas são obtidas em nível de aproximação MP2/aug-cc-pVTZ utilizando procedimento CHELPG [103], o qual ajusta o potencial clássico ao quântico. As novas cargas posicionadas nos sítios atômicos do soluto são então implementadas em uma nova simulação MC. Note que o procedimento permite um rearranjo do solvente devido à nova distribuição de cargas do soluto. Ao longo do processo iterativo, os cálculos de mecânica quântica são executados usando uma configuração eletrostática média do solvente (ASEC - average solvent electrostatic configuration) [48]. Para construir uma configuração ASEC, sobrepusemos 250 configurações MC estatisticamente descorrelacionadas, de modo que cada configuração é composta por uma molécula do soluto embebida no campo eletrostático de 300 moléculas de água representadas apenas como cargas pontuais. Isso significa que foram incluídas todas as interações eletrostáticas dentro de $11 \AA$.

Os efeitos de relaxação de geometria foram inferidos através do método do gradiente de energia livre (FEG - free energy gradiente) [130, 131, 132] em conjunto ao procedimento QM/MM. Na prática, após cada passo do processo iterativo, temos uma nova função de onda para o soluto, incluindo interações eletrostáticas com o solvente. Então calculamos as derivadas primeira e segunda da energia com respeito às posições nucleares do soluto, na presença do solvente eletrostaticamente considerado. Essas energias são então implementadas no esquema Quasi-Newton (aqui usamos o algorítimo Broyden-FletcherGoldfarb-Shanno [133, 134, 135, 136, 137] implementado no programa GAUSSIAN 03 [118]) para obter a nova conformação molecular do soluto. A nova conformação do soluto é usada para recalcular as cargas atômicas, por meio da configuração ASEC e ambas, cargas e geometria, são reimplementados em uma nova simulação MC. O processo é repetido até a convergência do momento de dipolo e dos parâmetros geométricos. 
Detalhes sobre a aproximação FEG são bem descritos por Nagaoka e colaboradores [130, 131, 132] e foram recentemente implementados em um programa chamado Diceplayer [138], o qual faz uma interface entre o programa de simulações MC Dice [101] e programas de mecânica quântica. Com o uso da aproximação FEG, resultados para o acoplamento indireto entre spins nucleares em amônia líquida têm sido obtidos em boa concordância com o experimento [139]. Essa aproximação tem sido usada também por Aguilar e colaboradores [140, 141] para inferir o efeito do solvente sobre a estrutura do soluto.

Os desvios químicos experimentais $\delta\left({ }^{15} \mathrm{~N}\right)$ foram convertidos para escala da blindagem magnética $\sigma\left({ }^{15} \mathrm{~N}\right)$, usando a blindagem magnética do nitrogênio em nitrometano $(-135,8$ ppm) como referência [126]. Considerando correções para a susceptibilidade magnética do bulk, Duthaler e Roberts [66] reportaram -84, 4 ppm como a blindagem magnética para o nitrogênio da piridina gasosa. Como experimentos de ressonância magnética nuclear são complicados de serem executados em moléculas isoladas, é comum o uso de ciclohexano como solvente para aproximar as condições de vácuo. Em ciclohexano, Duthaler e Roberts também reportaram-82,9 ppm, depois de considerar efeitos de bulk e extrapolação com relaxão à diluição infinita. Contudo, comparações com resultados teóricos para piridina isolada mostram discrepâncias. Nosso resultado atual, usando o modelo B3LYP e as bases aug-pcS- $n(n=1,2,3)$ [114], construídas para o estudo the propriedades magnéticas, mostram resultados para $\sigma\left({ }^{15} \mathrm{~N}\right)$, variando entre $-110,2$ e $-117,2 \mathrm{ppm}$. Estes resultados estão muito afastados do experimento realizado para o gás [66], com diferenças variando entre 25,8 e 32,8 ppm. Esse desacordo sugere uma comparação com outros métodos de química quântica. Portanto, também estudamos o sistema isolado usando as aproximações RPA (random phase approximation) [142] e SOPPA (second-order polarization propagator aproximation) [143] como implementado no programa DALTON [144]. Por exemplo, usando as bases aug-pcS-2 [114] e aug-cc-pVTZ-J [67, 68], nosso resultado RPA prediz constantes de blindagem de $-115,8$ e $-104,7$ ppm, respectivamente e usando a aproximação SOPPA/aug-cc-pVTZ-J, obtivemos -103, 6 ppm. Esses resultados diferem apreciavelmente do experimento. Mennucci e colaboradores [37] recentemente obtiveram em nível de cálculo B3LYP $/ 6-311+\mathrm{G}(d, p)$ a blindagem $\sigma\left({ }^{15} \mathrm{~N}\right)$ de $-102,8$ ppm em piridina isolada. Obtivemos $-103,5 \mathrm{ppm}$, usando o mesmo nível de teoria. Os resultados discutidos acima indicam inconsistências entre teoria e os experimentos realizados sobre o sistema isolado. Portanto, neste estudo reportaremos apenas os resultados calculados em ambiente aquoso.

DFT e funções base apropriadas têm sido exaustivamente explorados no cálculo de blindagens magnéticas e acoplamentos indiretos entre spins nucleares [121, 108, 105, 145, 146]. Aqui empregamos o mesmo modelo B3LYP $/ 6-311+\mathrm{G}(d, p)$ utilizado com sucesso 
por Mennucci et al [37], usando a aproximação GIAO (gauge independent atomic orbital) [84, 115, 116] para calcular as blindagens magnéticas nucleares em piridina hidratada, como implementado no programa GAUSSIAN 03 [118].

\subsection{Resultados e discussões}

\section{Polarização do soluto}

A tabela 6.2 mostra os principais resultados obtidos para o momento de dipolo da piridina isolada e hidratada. O valor 2,33 D calculado em nível de aproximação MP2/aug-ccpVTZ está em boa concordância com o resultado experimental de 2,15 \pm 0,05 D [147]. A geometria obtida em mesmo nível de teoria para o sistema isolado, também mostra boa concordância com o experimento. A ligação $\mathrm{N}_{1}-\mathrm{C}_{2}$ calculada é $1,341 \AA$, enquanto os comprimentos $\mathrm{C}_{2}-\mathrm{C}_{3}$ e $\mathrm{C}_{3}-\mathrm{C}_{4}$ são 1,393 e 1,391 $\AA$, respectivamente. Os valores

experimentais reportados para essas ligações são $1,340 \AA, 1,395 \AA$ e $1,394 \AA$ [148] (os índices atômicos são mostrados na figura 6.1).

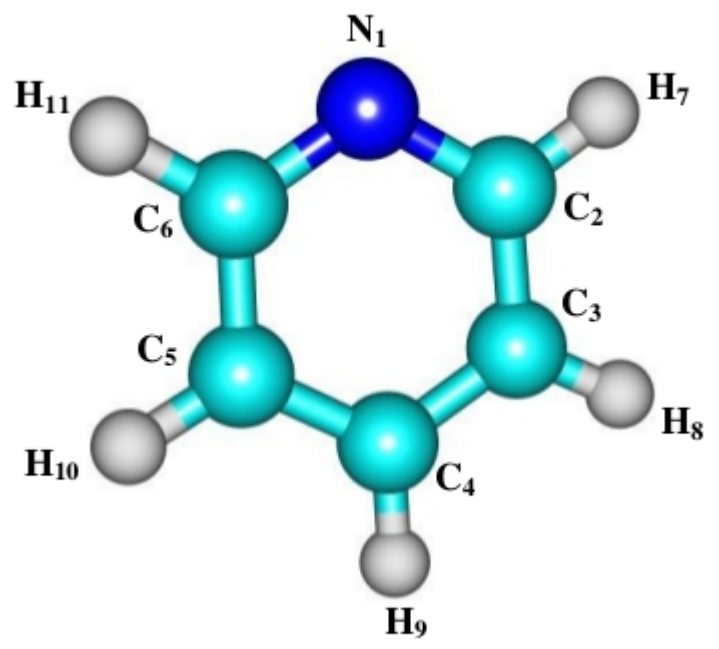

Figura 6.1: Piridina.

Dados experimentais sobre o momento de dipolo molecular em fase líquida são escassos devido a dificuldade natural de uma medida direta. Resultados teóricos podem ser encontrados somente para aglomerados [149, 150]. Aqui investigamos a polarização do soluto devida ao solvente, o que implica no aumento do momento de dipolo molecular. Os efeitos da polarização foram considerados usando modelos contínuos e discretos de solvente. A aproximação contínua é representada em termos do modelo contínuo polarizado 
[25] (PCM), enquanto a aproximação discreta descreve o solvente somente como cargas pontuais. A polarização iterativa é usada aqui com e sem relaxação de geometria, como descrito na seção anterior. A convergência calculada para o caso rígido é mostrada na figura 6.2. O modelo PCM obtém um momento de dipolo de 3,94 D, o que representa um aumento de $46 \%$ comparado com resultado reportado para o gás. Com o esquema iterativo representando o solvente como cargas pontuais, obtivemos momentos de dipolo de 3,94 (aumento de 69\%) e 4,38 D (aumento de 88\%) para as geometrias rígida e relaxada, respectivamente. As mudanças geométricas são suaves, como discutido anteriormente em moléculas similares contendo nitrogênio [151], mas mesmo assim afetam consideravelmente o momento de dipolo calculado. Analisaremos a seguir a influência dessa polarização sobre as ligações de hidrogênio soluto-solvente, bem como sobre a redistribuição de cargas sobre o nitrogênio da piridina e como esses fatores afetam $\sigma\left({ }^{15} \mathrm{~N}\right)$ em água.

Tabela 6.1: Momento de dipolo $(\mu / \mathrm{D})$ da piridina calculado em nível de teoria MP2/aug-ccpVTZ:

\begin{tabular}{|c|c|c|c|c|}
\hline \multicolumn{2}{|r|}{ Isolada } & \multicolumn{3}{|c|}{ Hidratada } \\
\hline & Exp.[147] & PCM & Discreto (Rígido) & Discreto (Relaxado) \\
\hline$\mu$ & $2,15 \pm 0,05$ & 3,41 & 3,94 & 4,38 \\
\hline
\end{tabular}

\section{Ligações de hidrogênio}

A partir do modelo rígido da piridina, discutiremos brevemente os efeitos da polarização sobre a coordenação do solvente ao redor do soluto. Na situação não polarizada, obtivemos uma coordenação de 1,62 moléculas de água. No caso polarizado, o número de coordenação muda para 2,02. Usando somente critérios geométricos, não se pode garantir que todas as moléculas do solvente formam ligações de hidrogênio com o soluto. Assim, usamos também critérios energéticos derivados da energia de interação entre pares (veja figura 6.3) $[149,152,153]$. Assim, para o caso polarizado, consideramos uma formação de pontes de hidrogênio quando $R_{\mathrm{N}-\mathrm{O}} \leq 3,5 \AA$, o ângulo $\alpha(\mathrm{N}-\mathrm{OH}) \leq 35^{\circ}$ e energias de interação menores que $-4,0 \mathrm{kcal} / \mathrm{mol}$ são obtidos. Os critérios geométricos são extraídos das funções de distribuição radial e angular, já o critério de energia, é obtido diretamente da distribuição de energias de interação entre pares mostrada na figura 6.3. 


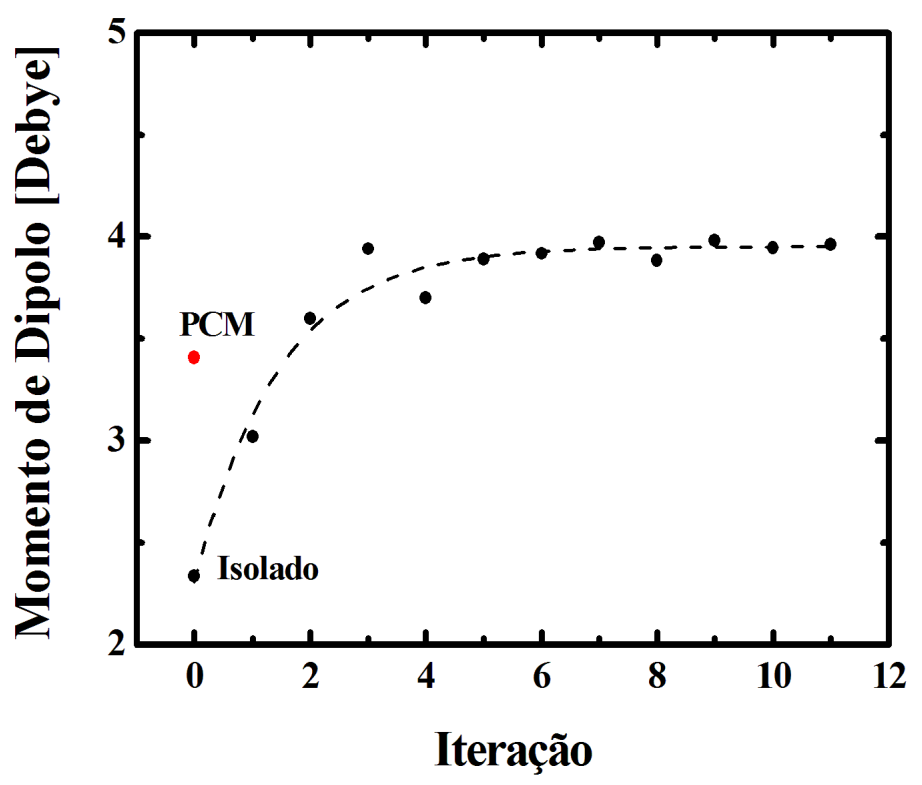

Figura 6.2: Momento de dipolo calculado para piridina isolada e hidratada. O círculo cheio em vermelho mostra a predição do modelo contínuo polarizado (PCM). Os pontos escuros mostram a evolução do momento de dipolo, partindo de seu valor para o sistema isolado até a convergência dessa propriedade como uma função do procedimento iterativo.

Tabela 6.2: Estatística da formação de ligações de hidrogênio entre piridina e água:

\begin{tabular}{|c|c|c|c|}
\hline \multirow[b]{2}{*}{ Ligações } & \multicolumn{3}{|c|}{ Ocorrência (\%) } \\
\hline & Ref. [150] & Não Polarizado $^{a}$ & Polarizado $^{a}$ \\
\hline 0 & 17 & 36 & 2 \\
\hline 1 & 62 & 56,8 & 36,4 \\
\hline 2 & 20 & 7,2 & 60,4 \\
\hline 3 & 1 & 0 & 1,2 \\
\hline Média & 1.1 & 0,71 & 1,61 \\
\hline
\end{tabular}

( a) Ambos os modelos polarizado e não-polarizado usam a geometria rígida de piridina. 250 configurações estatisticamente descorrelacionadas foram analisadas.

Uma análise similar é feita para o caso não polarizado, no qual $R_{\mathrm{N}-\mathrm{O}} \leq 3,2 \AA, \alpha(\mathrm{N}-\mathrm{OH}) \leq$ $35^{\circ}$ e energias de interação menores que $-3,0 \mathrm{kcal} / \mathrm{mol}$ são obtidos. No caso polarizado, observamos que em $2 \%$ das configurações não há formação de ligações de hidrogênio, enquanto que $36 \%$ fazem apenas uma ligação. A situação mais provável corresponde ao caso em que 60\% das configuraçõe fazem duas ligações de hidrogênio. Na média, como pode ser visto na tabela 6.2, obtivemos 1,6 ligações de hidrogênio envolvendo o nitrogênio da piridina e os átomos de hidrogênio da água. Esses valores são consideravelmente maiores 
que os obtidos para o caso não polarizado (0,71 ligações de hidrogênio) e também ligeiramente maiores que os valores obtidos ao se considerar as cargas do modelo OPLS $(1,1$ ligações de hidrogênio).

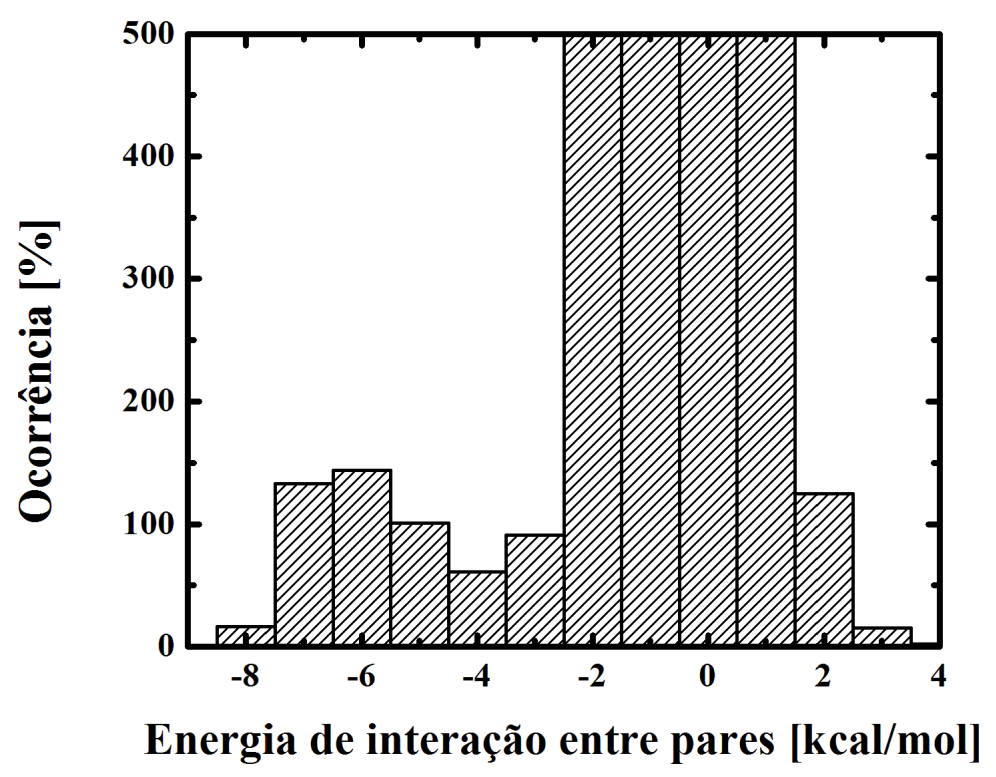

Figura 6.3: Histograma da energia de interação entre pares piridina-água, obtido para o modelo rígido e polarizado.

\section{Blindagem magnética}

Na análise seguinte, separamos as diferentes contribuições e analisamos primeiro a parte eletrostática. Os resultados obtidos com os modelos eletrostáticos PCM e ASEC estão dispostos na tabela 6.3. Para comparação com o experimento, consideramos os resultados para a blindagem magnética $\sigma\left({ }^{15} \mathrm{~N}\right)$ em piridina solvatada obtidos por Duthaler e Roberts [66]. É reportada em água, uma blindagem magnética de $-59,9 \mathrm{ppm}$. O valor corrigido para a susceptibilidade magnética de bulk é $-56,3 \mathrm{ppm}$. O modelo contínuo polarizado estima uma blindagem de $-81,0 \mathrm{ppm}$, o qual difere do experimento por 24,3 ppm.

Discutiremos agora os efeitos da polarização sobre $\sigma\left({ }^{15} \mathrm{~N}\right)$. Os resultados obtidos com o uso a geometria fixa são mostrados na figura 6.5. Observa-se que o resultado teórico caminha assintoticamente até a convergência em $-59,8$ ppm, em muito boa concordância com o valor experimental de $-59,9 \mathrm{ppm}$ ( ou $-56,3 \mathrm{ppm}$ se corrigido para a susceptibilidade magnética do bulk). Isso mostra que os efeitos da polarização têm grande influência sobre as blindagens magnéticas nucleares do nitrogênio em piridina. Combinando efeitos de polarização e geométricos, obtivemos um valor teórico de $-58,7 \mathrm{ppm}$. Assim, o efeito geométrico é estimado em apenas 1,1 ppm. Esse efeito tem duas componentes diretas. 
Tabela 6.3: Efeitos da polarização do soluto e relaxação de geometria calculados sobre as constantes de blindagem magnética nuclear $\sigma\left({ }^{15} \mathrm{~N}\right)$. As constantes magnéticas $(\sigma / \mathrm{ppm})$ são calculadas em nível de aproximação B3LYP $/ 6-311+\mathrm{G}(d, p)$ enquanto as geometrias (rígida e relaxada) são obtidas com a aproximação MP2/aug-cc-pVTZ:

\begin{tabular}{|c|c|c|}
\hline & \multicolumn{2}{|c|}{$\bar{\sigma}\left({ }^{15} \mathrm{~N}\right)$ em água } \\
\hline & Rígido & Relaxado \\
\hline Não-polarizado & $-82,1$ & $-78,0$ \\
\hline Polarizado & $-59,8$ & $-58,7$ \\
\hline $\mathrm{PCM}^{a}$ & $-81,0$ & - \\
\hline 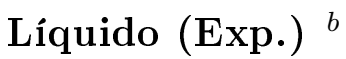 & - & $-56,3$ \\
\hline
\end{tabular}

(a) O resultado PCM foi obtido usando a geometria calculada para o gás em nível de aproximação MP2/aug-cc-pVTZ. (b) Diluído em ciclohexano [66]. Valor corrigido com relação à susceptibilidade magnética de bulk e extrapolado com relação à diluição infinita.

Uma, deve-se diretamente à modificações geométricas que afetam a blindagem magnética. A outra surge da redistribuição de cargas sobre o soluto, que modifica seu momento de dipolo, e altera o número de coordenação do solvente. O efeito combinado dessas duas componentes é muito pequeno. Como discutido, espera-se que as variações na geometria sejam pequenas. De fato, observamos pequenas variações de $0,006 \AA$ nas ligações $\mathrm{N}_{1}-\mathrm{C}_{2}$ e pequenos desvios de $0,1^{\circ}$ no ângulo $A\left(C_{2} N_{1} C_{6}\right)$. Contreras e Peralta [154] mostraram, em amônia, que a maior contribuição para o acoplamento indireto entre spins nucleares é oriunda de variações angulares. De modo geral, os nossos cálculos mostram que efeitos geométricos sobre a blindagem magnética $\sigma\left({ }^{15} \mathrm{~N}\right)$ em piridina são muito suaves, como notado anteriormente para sistemas similares [151].

Após analisarmos as contribuições eletrostáticas sobre a blindagem magnética, discutiremos agora as contribuições provenientes do solvente explícito ao redor do soluto. Para assegurar uma análise sistemática, consideraremos inicialmente o papel das ligações de hidrogênio (LH). Os resultados estão dispostos na tabela 6.4. Como os resultados discutidos acima mostram que efeitos geométricos são irrelevantes, todos os resultados apresentados nesta seção foram executados sobre as configurações Monte Carlo obtidas para a geometria rígida da piridina. A blindagem magnética sobre o átomo de nitrogênio, obtida com o uso somente de moléculas de água explícitas, formando LH com o soluto é $-83,4 \pm 0,7$ ppm. Resultado que difere $\sim 27$ ppm do experimento. Esses resultados são obtidos sobre estruturas que envolvem diferentes quantidades de moléculas de água por configuração (veja tabela 6.2). Esse modelo pode ser melhorado imergindo essas configurações no campo eletrostático das demais moléculas de água, representadas somente por cargas pontuais. Esse novo modelo é referenciado aqui como $\mathrm{LH}+\mathrm{CP}$, e essa situação é ilustrada na figura 6.4 . 


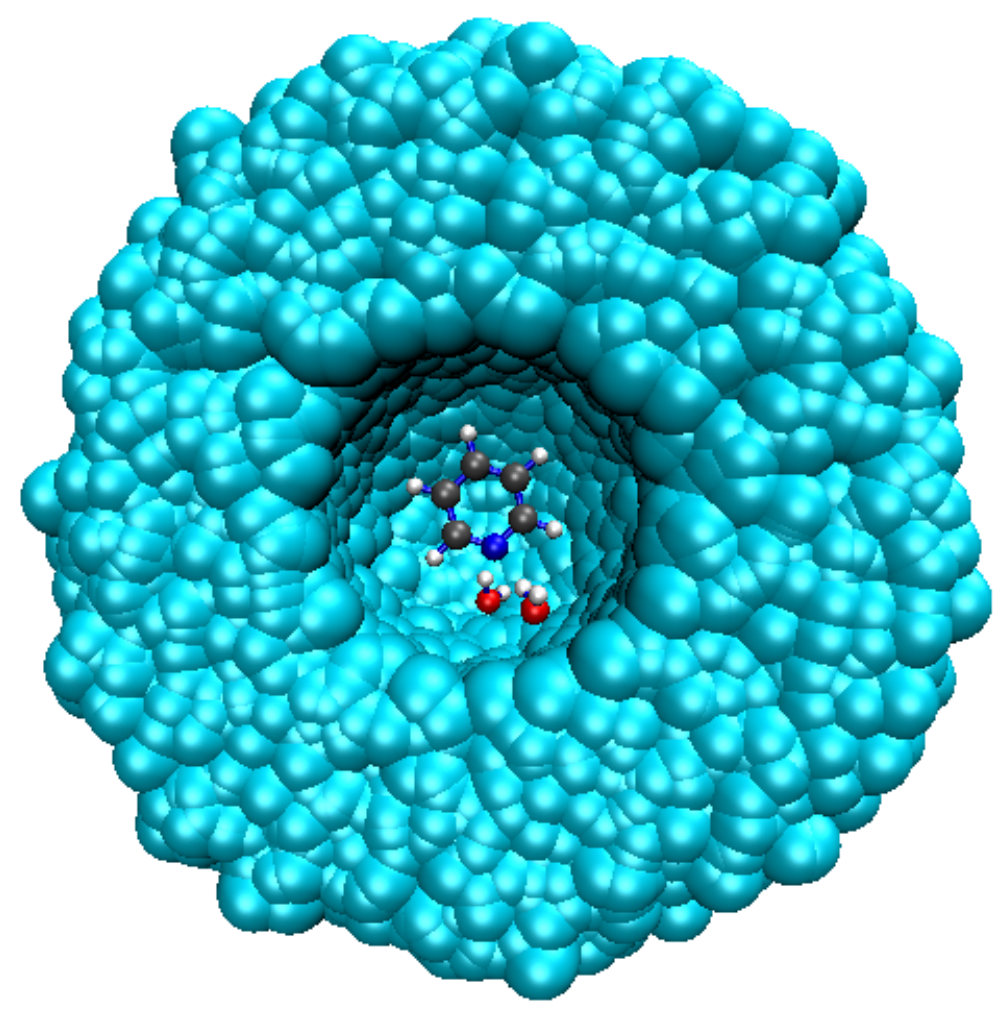

Figura 6.4: Estrutura piridina-água embebida no campo eletrostático das moléculas de água remanescentes.

As moléculas que integram o banho eletrostático são representadas apenas pelas cargas elétricas localizadas em suas respectivas posições atômicas, descritas pelo potencial clássico TIP3P usado nas simulações Monte Carlo. Em cada configuração LH+PC, 320 moléculas de água foram incluídas como cargas pontuais. A blindagem magnética calculada usando esse modelo é agora $-62,4 \pm 0,7$ ppm, em razoável concordância com o valor experimental de $-56,3 \mathrm{ppm}$. Esse resultado difere da predição iterativa ASEC por apenas $\sim 3$ ppm. Ao aumentarmos o número de moléculas explícitas incluídas no banho eletrostático (modelo $6 \mathrm{H}_{2} \mathrm{O}+\mathrm{CP}$ ), obtemos uma blindagem de $-61,8 \pm 0,7$ ppm, que embora seja uma predição mais próxima de experimento, difere por apenas 0,6 ppm do modelo $\mathrm{LH}+\mathrm{CP}$. Esse resultado mostra que a principal contribuição provém da formação de pontes de hidrogênio, bem como do campo elétrico das moléculas mais afastadas. Essa constatação está de acordo com observações anteriores [37], inclusive sobre diazinas [155], nas quais concluiu-se que poucas moléculas explícitas do solvente são necessárias, mas as contribuições mais importantes derivam de interações eletrostáticas de longo alcance. 
Tabela 6.4: Blindagens magnéticas $(\sigma / \mathrm{ppm})$ calculadas em nível de aproximação B3LYP/augcc-pVTZ para o ${ }^{15} \mathrm{~N}$ em piridina hidratada:

\begin{tabular}{cccccc}
\hline \hline \multicolumn{5}{c}{$\boldsymbol{\sigma}\left({ }^{\mathbf{1 5}} \mathbf{N}\right)$ em água } \\
\hline \hline PCM $^{a}$ & ASEC $^{b}$ & LH $^{c}$ & LH+CP $^{d}$ & $\mathbf{6 H}_{2} \mathbf{O}+\mathbf{P C}^{e}$ & Exp. $^{f}$ \\
\hline$-81,0$ & $-59,8(-58,7)$ & $-83,4 \pm 0,7$ & $-62,4 \pm 0,7$ & $-61,8 \pm 0,9$ & $-56,3[-59,9]$ \\
\hline \hline
\end{tabular}

(a) O resultado PCM foi obtido usando a geometria calculada para o gás em nível de aproximação MP2/aug-cc-pVTZ. (b) Os valores em parêntesis foram obtidos em água usando a geometria relaxada. (c) LH inclui somente estruturas piridina-água, formando ligações de hidrogênio (veja o texto). (d) LH $+\mathrm{PC}$ inclui estruturas piridina-água formando ligações de hidrogênio e 320 águas remanescentes tratadas como cargas pontuais. Consulte o texto. (e) $6 \mathrm{H}_{2} \mathrm{O}+\mathrm{PC}$ inclui além do soluto as 6 moléculas de água mais próximas de seu nitrogênio e embebidas no campo eletrostático das 320 moléculas de águas remanescentes tratadas como cargas pontuais. Consulte o texto. $(f)$ Valor corrigido [não corrigido] com relação à susceptibilidade magnética do bulk e extrapolado com relação à diluição infinita [66].

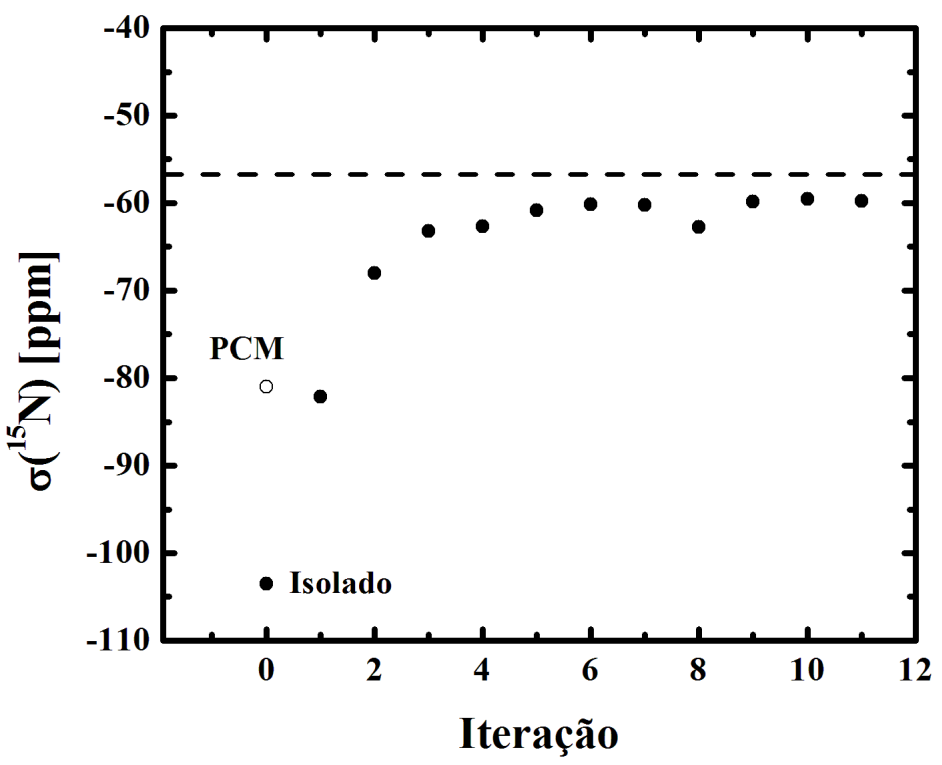

Figura 6.5: Evolução da blindagem magnética $\sigma\left({ }^{15} \mathrm{~N}\right)$ calculada como uma função dos passos iterativos. Os valores foram obtidos considerando o modelo rígido. O dado experimental é representado pela linha pontilhada.

Finalmente, discutiremos a influência da polarização do soluto sobre as cargas atômicas no nitrogênio. A figura 6.6 mostra que a polarização tem um efeito evidente sobre a redistribuição de cargas do soluto e que tal distribuição está intimamente relacionada com $\sigma\left({ }^{15} \mathrm{~N}\right)$. A figura 6.6 mostra que essa relação é linear e correlaciona o aumento da 
blindagem magnética com o aumento da densidade eletrônica no sítio aceitador de ligações de hidrogênio.

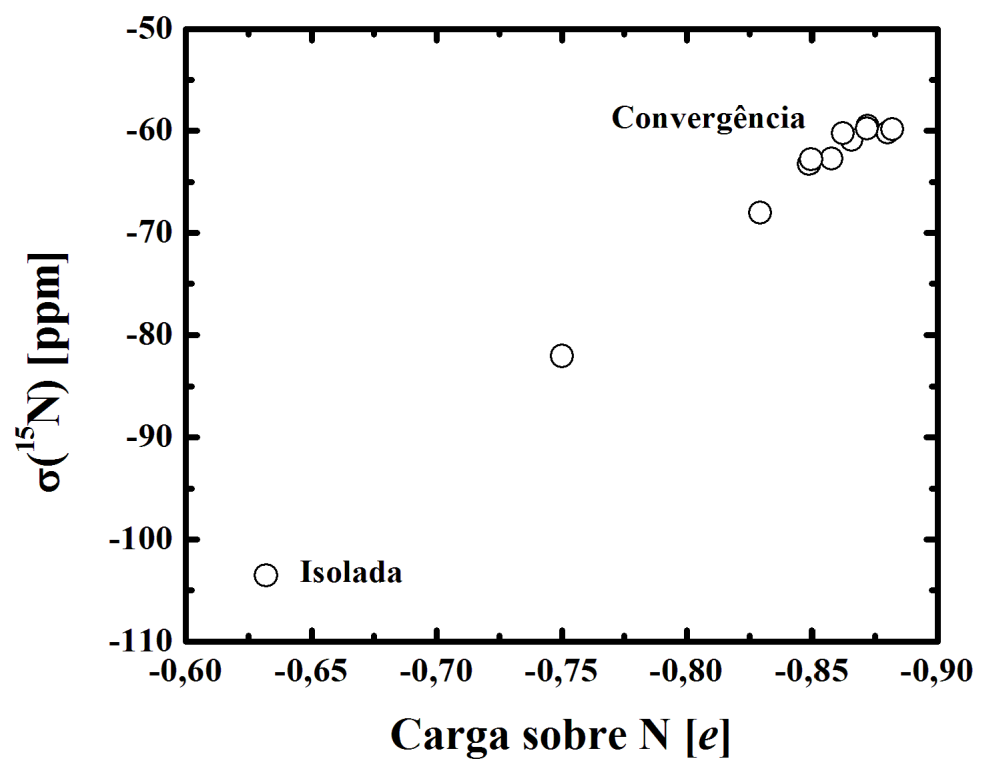

Figura 6.6: Blindagem magnética sobre o nitrogênio, calculada em cada passo do ciclo iterativo em função da população eletrônica sobre sítio atômico.

\subsection{Conclusões}

Analisamos a polarização eletrônica do soluto induzida pela interação com o solvente e suas implicações sobre a blindagem magnética $\sigma\left({ }^{15} \mathrm{~N}\right)$ em piridina. Para a molécula de piridina isolada calculamos (MP2/aug-cc-pVTZ) um momento de dipolo de 2,33 D, o qual está muito próximo do valor experimental de 2,15 \pm , 05 D. Em solução aquosa obtivemos valores variando entre 3,41 e 3,38 D, mostrando considerável efeito de polarização. A influência da polarização eletrônica sobre $\sigma\left({ }^{15} \mathrm{~N}\right)$ é então aferida. Mostramos que a polarização eletrônica exerce grande influência sobre o número de formação de ligações de hidrogênio e sobre as cargas atômicas do soluto. Com o uso de um procedimento iterativo, equilibramos as cargas do soluto na presença do solvente e constatamos a convergência da blindagem $\sigma\left({ }^{15} \mathrm{~N}\right)$ em piridina para o valor de $-59,9 \mathrm{ppm}$, em muito boa concordância com o valor experimental (-56,3 ppm). As variações sobre $\sigma\left({ }^{15} \mathrm{~N}\right)$ estão correlacionadas com as variações de cargas no nitrogênio da piridina por uma relação linear. Isso implica em uma redistribuição de cargas sobre o soluto devido a presença do solvente.

Os melhores resultados correspondem à piridina eletronicamente polarizada interagindo com as seis águas mais próximas, com todo o sistema embebido no banho eletrostático das águas remanescentes. Com essa aproximação, obtivemos uma blindagem de $-61,8$ ppm 
sobre o nitrogênio da piridina em boa concordância com os valores experimentais de $-56,3$ e -59,9 ppm. O shift gás-líquido está superestimado e atribuímos essa má descrição às incertezas encontradas nos resultados em vácuo. 


\section{Capítulo 7}

\section{Pirimidina hidratada}

\subsection{Introdução}

${ }^{1} \mathrm{H}$ e ${ }^{13} \mathrm{C}$ são os elementos mais comuns em compostos orgânicos, mas o ${ }^{15} \mathrm{~N}$ e ${ }^{17} \mathrm{O}$ mostram maior sensibilidade a interações com o meio. Os efeitos do solvente sobre as constantes magnéticas nucleares do ${ }^{15} \mathrm{~N}$, por exemplo, têm sido sistematicamente explorados por Witanowski e colaboradores [9, 10, 11, 12, 13, 14, 15] em vários sistemas nitrogenados, nos quais desvios gás-líquido de até 30 ppm têm sido observados. Contudo, investigações experimentais são ineficazes em separar e quantificar as contribuições de interações específicas, como ligações de hidrogênio. Esse tipo de interação tem reconhecido efeito sobre o espectro RMN, tendo motivado inúmeros trabalhos teóricos [36, 156, 157, 158, 159, 160, 161, 162, 163, 164, 165].

Pirimidina é um composto aromático heterocíclico similar ao benzeno e piridina (veja figura 7.1). Hábil em formar ligações de hidrogênio com o meio, pirimidina possui ainda inúmeras aplicações biológicas e industriais. A síntese de seus derivados tem sido exaustivamente discutida [166, 167, 168, 169, 170, 171], na qual aplicações para transferência de prótons [172], efeitos substitucionais [173] e, principalmente, a funcionalização de novos fármacos [174, 175] têm sido explorados. Em todas essas aplicações, RMN têm fornecido informações cruciais à caracterização estrutural desses compostos.

Neste capítulo utilizamos o tratamento s-QM/MM para estudar a dependência da blindagem magnética $\sigma\left({ }^{15} \mathrm{~N}\right)$ em piridina com relação ao solvente. Dispensamos atenção especial aos efeitos da polarização eletrônica induzida pelo meio. Para tanto, utilizamos modelos contínuos e discretos de solvente. As contribuições do solvente explícito também foram sistematicamente consideradas, primeiro incluindo nas aproximações quânticas as ligações de hidrogênio formadas entre pirimidina e água e, mais tarde, considerando uma maior quantidade de moléculas explícitas, até a convergência da propriedade de interesse. 


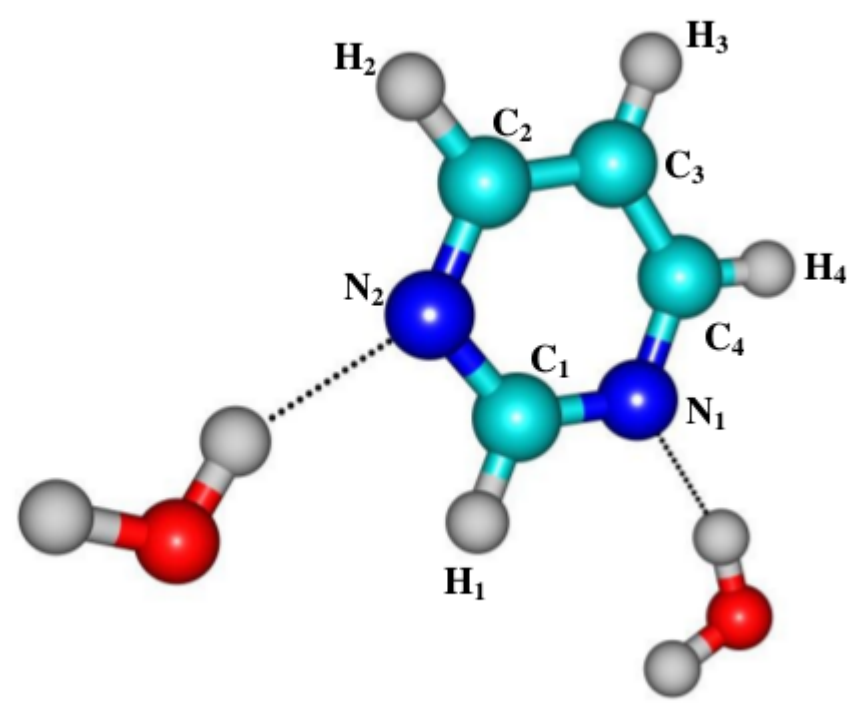

Figura 7.1: Pirimidina formando ligações de hidrogênio com duas moléculas de água. Os índices atômicos são usados para definir os parâmetros eletrostáticos na tabela 7.1.

\subsection{Detalhes computacionais}

Utilizamos a metodologia sequencial Monte Carlo/Mecânica Quântica [150, 176, 177, 178, 46] para calcular as propriedades magnéticas em pirimidina hidratada. Como em estudos anteriores, primeiro geramos as configurações do líquido executando simulações clássicas Monte Carlo (MC). Em seguida, executamos cálculos de mecânica quântica sobre essas configurações, tratadas como supermoléculas, compostas por uma molécula de piridina ladeada por várias moléculas de água.

As simulações foram executadas com o programa DICE [101] no ensemble NPT, usando o método das imagens e condições periódicas de contorno [76]. Simulamos o líquido à temperatura de $298 \mathrm{~K}$ e pressão de $1 \mathrm{~atm}$. O sistema é composto por uma molécula de pirimidina diluída em 1000 moléculas de água. A geometria do soluto foi obtida em nível de otimização MP2 $/ 6-31+\mathrm{G}(d, p)$. As interações intermoleculares foram modeladas pelos potenciais de Lennard-Jones (LJ) e Coulomb, com três parâmetros para descrever cada sítio atômico. Para a água, usamos o potencial SPC [181]. Para pirimidina usamos os parâmetros do campo de forças OPLS [182, 183] e as cargas nos sítios atômicos extraídas usando diferentes modelos de polarização eletrônica. Discutiremos esses procedimentos separadamente na seção 7.3. Nossas simulações foram divididas em duas partes. Em um estágio de termalização, um total de 2,1 × $10^{7}$ passos MC foram executados. Em 
seguida, em um estágio de produção, administramos mais $5 \times 10^{8}$ passos MC para gerar as configurações do líquido. Ao término desse processo, configurações estatisticamente correlacionadas em apenas $12 \%$ foram amostradas para subsequente tratamento com MQ.

As propriedades magnéticas foram discutidas em termos da teoria do funcional da densidade (DFT), usando o funcional B3LYP [145, 146]e os conjuntos de funções base aug-pcS- $n$ e pcS- $n$ [114], desenvolvidas especificamente para o estudo da blindagem magnética nuclear. Para diminuir o custo computacional, na supermolécula soluto-solvente, combinamos essas funções compondo a base aug-pcS-2 ${ }^{(\mathrm{N})} / \mathrm{pcS}-2^{(\mathrm{C}, \mathrm{O}, \mathrm{H})}$, onde os índices superiores indicam o elemento do soluto/solvente descrito pela respectiva base. Assim, os parâmetros RMN foram calculados usando o modelo B3LYP/aug-pcS-2 ${ }^{(\mathrm{N})} / \mathrm{pcS}-2^{(\mathrm{C}, \mathrm{O}, \mathrm{H})}$.

Modelos contínuos e discretos de solvente foram usados para extrair as propriedades RMN de interesse. Os modelos contínuos são representados pelo modelo polarizado PCM [25]. No modelo discreto, o solvente é representado somente pelo banho eletrostático (solvente representado por cargas pontuais), assim ao invés de executar cálculos de MQ em cada uma das 100 configurações MC, utilizamos uma configuração eletrostática média (ASEC) do solvente e somente executamos somente um cálculo de MQ. O procedimento ASEC tem origens na ideia do potencial eletrostático médio do solvente (ASEP - average solvent electrostatic potential) desenvolvido por Sánchez e colaboradores [184, 185, 186].

Todos os cálculos PCM foram administrados dentro do formalismo IEF (integral equation formalism) [187, 188]. Todas as constantes RMN foram obtidas usando o método GIAO (gauge-including atomic orbitals) [84, 116, 115] e todos os cálculos de mecânica quântica foram executados usando o programa Gaussian 03 [118].

\subsection{Resultados e discussões}

\section{Polarização eletrônica em água}

Discutiremos agora a inclusão dos efeitos de polarização. O campo de forças OPLS original da pirimidina $[182,183]$ é parametrizado para reproduzir as propriedades termodinâmicas do líquido homogêneo. Isto inclui cargas atômicas obtidas em nível HF/6-31G(d) usando o mapeamento eletrostático CHELPG [103]. Embora os parâmetros LJ tenham sido mantidos, as cargas atômicas foram adaptadas às interações pirimidina-água. A polarização do soluto adotada aqui ajusta as cargas eletrônicas ao solvente específico utilizado. Dois procedimentos são usados e os correspondentes momentos de dipolo serão comparados. Os procedimentos PCM (PCM-QM/MM) e iterativo (I-QM/MM) são então usados para calcular as cargas atômicas da molécula do soluto na presença do solvente. No procedimento I-QM/MM primeiro executamos um cálculo MP2/aug-cc-pVDZ da molécula isolada para obter as cargas atômicas usando o ajuste eletrostático CHELPG [103]. Essas cargas são 
inicialmente implementadas no potencial clássico das simulações MC. Então, configurações MC estatisticamente descorrelacionadas são amostradas para compor uma configuração eletrostática media do solvente (ASEC - average solvent eletrostatic configuration). Essa configuração ASEC é então utilizada para calcular em nível MP2/aug-cc-pVDZ a nova distribuição de cargas do soluto a serem implementadas em uma nova simulação MC. O processo é repetido iterativamente até a convergência do momento de dipolo. Nesse momento, as cargas eletrônicas do soluto estão em equilíbrio com o solvente. Os resultados estão resumidos na figura 7.2, na qual é mostrado o momento de dipolo convergido em água da pirimidina, obtido após poucas iterações. Exceto pelo primeiro ponto (iteração 0 ), todos os pontos na figura correspondem a um valor estatisticamente convergido do momento de dipolo em água. Cada configuração ASEC (cada ponto no gráfico) foi gerada com 100 configurações MC, estatisticamente descorrelacionadas, cada uma representando 500 moléculas de água como cargas pontuais. Isso inclui todas as moléculas do solvente dentro da distância de $15,3 \AA$ do centro de massa do soluto.

O momento de dipolo da pirimidina no modelo OPLS all-atoms original é 2, 52 D [182] enquanto que o resultado united-atoms é 2, 16 D [183]. Nosso resultado em vácuo (iteração 0 ), mostra que o momento de dipolo calculado da pirimidina é 2, 42 D. Esse resultado pode ser comparado à observação de 2,33 D obtida em gás [148]. O valor convergido em água é 4,08 D, correspondendo ao aumento de aproximadamente $70 \%$ relativo ao cálculo em vácuo. Os valores em água discutidos aqui são maiores que o valor teórico de 2, 93 D [189] obtido com uma metodologia mais simples. Para comparação, o correspondente em água obtido com o modelo PCM é 3, 50 D. Essa predição razoável origina outra alternativa que pode representar uma economia no tempo de computação. A saber, implementam-se as cargas PCM diretamente numa única simulação MC.

Na polarização PCM as cargas atômicas são usadas nas simulações MC para gerar as configurações soluto-solvente. O procedimento iterativo não é usado novamente a fim de equilibrar as cargas do soluto com o solvente, mas um novo ajuste eletrostático CHELPG, executado em nível de cálculo MP2/aug-cc-pVDZ, reproduz um momento de dipolo de 3, 89 D. Todos esses resultados são mostrados na figura 7.2. As propriedades magnéticas em pirimidina hidratada serão obtidas mediante esses dois esquemas de polarização. As cargas atômicas geradas, usando esses dois procedimentos, estão dispostas na tabela 7.1.

Resultados experimentais em solução, para o momento de dipolo, não são facilmente obtidos. Apenas resultados indiretos podem ser obtidos. Uma das possibilidades consiste em usar as intensidades do espectro infravermelho [190]. Para pirimidina hidratada, aparentemente não há resultados experimentais. 
Tabela 7.1: Parâmetros Lennard-Jones e Coulomb para pirimidina usados nas simulações Monte Carlo. Também são mostrados os momentos de dipolo (D) e cargas (e) calculados (MP2/augcc-PVDZ) em água.

\begin{tabular}{crrcc}
\hline \hline Sítio & $\boldsymbol{q}_{\mathbf{P C M}}$ & $\boldsymbol{q}_{\text {iterativo }}$ & $\boldsymbol{\varepsilon}_{\boldsymbol{i}}(\mathrm{kcal} / \mathbf{m o l})$ & $\boldsymbol{\sigma}_{\boldsymbol{i}}(\AA)$ \\
\hline \hline $\mathbf{C}_{\mathbf{4}} \mathbf{N}_{\mathbf{2}} \mathbf{H}_{\mathbf{4}}$ & & & & \\
$\mathbf{N}_{\mathbf{1}}$ & $-0,868$ & $-0,977$ & 0,170 & 3,250 \\
$\mathbf{N}_{\mathbf{2}}$ & $-0,868$ & $-0,985$ & 0,170 & 3,250 \\
$\mathbf{C}_{\mathbf{1}}$ & 0,866 & 0,986 & 0,110 & 3,750 \\
$\mathbf{C}_{\mathbf{2}}$ & 0,640 & 0,712 & 0,110 & 3,750 \\
$\mathbf{C}_{\mathbf{3}}$ & $-0,599$ & $-0,618$ & 0,110 & 3,750 \\
$\mathbf{C}_{\mathbf{4}}$ & 0,640 & 0,720 & 0,110 & 3,750 \\
$\mathbf{H}_{\mathbf{1}}$ & $-0,039$ & $-0,059$ & 0,000 & 0,000 \\
$\mathbf{H}_{\mathbf{2}}$ & 0,007 & $-0,003$ & 0,000 & 0,000 \\
$\mathbf{H}_{\mathbf{3}}$ & 0,214 & 0,220 & 0,000 & 0,000 \\
$\mathbf{H}_{\mathbf{4}}$ & 0,007 & 0,004 & 0,000 & 0,000
\end{tabular}

Momento de dipolo

$\langle\boldsymbol{\mu}\rangle \quad 3,50^{a} 3,89^{b} \quad 4,08$

(a) Valor obtido com o modelo PCM. (b) Valor obtido com o modelo PCM-QM/MM (veja texto).

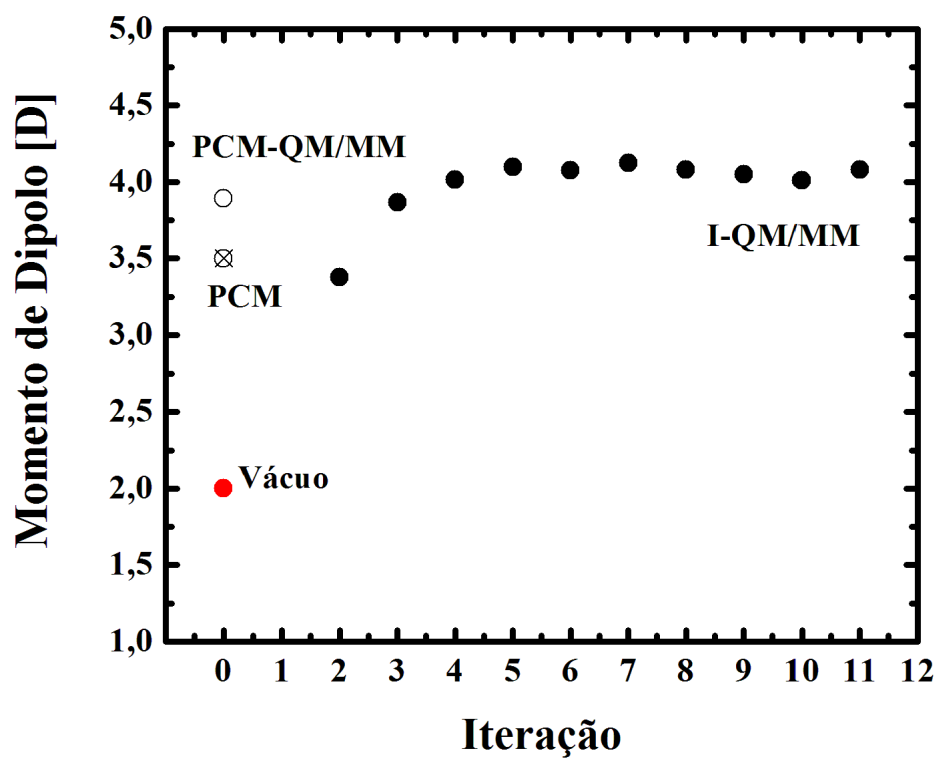

Figura 7.2: Valores calculados para o momento de dipolo da pirimidina hidratada usando o procedimento iterativo (I-QM/MM). Cada ponto é obtido com uma média envolvendo 100 configurações Monte Carlo. Também são mostrados os valores obtidos em vácuo (vermelho), PCM-QM/MM (círculo vazio) e PCM. 


\section{Aspectos estruturais e ligações de hidrogênio}

Um ponto importante em simulações computacionais de líquidos é a caracterização da estrutura do solvente ao redor da molécula do soluto. Isso implica na identificação estatística das camadas de solvatação e ligações de hidrogênio soluto-solvente. Uma maneira mais eficiente e correta de estudar a formação de ligações de hidrogênio entre soluto-solvente foi proposta previamente por Stillinger e Rahman [152], Mezei e Beveridge [153], Rapaport [191] e tem sido frequentemente utilizada [33, 176, 178, 188]. Consideramos a formação de uma ligação de hidrogênio quando os valores $R_{\mathrm{N}-\mathrm{O}} \leq 3,2 \AA, \theta_{\mathrm{N}-\mathrm{OH}} \leq 35^{\circ}$ e $E \leq-3,2$ $\mathrm{kcal} / \mathrm{mol}$ (na polarização $\mathrm{PCM}$ ) e $E \leq-3,7 \mathrm{kcal} / \mathrm{mol}$ (na polarização iterativa) são satisfeitos. Assim, a distância entre o átomo de hidrogênio da pirimidina e o oxigênio da

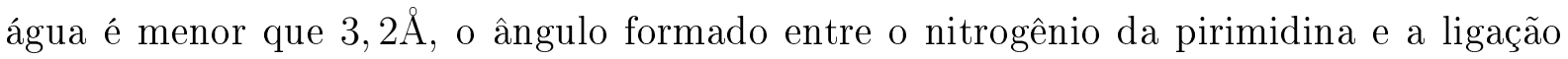
$\mathrm{OH}$ tem $35^{\circ}$ como limite superior e a energia de interação é pelo menos $-3,2 \mathrm{kcal} / \mathrm{mol}$ (PCM) ou $-3,7 \mathrm{kcal} / \mathrm{mol}$ (iterativo). Os critérios geométricos (distância e ângulo) são respectivamente extraídos das funções de distribuições radiais e angulares. Os critérios energéticos podem ser facilmente obtidos analisando a energia de interação de pares entre soluto e solvente. Usando esses parâmetros, obtivemos médias 2,1 e 2,4 ligações de hidrogênio nas polarizações PCM e iterativa, respectivamente.

Tabela 7.2: Estatística (\%) da formação de ligações de hidrogênio (LH) em pirimidina hidratada usando duas (PCM e iterativa) polarizações eletrônicas distintas:

\begin{tabular}{crr}
\hline \hline Número de LH & PCM & Iterativa \\
\hline \hline $\mathbf{1}$ & 14,1 & 9,1 \\
$\mathbf{2}$ & 63,6 & 43,4 \\
$\mathbf{3}$ & 21,2 & 41,4 \\
$\mathbf{4}$ & 1,1 & 6,1 \\
Média & 2,1 & 2,4 \\
\hline \hline
\end{tabular}

A estatística das ligações de hidrogênio pode ser discutida em detalhes junto à tabela 7.2. No caso da polarização PCM, por exemplo, encontramos em $14 \%$ das configurações a ocorrência de apenas uma ponte, $63,6 \%$ das configurações formando duas ligações, $21 \%$ com três ligações e apenas 1,1\% com quatro ligações. Os resultados para a polarização iterativa também estão dispostos na tabela 7.2. Interações soluto-solvente na forma de ligações de hidrogênio têm particular interesse em RMN. Nos cálculos de mecânica quântica subsequentes utilizaremos esses estruturas para analisar a dependência da blindagem magnética $\sigma\left({ }^{15} \mathrm{~N}\right)$ com relação à formação de ligações de hidrogênio pirimidina-água. 


\section{Efeito do solvente sobre a blindagem magnética $\sigma\left({ }^{15} \mathrm{~N}\right)$}

Pirimidina em água está sujeita à formação de ligações de hidrogênio, as quais são conhecidas por terem grande influência sobre a blindagem magnética nuclear $\sigma\left({ }^{15} \mathrm{~N}\right)$. Diazinas têm sido continuamente estudadas por Witanowski e colaboradores [9]. Não há resultados para o shift gás-líquido em piridina, mas o desvio ciclohexano-água tem sido reportado como 16,8 ppm. Esse efeito do solvente tem sido reportado em vários estudos teóricos realizados por Mennucci e colaboradores [155, 179]. Uma investigação recente usando o modelo PCM em nível de aproximação B3LYP $/ 6-311+\mathrm{G}(d, p)$ e combinada ao procedimento GIAO, indica efeitos de solvente de 6, 35 e 10, 35 ppm para os desvios ciclohexanoágua e gás-água, respectivamente. Apesar da predição limitada em gás e ciclohexano, uma boa correlação entre teoria e experimento tem sido observada para outros solventes [179]. Esses cálculos sugerem uma diferença gás-ciclohexano de 4,18ppm.

O uso explícito de algumas moléculas de solvente tem a vantagem de introduzir efeitos não eletrostáticos que não podem ser contemplados pelos modelos contínuos. Resultados melhores [155, 179] têm sido obtidos considerando poucas moléculas de água, mesmo em aglomerados otimizados.

O presente estudo considera nos cálculos de mecânica quântica todas as moléculas de água envolvidas em interações por ligações de hidrogênio, como discutido na seção anterior. Assim, além de interações explícitas, as condições termodinâmicas do líquido também são preservadas. Em outro momento, as moléculas mais afastadas do soluto serão incluídas e interações de longo alcance representadas pelo campo elétrico das moléculas remanescentes. Os principais resultados estão representados na tabela 7.3, na qual são consideradas duas polarizações eletrônicas do soluto. Estudos anteriores utilizando estes modelos [192] têm mostrado que a aproximação PCM tem uma tendência a subestimar o efeito do solvente em diazinas. Como exemplo, a predição PCM para o shift gáságua em pirimidina é apenas 12,2 ppm [192]. Essa descrição pobre tem sido atribuída ao tratamento inadequado, dado pelos modelos contínuos, à formação de ligações de hidrogênio pirimidina-água. Observamos que a inclusão de moléculas de água ligadas ao soluto por ligações de hidrogênio tem melhorado significativamente a concordância entre teoria e experimento. Por exemplo, usando a polarização PCM nas simulações MC, obtivemos 17,4 ppm para o desvio gás-água $\Delta \sigma\left({ }^{15} \mathrm{~N}\right)$, em boa concordância com o desvio experimental de 16,8 ppm obtido em ciclohexano-água. Para aprimorar o nível de aproximação, embebemos moléculas explícitas do solvente no banho eletrostático proveniente das moléculas de água remanescentes. Para tanto, consideramos dois procedimentos diferentes. Primeiro, incluímos no banho eletrostático apenas as moléculas de água formando ligações de hidrogênio com o soluto. Esse modelo é referenciado como $\mathrm{LH}+\mathrm{CP}$. No caso da polarização PCM, essa aproximação prediz $24,5 \pm 0,8$ ppm, superestimando $\Delta \sigma\left({ }^{15} \mathrm{~N}\right)$. 
Tabela 7.3: Desvio gás-água calculado sobre a componente isotrópica da blindagem magnética nuclear em pirimidina usando diferentes modelos de polarização e em nível de aproximação B3LYP/aug-pcS-2 ${ }^{(\mathrm{N})} / \mathrm{pcS}-2^{(\mathrm{C}, \mathrm{O}, \mathrm{H})}$. Para referência, a componente isotrópica da blindagem magnética calculada em vácuo é 91,7 ppm e desvio obtido com o modelo PCM é 15,4 ppm.

\begin{tabular}{ccccc}
\hline \hline Polarização & ASEC & LH & LH+CP & $\mathbf{9 H}_{\mathbf{2}} \mathbf{O}+\mathbf{C P}$ \\
\hline \hline PCM & 25,8 & $17,4 \pm 1,0$ & $24,5 \pm 0,8$ & $24,4 \pm 0,8$ \\
Iterativa & 30,4 & $16,4 \pm 0,7$ & $27,8 \pm 0,7$ & $28,5 \pm 0,8$ \\
\hline \hline
\end{tabular}

(a) O desvio experimental ciclohexano-água é 16,8 ppm. (b) Desvio calculado gás-ciclohexano é 4,2 ppm. (c) O desvio experimental sugerido é $17-21 \mathrm{ppm}$ (veja texto).

Como o resultado ASEC (25, 8 ppm) mostra, a contribuição eletrostática é muito grande, estando essa contribuição refletida também na predição LH $+\mathrm{CP}$. Um modelo mais realístico $\left(9 \mathrm{H}_{2} \mathrm{O}+\mathrm{CP}\right)$ é obtido considerando explicitamente no banho eletrostático algumas moléculas adicionais do solvente. Contudo, esse modelo não fornece diferenças significantes. Essa aproximação prediz $24,4 \pm 0,8 \mathrm{ppm}$, corroborando o resultado $\mathrm{LH}+\mathrm{CP}$. Concluímos que o uso explícito das ligações de hidrogênio em água é fundamental para a descrição da blindagem magnética nuclear em pirimidina.

Nosso melhor resultado aponta um efeito de solvente gás-água de 24, 4 pppm. Como discutido anteriormente, o valor experimental ciclohexano-água é 16,8 ppm. Portanto, a análise do desvio gás-ciclohexano, necessária para uma melhor comparação teoriaexperimento, é uma questão em aberto. Após considerar vinte diferentes solventes, a figura 7.3 mostra os resultados experimentais da referência [9] obtidos para o desvio químico (chemical shift) da pirimidina em função de escala de polarização proposta por Reichardt [193]. Podemos notar que a extrapolação ao gás $\left(\mathrm{E}_{\mathrm{T}}{ }^{\mathrm{N}}=-0,111\right)$ [193] é possível, embora com algumas incertezas. Essa extrapolação sugere um desvio gás-água de $18,5 \pm 2 \mathrm{ppm}$. Após adicionar esse valor à estimativa prévia de 4,18 ppm para o desvio gás-ciclohexano, obtemos um desvio gás-água de aproximadamente 21 ppm.

$\mathrm{Na}$ discussão acima, assumimos que desvio gás-água experimental para o ${ }^{15} \mathrm{~N}$ em pirimidina esteja de fato na faixa compreendida entre $17-21 \mathrm{ppm}$. Para a polarização PCM, obtemos assim uma boa concordância entre nosso melhor modelo $\left(9 \mathrm{H}_{2} \mathrm{O}+\mathrm{CP}\right)$ e experimento. Na polarização iterativa, os resultados superestimam em $7-8$ ppm nossa "predição" do experimento. Considerando as dificuldades em obter determinações experimentais precisas sobre a blindagem $\sigma\left({ }^{15} \mathrm{~N}\right)$, acreditamos que a predição iterativa forneça resultados muito bons.

Agora discutiremos brevemente algumas das possíveis fontes de inconsistências nos cálculos das constantes magnéticas nucleares em pirimidina. Como nossa geometria foi obtida em vácuo, discutiremos primeiramente como isso pode afetar a qualidade de nos- 
sos resultados. A geometria da piridina em água pode ser obtida em PCM ou SCRF ( self consistent reaction field), mas, devido à rigidez da molécula, as poucas diferenças geométricas afetariam irrelevantemente nossos resultados. Em piridina hidratada por exemplo, os efeitos de relaxação geométrica sobre $\sigma\left({ }^{15} \mathrm{~N}\right)$ são da ordem de 1, 1 ppm [194].

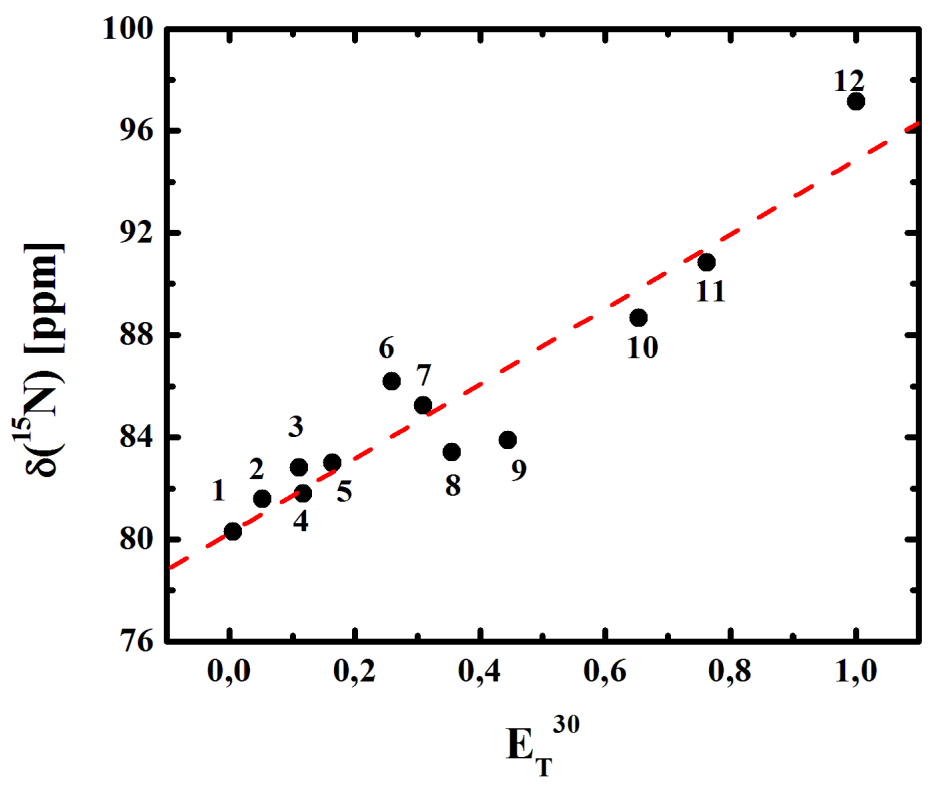

Figura 7.3: Dependência do desvio químico experimental da pirimidina em função da escala de polarização de Reichardt [193], normalizada para vinte solventes diferentes. Os valores numéricos para o desvio químico foram extraídos dos experimentos de Witanowski et al. [9], realizados em diferentes solventes: $1=$ ciclohexano, $2=\mathrm{CCl}_{4}, 3=$ benzeno, 4 $=\mathrm{Et}_{2} \mathrm{O}, 5=$ dioxano, $6=\mathrm{CHCl}_{3}, 7=\mathrm{CH}_{2} \mathrm{C}_{12}, 8=$ acetona, $9=\mathrm{DMSO}, 10=\mathrm{EtOH}$, $11=\mathrm{MeOH}, 12=\mathrm{H}_{2} \mathrm{O}$.

Outra clara fonte de incertezas tem origens na negligência de correções vibracionais de ponto-zero (ZPVC - zero-point vibrational corrections). Estudos anteriores [195, 105] mostram que ZPVC têm grande contribuição sobre constantes RMN, mas como os experimentos em gás, ciclohexano e em água foram realizados à temperatura ambiente, considerar esse tipo de correção nos cálculos de mecânica quântica produziria resultados não físicos.

Resta agora discutir o modelo teórico B3LYP/aug-pcS-2 ${ }^{(\mathrm{N})} / \mathrm{pcS}-2^{(\mathrm{C}, \mathrm{O}, \mathrm{H})}$. Poderíamos supor que o funcional B3LYP estaria superestimando as blindagens magnéticas, sendo uma das maiores causas de erros em nossa predição. Contudo, investigações teóricas para amônia gasosa [108] e líquida [139] têm comprovado a boa performance do funcional B3LYP em conjunto às bases aug-pcS- $n$. Ao adotar como referência a base aug-pcS-3, nossos testes mostraram erros de apenas 1,0 ppm para a base aug-pcS-2 ${ }^{(\mathrm{N})} / \mathrm{pcS}-2^{(\mathrm{C}, \mathrm{O}, \mathrm{H})}$. Assim, não podemos atribuir maiores deficiências à escolha da base. Contudo, tem sido re- 
centemente discutido que algumas dessas bases, desenvolvidas especificamente para estudo de propriedades magnéticas nucleares, podem reproduzir situações não físicas [151, 194]. Finalmente, a falta da polarização eletrônica do solvente devido ao soluto tem pequenos efeitos sobre $\sigma\left({ }^{15} \mathrm{~N}\right)[139,192]$. Trabalhos recentes têm mostrado que essas contribuições são mais importantes para o acoplamento indireto entre spins nucleares [139, 192].

\subsection{Conclusões}

Investigamos os efeitos do solvente sobre a blindagem magnética nuclear $\sigma\left({ }^{15} \mathrm{~N}\right)$ em pirimidina hidratada, usando o tratamento s-QM/MC. Como as parte clássicas e quânticas não são acopladas no tratamento sequencial é necessária uma consideração especial da polarização eletrônica. Isso foi feito através de duas aproximações diferentes. Primeiro utilizamos um método iterativo que equilibra as cargas do soluto na presença do solvente. A segunda alternativa reconcilia as aproximações PCM e QM/MM. Após obter a polarização eletrônica através do modelo PCM, empregamos as novas cargas na parte MM da metodologia s-QM/MM. Analisamos a polarização do soluto monitorando o momento de dipolo da pirimidina em água. Os valores calculados são 3,89 D (polarização PCM) e 4,08 D (polarização iterativa), correspondendo ao aumento de $61 \%$ e $69 \%$, relativo ao dipolo de 2,42 $\mathrm{D}$, calculado em vácuo.

Os resultados obtidos com as duas polarizações são utilizados para analisar os aspectos estruturais do líquido, bem como a formação de ligações de hidrogênio entre piridina e água. A polarização PCM prediz uma média de 2,1 ligações de hidrogênio por estrutura, valor ligeiramente menor que a média de 2,4 ligações obtidas na polarização iterativa.

Finalmente, discutimos a blindagem magnética nuclear $\sigma\left({ }^{15} \mathrm{~N}\right)$ e sua dependência com relação à polarização eletrônica do soluto devido ao meio, bem como, quanto à formação de ligações de hidrogênio soluto-solvente. Os melhores resultados foram obtidos para a piridina eletronicamente polarizada, rodeada por poucas moléculas explícitas de solvente, e todo o sistema embebido no campo eletrostático das águas remanescentes. Nesta aproximação utilizamos nove moléculas explícitas de água para obter desvios gás-água de 24,4 \pm 0, 8 ppm (polarização PCM) e 28,5 \pm 0, 8 ppm (polarização iterativa). Essas predições são comparáveis ao valor experimental inferido de $17-21 \mathrm{ppm}$. Uma análise detalhada mostrou que as contribuições mais relevantes para a blindagem $\sigma\left({ }^{15} \mathrm{~N}\right)$ provêm da formação de ligações de hidrogênio, mas uma descrição consistente do experimento somente é obtida após considerar as contribuições eletrostáticas das moléculas mais afastadas.

Ao considerar a simplicidade do modelo PCM-QM/MM comparado à polarização iterativa, a concordância obtida, comparando esses dois procedimentos, é um importante 
aspecto em se tratando de simulações computacionais e sugere uma alternativa economicamente mais viável no trato de sistemas moleculares de grande porte. 


\section{Capítulo 8}

\section{Amônia líquida}

\subsection{Introdução}

Apresentamos agora um estudo detalhado dos parâmetros RMN em amônia líquida. As propriedades de interesse foram obtidas usando modelos contínuos e explícitos de solvente. Usados com relativo sucesso, modelos contínuos têm origem nas ideias de Onsager [23] e Kirkwood [24]. Por outro lado, modelos explícitos de solvente representam uma descrição mais fiel da estrutura líquida, mas demandam maior esforço computacional. Em particular, utilizamos um procedimento sequencial QM/MM [196], no qual a estrutura do líquido é gerada através de simulações clássicas Monte Carlo (MC). Em seguida, a blindagem magnética $\sigma\left({ }^{15} \mathrm{~N}\right)$ e as constantes de acoplamento ${ }^{1} J(\mathrm{~N}-\mathrm{H})$ e ${ }^{2} J(\mathrm{H}-\mathrm{H})$ foram estudadas realizando cálculos de mecânica quântica sobre as configurações geradas nas simulações MC.

Com relação à blindagem magnética $\sigma\left({ }^{15} \mathrm{~N}\right)$, Litchman [197] e colaboradores foram os primeiros a reportar os valores de 264, 3 e 241, 7 ppm em amônia isolada e líquida, respectivamente. O shift gás-líquido de $-22,6 \pm 0,2$ ppm mostra claramente que a blindagem sobre o átomo de nitrogênio depende fortemente do meio.

Pertence a Bernheim e Berthier o primeiro trabalho experimental sobre o acoplamento intramolecular em amônia líquida [198]. Para o acoplamento ${ }^{1} J(\mathrm{~N}-\mathrm{H})$, Bernheim e Berthier reportam um valor de $-61,2 \pm 0,9 \mathrm{~Hz}$. No caso da amônia, os três hidrogênios são equivalentes, com isso, o acoplamento ${ }^{2} J(\mathrm{H}-\mathrm{H})$ não pode ser medido diretamente. Bernheim e Berthier obtiveram um valor de $-10,35 \pm 0,80 \mathrm{~Hz}$ através da relação ${ }^{2} J(\mathrm{H}-\mathrm{H})$ $=\left(\gamma_{H} / \gamma_{D}\right)^{2} J(\mathrm{D}-\mathrm{H})$, partindo de amônia deuterada, $\mathrm{NH}_{2} \mathrm{D}$. Estudos mais recentes, como os de Wasilyshen e Friendrich [199], determinaram os valores de $-61,45 \pm 1,3$ e $-9,6 \pm 1,3$ $\mathrm{Hz}$ para os acoplamentos ${ }^{1} J(\mathrm{~N}-\mathrm{H})$ e ${ }^{2} J(\mathrm{H}-\mathrm{H})$, respectivamente. Finalmente, Jameson e colaboradores [200, 201] observaram, nesta ordem, valores de $-61,7 \pm 0,2$ e $-61,8 \pm 0,5$ $\mathrm{Hz}$ para os acoplamentos ${ }^{1} \mathrm{~J}(\mathrm{~N}-\mathrm{H})$ em amônia isolada e líquida. Embora não haja resulta- 
dos para o acoplamento ${ }^{2} J(\mathrm{H}-\mathrm{H})$ em amônia isolada, os dados vigentes concordam e são bastante precisos com relação ao líquido.

Está claro que constantes magnéticas nucleares em amônia têm sido bastante estudadas e mapeadas, mas há dúvidas frequêntes sobre quais fatores têm efeito preponderante sobre essas propriedades moleculares. Por exemplo, Mennucci e colaboradores usaram modelos contínuos no trato de sistemas moleculares nitrogenados [202]. Conclui-se, como regra geral, que o solvente precisa ser incluído explicitamente nos cálculos de mecânica quântica, a fim de garantir uma descrição consistente da blindagem magnética. Contudo, para um série de sistemas, tem-se discutido que contribuições eletrostáticas e a polarização eletrônica do soluto são os fatores primordiais à descrição de constantes magnéticas nucleares [36, 139, 194]. A riqueza de informações experimentais sobre as propriedades magnéticas em amônia a torna um excelente laboratório para o desenvolvimento de novas metodologias, ajudando a responder perguntas sobre o efeito do solvente sobre propriedades magnéticas nucleares. As contribuições provenientes da polarização do soluto e relaxação de geometria causados pelas interações com o meio foram sistematicamente consideradas neste estudo. Modelos contínuos, discretos e explícitos foram usados na descrição do solvente. Como resultado foi possível determinar quais contribuições são importantes na descrição dos acoplamentos nucleares ${ }^{1} J(\mathrm{~N}-\mathrm{H})$ e ${ }^{2} J(\mathrm{H}-\mathrm{H})$, bem como à blindagem magnética $\sigma\left({ }^{15} \mathrm{~N}\right)$.

Um ponto crucial e sistematicamente atacado por Witanowski e colaboradores, concerne sobre os efeitos da redistribuição de cargas eletrônicas no soluto devido à polaridade do solvente $[9,10,11,12,13,14,15]$. Tem sido relacionado o aumento da blindagem eletrônica sobre ${ }^{15} \mathrm{~N}$ e ${ }^{17} \mathrm{O}$ com o aumento da população eletrônica sobre esses isótopos. Em recentes trabalhos $[45,121,194]$, temos mostrado que essa relação é essencialmente verdadeira para sistemas em que esses isótopos comportam-se como aceitadores de ligações de hidrogênio. Discutiremos, começando por agora, situações particulares em que as observações feitas por Witanowski e colaboradores falham.

\subsection{Detalhes computacionais}

No tratamento sequencial Monte Carlo / Mecânica Quântica, as configurações do líquido são geradas por simulações clássicas MC, em seguida as propriedades moleculares de interesse são calculadas tratando essas configurações com aproximações quânticas [196]. Simulações computacionais geram um número excessivo de estruturas, mas apenas um número de configurações estatisticamente descorrelacionadas é suficiente para obter as propriedades moleculares médias de interesse [203]. Essas configurações representativas são amostradas por meio da uma função de auto-correlação de energias do sistema $C(i)$. 
No caso de líquidos, essa função é comumente representada pelo decaimento exponencial duplo

$$
C(i)=a_{1} e^{-i / \tau_{1}}+a_{2} e^{-i / \tau_{2}}
$$

onde $\tau=\tau_{2}+\tau_{2}$ determina a correlação estatística. As configurações são selecionadas para intervalos suficientemente grandes $(\sim 2 \tau)$. Desse modo, são obtidas estruturas correlacionadas em apenas $13 \%$.

Todas as simulações clássicas MC foram desenvolvidas com o programa DICE [101], no ensemble NPT, com 500 moléculas de amônia. Todas as interações intermoleculares foram asseguradas através dos potenciais de Lennard-Jones (LJ) e Coulomb

$$
U\left(r_{i j}\right)=\sum_{i j}\left\{4 \varepsilon_{i j}\left[\left(\frac{\sigma_{i j}}{r_{i j}}\right)^{12}-\left(\frac{\sigma_{i j}}{r_{i j}}\right)^{6}\right]+\frac{q_{i} q_{j} e^{2}}{4 \pi \epsilon r_{i j}}\right\}
$$

com três parâmetros para cada sítio. Testamos as performances dos campos de forças desenvolvidos por Impey e Klein (IK) [204] e Gao, Xia e George (GXA) [205]. Somente os parâmetros LJ foram extraídos dos potenciais IK e GXG. As cargas eletrônicas dos sítios atômicos foram obtidas através do procedimento iterativo [47, 48] descrito nos capítulos precedentes. O procedimento iterativo permite atualizar as cargas do soluto na presença do solvente, incluindo os efeitos da polarização eletrônica do soluto devido ao solvente. Contudo, em se tratando de líquidos homogêneos, é possível discutir a polarização recíproca do solvente em devida ao soluto. Isso pode ser alcançado atualizando simultaneamente nas simulações MC as cargas eletrônicas do soluto e solvente. Novamente as cargas eletrônicas foram calculadas (MP2/aug-cc-pVTZ) usando o ajuste eletrostático CHELPG [103], que ajusta o potencial clássico ao quântico.

Em piridina, mostramos que efeitos de relaxação geométrica sobre a blindagem magnética nuclear $\sigma\left({ }^{15} \mathrm{~N}\right)$ são irrelevantes. Contudo, o acoplamento indireto entre spins nucleares é extremamente sensível a variações estruturais. Em amônia líquida, discutiremos novamente essas contribuições, tendo como foco os acoplamentos ${ }^{1} J(\mathrm{~N}-\mathrm{H})$ e e $J(\mathrm{H}-\mathrm{H})$. Discutiremos esses efeitos através dos modelos fixos e relaxados de soluto. Nos modelos fixos, somente as cargas eletrônicas do soluto são relaxadas durante o processo iterativo. Nos modelos relaxados, tanto geometria quanto cargas do soluto são relaxadas na presença do solvente. Os modelos relaxados são baseados no método do gradiente de energia livre (FEG - free energy gradient) desenvolvido por Nagaoka e colaboradores [130, 131, 132] e recentemente usado por Aguilar [140,141] para obter estruturas moleculares em ambiente líquido.

Bons resultados têm sido obtidos no estudo de constantes magnéticas nucleares usando a teoria do funcional da densidade $[45,104,105,108,121]$. Devido a boa descrição 
obtida para sistemas nitrogenados [45, 105, 108, 121], optamos por estudar as constantes RMN em amônia líquida usando o funcional semi-empírico B3LYP [145, 146]. Optamos por descrever a blindagem magnética nuclear $\sigma\left({ }^{15} \mathrm{~N}\right)$ através das bases aug-pcJ-3 [114], testadas em amônia isolada, e desenvolvidas para o estudo de blindagems magnéticas nucleares. Os acoplamentos intramoleculares ${ }^{1} J(\mathrm{~N}-\mathrm{H})$ e ${ }^{2} J(\mathrm{H}-\mathrm{H})$ também são discutidos em termos das bases apropriadas aug-pcJ-2 [206] e aug-cc-pVTZ-J [67]. Todos as cálculos de mecânica quântica foram executados dentro do formalismo GIAO [84, 115, 116] como implementado no programa Gaussian 03 [118].

\subsection{Resultados e discussões}

\section{Polarização do soluto}

A tabela 8.1 mostra os resultados calculados (MP2/aug-ccpVTZ) para o momento de dipolo em amônia líquida. O dipolo calculado para a molécula isolada é 1,562 D, em excelente concordância com o valor experimental 1,56 D [207]. O efeito da polarização sobre o soluto se reflete em um sensível aumento no momento de dipolo. Dispensamos especial atenção sobre os efeitos da polarização eletrônica do soluto. No modelo de polarização central discutido na seção anterior, somente as cargas da molécula de referência são sistematicamente atualizadas durante o procedimento iterativo. Com esse modelo obtivemos dipolos de 1,97 e 1,92 D calculados respectivamente para os líquidos simulados com os campos de força GXG e IK. Esses valores representam um considerável efeito de polarização de até $26 \%$ relativo ao gás. Esses resultados estão em boa concordância com as recentes estimativas de 2,05 $\pm 0,09 \mathrm{D}$ e 2,02 \pm 0,10 D para amônia líquida [208].

Tabela 8.1: Momento de dipolo calculado (MP2/aug-cc-pVTZ) para amônia líquida:

\begin{tabular}{lc}
\hline \hline Modelo & Momento de Dipolo (D) \\
\hline \hline Polarização total & \\
GXG rígido & 2,33 \\
GXG relaxado & 2,54 \\
IK rígido & 2,24 \\
IK relaxado & 2,30 \\
& \\
Polarização central & \\
GXG rígido & 1,97 \\
IK rígido & 1,92 \\
Estimativas anteriores [208] & $2,05 \pm 0,09,2,02 \pm 0,10$ \\
\hline \hline
\end{tabular}




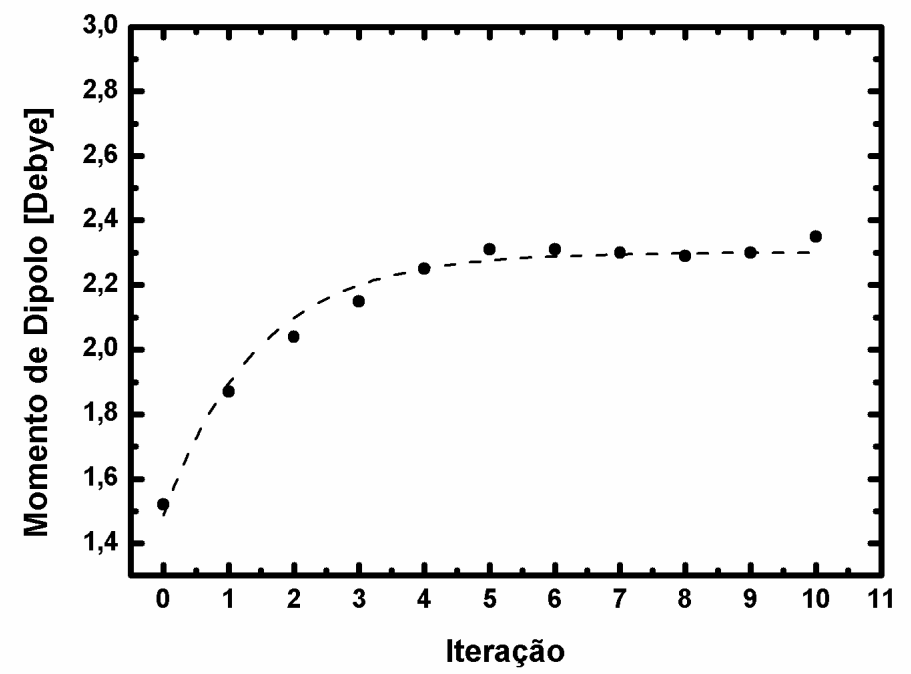

Figura 8.1: Momento de dipolo calculado (MP2/aug-cc-pVTZ) versus o número de iterações. A curva corresponde ao modelo Impey-Klein relaxado, na qual, todas as moléculas do líquido (soluto-solvente) tiveram suas cargas atualizadas (polarização total).

Utilizamos uma metodologia para inferir a polarização recíproca do solvente devido ao soluto. Nesse modelo, tanto as cargas da molécula de referência, quanto as cargas do solvente, são atualizadas durante o procedimento iterativo. Para os líquidos descritos pelos potenciais GXG e IK, obtivemos dipolos de 2, 33 e 2, 24 D, respectivamente. Note-se que a polarização recíproca soluto-solvente, prevê um aumento de até $18 \%$ em relação à polarização central.

As densidades calculadas para os líquidos simulados com os campos de força GXG e IK para a polarização central são, respectivamente, 0,634 e $0,653 \mathrm{~g} / \mathrm{cm}^{3}$. Esses valores estão em boa concordância com a densidade experimental de $0,731 \mathrm{~g} / \mathrm{cm}^{3}$. Uma das consequências da polarização recíproca soluto-solvente se reflete na considerável variação na densidade do líquido. Quando as cargas de todo o líquido são sistematicamente atualizadas durante o processo iterativo, a densidade aumenta para $0,884 \mathrm{~g} / \mathrm{cm}^{3}$. Isso ocorre porque a interação coulombiana no potencial de pares torna-se mais intensa, fazendo com que o líquido fique mais compacto.

Utilizamos o método FEG [130, 131, 132] para inferir os efeitos de relaxação geométrica sobre o momento de dipolo em amônia líquida. Uma rápida comparação entre os modelos de polarização central mostra que obtivemos dipolos de 2,54 D (potencial GXG), e 2,30 D (potencial IK). Percebe-se que o efeito de relaxação contribui com no máximo $9 \%$ em relação ao modelo rígido. A figura 8.1 mostra para o modelo IK a convergência do momento de dipolo combinando a polarização total do líquido e efeitos de relaxação de geometria. 


\section{Blindagem magnética}

Vários modelos teóricos foram utilizados para descrever quantitativamente os efeitos do solvente sobre $\sigma\left({ }^{15} \mathrm{~N}\right)$. As contribuições eletrostáticas foram consideradas através do modelo contínuo PCM [25] e da configuração média ASEC, obtida através de um procedimento iterativo [47, 48]. Esse último modelo descreve as moléculas do solvente apenas pelas cargas pontuais (CP) de seus átomos. Dessa forma, foram incluídas as 260 moléculas de $\mathrm{NH}_{3}$ que se encontravam dentro da esfera delimitada pelo raio de $12,25 \AA$. Como discutimos em uracila e piridina, a melhor maneira de descrever a blindagem magnética consiste em combinar o solvente explícito com as interações eletrostáticas provenientes das moléculas de solvente mais afastadas. Fizemos isso, primeiro considerando apenas estruturas soluto-solvente formando ligações de hidrogênio. Em outra aproximação, explicitamos as 12 moléculas de $\mathrm{NH}_{3}$ que compunham a primeira camada de solvatação ao redor do soluto. Em ambos os modelos, as moléculas explícitas foram embebidas no banho eletrostático gerado pelas amônias remanescentes tratadas como cargas pontuais.

A Tabela 8.2 mostra os resultados calculados (B3LYP/aug-pcS-3) para a blindagem magnética $\sigma\left({ }^{15} \mathrm{~N}\right)$ e para o shift $\Delta \sigma\left({ }^{15} \mathrm{~N}\right)$

$$
\Delta \sigma=\sigma_{\text {líquido }}-\sigma_{\text {gás }}
$$

Assim como em piridina, não observamos efeitos significativos provenientes da relaxação de geometria sobre a blindagem magnética, além do que, os resultados encontrados com os dois campos de força se mostraram equivalentes. Desse modo, discutiremos somente as predições obtidas com o modelo rígido GXG.

A blindagem magnética de 264,3 ppm [209] foi determinada experimentalmente em amônia isolada, à temperatura de 195, 5 K. O shift gás-líquido é drástico e mostra uma queda de -22, 6 ppm [209] na blindagem magnética. Considerar o solvente como um meio dielétrico contínuo (PCM) ou discreto (cargas pontuais) explicita apenas contribuições eletrostáticas isoladamente. Através do modelo contínuo PCM, obtivemos 262,9 ppm, o que mostra um desvio $\Delta \sigma\left({ }^{15} \mathrm{~N}\right)$ de apenas $+3,0$ ppm. É importante observar que o método produz um efeito de solvente contrário ao experimental. As mesmas observações são alcançadas para as polarizações parcial e total do líquido (veja a Tabela 8.2).

Discutimos em uracila e piridina que a melhor forma de descrever a blindagem magnética consiste em combinar modelos explícitos e eletrostáticos de solvente. Obtivemos esse efeito em dois momentos. Primeiro selecionamos estruturas soluto-solvente formando ligações de hidrogênio. Em seguida, imergimos essas estruturas no campo eletrostático das moléculas de solvente remanescentes representadas apenas por suas cargas pontuais. Esse 


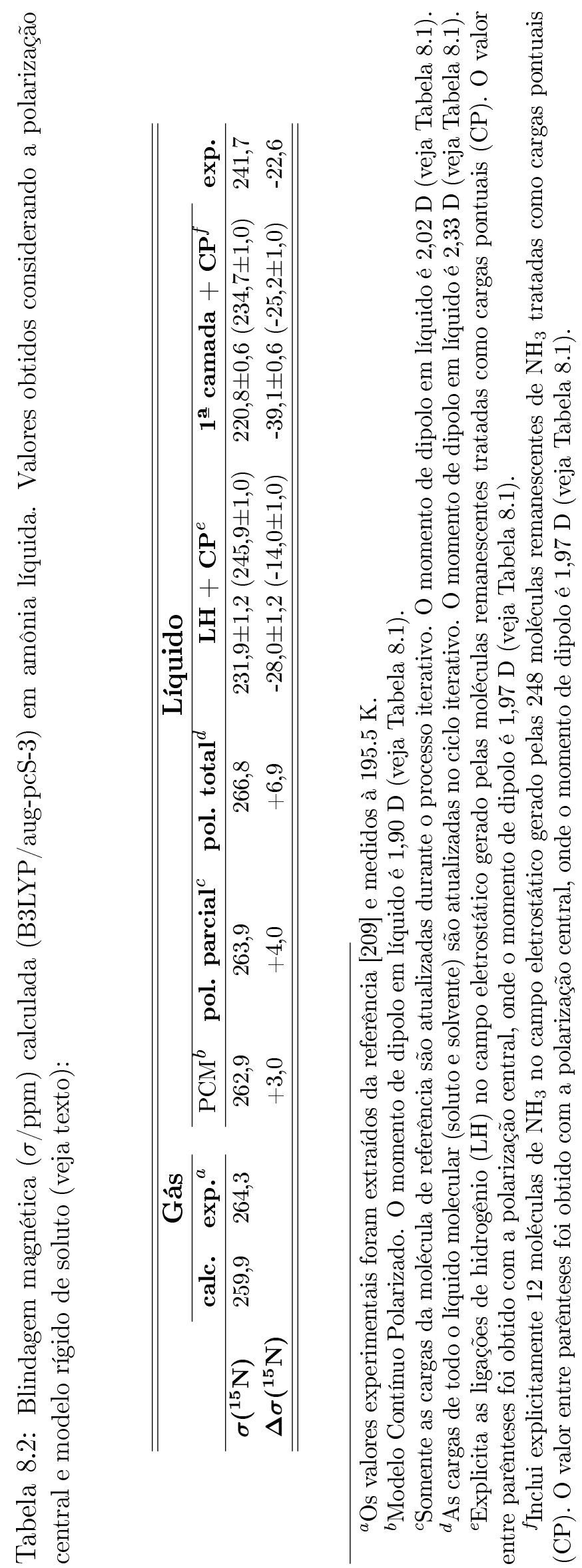


modelo é referenciado aqui como $\mathrm{LH}+\mathrm{CP}$. Em um segundo momento, incluímos no banho eletrostático as 12 moléculas de amônia encontradas na primeira camada de solvatação. Referenciamos esse modelo como $1^{\mathrm{a}}$ camada $+\mathrm{CP}$.

Para a polarização total do líquido, obtivemos com o modelo $\mathrm{LH}+\mathrm{CP}$ uma blindagem de 231,9 ppm, correspondendo ao shift de $-28,0$ ppm. Com o modelo $1^{\text {a }}$ camada + PC obtivemos um valor $220,8 \mathrm{ppm}$ para $\sigma\left({ }^{15} \mathrm{~N}\right)$ e $-39,1 \mathrm{ppm}$ para $\Delta \sigma\left({ }^{15} \mathrm{~N}\right)$. Observe que esse modelo de polarização não descreve bem o sistema, obtendo valores muito díspares do experimento.

Os melhores resultados foram obtidos para o modelo de polarização central, no qual somente as cargas do soluto são atualizadas. Esses resultados estão expostos entre parênteses na tabela 8.2. Em excelente concordância com o valor experimental, a melhor aproximação foi descrita pelo modelo $1^{\text {a }}$ camada $+\mathrm{CP}$. Neste caso, $\sigma\left({ }^{15} \mathrm{~N}\right)$ é dado por 234, 7 ppm, o que representa um efeito de solvente de $-25,2 \mathrm{ppm}$.

Embora seja um modelo mais completo, considerar a polarização recíproca do solvente devido ao soluto, não fornece bons resultados com relação ao experimento. Isso é um indício de que é preciso aprimorar agora a parte LJ do potencial intermolecular. Outro ponto importante corresponde à questões mais fundamentais. Witanowski e colaboradores $[9,10,11,12,14]$ têm discutido a dependência da blindagem magnética nuclear com relação à polaridade do solvente e a redistribuição intramolecular de cargas eletrônicas. Segundo Witanowski, a migração de cargas eletrônicas no soluto, devido ao solvente, aumenta a densidade eletrônica em determinados sítios na molécula. O acréscimo de cargas na região contribui para aumentar a blindagem magnética nuclear sobre esses sítios. A polaridade do solvente potencializa esse efeito. Ao investigar $\sigma\left({ }^{17} \mathrm{O}\right)$ em uracila e 5-fluorouracila, bem como para $\sigma\left({ }^{15} \mathrm{~N}\right)$ em piridina, observamos que há uma aparente relação entre o acúmulo de cargas e aumento de blindagem magnética em um dado sítio (veja as figuras 5.4 e 6.6). Note-se que em amônia líquida isso não ocorre. Na verdade $\Delta \sigma<0$, quando deveria ser positivo, pelo modelo defendido por Witanowski. A conclusão até o momento é que variações em $\sigma$ não podem estar relacionadas à redistribuição de cargas no soluto, mas retomaremos essa discussão no próximo capítulo.

Em piridina e pirimidina hidratadas, constatamos que o desvio gás-água $(\Delta \sigma)$ é positivo, o que implica em um aumento na blindagem magnética $\sigma\left({ }^{15} \mathrm{~N}\right)$. Em amônia, observase que $\Delta \sigma<0$. Piridina e pirimidina são sistemas que fazem ligações de hidrogênio apenas aceitando o hidrogênio do solvente, amônia, por outro lado é capaz de fazê-lo tanto aceitando quando doando hidrogênios, como pode ser visto nas figuras 8.2 e 8.3. Para o oxigênio do fenol, tem sido observado que seu caráter aceitador $(\mathrm{O} \cdots \mathrm{HX})$ e doador $(\mathrm{OH} \cdots \mathrm{X})$ em ligações de hidrogênio produz desvios opostos em seu espectro de absorção eletrônica [46, 210]. Assim, é fundamental discutir se o mesmo comportamento competi- 
tivo é o responsável pelo desvio $\Delta \sigma<0$.
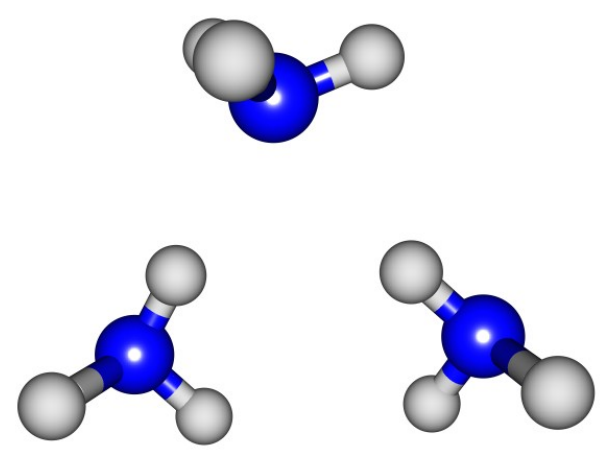

Figure 8.2: Amônia de referência (topo) formando ligações de hidrogênio com outras duas amônias. Essa estrutura corresponde a $51 \%$ das estruturas onde o soluto apenas aceita o hidrogênio do solvente.

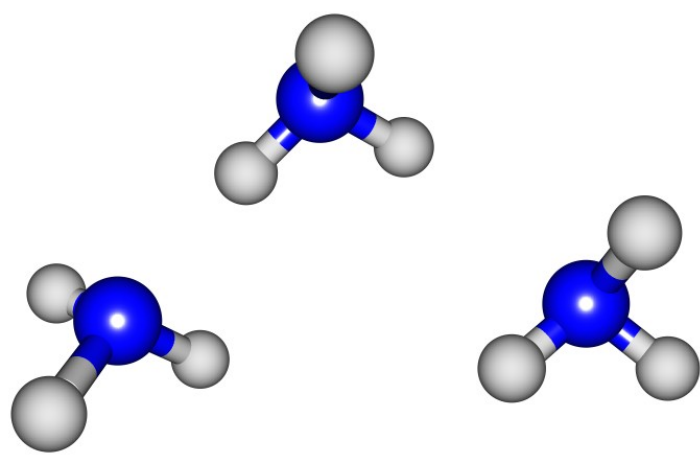

Figure 8.3: Amônia de referência (topo) interagindo explicitamente com outras duas moléculas. Essa estrutura corresponde a 47,4\% das estruturas onde o soluto apenas doa o hidrogênio ao solvente.

Obtivemos das simulações MC estruturas soluto-solvente fortemente ligadas através de ligações de hidrogênio e, embebidas no banho eletrostático das demais moléculas do solvente, estas consideradas apenas como cargas pontuais. Essas estruturas foram obtidas em dois estágios, considerando situações em que o soluto participa ( $a$ ) apenas como doador $(\mathrm{NH} \cdots \mathrm{N})$ e em separado, $(b)$ como aceitador $(\mathrm{N} \cdots \mathrm{H})$ em ligações de hidrogênio. O objetivo é apenas quantificar essas contribuições. Os cálculos de mecânica quantica executados sobre esse modelo são descritos em nível de teoria B3LYP/aug-pcS-2 ${ }^{\text {(soluto) }} / \mathrm{pcS}-2^{\text {(solvente) }}$. Nesta notação, os índices superiores indicam as bases usadas no soluto e no solvente, respectivamente. Os resultados estão dispostos na tabela 8.3. Obtivemos médias de $-12,1 \pm 0,9$ e 9,2 \pm 0, 8 ppm para os desvios gás-líquido ao considerar estruturas doadoras e aceitadoras, respectivamente. Observe-se que as contribuições para a blindagem magnética quando o nitrogênio comporta-se como doador em uma LH superam suas contribuições como aceitador em até $30 \%$. Os resultados são conclusivos e mostram que a 
Table 8.3: Contribuições para a blindagem magnética $(\sigma / \mathrm{ppm})$ sobre o nitrogênio considerando a participação do soluto em ligações de hidogênio:

\begin{tabular}{lcc}
\hline \hline & $\boldsymbol{\sigma}_{\mathbf{L H}+\mathbf{C P}}$ & $\boldsymbol{\Delta} \boldsymbol{\sigma}_{\mathbf{L H}+\mathbf{C P}}$ \\
\hline \hline Aceitador $(\mathbf{N} \cdots \mathbf{H})^{a}$ & $250,7 \pm 0,8$ & $-9,2 \pm 0,8$ \\
Doador $(\mathbf{N H} \cdots \mathbf{N})^{b}$ & $247,5 \pm 0,9$ & $-12,1 \pm 0,9$ \\
\hline \hline
\end{tabular}

(a) Contribuições obtidas considerando a participação do soluto apenas como aceitador $(\mathrm{N} \cdots \mathrm{H})$ em ligações de hidrogênio. (b) Contribuições obtidas considerando a participação do soluto apenas como doador $(\mathrm{NH} \cdots \mathrm{N})$ em ligações de hidrogênio.

maior contribuição para o decrécimo da blindagem $\sigma\left({ }^{15} \mathrm{~N}\right)$ em solução é o caráter doador do nitrogênio do soluto.

Assegurar a convergência estatística na descrição de sistemas líquidos é um tópico importante. A figura 8.4 mostra a convergência obtida para o shift gás-líquido $\Delta \sigma\left({ }^{15} \mathrm{~N}\right)$ como função do número de configurações MC. A rápida convergência para um valor médio é uma consequência direta da escolha de configurações estatisticamente descorrelacionadas. A figura 8.5 mostra o histograma da distribuição estatística do shift $\Delta \sigma\left({ }^{15} \mathrm{~N}\right)$.

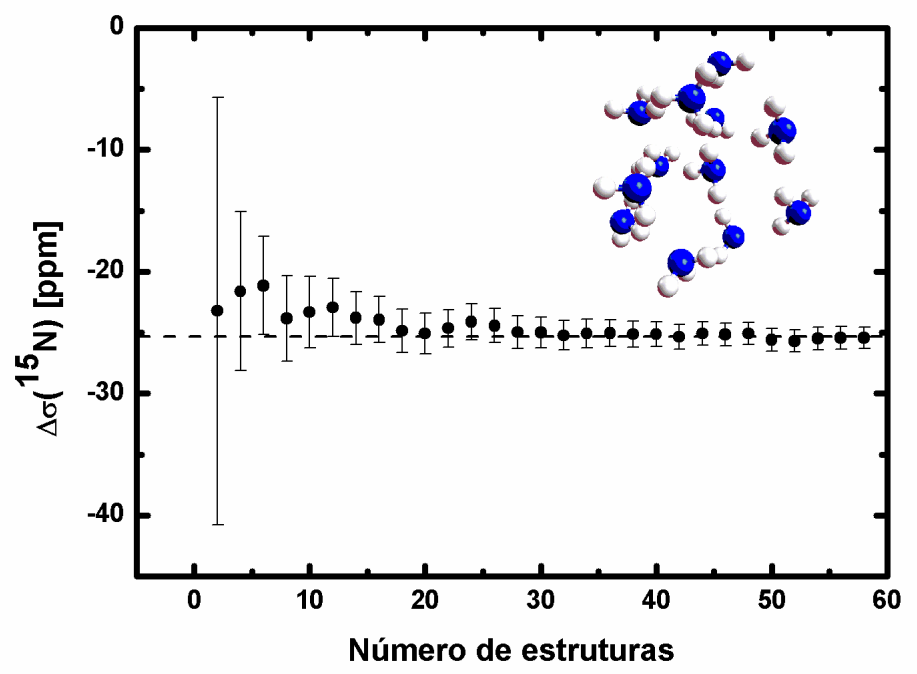

Figura 8.4: Desvio médio gás-líquido $\Delta \sigma\left({ }^{15} \mathrm{~N}\right)$ obtido para a primeira camada de solvatação. A linha tracejada corresponde ao valor médio $-25,2 \pm 1,0 \mathrm{ppm}$. A incerteza representa o erro estatístico. 


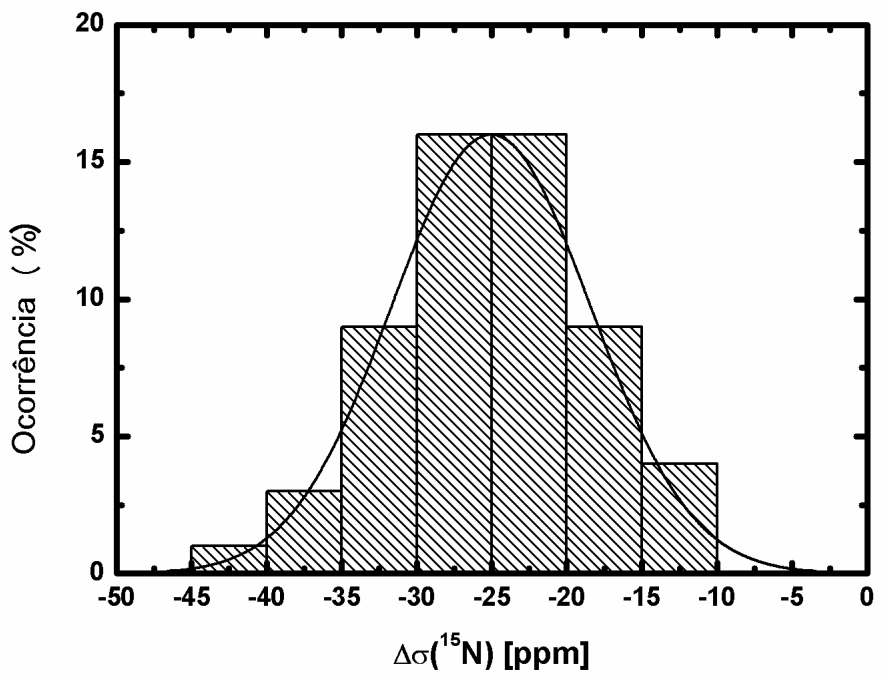

Figura 8.5: Histograma obtido para o desvio médio gás-líquido $\Delta \sigma\left({ }^{15} \mathrm{~N}\right)$.

\section{Acoplamento intramolecular entre spins nucleares}

A tabela 8.4 mostra os valores calculados e observados para os acoplamentos ${ }^{1} \mathrm{~J}(\mathrm{~N}-\mathrm{H})$ e ${ }^{2} J(\mathrm{H}-\mathrm{H})$. Ambos mostram que a contribuição mais importante provém do termo de contato de Fermi (FC). Para o acoplamento entre hidrogênios é interessante notar que os termos diamagnético (DSO) e paramagnético (PSO) têm magnitudes consideráveis quando comparados ao acoplamento ${ }^{1} J(\mathrm{~N}-\mathrm{H})$.

Correções vibracionais sobre os valores experimentais também estão incluídas na tabela 8.4. De modo geral as estimativas teóricas superestimam ligeiramente as constantes de acoplamento em relação aos experimentos mais recentes reportados por Wasylishen e Friedrish [199]. Como exemplo, considere o resultado $-64,35 \mathrm{~Hz}$ para o acoplamento ${ }^{1} \mathrm{~J}(\mathrm{~N}-\mathrm{H})$ obtido com o modelo GXG relaxado. Esse valor diverge $2,45 \mathrm{~Hz}$ do valor experimental corrigido vibracionalmente para $-61,9 \mathrm{~Hz}$. O modelo IK relaxado apresenta a mesma tendência, diferindo das observações por apenas 2,02 Hz. Para o acoplamento ${ }^{2} J(\mathrm{H}-\mathrm{H})$, essas diferenças, respectivamente calculadas com os modelos relaxados GXG e IK são 2,71 e 2,26 Hz. Adicionalmente é possível notar na tabela 8.4 que o efeito de relaxação afeta diferentemente os acoplamentos nucleares. Quando os modelos relaxados são comparados aos modelos rígidos, percebe-se em módulo um decréscimo no acoplamento ${ }^{1} J(\mathrm{~N}-\mathrm{H})$ e um aumento em ${ }^{2} J(\mathrm{H}-\mathrm{H})$. As figuras 8.6 e 8.7 mostram a evolução dos acoplamentos com relação à polarização eletrônica do soluto isoladamente, bem como combinada aos efeitos de relaxação geométrica. Embora haja pequenas diferenças da ordem de $2,0 \mathrm{~Hz}$, os modelos rígidos e relaxados mostram comportamentos similares para a constante ${ }^{2} J(\mathrm{H}-\mathrm{H})$. Por outro lado, a figura 8.7 mostra que os efeitos de relaxação e 


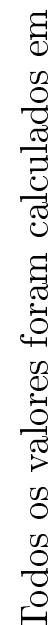

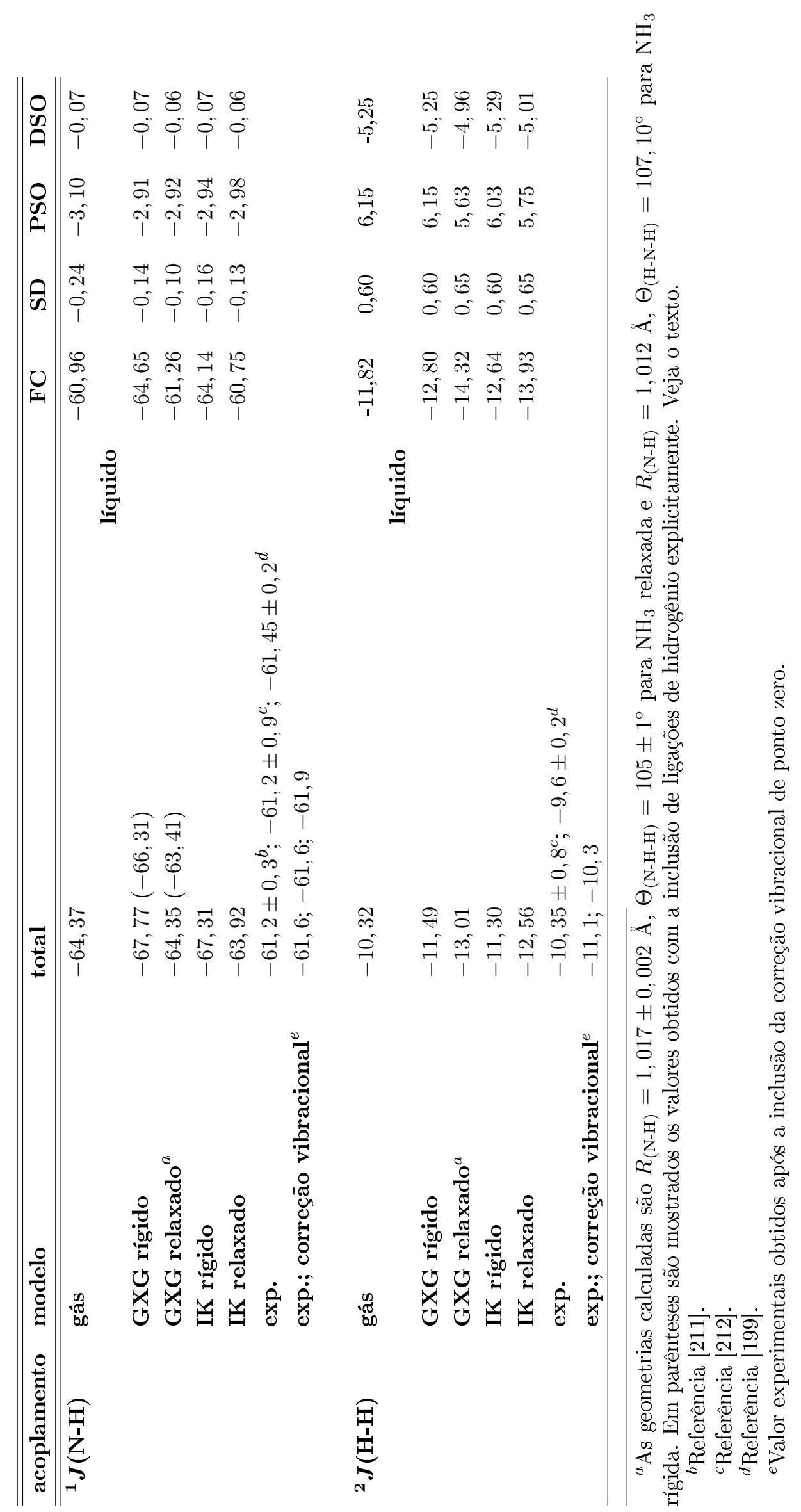


polarização são contrários com relação ao acoplamento ${ }^{1} J(\mathrm{~N}-\mathrm{H})$.

O procedimento iterativo usado aqui apenas inclui contribuições eletrostáticas. Por outro lado, amônia constitui um líquido bem estruturado e capaz de formar um número considerável de ligações de hidrogênio. Isso faz com que investigar o efeito da inclusão de moléculas explícitas do solvente sobre as constantes de acoplamento seja um problema importante. A formação de ligações de hidrogênio foi sistematicamente discutida e incluída considerando critérios energéticos e geométricos. Através desses critérios foi possível incluir estruturas em que o soluto $\mathrm{NH}_{3}$ atua simultaneamente como doador e aceitador de prótons. Em todos as configurações, o sistema supermolecular foi imerso no banho eletrostático gerado pelas moléculas de amônia remanescentes, tratadas como cargas pontuais. Os resultados para o acoplamento ${ }^{1} J(\mathrm{~N}-\mathrm{H})$ são mostrados (entre parênteses) na tabela 8.4, para o modelo GXG em suas variantes rígida e relaxada. Conclui-se que a inclusão de ligações de hidrogênio afeta muito pouco o acoplamento intramolecular. Embora sejam contribuições muito pequenas, variando entre 1,0 e 1,5 Hz, a inclusão de ligações de hidrogênio aponta na direção do valor experimental.

Os resultados experimentais listados na tabela 8.4 mostram que o acoplamento ${ }^{1} J(\mathrm{~N}-\mathrm{H})$ é essencialmente o mesmo nas fases líquida e gasosa. Considerando as aproximações utilizadas, apenas os modelos relaxados são capazes de reproduzir o experimento. O modelo relaxado IK, por exemplo, apresenta um shift gás-líquido de apenas $0,45 \mathrm{~Hz}$, enquanto o desvio de $-2,94 \mathrm{~Hz}$ é obtido com o modelo rígido. Adicionalmente, os modelos usados permitem estimar separadamente as contribuições do efeito da relaxação de geometria e polarização. A tabela 8.5 encerra os dados obtidos. Para o acoplamento ${ }^{1} J(\mathrm{~N}-\mathrm{H})$, o modelo GXG avalia o efeito puro da polarização (EPP) observando o shift gás-líquido $\Delta^{1} J(\mathrm{~N}-\mathrm{H})$ de $-3,4 \mathrm{~Hz}$. Com o mesmo modelo, o efeito puro da geometria (EPG) é obtido tomando a diferença entre os modelos relaxado e rígido, dada por $3,4 \mathrm{~Hz}$. Como consequência, o shift total $\Delta^{1} J(\mathrm{~N}-\mathrm{H})$ é nulo. Isso mostra que os efeitos de polarização e relaxação de geometria tendem a se cancelar.

Com relação ao acoplamento ${ }^{2} J(\mathrm{H}-\mathrm{H})$, um shift total de $-2,7 \mathrm{~Hz}$ é obtido. Contreras e Peralta [154] estudaram os efeitos da variação de geometria sobre as constantes de acoplamento em amônia. Como efeito geral, foi observado que ${ }^{1} J(\mathrm{~N}-\mathrm{H})$ diminui e ${ }^{2} J(\mathrm{H}-\mathrm{H})$ aumenta quando o ângulo piramidal aumenta. Nos modelos rígido e relaxado usados, esses ângulos são dados por 107, 1 e $105^{\circ}$, respectivamente. As predições de Contreras e Peralta estão em concordância com os resultados mostrados nas tabelas 8.4 e 8.5. 


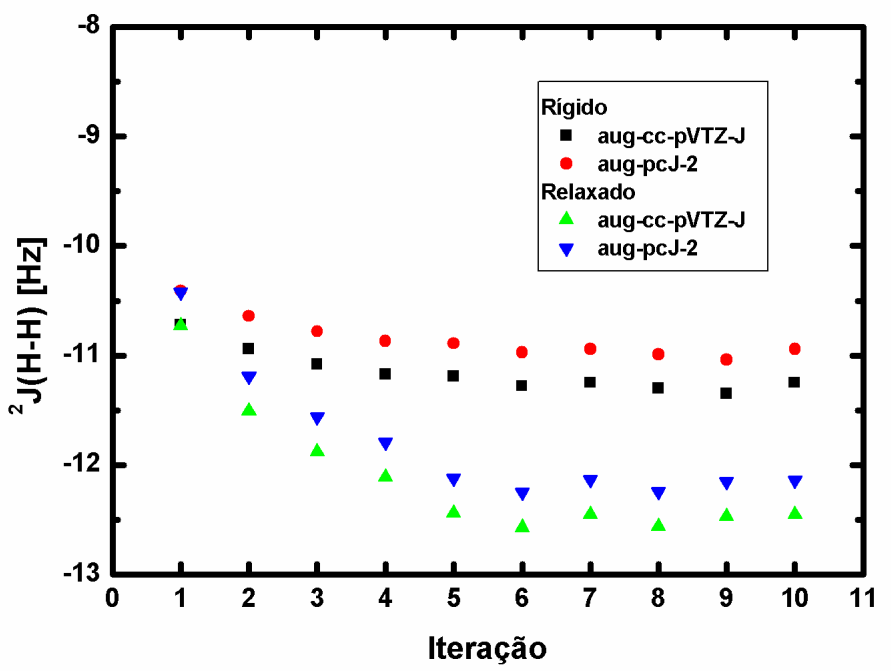

Figura 8.6: Evolução do acoplamentos indireto ${ }^{2} J(\mathrm{H}-\mathrm{H})$ com relação à polarização do soluto para o modelo Impey-Klein rígido e relaxado. Foram consideradas geometrias rígidas e relaxadas e bases aug-cc-pVTZ-J e aug-pcJ-2.

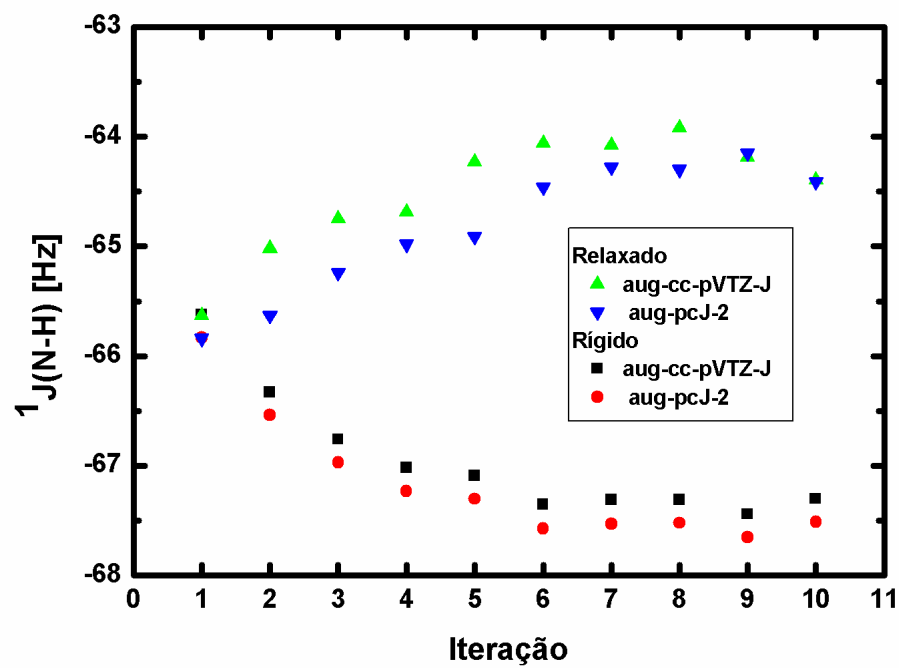

Figura 8.7: Evolução dos acoplamento indireto ${ }^{1} J(\mathrm{~N}-\mathrm{H})$ com relação à polarização do soluto para o modelo Impey-Klein rígido e relaxado. Foram consideradas geometrias rígidas e relaxadas e bases aug-cc-pVTZ-J e aug-pcJ-2. 
Tabela 8.5: Efeito puro da polarização (EPP) e relaxação (EPG) para o efeito total do solvente sobre as constantes de acoplamento:

\begin{tabular}{llcc}
\hline \hline contribuição & acoplamento & modelo IK & modelo GXG \\
\hline \hline EPP & $\Delta^{1} J(\mathrm{~N}-\mathrm{H})_{\text {gás-líquido }}$ & $-2,9$ & $-3,4$ \\
& $\Delta^{2} J(\mathrm{H}-\mathrm{H})_{\text {gás-líquido }}$ & $-1,0$ & $-1,2$ \\
\multirow{2}{*}{ EPG } & $\Delta^{1} J(\mathrm{~N}-\mathrm{H})_{\text {rígido-relaxado }}$ & 3,4 & 3,4 \\
& $\Delta^{2} J(\mathrm{H}-\mathrm{H})_{\text {rígido-relaxado }}$ & $-1,3$ & $-1,5$ \\
\multirow{4}{*}{ total } & $\Delta^{1} J(\mathrm{~N}-\mathrm{H})$ & 0,5 & 0,0 \\
& $\Delta^{2} J(\mathrm{H}-\mathrm{H})$ & $-2,3$ & $-2,7$ \\
\hline \hline
\end{tabular}

\subsection{Conclusões}

Em amônia líquida, foram investigados os efeitos do meio sobre os acoplamentos indiretos entre spins nucleares ${ }^{1} J(\mathrm{~N}-\mathrm{H})$ e ${ }^{2} J(\mathrm{H}-\mathrm{H})$, bem como sobre a blindagem magnética nuclear $\sigma\left({ }^{15} \mathrm{~N}\right)$. Efeitos de polarização e relaxação de geometria foram inferidos de modo a avaliar suas contribuições individuais sobre as constantes magnéticas. Modelos discretos, contínuos e explícitos de solventes foram utilizados.

Notamos que a polarização eletrônica do soluto devido ao meio é fundamental para a descrição das constantes magnéticas nucleares em geral. Por outro lado, embora efeitos de relaxação geométrica sejam fundamentais na descrição dos acoplamentos indiretos entre spins nucleares, os mesmos têm pouca influência para a blindagem magnética.

Para o acoplamento ${ }^{1} J(\mathrm{~N}-\mathrm{H})$, os resultados variam entre $-67,8$ e $-63,9 \mathrm{~Hz}$, dependendo do modelo utilizado. Esses dados estão em boa concordância com a observação experimental de $-61,25 \mathrm{~Hz}$. O shift gás-liquido $\Delta^{1} J(\mathrm{~N}-\mathrm{H})$ calculado também reproduz o experimento. Para o acoplamento entre prótons ${ }^{2} J(\mathrm{H}-\mathrm{H})$ os resultados variam entre $-10,6$ e -13,01 Hz. O shift vapor-líquido $\Delta^{2} J(\mathrm{H}-\mathrm{H})$ não pôde ser avaliado experimentalmente, mas estimamos um valor de $-2,7 \mathrm{~Hz}$. As constantes de acoplamento foram igualmente bem descritas por modelos discretos de solvente, bem como pela inclusão de moléculas explícitas à interação eletrostática. Isso acontece por que a maior contribuição para o acoplamento vem do termo de contato de Fermi, que depende fortemente dos elétrons em orbitais atômicos de simetria $s$. Esses elétrons são levemente perturbados pelas interações de van der Waals. Essa característica permite tratar líquidos moleculares maiores sem a necessidade de estender as funções de onda sobre o solvente. Como o acoplamento entre spins nucleares demanda funções bases excessivamente grandes, essa propriedade 
representa uma importante economia computacional.

Os modelos discutidos acima também foram aplicados na descrição da blindagem magnética nuclear $\sigma\left({ }^{15} \mathrm{~N}\right)$. Em particular, modelos eletrostáticos de solvente não apresentam uma boa descrição do sistema. Os valores obtidos com essas aproximações para o shift $\Delta \sigma\left({ }^{15} \mathrm{~N}\right)$ predizem desvios com sinais opostos ao experimento. Uma descrição mais consistente pode ser obtida acoplando explicitamente interações eletrostáticas e ligações de hidrogênio soluto-solvente. Obtivemos com essa aproximações desvios $\Delta \sigma\left({ }^{15} \mathrm{~N}\right)$ de $-25,2$ ppm, em boa concordância com o valor experimental de $-22,6$ ppm. Observamos mais uma vez que efeitos de relaxação geométrica sobre a blindagem magnética são muito pequenos e que a polarização eletrônica é crucial na descrição de propriedades magnética nucleares.

Temos visto, até o momento, e estudos recentes mostram que o aumento na blindagem magnética nuclear, em um dado sítio molecular, está diretamente correlacionado ao acúmulo de cargas sobre o sítio. Todos os sistemas estudados têm em comum o caráter aceitador, quando envolvidos em ligações de hidrogênio intermoleculares. Em amônia, embora haja realmente um acúmulo de cargas sobre o nitrogênio, a blindagem magnética $\sigma\left({ }^{15} \mathrm{~N}\right)$ diminui, quando em solução, contrariando a teoria vigente. Fundamentalmente, conclui-se que o sinal da blindagem magnética sobre determinados núcleos não é determinado exclusivamente pela migração das cargas eletrônicas do soluto, normalmente potencializada pela polaridade do meio, mas há indícios de que o caráter prótico do soluto pode ser preponderante. 


\section{Capítulo 9}

\section{Formamida líquida}

\subsection{Introdução}

Amidas têm sido objeto de vários trabalhos em ressonância magnética nuclear devido a sua importância como protótipo em ligações peptídicas, bem como por sua habilidade na formação de ligações de hidrogênio intermoleculares. Em particular, Kamlet e Taft desenvolveram um método de comparação solvatocrômica a fim de desvencilhar e racionalizar os efeitos do solvente sobre a blindagem magnética nuclear $\sigma\left({ }^{15} \mathrm{~N}\right)$ em amidas. No entanto, o espectro RMN do oxigênio tem recibo particular atenção devido a sua extrema sensibilidade à solvatação e efeitos substitucionais [16, 17, 98]. Como exemplo, Burgar, Amour e Fiat [213, 214] foram os primeiros a idealizar um modelo simples para separar as diferentes contribuições sobre $\sigma\left({ }^{17} \mathrm{O}\right)$ devido à formação de ligações de hidrogênio em vários sítios em amidas.

Com base nos parâmetros Kamlet-Abboud-Taft, Gerothanassis e Vakka [17, 56, 49, 55] propuseram um modelo mais eficiente de solvatação para descrever a blindagem magnética $\sigma\left({ }^{17} \mathrm{O}\right)$ em amidas e peptídeos. Sistematicamente tem-se observado que interações de longo alcance e a solvatação do oxigênio em amidas são responsáveis por modificações drásticas na blindagem $\sigma\left({ }^{17} \mathrm{O}\right)$. Gerothanassis e Vakka concluem ainda que a solvatação do hidrogênio não induz grandes contribuições sobre $\sigma\left({ }^{17} \mathrm{O}\right)$ em amidas.

Com relação ao espectro RMN do ${ }^{15} \mathrm{~N}$, muitos avanços se devem às contribuições contínuas de Witanowski e Webb [9, 10, 11, 12, 13, 14, 15]. Contudo, principalmente com relação à estrutura molecular, o espectro $\mathrm{RMN}$ do ${ }^{15} \mathrm{~N}$ ainda hoje é foco de vários trabalhos experimentais [215, 216, 217, 218]. Neste trabalho, além de investigar os efeitos do solvente sobre a blindagem magnética nuclear $\sigma\left({ }^{15} \mathrm{~N}\right)$, revisitamos os modelos de solvatação propostos por Burgar, Amour, Fiat, Gerothanassis e Vakka a fim de desenvolver uma metodologia independente de parâmetros empíricos. Após separar e racionalizar as contribuições do meio sobre a blindagem magnética nuclear $\sigma\left({ }^{17} \mathrm{O}\right)$ em amidas mostraremos, 
sistematicamente que a solvatação do hidrogênio tem papel crucial, ao contrário da teoria vigente.

\subsection{Detalhes computacionais}

Utilizamos novamente o procedimento $\mathrm{s}-\mathrm{QM} / \mathrm{MC}$ para simular o líquido molecular no ensemble NPT, com 501 moléculas de formamida sob condições normais de temperatura e pressão. As interações moleculares foram mediadas pelos potenciais de Lennard-Jones (LJ) e Coulomb com três parâmetros para cada sítio atômico. Os parâmetros LJ foram extraídos do banco de dados OPLS [79] desenvolvido por Jorgensen e colaboradores. Já as cargas atômicas foram obtidas em nível de cálculo MP2/aug-cc-pVQZ, usando o ajuste eletrostático CHELPG [103], que minimiza o potencial clássico.

Os efeitos da polarização eletrônica do soluto novamente foram avaliados considerando modelos contínuos e um procedimento iterativo. Os modelos contínuos têm origens nas ideias de Kirkwood [24] e Onsager [23] e tomam o solvente como um meio contínuo descrito por sua constante dielétrica. O soluto é encerrado em uma cavidade imersa no meio dielétrico. O modelo contínuo polarizado (PCM) [25] é uma evolução natural nos modelos contínuos e tem dado bons resultados na predição de propriedades magnéticas nucleares [37]. O modelo iterativo [47, 48] permite equilibrar as cargas eletrônicas do soluto na presença do solvente. Essa metodologia também tem sido usada com sucesso no estudo de constantes magnéticas nucleares em vários compostos de interesse industrial e biológico [36, 45, 121, 139, 194].

Uma evolução do procedimento iterativo descrito acima utiliza o método do gradiente de energia livre [130, 131, 132] em conjunto com a metodologia s-QM/MM para obter a geometria molecular do soluto mediante ao solvente. Uma discussão mais detalhada sobre a aproximação FEG pode ser encontrada nos trabalhos originais de Nagaoka e colaboradores [130, 131, 132]. Recentemente, o método FEG foi implementado no programa Diceplayer [138], o qual faz uma interface entre o programa de simulações MC Dice [101] e programas de mecânica quântica. Temos utilizado a metodologia FEG para estudar a dependência de efeitos geométricos sobre as constantes magnéticas nucleares em amônia líquida [139] e piridina hidratada [194]. Em formamida líquida utilizamos novamente essa metodologia para estudar o efeito do solvente sobre as blindagens magnéticas $\sigma\left({ }^{17} \mathrm{O}\right)$ e $\sigma\left({ }^{15} \mathrm{~N}\right)$. As constantes magnéticas nucleares de interesse foram obtidas utilizando as bases $6-311++\mathrm{G}(d, p)$ em concomitância com as teorias MP2 e DFT. Embora haja bases específicas para os estudo propriedades magnéticas, Ribeiro et al [151] têm mostrado que essas bases podem reproduzir situações não físicas. Devido aos bons resultados obtidos para blindagem magnética nuclear [151, 194], explicando desacordos entre teoria e ex- 
perimento, optamos por usar as bases $6-311++\mathrm{G}(d, p)$. A independência com relação a origem foi evitada usando o método GIAO [84]. Todas as simulações clássicas MC e cálculos de mecânica quântica foram respectivamente executados pelos programas DICE [101] e Gaussian 03 [118].

\subsection{Resultados e discussões}

\section{Momento de dipolo}

Discutiremos agora a polarização eletrônica do soluto induzida pelo solvente. Os principais resultados estão expostos na tabela 9.1. Vários trabalhos experimentais apontam um momento de dipolo em torno de 3,7 D [148, 219, 220, 221, 222] para o gás. Para a molécula isolada obtivemos um dipolo de 3,9 D, em excelente concordância com o experimento. Embora não haja na literatura dados experimentais sobre o dipolo do líquido, utilizamos modelos contínuos e discretos de solvente para estimar essa propriedade. Utilizando a geometria (MP2/aug-cc-pVQZ) obtida pelo modelo contínuo PCM, em mesmo nível de aproximação, calculamos o momento de dipolo de $5,1 \mathrm{D}$, o qual implica em uma polarização de $30 \%$ em relação à molécula isolada.

Tabela 9.1: Momento de dipolo ( $\mu / \mathrm{D})$ e cargas eletrônicas ( $q / e)$ calculadas (MP2/aug-cc-pVQZ) em formamida. Também são mostradas as densidades $\left(\rho / \mathrm{g} \cdot \mathrm{cm}^{-3}\right)$ obtidas com diferentes modelos de polarização eletrônica:

\begin{tabular}{cccccc}
\hline \hline & Gás & PCM $^{a}$ & ASEC-Rig $^{b}$ & ASEC-Rel $^{c}$ & Exp. (Gás) $^{\text {ESE }}$ \\
\hline $\boldsymbol{\mu}$ & 3,9 & 5,1 & 4,8 & 5,0 & 3,7 D [148] \\
& & & & & \\
$\mathbf{C}$ & 0,666868 & 0,687968 & 0,722154 & 0,725700 & \\
$\mathbf{N}$ & $-0,885787$ & $-0,863772$ & $-0,920060$ & $-0,904781$ & \\
$\mathbf{O}$ & $-0,544987$ & $-0,658122$ & $-0,644495$ & $-0,656657$ & \\
& & & 1,1082 & 1,1091 & $1,1334[148]$ \\
$\boldsymbol{\rho}$ & & & & & \\
\hline \hline
\end{tabular}

(a) Geometria PCM/MP2/aug-cc-pVQZ. (b) Geometria (MP2/aug-cc-pVQZ) obtida para a molécula isolada. (c) Além das cargas eletrônicas do soluto, esse modelo usa o método do gradiente de energia livre (FEG) para relaxar também a geometria do soluto na presença do solvente (veja texto).

Utilizamos o procedimento iterativo para estimar a contribuição do solvente sobre a polarização eletrônica do soluto. O modelo ASEC-Rig, refere-se ao procedimento iterativo administrado sem considerar efeitos de relaxação geométrica. A geometria usada nesse 
procedimento foi obtida para o sistema isolado em nível de aproximação (MP2aug-ccpVQZ). O modelo ASEC-Rel utiliza a aproximação FEG [130, 131, 132], como descrita na seção anterior, para inferir efeitos de relaxação de geometria induzidos pelo meio. Com o modelo rígido de geometria (ASEC-Rig) obtivemos um momento dipolar de 4, 8 D, o que representa um efeito do solvente de $23 \%$ em relação à molécula isolada. Já o modelo ASEC-Rel prediz um momento de dipolo de 5, 0 D, representando uma polarização eletrônica de $28 \%$. Note-se que os modelos ASEC-Rel e PCM estão em boa concordância, certamente por considerarem geometrias obtidas no ambiente líquido.

A densidade experimental da formamida líquida é $1,1334 \mathrm{~g} / \mathrm{cm}^{3}$. Nossas simulações utilizando as polarizações iterativas ASEC-Rig e ASEC-Rel mostram densidades de 1, 1082 e $1,1091 \mathrm{~g} \mathrm{~g} / \mathrm{cm}^{3}$, respectivamente. As pequenas diferenças nas densidades, com relação ao experimento, provavelmente surgem por não termos considerado a polarização recíproca do solvente devido soluto. Contudo, está claro que as descrições ASEC-Rig e ASEC-Rel estão em boa concordância com experimento.

\section{Ligações de hidrogênio}

Como tem sido discutido em trabalhos recentes [194], o número de coordenação do solvente sobre o soluto, bem como a formação de ligações de hidrogênio, são quantidades significativamente sensíveis à polarização eletrônica do soluto devido ao meio. Informações preciosas sobre a distribuição do solvente são facilmente obtidas das funções de distribuição radial (RDF) do sistema.

Considere o modelo ASEC-Rig, contudo, não levaremos em conta efeitos de geometria. O primeiro passo do ciclo iterativo corresponde à situação em que ainda não foram incluídos os efeitos de polarização. Naturalmente, o último passo do ciclo refere-se ao máximo de polarização, no qual a redistribuição das cargas eletrônicas do soluto está convergida. Comparando esses extremos discutiremos brevemente a influência da polarização eletrônica sobre a estrutura líquida. As principais informações estão dispostas na tabela 9.2. A primeira camada de solvatação também não sofre alterações significativas. Para o caso não polarizado, os limites para a primeira camada estendem-se desde 1,5 até $4,7 \AA$, tendo sido contabilizadas 14 formamidas dentro dessa região esférica. Para o caso polarizado, a primeira camada é sensivelmente menor. Com limites entre 1,3 e 4,1A, é composta por apenas 13 formamidas.

As maiores contribuições da redistribuição eletrônica no soluto são diretamente observadas sobre a formação de ligações de hidrogênio no líquido. Ligações de hidrogênio são melhor discutidas combinando critérios geométricos e energéticos [149, 152, 153]. Aqui, caracterizamos uma ponte de hidrogênio quando $R_{\mathrm{O}-\mathrm{H}} \leq 5,0 \AA, \alpha(\mathrm{RO}-\mathrm{H}) \leq 35^{\circ}$ e $E=-4,0 \mathrm{kcal} / \mathrm{mol}$. 
Para o caso não polarizado, em $18 \%$ das estruturas MC geradas, obtivemos apenas uma ligação de hidrogênio. A situação mais frequênte corresponde à formação de até duas ligações, com $35 \%$ das ocorrências. Com até três ligações, observamos $33 \%$ dos casos. Finalmente, as ocorrências menos frequêntes correspondem à formação de quatro e cinco ligações de hidrogênio, respectivamente, com 12 e $2 \%$ das estruturas. Obtivemos no total uma média de 1,97 ligações por estrutura.

Para o caso polarizado as mudanças são brutais. Obtivemos 0,5\% das configurações com apenas uma ligação de hidrogênio, enquanto duas ligações são observadas em somente 9, 9 \% dos casos. As situações de maior ocorrência são encontradas para estruturas onde há três e quatro ligações, respectivamente, com 40 e 39,7 \% das configurações. Os casos menos frequentes correspondem à formação de cinco e seis (antes não observadas) ligações de hidrogênio, respectivamente, com 9,9 e 0,5\% das ocorrências. Obtivemos uma média 2,94 ligações de hidrogênio por estrutura.

Tabela 9.2: Contagem (\%) da formação de ligações de hidrogênio em formamida líquida:

\begin{tabular}{ccc}
\hline \hline Número de ligações & Não polarizado & Polarizado \\
\hline $\mathbf{1}$ & 18 & 0,5 \\
$\mathbf{2}$ & 35 & 9,9 \\
$\mathbf{3}$ & 33 & 40,0 \\
$\mathbf{4}$ & 12 & 39,7 \\
$\mathbf{5}$ & 2,0 & 9,9 \\
$\mathbf{6}$ & - & 0,5 \\
Média & 1,97 & 2,94 \\
\hline \hline
\end{tabular}

\section{Blindagem magnética $\sigma\left({ }^{17} \mathrm{O}\right)$}

Discutiremos agora o efeito do solvente sobre a blindagem magnética nuclear $\sigma\left({ }^{15} \mathrm{O}\right)$ em formamida líquida. Convertemos os desvios químicos experimentais $(\delta)$ para a blindagem magnética $(\sigma)$ através da escala absoluta de conversão $(\sigma=287,5 \mathrm{ppm}-\delta)$ proposta por Wasilyshen [119]. A tabela 9.3 lista os principais resultados. Para o gás, Gerothanassis e colaboradores $[17,49,55,56]$ têm sistematicamente reportado a blindagem de $-35,5$ ppm sobre o isótopo ${ }^{17} \mathrm{O}$. Em nível de aproximação MP2 e B3LYP, obtivemos blindagens de $-35,7$ e $-92,2$ ppm para a molécula isolada. Observe que a predição perturbativa concorda muito bem com o experimento.

O espectro RMN do oxigênio é conhecido por ser extremamente sensível ao ambiente $[16,17]$. São reportados $24,0 \mathrm{ppm}$ para a blindagem magnética $\sigma\left({ }^{17} \mathrm{O}\right)$ em formamida líquida [50]. Utilizamos vários modelos de solvente para aferir o efeito do solvente so- 
bre essa propriedade molecular. O modelo contínuo $\mathrm{PCM} / \mathrm{MP} 2 / 6-311++\mathrm{G}(d, p)$, por exemplo, prediz uma blindagem magnética de 12,4 ppm, em pobre concordância com o experimento.

Utilizamos o procedimento iterativo sem e com relaxação geométrica para modelar $\sigma\left({ }^{17} \mathrm{O}\right)$. Esses modelos estão referenciados na tabela 9.3, respectivamente, como ASECRig e ASEC-Rel. O modelo ASEC-Rig mantém rígida a geometria obtida em vácuo ao longo de todos os passos iterativos, portanto, leva em conta somente efeitos da polarização eletrônica do soluto devido ao meio. Por sua vez, o modelo ASEC-Rel, sistematicamente atualiza a geometria da molécula de referência entre os passos iterativos. Note-se que essa aproximação combina efeitos da polarização eletrônica e relaxação geométrica do soluto devido ao solvente. O modelo ASEC-Rig prediz uma blindagem magnética de 5,1 ppm sobre o isótopo ${ }^{17} \mathrm{O}$, enquanto o modelo ASEC-Rel prevê 3,3 ppm. Tomando a diferença $\sigma$ (ASEC-Rig) $-\sigma$ (ASEC-Rel), obtemos apenas 1, 8 ppm. Como temos discutido [139, 194], o efeito da relaxação geométrica sobre a blindagem magnética nuclear é irrelevante.

Aferimos as contribuições do solvente explícito considerando configurações em que o soluto, eletronicamente polarizado, interage com as moléculas do solvente através de ligações de hidrogênio. Esse modelo é referenciado no texto como LH e prediz uma blindagem de 10,9 9 1, 2 ppm. Note-se que esse modelo fornece uma descrição muito pobre do experimento. Para a blindagem magnética nuclear, tem sido sistematicamente mostrado que não basta incluir o solvente explícito nos cálculos de mecânica quântica [37, 45, 121, 139, 194]. $\mathrm{Na}$ verdade, as contribuições eletrostáticas das moléculas mais afastadas devem ser também consideradas. O modelo $\mathrm{LH}+\mathrm{CP}$, representa as configurações do modelo LH embebidas no campo eletrostático das 320 formamidas remanescentes representadas apenas por suas cargas eletrônicas. Esse modelo inclui todas as interações eletrostáticas até 15 A. Com essa aproximação obtivemos uma blindagem de 22, $9 \pm 1,0$ ppm, em excelente descrição do experimento.

Como no gás, a teoria do funcional da densidade não prediz resultados próximos ao experimento realizado em fase líquida, mas sua decrição do efeito do solvente $\Delta \sigma\left({ }^{17} \mathrm{O}\right)$ é consistente. Nosso melhor modelo $\mathrm{LH}+\mathrm{CP} / \mathrm{MP} 2 / 6-311++\mathrm{G}(d, p)$ prediz um efeito de solvente de 58,6 \pm 1,0 ppm. Usando o funcional B3LYP, obtivemos 66,7 $\pm 0,8 \mathrm{ppm}$. Fica claro que a influência do meio é dramática, neste âmbito, há razoável acordo nas descrições DFT e MP2.

\section{Revisitando modelos empíricos para solvatação em amidas}

Burgar, Amour, Fiat et al. (BAF) [213, 214] foram os primeiros a propor um modelo de solvatação que permitiu racionalizar e separar as diferentes contribuições do solvente sobre o espectro NMR em termos da hidrataçao de diferentes sítios moleculares em amidas. A 


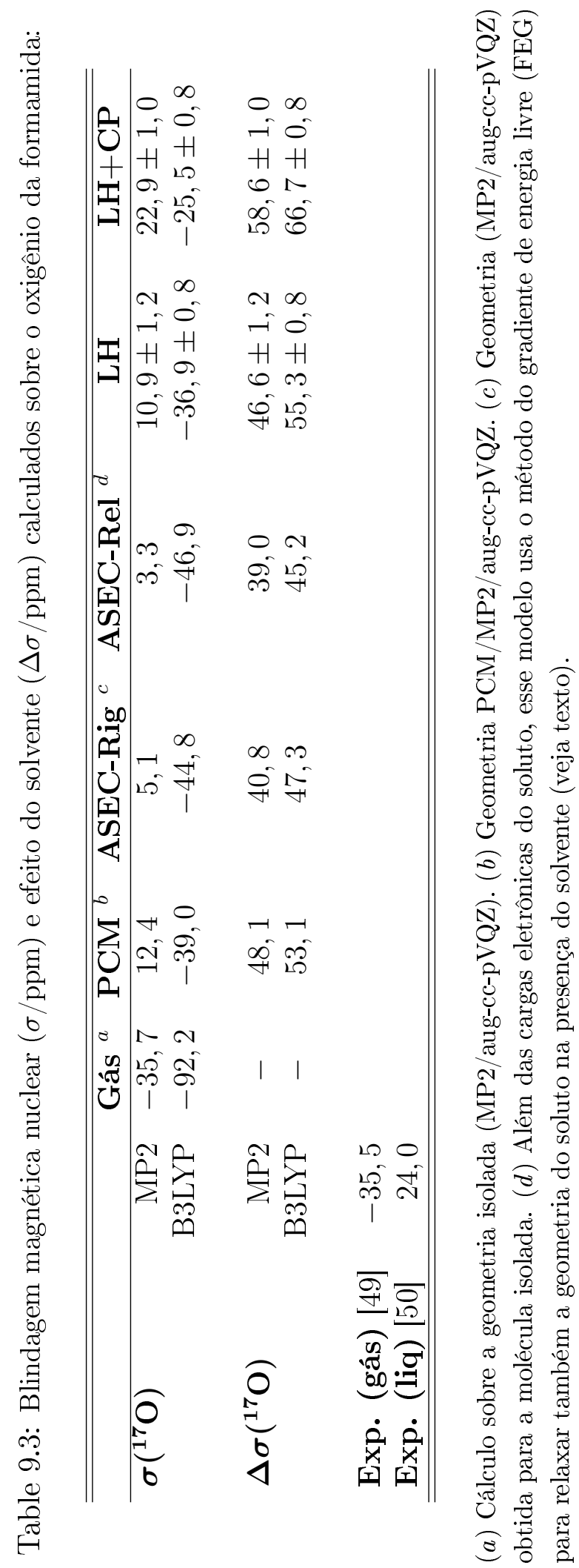


proposta consiste em desarrolar $\sigma\left({ }^{17} \mathrm{O}\right)$ nas componentes

$$
\sigma\left({ }^{17} \mathrm{O}\right)=\sigma_{\text {gás }}-\delta \mathrm{O}_{1}-\delta \mathrm{O}_{2}-\delta \mathrm{H}_{1}-\delta \mathrm{H}_{2}
$$

$\sigma_{\text {gás }}$ representa a blindagem magnética nuclear obtida para o gás. $\delta \mathrm{O}_{1}$ e $\delta \mathrm{O}_{2}$ representam as contribuições das ligações de hidrogênio sobre os lone pairs do oxigênio no soluto. Da mesma forma, $\delta \mathrm{H}_{1}$ e $\delta \mathrm{H}_{2}$, discriminam respectivamente as ligações de hidrogênio envolvendo os hidrogênios $\mathrm{H}_{1}$ e $\mathrm{H}_{2}$ do soluto. A figura 9.1 ilustra essas contribuições. Esse modelo se solvatação assume que essas componentes são independentes e obtidos em separado. Em particular, observe-se que $\delta \mathrm{O}_{1}, \delta \mathrm{O}_{2}, \delta \mathrm{H}_{1}$ e $\delta \mathrm{H}_{2}$ desvios tomados em relação à blindagem do gás, $\sigma_{\text {gás }}$.

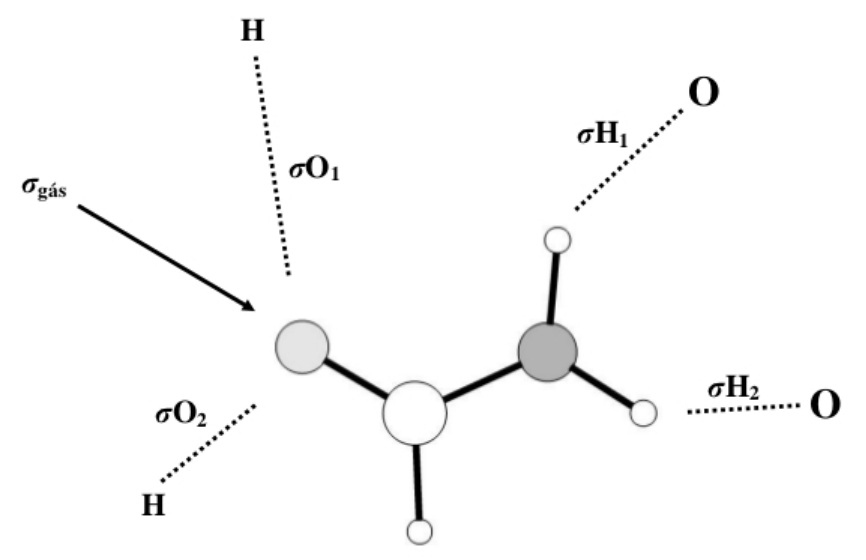

Figura 9.1: Modelo de solvatação em amidas. $\sigma_{\text {gás }}$ representa a blindagem magnética em formamida isolada. $\sigma \mathrm{O}_{1}$ e $\sigma \mathrm{O}_{2}$ são as contribuições devidas aos lone pairs do átomo de oxigênio envolvido nas ligações de hidrogênio. $\sigma \mathrm{H}_{1}$ e $\sigma \mathrm{H}_{2}$ são as contribuições devidas à solvatação dos hidrogênio do grupo amida.

Os parâmetros solvatocrômicos Kamlet-Abboud-Taft (KAT) também têm sido usados com frequência na descrição das propriedades magnéticas nucleares de elementos como o nitrogênio-14 [13, 49, 10, 9, 11, 12], por exemplo. No esquema KAT, a blindagem magnética total sobre um elemento de um dado soluto $\mathrm{X}$ em um dado solvente $\mathrm{Y}$ é descrita como

$$
\sigma^{\mathrm{X}}=\sigma_{\mathrm{CH}}^{\mathrm{X}}-s^{\mathrm{X}}\left(\pi_{\mathrm{Y}}^{*}+d^{\mathrm{X}} \delta_{\mathrm{Y}}\right)-a^{\mathrm{X}} \alpha_{\mathrm{Y}}-b^{\mathrm{X}} \beta_{\mathrm{Y}},
$$

onde os efeitos do meio são descritos pelos parêmetros $\pi_{\mathrm{Y}}^{*}, \delta_{\mathrm{Y}}, \alpha_{\mathrm{Y}}$ e $\beta_{\mathrm{Y}}$ que caracterizam o solvente. Os termos $a^{\mathrm{X}} \alpha_{\mathrm{Y}}$ e $b^{\mathrm{X}} \beta_{\mathrm{Y}}$ estão relacionados com as contribuições da formação de ligações de hidrogênio no oxigênio da carbonila e sobre os hidrogênios do grupo amida, 
respectivamente. Estes termos estão relacionados com o modelo BAF como

$$
a^{\mathrm{X}} \alpha_{\mathrm{Y}}=\delta \mathrm{O}_{1}+\delta \mathrm{O}_{2}
$$

e

$$
b^{\mathrm{X}} \beta_{\mathrm{Y}}=\delta \mathrm{H}_{1}+\delta \mathrm{H}_{2}
$$

Os modelos empíricos BAF e KAT são contraditórios principalmente quando ao papel da solvatação do grupo amida, tornando insertas suas contribuições sobre a blindagem magnética no oxigênio-17 [49]. Por meio de um modelo de aglomerados otimizados, Gerothanassis e Vaaka (GV) [17, 49, 55, 56] partiram da proposta empírica de BAF para explicar o papel do solvente sobre $\sigma\left({ }^{17} \mathrm{O}\right)$. Contudo, um líquido tem caráter estatístico, pois não há uma configuração representativa. Segundo a hipótese hergódica, todas as configurações do espaço de fases serão acessadas pelo sistema.

Para construir um modelo teórico mais realístico, utilizamos as estruturas MC geradas em nossas simulações clássicas e consideramos, em separado, a formação de ligações de hidrogênio sobre os sítios de interesse. Em seguida, calculamos, em nível MP2/6$311++\mathrm{G}(d, p)$, as contribuições $\delta \mathrm{O}, \delta \mathrm{H}_{1}$ e $\delta \mathrm{H}_{2}$ como médias tomadas sobre todas as configurações MC. A tabela 9.4 encerra os resultados obtidos com o tratamento s-MC/MQ em formamida hidratada, bem como outros dados encontrados na literatura. Após hidratar apenas o oxigênio do grupo carbonila considerando ligações de hidrogênio formadas apenas sobre esse sítio, obtivemos uma contribuição média de $\delta \mathrm{O}=-31,8 \pm 1,1 \mathrm{ppm}$. Este valor está em razoável acordo com a estimativa de -49,2 ppm obtida com os parâmetros KAT. Contudo, as contribuições $-61,6$ e -53 ppm, respectivamente estimadas pelos modelos GV e BAF são bem maiores. Isso ocorre porque estes modelos decompõe $\delta \mathrm{O}$ em duas componentes arbitrárias, $\delta \mathrm{O}_{1}$ e $\delta \mathrm{O}_{2}$, onde ambas possuem valores da mesma magnitude do valor que obtivemos com a aproximação s-MC/MQ. Em resumo, nossos resultados indicam BAF e GV podem estar considerando duas vezes a mesma contribuição.

Ao hidratar os hidrogênios do grupo amida, obtivemos contribuições de $\delta \mathrm{H}_{1}=-9,8 \pm$ 0,2 e $\delta \mathrm{H}_{2}=-9,5 \pm 0,5 \mathrm{ppm}$, respectivamente. Estes resultados corroboram a afirmativa de Gerothanassis e Vakka de que contribuições provenientes da hidratação do grupo amida são relevantes, ao contrário do que afirma o modelo empírico KAT. Note-se ainda que as predições s-MC/QM e $\mathrm{GV}$ indicam que $\delta \mathrm{H}_{1}$ e $\delta \mathrm{H}_{2}$ têm sinais opostos aos resultados preditos pelo modelo BAF.

Para o líquido homogêneo, obtivemos contribuições de $\delta \mathrm{O}=-16,9 \pm 0,5 \mathrm{ppm}, \delta \mathrm{H}_{1}=$ $-14,9 \pm 0,6 \mathrm{ppm}$ e $\delta \mathrm{H}_{2}=-12,7 \pm 0,3 \mathrm{ppm}$. Ao substituir esses valores na equação 9.1, obtêm-se uma blindagem magnética de 8,8 ppm, em boa concordância com o valor calculado utilizando o modelo LH, como disposto na tebela 9.3. Esta concorância significa 
Tabela 9.4: Valores calculados [MP2/6-311++G(d,p)] e empíricos para as contribuições da hidratação do oxigênio da carbonila e dos hidrogênio do grupo amida:

\begin{tabular}{ccccccc}
\hline \hline Contribuições & \multicolumn{2}{c}{ FA líquida } & \multicolumn{4}{c}{ FA em água } \\
& s-MC/QM & KAT $^{a}$ & s-MC/QM & GV $^{b}$ & BAF $^{c}$ & KAT $^{a}$ \\
\hline \hline$\delta \mathrm{O}_{1}+\delta \mathrm{O}_{2}$ & $-16,9 \pm 0,5$ & $-29,8$ & $-31,8 \pm 1,1$ & $-61,6$ & -53 & $-49,2$ \\
$\delta \mathrm{O}_{1}$ & - & - & - & $-33,1$ & -22 & - \\
$\delta \mathrm{O}_{2}$ & - & - & - & $-28,5$ & -31 & - \\
& & & & & & \\
$\delta \mathrm{H}_{1}+\delta \mathrm{H}_{2}$ & $-27,6$ & $-5,6$ & $-19,3$ & $-20,2$ & 11 & $-1,7$ \\
$\delta \mathrm{H}_{1}$ & $-14,9 \pm 0,6$ & - & $-9,8 \pm 0,2$ & $-15,3$ & 9 & - \\
$\delta \mathrm{H}_{2}$ & $-12,7 \pm 0,3$ & - & $-9,5 \pm 0,5$ & $-4,9$ & 2 & - \\
$\delta \mathrm{O}_{1}+\delta \mathrm{O}_{2}+\delta \mathrm{H}_{1}+\delta \mathrm{H}_{2}$ & $-44,5$ & $-35,4$ & $-51,1$ & $-81,8$ & -64 & $-50,9$ \\
\hline \hline
\end{tabular}

(a) Valores obtidos usando os parâmetros solvatocrômicos Kamlet-Abboud-Taft (KAT) [49]. (b) Designa o modelo de aglomerados proposto por Gerthanassis e Vaak [16, 49]. (c) Modelo empírico proposto Bougar-Amoud- Fiat et al. [51, 52].

que é coerente descrever as blindagem magnética total, $\sigma\left({ }^{17} \mathrm{O}\right)$, em termos da solvatação de outras partes da molécula de referência.

\section{Blindagem magnética $\sigma\left({ }^{15} \mathbf{N}\right)$}

Discutiremos agora o efeito do solvente sobre $\sigma\left({ }^{15} \mathrm{~N}\right)$. Todos os resultados estão organizados na tabela 9.5. Faremos uma digressão, principalmente em termos dos resultados MP2/6-311 $++(d, p)$. Não há dados experimentais para a blindagem magnética sobre o ${ }^{15} \mathrm{~N}$ em formamida isolada. Contudo, estimamos os valores 173,1 ppm. Para o líquido, Alkorta e Elguero têm reportado uma blindagem de 149,2 ppm [50]. O modelo contínuo polarizado prediz 166, 5 ppm para o líquido homogêneo. Essa predição difere consideravelmente do experimento por 17,3 ppm. Os modelos iterativos ASEC-Rig e ASEC-Rel não trabalham melhor. Com essas aproximações obtivemos blindagens de 165, 3 e 164, 5 ppm, respectivamente.

Interações explícitas soluto-solvente foram incluídas considerando as ligações de hidrogênio formadas entre soluto e solvente. Obtivemos uma blindagem de 150,1 ppm, em boa aproximação com a observação experimental. Novamente acoplamos interações com o solvente explícito à interações eletrostáticas provenientes das moléculas mais afastadas, representando-as apenas por suas cargas eletrônicas. Esse modelo mais realístico, rotu-

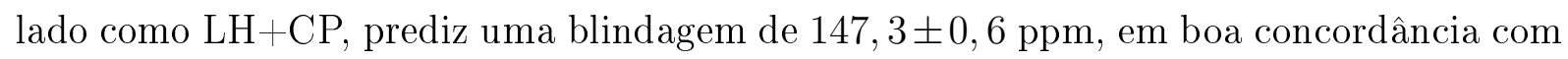
o experimento. 


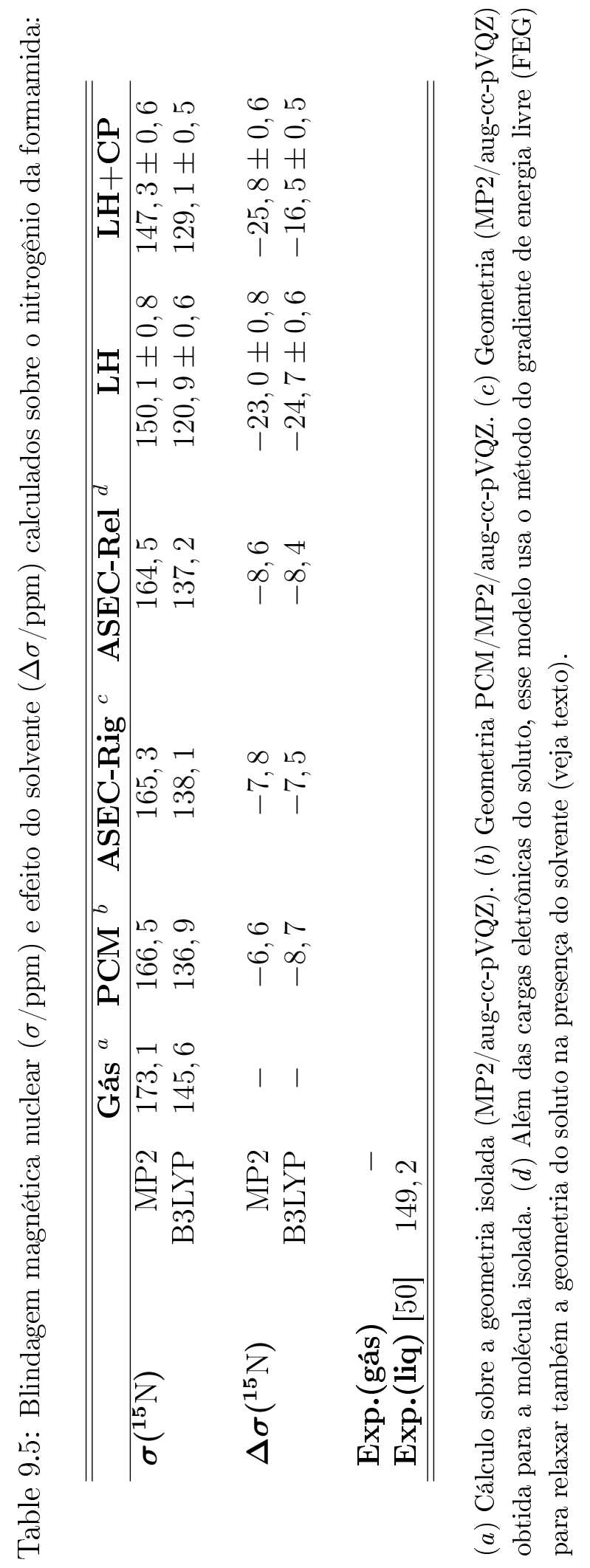


Trabalhos recentes $[9,10,11,12,13,14,15,45,121,139,194]$ mostram que o nitrogênio é tão sensível ao meio quanto outros elementos mais eletronegativos, como o oxigênio. Nosso melhor modelo, por exemplo, estima um considerável desvio gás-líquido de $-25,8 \pm 0,6 \mathrm{ppm}$. Para a blindagem magnética $\sigma\left({ }^{15} \mathrm{~N}\right)$, a teoria do funcional da densidade fornece resultados muito distantes do experimento. Contudo, novamente trabalha bem ao descrever o shift $\Delta \sigma\left({ }^{15} \mathrm{~N}\right)$ obtido com a aproximação perturbativa. Obtivemos com o modelo B3LYP $/ 6-311++\mathrm{G}(d, p)$ valores entre $-24,7 \pm 0,6$ e $-16,5 \pm 0,5$ ppm.

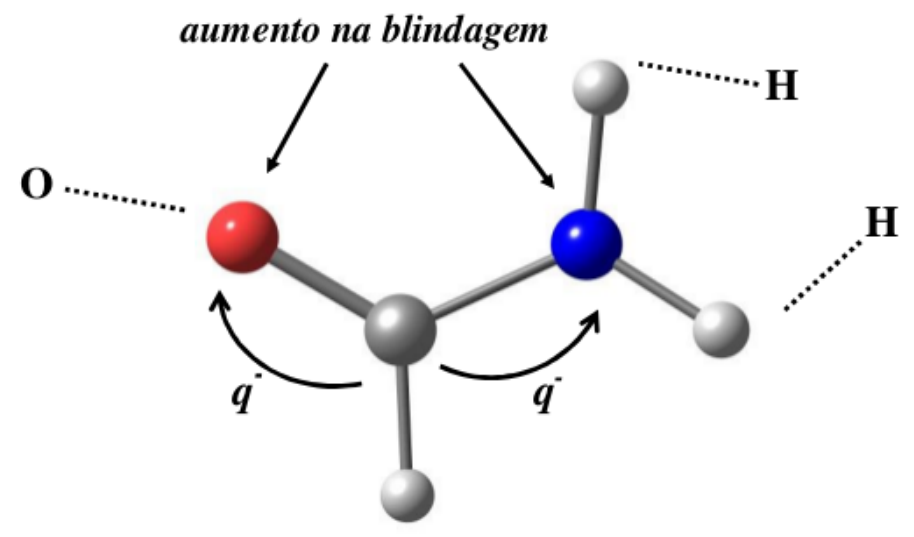

Figura 9.2: Modelo proposto (veja texto) para explicar o aumento das blindagens magnéticas nucleares $\sigma\left({ }^{15} \mathrm{~N}\right)$ e $\sigma\left({ }^{17} \mathrm{O}\right)$ em formamida solvatada. A migração das cargas eletrônicas é motivada pela presença do solvente. Quanto maior a polaridade do meio, maior é a transferência intramolecular de cargas, gerando um efeito de shielding (aumento na blindagem).

Uma discussão interessante concerne o sinal negativo do desvio $\Delta \sigma\left({ }^{15} \mathrm{~N}\right)$. Em termos da migração eletrônica intramolecular, Witanowski e colaboradores têm proposto um modelo plausível para explicar a blindagem magnética nuclear $\sigma\left({ }^{15} \mathrm{~N}\right)$ em amidas, aminas, uréias $[11,12,13,14]$, bem com em outros compostos nitrogenados. A figura 9.2 ilustra esse mecanismo. Segundo Witanowski, a polaridade do solvente motiva a migração de cargas eletrônicas no soluto. Quanto maior a polaridade do solvente, mais intensa será a migração eletrônica em direção a elementos eletronegativos, como o nitrogênio e oxigênio em formamida. No líquido, a população eletrônica sobre os elementos ${ }^{17} \mathrm{O}$ e ${ }^{15} \mathrm{~N}$ realmente sofre um acréscimo em relação à molécula isolada. Espera-se então, um aumento nas blindagens magnéticas $\sigma\left({ }^{17} \mathrm{O}\right)$ e $\sigma\left({ }^{15} \mathrm{~N}\right)$. Contudo, a resposta obtida para $\sigma\left({ }^{15} \mathrm{~N}\right)$ é anômala ao modelo idealizado por Wianoviski. A figura 9.3 torna evidente esse efeito mostrando a relação entre a blindagem e a redistribuição eletrônica sobre o nitrogênio em cada passo do processo iterativo. Está claro que ao ponto em que a carga eletrônica acumulada no sítio aumenta, a blindagem magnética decai. 


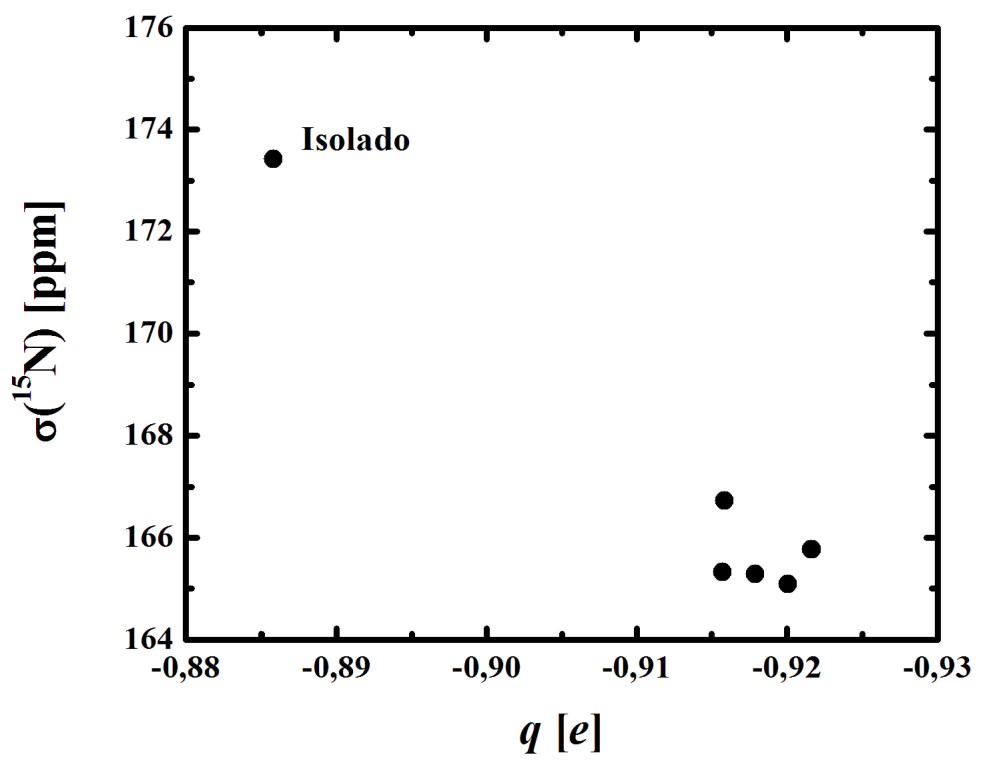

Figura 9.3: Relação entre blindagem magnética nuclear $\sigma\left({ }^{15} \mathrm{~N}\right)$ e a carga eletrônica $q$ calculadas em cada passo do procedimento iterativo. Observe que a presença do solvente diminui a blindagem magnética sobre o núcleo de interesse ao ponto que a população eletrônica sobre o mesmo aumenta.

Constatamos que a proposta de Witanowski é coerente ao explicar a blindagem magnética $\sigma\left({ }^{15} \mathrm{~N}\right)$ em uma série de compostos nitrogenados [45, 121, 194]. Em todos esses sistemas, o nitrogênio é o sítio aceitador na formação de ligações de hidrogênio. Em amônia líquida, entretanto, onde há também caráter prótico, embora a população eletrônica sobre o nitrogênio seja acrescida em relação ao gás, $\Delta \sigma\left({ }^{15} \mathrm{~N}\right)$ é negativo [139]. Uma explicação pode estar na natureza do esquema CHELPG.

Há, basicamente, três grandes metodologias utilizadas para relacionar cargas atômicas a um dado átomo. A primeira é composta por métodos que particionam as funções de onda em termos das funções base utilizadas. Deve-se evitar esse procedimento se é buscada a independência com respeito às funções bases empregadas, além do que, essa análise populacional de cargas não reproduz os momentos de dipolo e quadrupolo originais [19]. O segundo grupo, corresponde à metodologias baseadas em ajustes eletrostáticos, como o procedimento CHELPG [103], por exemplo. Esses esquemas são particularmente úteis em simulações computacionais clássicas, nas quais é fundamental obter um campo de forças consistente para reproduzir potencial eletrostático do soluto. Note-se que esses métodos comprometem-se em reproduzir o campo elétrico experimentado pelo solvente, mas a descrição da densidade eletrônica sobre um átomo, não é, necessariamente realística. No terceiro e último grupo encerram-se metodologias que buscam particionar a densidade eletrônica em termos dos domínios atômicos. Entre esses métodos encontram-se as apro- 
ximações NAO (natural atomic orbitals), NHO (natural hybrid orbitals), NBO (natural bond orbitals) e NLMO (natural localized orbitals), os quais foram pensados para mapear a densidade eletrônica em átomos e nas ligações entre eles [19]. Dada a filosofia dos métodos discutidos acima, investigamos o processo de polarização da formamida, agora hidratada, utilizando a população eletrônica NBO [225] e o ajuste eletrostático CHELPG [103], como implementado no código Gaussian 03 [118]. A escolha da água como solvente é estratégica, uma vez que alta polaridade tende a potencializar os efeitos da redistribuição de cargas no soluto.

O momento de dipolo calculado (MP2/aug-cc-pVQZ) obtida para a formamida hidratada usando o esquema CHELPG é 6,3 D, o que representa um considerável efeito de polarização, se comparado ao dipolo teórico obtido em vácuo de $3,9 \mathrm{D}$. Ao utilizar a aproximação NBO, obtivemos, em vácuo, um dipolo 4,6 D, ligeiramente maior que a precisão CHELPG. Em água, utilizando o procedimento iterativo, calculamos o dipolo de 6, 6 D, em boa concordância com o valor obtido iteraticamente utilizando o ajuste eletrostático CHELPG. Do ponto de vista do cálculos dos momentos dipolares da molécula de referência, nossos dados mostram ser indiferente obter a densidade eletrônica sobre os sítios de interesse utilizando ajustes eletrostáticos do potencial quântico, ou métodos que particionam a densidade eletrônica em termos dos orbitais atômicos.

Em formamida hidratada, discutiremos novamente a relação entre blindagem e carga eletrônica sobre o nitrogênio-15. A figuras 9.4 e 9.5 correlacionam essas duas propriedades em cada etapa dos ciclos de polarização iterativa realizados com o auxílio do esquema CHELPG e NBO, respectivamente. Ao utilizar o ajuste eletrostático CHELPG (feja figura 9.4), obtem-se um desvio gás-água de $-22,5 \mathrm{ppm}$. Note-se ainda que, ao aumento da carga eletrônica, correlaciona-se um decréscimo da blindagem magnética $\sigma\left({ }^{15} \mathbf{N}\right)$, contrariando o modelo de empírico de Witanowski [11, 12, 13, 14]. A polarização NBO (veja figura 9.5), por outro lado, prediz um efeito do solvente de $\Delta \sigma=-22,4$ ppm, correlacionando corretamente a blindagem às cargas eletrônicas, ou seja, a diminuição da blindagem magnética, necessariamente é demandada pela queda na população eletrônica sobre o sítio.

Apenas para ajudar a entender como o solvente atua sobre o mecanismo da blindagem magnética $\sigma\left({ }^{15} \mathrm{~N}\right)$, a figura 9.6 plota as curvas de nível da densidade de blindagem magnética nuclear (DBMN) calculadas sobre os nitrogênios da formamida isolada e na posição 9 do dímero. Note-se que a presença do solvente formando uma ligação de hidrogênio junto ao grupo amida, promove uma competição entre as superfícies positiva (em azul) e negativa (em vermelho) justamente sobre o $\mathrm{N}_{9}$, tornando-o menos blindado em relação à formamida isolada. 


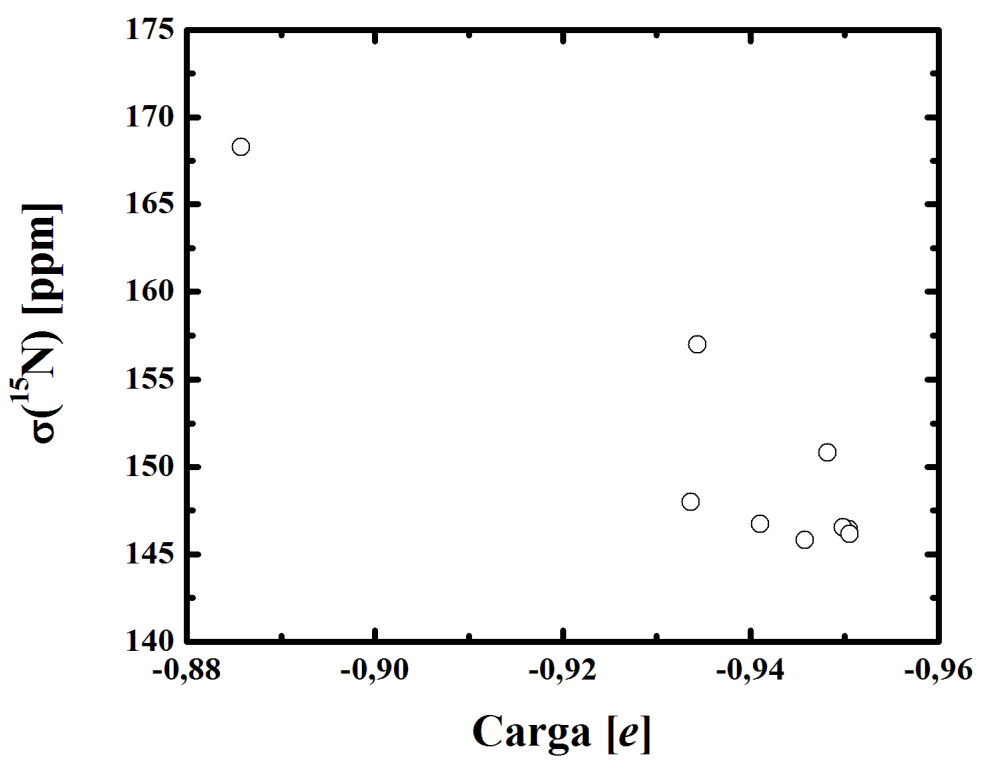

Figura 9.4: Relação entre blindagem magnética nuclear $\sigma\left({ }^{15} \mathbf{N}\right)$ e a carga eletrônica $q$ calculadas em cada passo do procedimento iterativo em água e usando o ajuste eletrostático CHELPG.

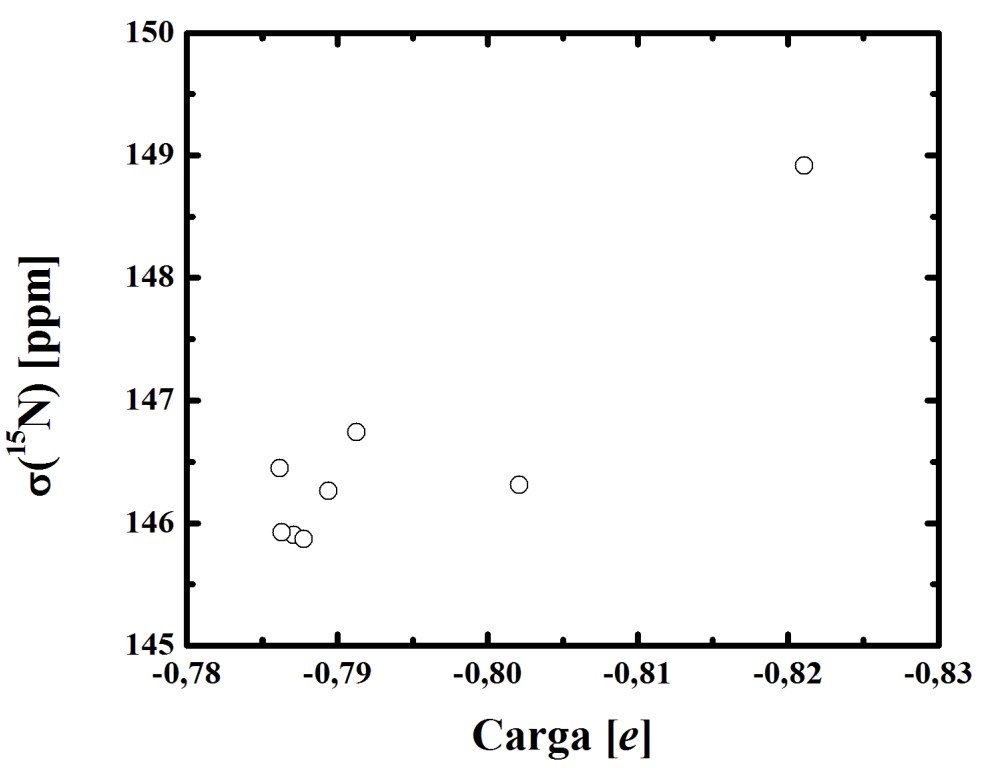

Figura 9.5: Relação entre blindagem magnética nuclear $\sigma\left({ }^{15} \mathrm{~N}\right)$ e a população eletrônica calculadas em cada passo do procedimento iterativo dentro do esquema NBO (natural bond orbital). Observe que a presença do solvente diminui a blindagem magnética sobre o núcleo de interesse ao ponto que a população eletrônica sobre o mesmo aumenta. 


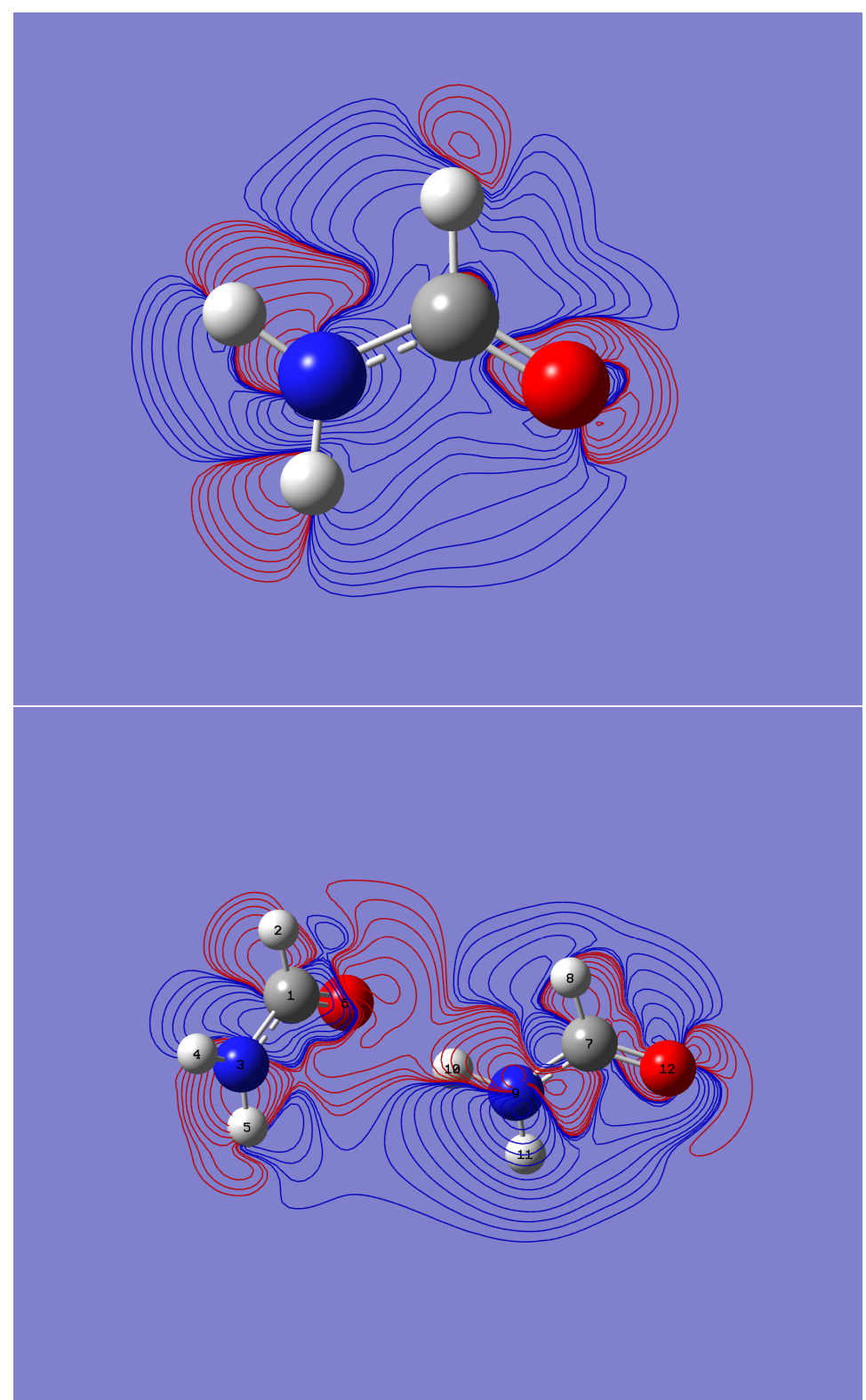

Figura 9.6: Curva de nível da densidade de blindagem magnética nuclear (DBMN). No dímero, as superfícies competem tornando o nitrogênio na posição 9 menos blindado quando comparado à formamida isolado acima.

\subsection{Conclusões}

Utilizamos um procedimento s-QM/MM para investigar os efeitos do solvente sobre as constantes magnéticas nucleares em formamida líquida. Dispensamos especial atenção sobre o papel da polarização eletrônica devido ao meio. Nesse âmbito, utilizamos vários modelos de solvente para aferir as interações soluto-solvente.

Calculamos (MP2/aug-cc-pVQZ) um momento de dipolo de 3,9 D para a molécula isolada. Resultado em excelente concordância com valor experimental de 3, 7 D observado 
para o gás. Embora não haja resultados para o líquido, nossos cálculos estimam para o líquido um dipolo variando entre 4,8 e 5,1 D. Nossas estimativas indicam uma considerável polarização em torno de $30 \%$.

A redistribuição das cargas eletrônicas no soluto tem efeitos drásticos sobre a estrutura do líquido. Utilizamos um procedimento iterativo que permite aferir esses efeitos comparando duas situações distintas. O primeiro e último estágios desse ciclo correspondem, respectivamente, a situações de polarização nula e total. Para o caso de polarização nula, observamos que a formação de ligações de hidrogênio soluto-solvente é mais frequênte para os casos em que uma (18\%), duas (35\%) e três (33\%) ligações são formadas. No caso polarizado, as mudanças no número de coordenação do solvente são drásticas. As ocorrências mais frequêntes correspondem à formação de três (40\%) e quatro (39,7\%) ligações de hidrogênio.

Nossos melhores resultados para blindagem magnética nuclear foram alcançados usando MP2 $/ 6-311++\mathrm{G}(d, p)$ como nível de cálculo. Calculamos uma blindagem de $-35,7$ ppm sobre isótopo ${ }^{17} \mathrm{O}$ em formamida isolada. Esse resultado está em excelente concordância com o valor experimental de $-35,4 \mathrm{ppm}$. Para o líquido, nossos melhores resultados foram obtidos combinando interações explícitas soluto-solvente à interações eletrostáticas. Neste modelo, estruturas soluto-solvente eletronicamente polarizadas, formando ligações de hidrogênio, são embebidas no campo eletrostático das moléculas remanescentes incluídas apenas como cargas pontuais. Obtivemos uma blindagem $\sigma\left({ }^{17} \mathrm{O}\right)$ de $22,9 \mathrm{ppm}$, em excelente descrição do valor experimental de 24,0 ppm.

O fato de a blindagem magnética nuclear ser uma propriedade local gera questões fundamentais. Uma dessas preocupações consiste em descrever a blindagem como a soma de contribuições independentes provenientes de interações específicas sobre determinados sítios moleculares. Para o caso da formamida, esses sítios seriam os hidrogênios do grupo amida e os lone pairs do oxigênio. Mostramos sistematicamente que a proposta é factível, desde que a polarização eletrônica do soluto, contribuições eletrostáticas provenientes do bulk e, principalmente, o caráter estatístico do líquido, sejam preservados. Ainda sobre a blindagem $\sigma\left({ }^{17} \mathrm{O}\right)$, um dos pontos fundamentais discute o papel das contribuições da solvatação do grupo amida. Mostramos sistematicamente que essas contribuições são expressivas, da ordem de $-20,0 \mathrm{ppm}$, contrariando o que prega a teoria atual.

Investigamos também a blindagem magnética sobre o isótopo ${ }^{15} \mathrm{~N}$. Para o líquido homogêneo, uma blindagem de 149,2 ppm tem sido reportada. Nossa melhor predição novamente combina estruturas soluto-solvente eletronicamente polarizadas à interações eletrostáticas provenientes das moléculas mais afastadas. Obtivemos com essa aproximação uma blindagem de 147,3 ppm, em excelente concordância com o experimento. Não há resultados em formamida isolada, mas estimamos uma blindagem de 173,1 ppm para 
o gás.

Embora as predições mais fiéis ao experimento tenham sido obtidas utilizando aproximações perturbativas, constatamos que a teoria do funcional da densidade reproduz muito bem o desvio gás-líquido. Para o oxigênio, por exemplo, obtivemos um desvio de 66,7 ppm, em boa concordância com os valores perturbativo $(58,6 \mathrm{ppm})$ e experimental $(59,5$ ppm). Para o nitrogênio, a DFT prediz -16,5 ppm como efeito do solvente. Novamente, em boa concordância com a predição perturbativa.

Outra questão fundamental versa sobre a origem do sinal negativo do shift $\Delta \sigma\left({ }^{15} \mathrm{~N}\right)$. Uma das propostas vigentes discute que o acúmulo de cargas eletrônicas sobre um dado núcleo é causado pela migração das cargas eletrônicas devido à polaridade do meio. Um núcleo com uma maior densidade eletrônica estaria, em tese, mais blindado magneticamente. Formamida líquida não contraria essa perspectiva, mas do ponto de vista computacional, essa característica apenas é confirmada utilizando métodos mais sofisticados para calcular a população eletrônica, como NBO, por exemplo. Mostramos sistematicamente que qualquer interação intermolecular é capaz de reproduzir essa tendência, mas ligações de hidrogênio sobre o grupo amida potencializam esse efeito. A presença do próton na ponte de hidrogênio ( $\mathrm{NH} \cdots \mathrm{O}$ ) causa uma queda na blindagem $\sigma\left({ }^{15} \mathrm{~N}\right)$, justificando o sinal negativo em $\Delta \sigma\left({ }^{15} \mathrm{~N}\right)$. Em outras palavras, nossos resultados indicam que para entender esse efeito é necessário considerar interações específicas soluto-solvente, como ligações de hidrogênio. 


\section{Capítulo 10}

\section{Conclusões finais e perspectivas}

Um tratamento QM/MM sequencial foi utilizado para investigar propriedades magnéticas nucleares em líquidos moleculares. Há particular interesse na blindagem magnética nuclear e no acoplamento indireto entre spins nucleares, uma vez que essas constantes, juntas, proporcionam uma análise completa do espectro RMN, dando informações precisas sobre a estrutura molecular e ambiente químico.

Os objetivos principais resumem-se em, primeiro, entender quais interações entre soluto e solvente são fundamentais às propriedades moleculares de interesse. Segundo, desenvolver metodologias eficientes para aferir os efeitos do solvente sobre o espectro RMN. Dentre os isótopos mais estudos em RMN, figuram o ${ }^{1} \mathrm{H}$ e ${ }^{13} \mathrm{C}$, contudo muitos sistemas moleculares de interesse possuem elementos, como o ${ }^{15} \mathrm{~N}$ e ${ }^{17} \mathrm{O}$. Esses isótopos têm particular importância em RMN devido a uma série de fatores. Normalmente ocupam posições estratégicas na molécula, além de estarem diretamente envolvidos em interações intermoleculares específicas, como por ligações de hidrogênio, por exemplo. Essas características tornam de grande interesse os espectros RMN desses elementos. A fim de viabilizar os objetivos, os sistemas investigados foram estrategicamente escolhidos considerando (1) seu caráter prótico ou aprótico, (2) interesse biológico e químico e (3) hibridização dos orbitais $s$ e $p$.

Com respeito ao papel das interações intermoleculares sobre propriedades magnéticas em líquidos, há consenso sobre duas contribuições fundamentais. A primeira provem de interações específicas, como ligações de hidrogênio formadas sobre o sítio molecular de interesse. A segunda tem origem nas interações de longo alcance provenientes das moléculas mais afastadas. Entre essas últimas, destacam-se interações eletrostáticas. Contudo, há indícios de que a polarização eletrônica sobre o soluto, devido ao meio, tem papel fundamental sobre as constantes magnéticas nucleares.

Utilizamos vários modelos contínuos e discretos de solvente para inferir aferir os efeitos da polarização eletrônica sobre os parâmetros RMN de interesse. Obtivemos em for- 
Tabela 10.1: Resumo dos sistema moleculares estudados. Mostra-se o sentido da variação das cargas eletrônicas $\Delta q_{\mathrm{X}}$ calculadas sobre o isótopo $\mathrm{X}$, bem como os desvios gás-líquido $\Delta \sigma_{\mathrm{Y}}{ }^{a}$ obtidos utilizando diferentes modelos de solvente:

\begin{tabular}{|c|c|c|c|}
\hline Piridina & Pirimidina & Uracila & Formamida \\
\hline$\Delta q_{\mathrm{N}}>0$ & $\Delta q_{\mathrm{N}}>0$ & $\Delta q_{\mathrm{O}}>0$ & $\Delta q_{\mathrm{O}}>0$ \\
\hline $\begin{array}{l}\Delta \sigma_{\mathrm{LHCP}}=41 \\
\Delta \sigma_{\mathrm{ASEC}}=43 \\
\Delta \sigma_{\mathrm{PCM}}=21,8\end{array}$ & $\begin{aligned} \Delta \sigma_{\mathrm{LHCP}} & =24,5 \\
\Delta \sigma_{\mathrm{ASEC}} & =25,8\end{aligned}$ & $\begin{array}{l}\Delta \sigma_{\mathrm{LHCP}}=103,1 \\
\Delta \sigma_{\mathrm{ASEC}}=100 \\
\Delta \sigma_{\mathrm{PCM}}=72\end{array}$ & $\begin{array}{l}\Delta \sigma_{\mathrm{LHCP}}=58,6 \\
\Delta \sigma_{\mathrm{ASEC}}=40,8 \\
\Delta \sigma_{\mathrm{PCM}}=48,1\end{array}$ \\
\hline Formamida & Amônia & Metilamina & Aminobenzeno \\
\hline$\Delta q_{\mathrm{N}}<0$ & $\Delta q_{\mathrm{N}}>0$ & $\Delta q_{\mathrm{N}}>0$ & $\Delta q_{\mathrm{N}}>0$ \\
\hline $\begin{array}{l}\Delta \sigma_{\mathrm{LHCP}}=-25,8 \\
\Delta \sigma_{\mathrm{ASEC}}=-7,8 \\
\Delta \sigma_{\mathrm{PCM}}=-6,6\end{array}$ & $\begin{array}{l}\Delta \sigma_{\mathrm{LHCP}}=-25,2 \\
\Delta \sigma_{\mathrm{ASEC}}=6,9 \\
\Delta \sigma_{\mathrm{PCM}}=3,0\end{array}$ & $\begin{array}{l}\Delta \sigma_{\mathrm{LHCP}}=-5,7 \\
\Delta \sigma_{\mathrm{ASEC}}=11,8 \\
\Delta \sigma_{\mathrm{PCM}}=3,3\end{array}$ & $\begin{array}{l}\Delta \sigma_{\mathrm{LHCP}}=-10,2 \\
\Delta \sigma_{\mathrm{ASEC}}=7,6 \\
\Delta \sigma_{\mathrm{PCM}}=07\end{array}$ \\
\hline
\end{tabular}

(a) O modelo LHCP indica que estruturas soluto-solvente fortemente ligadas através de ligações de hidrogênio são embebidas no campo elétrico dos demais constituintes do líquidos, estes considerados como cargar pontuais. (b) ASEC indica que o modelo da configuração eletrostática média do solvente foi utilizado. (c) PCM indica o modelo contínuo polarizado.

mamida líquida, por exemplo, um momento de dipolo variando entre 4,8 até 5,1 D. Estes valores representam, em média, um aumento de $23 \%$ em relação ao dipolo calculado (MP2/aug-cc-pVTZ) para a molécula isolada. Em piridina e pirimidina hidratas, o efeito da polarização é mais drástico, onde calculamos variações no momento de dipolo de até $69 \%$ e $75 \%$, o que mostra uma intensa redistribuição de cargas no soluto.

A polarização eletrônica tem efeitos dramáticos sobre a estrutura do líquido, reorganizando, por exemplo, a coordenação do solvente sobre o soluto. Como o espectro RMN está correlacionado aos rearranjos estruturais, espera-se, mesmo que minimamente, sensibilidade das propriedades magnéticas em relação à polarização eletrônica do soluto. 
Nossos cálculos confirmam essa característica. Todas as constantes magnéticas nucleares foram calculadas utilizando métodos ab initio (MP2 ou DFT), funções base apropriadas e o método GIAO. Nossos melhores resultados são obtidos acoplando interações explícitas soluto-solvente embebidas no campo elétrico das moléculas do solvente remanescentes consideradas apenas como cargas pontuais. Essas configurações foram obtidas administrando um procedimento iterativo que permite incluir os efeitos da polarização através do relaxamento das cargas eletrônicas do soluto na presença do solvente. Note-se que esse modelo permite descrever parte do solvente utilizando métodos de mecânica quântica e simultaneamente considera interações com bulk. Com esse modelo eletronicamente polarizado, obtivemos sobre um dos átomos de oxigênio da uracila hidratada, uma blindagem magnética de 55,3 ppm, em muito boa concordância com o valor experimental de 55,5 ppm. Descrições nesse nível de acurácia são uma constante ao longo do trabalho, o que torna indiscutível a necessidade de assegurar os efeitos da polarização eletrônica.

É razoável pensar que o acúmulo de cargas eletrônicas sobre um dado átomo implique no aumento de sua blindagem. Poucas técnicas experimentais, como a microscopia de tunelamento por varredura, são capazes de relacionar com eficácia a cargas eletrônicas a sítios atômicos. Por outro lado, métodos de modelagem molecular e aproximações de mecânica quântica podem fornecer com facilidade esse tipo informação. Em alguns dos sistemas moleculares discutidos nesta tese, observamos que variações na blindagem magnética realmente acompanham o sentido da variação da carga eletrônica acumulada no isótopo de interesse. Essa característica tem sido observada para os nitrogênios em piridina, pirimidina e formamida, bem como para os oxigênios em uracila, 5-fuorouracila e formamida, como disposto na tabela 10.1. Note-se que nesses casos o sinal de $\Delta \sigma$ correlaciona-se como esperado ao sinal de $\Delta q$, corroborando as idéias de Witanowski sobre a dependência da blindagem magnética nuclear quanto aos efeitos da redistribuição de cargas eletrônicas no soluto. Contudo, note-se que para os nitrogênios em amônia, metilamina e aminobenzeno, obtem-se outra tendência. Nestes sistemas, onde o nitrogênio apresenta hibridização $s p^{3}$, embora haja um aumento de cargas eletrônicas no isótopo de interesse, $\Delta \sigma_{\text {LHCP }}$ indica desvio negativos. Os resultados mostrados aqui indicam que variações na blindagem magnética não podem ser determinadas unicamente por variações nas cargas eletrônicas acumuladas em um dado isótopo. Note-se ainda a incapacidade dos modelos eletrostáticos ASEC e PCM na descrição dos sistemas com hidridização $s p^{3}$ considerados. Isso é explicado analisando a equação originalmente deduzida por Ramsey para a blindagem magnética nuclear

$$
\sigma=\frac{\alpha^{2}}{3} \sum_{j k}\left\langle 0\left|\mathbf{1} / r_{j k}\right| 0\right\rangle-\frac{2 \alpha^{2}}{3 \Delta \varepsilon} \sum_{j k}\left\langle 0\left|r_{j k}^{-3} \mathbf{L}_{j} \cdot \mathbf{L}_{j}\right| 0\right\rangle .
$$


O primeiro termo é descrito pela densidade eletrônica no isótopo de interesse, já o segundo depende explicitamente do momento angular eletrônico. Os modelos eletrostáticos de solvente negligenciam este último termo, já que o momento angular do solvente não é considerado. Em suma, as descrições obtidas com modelos eletrostáticas são governadas por variações na densidade eletrônica no isótopo de interesse, isso explica a falha desses modelos em descrever o nitrogênio $s p^{3}$.

Conclui-se que modelos eletrostáticos de solvente, sejam contínuos ou discretos, devem de ser usados com cuidado no estudo da blindagem magnética nuclear. O modo mais seguro de descrever essa propriedade magnética consiste em assegurar a presença do solvente mais fortemente ligado ao soluto, considerando as ligações de hidrogênio, por exemplo, e assegurar interações eletrostáticas de longo alcance com os remanescentes do líquido. Adicionalmente, os resultados obtidos para sistemas com hibrização $s p^{3}$, o que implica em uma geometria não planar, indicam que não é seguro racionalizar e descrever a blindagem magnética em termos da variação de cargas eletrônicas sobre um dado isótopo.

O real papel do solvente sobre a blindagem magnética nuclear é outro padrão percebido na tabela 10.1. Observa-se que desvios gás-líquido $\Delta \sigma>0$ são obtidos quando o isótopo estudado interage diretamente com o solvente como aceitador em uma ligação de hidrogênio. Contrariamente, quando o isótopo de interesse participa dessas interações como doador, desvios $\Delta \sigma>0$ são obtidos. Note-se ainda que esse efeito independe da carga eletrônica acumulada no átomo blindado. Para sistemas com hibridização $s p^{3}$ há um efeito competitivo, mas como mostramos em amônia líquida, predomina o caráter doador em LH.

Outros efeitos interessantes têm origem nas variações geométricas na molécula de referência causadas pela interação com o solvente e, ao longo do texto, discutimos essas contribuições. Para a blindagem magnética, por exemplo, observamos que esses efeitos têm pouca importância para os sistemas investigados, nos quais, obtivemos contribuições não maiores que 1,0 ppm. Contudo, outras propriedades, como o acoplamento indireto entre spins nucleares, são consideravelmente mais sensíveis aos rearranjos estruturais intramoleculares. Em amônia líquida, utilizando o método do gradiente de energia livre proposto por Nagaoka, obtivemos variações no acoplamento ${ }^{1} \mathrm{~J}(\mathrm{~N}-\mathrm{H})$ de até $3,0 \mathrm{~Hz}$. Tendo em vista a dependência do acoplamento entre spins nucleares quanto ao efeitos de relaxação geométrica, espera-se que simulações por dinâmica molecular clássica, ou utilizando os métodos ab initio de Car-Parrinello ou Born-Oppenheimer possam fornecer uma descrição ainda mais apurada dessa propriedade magnética.

Quanto à perspectivas, do ponto de vista de aplicações, há muitas alternativas ainda inexploradas pela ciência. Uma dessas recentes discussões concerne sobre o estudo de sistemas moleculares quirais. Em condições normais, a quiralidade é insensível à RMN 
[226]. Durante muitos anos, a determinação de enantiômeros em misturas quirais foi feita utilizando solventes quirais específicos, os quais têm a propriedade de converter o líquido em uma mistura estereoisomérica [226]. Recentemente, Buckingham [227] e Buckingham e Fischer [228] mostraram que o "problema" da quiralidade pode ser contornado em RMN submetendo o sistema molecular a um campo elétrico externo, perpendicular ao campo magnético. Essas descobertas recentes têm dado novos rumos à RMN.

Núcleos magnéticos não são as únicas partículas capazes de interagir com campos magnéticos e fornecer informações sobre a estrutura da matéria. Ressonância paramagnética eletrônica (RPE) é uma técnica cujos conceitos básicos são similares à RMN. Contudo, em RPE é o spin eletrônico quem sofre excitações. Como as moléculas mais estáveis têm seus elétrons emparelhados, sendo invisíveis à técnica, RPE tem sido menos utilizada que RMN. Todavia, respeitadas suas limitações, RPE tem aplicabilidade em vários ramos da ciência, como biologia, química e física, sendo utilizada na detecção de radicais livres e centros paramagnéticos. Ao considerar as novas aplicações para RMN, bem como as potencialidades de outras técnicas, como RPE, está clara a necessidade do investimento em mapear os efeitos do solvente sobre propriedades magnéticas, eletrônicas e nucleares. 


\section{Referências Bibliográficas}

[1] I.I. Rabi, J.R. Zacharias, S. Millman, P. Kusch, Physical Review, 53 (1938) 318.

[2] F. Bloch, W.W. Hansen e M.E. Packard, Physical Review, 69 (1946) 127.

[3] E.M. Purcell, H.C. Torrey e R.V. Pound, Physical Review, 69 (1946) 127.

[4] J.P. Grivet, A.M. Delort, Progress in Nuclear Magnetic Resonance Spectroscopy, 54 (2009) 1 .

[5] Macomber, R.S.; Complete Introduction to Nuclear Magnetic Resonance and NMR Spectroscopy. New York, John Wiley \& Sons, 1998.

[6] Blümich, B.; NMR Imaging of Materials, New York. Oxford, 2000.

[7] Huettel, S., Song, A.W., McCarthy, G.;, Functional Magnetic Resonance Imaging, Second Edition. Sinauer Associates, Massachussetts, 2004.

[8] Reichardt, C.; Solvents and Solvent Effects in Organic Chemistry, Third edition, New York: John Wiley \& Sons, 1988.

[9] M. Witanowski, W. Sicinska, S. Biernat, G.A. Webb, Journal of Magnetic Resonance, 91 ( 1991) 289.

[10] M. Witanowski, Z. Biedrzycka, W. Sicinska, Z. Grabowski, G.A. Webb, Journal of Magnetic Resonance, 124 (1997) 127.

[11] M. Witanowski, W. Sicinska, Z. Biedrzycka, G.A. Webb, Journal of Molecular Structure, 404 (1997) 267.

[12] M. Witanowski, Z. Biedrzycka, W. Sicinska, and Z. Grabowski, Journal of Magnetic Resonance, 131 (1998) 54.

[13] M. Witanowski, W. Sicinska, Z. Biedrzycka, G.A. Webb, Journal of Molecular Structure, 476 (1999) 133. 
[14] M. Witanowski, Z. Biedrzycka, W. Sicinska, Z. Grabowski, Journal of Magnetic Resonance, 164 (2003) 212.

[15] M. Witanowski, K. Kamienska-Trela, Z. Biedrzycka, M. Bechcicka, Journal of Physical Organic Chemistry, 21 (2008) 185.

[16] I.P. Gerothanassis, Progress in Nuclear Magnetic Resonance Spectroscopy, 56 (2010) 95.

[17] I.P. Gerothanassis, Progress in Nuclear Magnetic Resonance Spectroscopy, 57 (2010) 1.

[18] Cramer, C.J.; Essentials of Computational Chemistry Theories and Models, Second edtion, Chichester: John Wiley \& Sons, 2004.

[19] Jensen, F.; Introduction to Computational Chemistry. Second edition. Chichester: John Wiley and Sons, 2007.

[20] C.J. Cramer, D.G. Truhlar, Chemical Review, 99 (1999) 2161.

[21] F.J. Luque, C. Curutchet, J. Munoz-Muriedas, A. Bidon-Chanal, I. Soteras, A. Morreale, J.L. Gelpıb, M. Orozco, Physical Chemistry Chemical Physics, 5 (2003) 3827.

[22] M. Born, Zeitschrift für Physik, 1 (1920) 45.

[23] L. Onsager, Journal of American Chemical Society, 58 (1936) 1486.

[24] J.G. Kirkwood, Journal of Chemical Physics, 2 (1934) 351.

[25] S. Miertus, E. Scrocco, J. Tomasi, Chemical Physics, 55 (1981) 117.

[26] E. Cancès, B. Mennucci, J. Tomasi, Journal of Chemical Physics, 107 (1998) 253.

[27] B. Mennucci, Journal of Physics Chemical Letters, 1 (2010) 1666.

[28] J. Seltmann, A. Marini, B. Mennucci, S. Dey, S. Kumar, M. Lehmann, Chemistry of Materials, 23 (2011) 2630.

[29] M. Pavanello, B. Mennucci, A. Ferrarini, Journal of Chemical Physics, 122 (2005) 064906.

[30] B. Mennucci, R. Cammi, International Journal of Quantum Chemistry, 93 (2003) 121. 
[31] K. Coutinho, S. Canuto, Advances in Quantum Chemistry, 28 (1997) 89.

[32] K. Coutinho, S. Canuto, Journal of Molecular Structure: THEOCHEM, 632 (2003) 235.

[33] S. Canuto, K. Coutinho, International Journal of Quantum Chemistry, 77 (2000) 192.

[34] A.D. Buckingham, T. Schaefer, W.G. Schneider, Journal of Chemical Physics, 32 (1960) 1227.

[35] A.D. Buckingham, Canadian Journal of Chemistry, 38 (1960) 300.

[36] T.L. Fonseca, K. Coutinho, S. Canuto, Journal of Chemical Physics, 129 (2008) 034502.

[37] B. Mennucci, J.M. Martinez, J. Tomasi, Journal of Physical Chemistry A, 105 (2001) 7287.

[38] Solvation Effects on Molecules and Biomolecules. Computational Methods and Applications, ed. S. Canuto, Springer, 2008.

[39] Israelachvili, J.N.; Intermolecular and Surfaces Forces, California, Academic Press, 1991.

[40] J.D. Garrido, Y. Maikel; Y. Orozco-González, S. Canuto, Journal of Physical Chemistry A, 115 (2011) 1453.

[41] L. De Boni, D.S. Correa, D.L. Silva, P.J. Gonçalves, S.C. Zilio, G.G. Parra, I.E. Borissevitch, S. Canuto, C.R. Mendonca, Journal of Chemical Physics, 134 (2011) 014509.

[42] P. Jaramillo, K. Coutinho, Kaline, B.J.C. Cabral, S Canuto, Chemical Physics Letters, 516 (2011) 250.

[43] P. Jaramillo, K. Coutinho, S. Canuto, International Journal of Quantum Chemistry,110 (2010) 2371.

[44] V. Ludwig, K. Coutinho, S. Canuto, Physical Chemistry Chemical Physics, 9 ( 2007) 4907.

[45] V. Manzoni, M.L. Lyra, K. Coutinho, S. Canuto, Journal of Chemical Physics, 135 (2011) 144103. 
[46] R.C. Barreto, K. Coutinho, H.C. Georg, S. Canuto, Physical Chemistry Chemical Physics, 11 (2009) 1388.

[47] H.C. Georg, K. Coutinho, S. Canuto, Chemical Physics Letters, 429 (2006) 119.

[48] K. Coutinho, H.C. Georg, T.L. Fonseca, V. Ludwig, S. Canuto, Chemical Physics Letters, 437 (2007) 148.

[49] E. Dieaz, J. San Fabian, I.P. Gerthanassis, A.L. Esteban, J.L.M. Abboud, R.H. Contreras, D.G. Kowalewski, Journal of Magnetic Resonance, 124 (1997) 8 .

[50] I. Alkorta, J. Elguero, Structural Chemistry, 9 (1998) 187.

[51] M. I. Burgar, T. E. St. Amour, D. Fiat, J. Phys. Chem. 85 (1981) 502.

[52] B. Valentine, A. Steinschneider, D. Dhawan, M. I. Burgar, T. St. Amour, D. Fiat, Int. J. Pept. Protein Res. 25 (1985) 26.

[53] J.D.M. Vianna, A. Fazzio e S. Canuto, Teoria Quântica de Sólidos e Moléculas, Livraria da Física, São Paulo (2004).

[54] A. Szabo e N. S. Ostlund, Modern Quantum Chemistry - Introduction to Advanced Electronic Structure Theory, Dover, New York (1982).

[55] I.P. Gerothanassis, Progress in Nuclear Magnetic Resonance Spectroscopy, 26 (1994) 171.

[56] I.P. Gerothanassis, C. Vakka, Journal of Organic Chemistry, 59 (1994) 234.

[57] C.C.J. Roothaan, Reviews of Modern Physics, 23 (1951) 69.

[58] Levine, I.N.; Quantum Chemistry, Fifth edition, New jersey: Pearson Prentice Hall, 2009.

[59] P. Hohenberg, W. Kohn, Physical Review B, 136 (1964) 864.

[60] W. Kohn, L.J. Sham, Phys. Rev. A, 140 (1965) 1133.

[61] S.F. Boys, Proceedings of the Royal Society of London A, 200 (1950) 542.

[62] Atkins, P.; Friedman, R.; Molecular Quantum Chemistry. Sixth edition. New York: Oxford, 2011. 
[63] T.H. Dunning Jr., Journal of Chemical Physics, 90 (1989) 1007.

[64] R.A. Kendall, T.H. Dunning Jr., R.J. Harrison, Journal of Chemical Physics, 96 (1992) 1358.

[65] D.E. Woon, T.H. Dunning Jr., Journal of Chemical Physics, 98 (1993) 1358.

[66] R.O. Duthaler, J.D. Roberts, Journal of American Chemical Society, 100 (1978) 4969.

[67] P.F. Provasi, G.A. Aucar, S.P.A. Sauer, Journal of Chemical Physics, 115 (2001) 1324.

[68] The aug-cc-pVTZ-J basis sets can be downloaded from https://bse.pnl.gov/bse/portal.

[69] T.H. Dunning, Jr. Journal of Chemical Physics, 90 (1989) 1007.

[70] D.E. Woon, T.H. Dunning, Jr., Journal of Chemical Physics, 98 (1993) 1358.

[71] W.A. de Jong, R.J. Harrison,D.A. Dixon, Journal of Chemical Physics, 114 (2001) 48.

[72] N.B. Balabanov, K.A. Peterson, Journal of Chemical Physics, 123 (2005) 064107.

[73] D.E. Woon, T.H. Dunning, Jr., Journal of Chemical Physics, 98 (1993) 1358.

[74] A.K. Wilson, D.E. Woon, K.A. Peterson, T.H. Dunning, Jr., Journal of Chemical Physics, 110 (1999) 7667.

[75] K. Coutinho, S. Canuto, Advances in Quantum Chemistry, 28 (1997) 89.

[76] M.P. Allen, D.J. Tildesley, Computer Simulation of Liquids, Oxford University Press, Oxford (1987).

[77] Coutinho, K.R., Modelo Discreto de Solvente. Solvatocromismo no Espectro de Absorção Molecular, Tese (Doutorado), Instituto de Física da Universidade de São Paulo, 1997.

[78] N.M. Metropolis, A.W. Rosenbluth, A.N. Teller, E. Teller, Journal of Chemical Physics, 21 (1953) 1087. 
[79] J. Pranata, S.G. Wierschke, W.L. Jorgensen, Journal of the American Chemical Society, 113 (1991) 2810.

[80] W. Pauli, Naturwiss, 12 (1924) 741.

[81] N. F. Ramsey, Physical Review, 78 (1950) 699.

[82] Hameka, H.F., Advanced Quantum Chemistry, Theory of Interaction between Moleculaes and Electromagnetic Fields, Adson-Wesley, 1965.

[83] F. London, Journal of Physics Radium, 8 (1937) 397.

[84] R. Ditchfield, Molecular Physics, 27 (1974) 789.

[85] N.F. Ramsey, Physical Review, 91 (1953) 303.

[86] T. Helgaker, M. Jaszunski, K. Ruud, Chemical Review, 99 (1999) 293.

[87] C. Gutlé, J.Y. Salpin, T. Cartailler, J. Tortajada, M.P. Gaigeot, Journal of Physical Chemistry A, 110 (2006) 11684.

[88] A. Eizaguirre, A.M. Lamsabhi, O. Mó, M. Yáñez, Theoretical Chemistry Accounts, 128 (2011) 457.

[89] A. Golan, K.B. Bravaya, R. Kudirka, O. Kostko, S.R. Leone, A.I. Krylov, M. Ahmed, Nature Chemistry, 4 (2012) 323.

[90] A. DeFusco, J. Ivanic, M.W. Schmidt, M.S. Gordon, Journal of Physical Chemistry A, 115 (2011) 4574.

[91] D. Wang, Y. Su, C. Jin, B Zhu, Y. Pang, L. Zhu, J. Liu, C. Tu, D. Yan, X. Zhu, Biomacromolecules, 12 (2011) 1370.

[92] S.S. Sundar, D Sangeetha, Journal of Materials Science, 23 (2012) 1421.

[93] J.P. Kokko, L. Mandell, J.H. Goldstein, Journal of American Chemical Society, 84 (1962) 1042.

[94] P.D. Ellis, R.B. Dunlap, A.L. Pollard, K. Seidman, A.D. Cardin, Journal of American Chemical Society, 95 (1973) 4398.

[95] R.L. Lipnick, John D. Fissekis, Journal of Organic Chemistry, 44, (1979) 1627. 
[96] R.F. Stewart, Acta Crystallographica, 23 (1967) 1102.

[97] G. Wu, S. Dong, R. Ida, N. Reen, Journal of Americal Chemical Society, 124 (2002) 1768.

[98] S. Chandrasekaran, W.D. Wilson, D.W. Boykin, Journal of Organic Chemistry, 50 (1985) 829.

[99] E. Bednarek, J.Cz. Dobrowolski, K. Dobrosz-Teperek, L. Kozerski, W. Lewandowski, A.P. Mazurek, Journal of Molecular Structure, 554 (2000) 233.

[100] B. Blicharska, T. Kupka, Journal of Molecular Structure, 613 (2002) 153.

[101] Coutinho K.; Canuto, S.; DICE, a Monte Carlo program for molecular liquid simulations, University of São Paulo, São Paulo, Brazil, 2003.

[102] W.L. Jorgensen, J. Chandrasekhar, J.D. Madura, R.W. Impey, and M.L. Klein, Journal of Chemical Physics, 79 (1983) 926.

[103] C.M. Breneman, K.B. Wiberg, Journal of Computational Chemistry, 11 (1990) 361.

[104] T.W. Keal, D.J. Tozer, Journal of Chemical Physics, 121 (2004) 5654.

[105] A.A. Auer, Chemical Physics Letters, 467 (2009) 230.

[106] (a) A.D. Becke, Journal of Chemical Physics 98 (1993) 5648. (b) C. Lee, W. Yang, R.G. Parr, Physical Review B, 37 (1988) 785.

[107] C. Adamo, V. Barone, Journal of Chemical Physics A, 110 (1999) 6158.

[108] T.W. Keal, T. Helgaker, P. Salek, D.J. Tozer, Chemical Physics Letters, 425 (2006) 163.

[109] C. Adamo, V. Barone, Journal of Chemical Physics, 108 (1998) 664.

[110] (a) J. P. Perdew, in Electronic Structure of Solids '91, Ed. P. Ziesche and H. Eschrig (Akademie Verlag, Berlin, 1991) 11. (b) J. P. Perdew, J.A. Chevary, S. H. Vosko, K. A. Jackson, M. R. Pederson, D. J. Singh, C. Fiolhais, Physical Review B, 46 (1992) 6671. (c) J.P. Perdew, J.A. Chevary, S.H. Vosko, 
K.A. Jackson, M.R. Pederson, D.J. Singh, C. Fiolhais, Physical Review B, 48 (1993) 4978. (d) J.P. Perdew, K. Burke, Y. Wang, Physical Review B, 54 (1996) 16533. (d) K. Burke, J.P. Perdew, Y. Wang, in Electronic Density Functional Theory: Recent Progress and New Directions, Ed. J. F. Dobson, G. Vignale, and M. P. Das (Plenum, 1998).

[111] R. Wysokiński, D. Michalska, Journal of Computational Chemistry, 22 (2001) 901.

[112] D. Michalska, R. Wysokinski, Collection of Czechoslovak Chemical Communications, 69 (2004) 63.

[113] W.P. Ozimiński, P. Garnuszek, E. Bednarek, J.Cz. Dobrowolski, Inorganica Chimica Acta, 360 (2007) 1902.

[114] F. Jensen, Journal of Chemical Theory and Computational, 4 (2008) 719.

[115] K. Wolinski, J.F. Hinton, P. Pulay, Journal of American Chemical Society, 112 (1990) 8251.

[116] J. Gauss, Journal of Chemical Physics, 99 (1993) 3629.

[117] R.B. Brown, P.D. Godfrey, D. Mc Naughton, A.P. Pierlot, Journal of American Chemical Society, 110 (1989) 514.

[118] M.J. Frisch, et al,; Gaussian 03, Revision D 02, Gaussian:Wallingford, 2004.

[119] R.E. Wasylishen, D.L. Bryce, Journal of Chemical Physics, 117 (2002) 22.

[120] S. Millefiori, A. Alparone, Chemical Physics, 303 (2004) 27.

[121] V. Manzoni, M.L. Lyra, R.M. Gester, K. Coutinho, S. Canuto, Physical Chemistry Chemical Physics, 12 (2010) 14023.

[122] G. Sánchez-Sanz, I. Alkorta, C. Trujillo, J. Elguero, Tetrahedron, DOI:10.1016/j.tet.2012.05.056.

[123] S.B. Chattaraj, K. Sharma, A. Chakrabortty, S.C. Lahiri, Spectrochimica Acta Part A: Molecular and Biomolecular Spectroscopy, http://dx.doi.org/10.1016/j.saa.2012.04.069.

[124] M. Majumder, N. Sathyamurthy, Theoretical Chemistry Accounts, 131 (2012) 1092. 
[125] J. Fotie, H.V.K. Wangun, K. Dreux, T. Sommerfeld, J. Pittman, Magnetic Resonance in Chemistry, 50 (2012) 68.

[126] J.C. Facelli, Chemical Physics Letters, 322 (2000) 91.

[127] A. Wiersma, E.J.A.X. van de Sandt, M.A. den Hollander, H. van Bekkum, M. Makkee, J.A. Moulijn, Journal of Catalysis, 177 (1998) 29.

[128] C.M. Araujo, M.D. Doherty, S.J. Konezny, O.R. Luca, A. Usyatinsky, H. Grade, E. Lobkovsky, G.L. Soloveichik, R.H. Crabtree, V.S. Batista, Dalton Transactions, 41 (2012) 3562.

[129] J.A. Hatnean, S.A. Johnson, Organometallics, 31 (2012) 1361.

[130] H. Hirao, Y. Nagae, M. Nagaoka, Chemical Physics Letters, 348 (2001) 350.

[131] N. Okuyama Yoshida, K. Kataoka, M. Nagaoka, T. Yamabe, Journal of Chemical Physics, 113 (2000) 3519.

[132] N. Okuyama-Yoshida, M. Nagaoka, T. Yamabe, International Journal of Quantum Chemistry, 70 (1998) 95.

[133] G.G. Broyden, IMA Journal of Applied Mathematics, 6 (1970) 76.

[134] R. Fletcher, Computer Journal, 13 (1970) 317.

[135] D. Goldfarb, Mathematics of Computation, 24 (1970) 23.

[136] D.F. Shanno, Mathematics of Computation, 24 (1970) 647.

[137] D.F. Shanno, P.C. Kettler, Mathematics of Computation, 24 (1970) 657.

[138] H.C. Georg, S. Canuto (2009) Diceplayer, University of São Paulo, São Paulo, Brazil.

[139] R.M. Gester, H.C. Georg, S. Canuto, M.C. Caputo, P.F. Provasi, Journal of Physical Chemistry, 113 (2009) 14936.

[140] M.E. Martin, M.L. Sánchez, F.J. Oliveira del Valle, M.A. Aguilar, Journal of Chemical Physics, 113 (2000) 6308.

[141] I.F. Galván, M.L. Sánchez, M.E. Martin, F.J. Olivares del Valle, M.A. Aguilar, Journal of Chemical Physics, 118 (2003) 255.

[142] O. Christiansen, K.L. Bak, H. Koch, S.P.A. Sauer, Chemical Physics Letters, 284 (1998) 47. 
[143] P.F. Provasi, G.A. Aucar, S.P.A. Sauer, Journal of Chemical Physics, 115 (2001) 1324.

[144] DALTON, a molecular electronic structure program, Release Dalton2011 (2011).

[145] A.D. Becke, Journal of Chemical Physics, 98 (1993) 5648.

[146] C. Lee, W. Yang, R.G. Parr, Physical Review B, 37 (1988) 785.

[147] B.B DeMore, W.S. Wilcox, J.H. Goldstein, Journal of Chemical Physics, 22 (1954) 876.

[148] D.R. Lide, Handbook of Chemistry and Physics, CRC-Press, Boca Raton, 73rd edn, 1992-1993, 1992.

[149] E.E. Fileti, K. Coutinho, T. Malaspina, S. Canuto, Physical Review E, 67 (2003) 061504.

[150] T. Malaspina, K. Coutinho, S. Canuto, Journal of Chemical Physics, 117 (2002) 1692.

[151] R.F. Ribeiro, A.V. Marenich, C.J. Cramer, D.G. Truhlar, Journal of Chemical Theory and Computation, 5 (2009) 2284.

[152] F.H. Stilinger, A. Rahman A, Journal of Chemical Physics, 60 (1974) 3336.

[153] M. Mezei, D.L. Beveridge, Journal of Chemical Physics, 74 (1981) 622.

[154] R.H. Contreras, J.E. Peralta, Progress in Nuclear Magnetic Resonance Spectroscopy, 37 (2000) 321.

[155] J. Kongsted J, B. Mennucci, Journal of Physical Chemistry A, 111 (2007) 9890.

[156] R.M. Claramunt, M. Perez-Torralba, D.S. Maria, D. Sanz, B. Elena, I. Alkorta, J. Elguero, Journal of Magnetic Resonance, 206 (2010) 274279.

[157] I. Alkorta, J. Elguero, Chemical Physics Letters, 489 (2010) 159.

[158] I. Alkorta, J. Elguero, H.H. Limbach, I. G.Shenderovich, T. Winkler, Magnetic Resonance in Chemistry, 47 (2009) 585.

[159] I. Alkorta, F. Blanco, J. Elguero, Magnetic Resonance in Chemistry, 47 (2009) 249. 
[160] J.E. Del Bene, I. Alkorta, J. Elguero, Journal of Physical Chemistry A, 112 (2008) 6338.

[161] J.E. Del Bene, I. Alkorta, J. Elguero, Journal of Chemical Theory and Computation, 4 (2008) 967.

[162] I. Alkorta, J. Elguero, G.S. Denisov, Magnetic Resonance in Chemistry, 46 (2008) 599.

[163] J.E. Del Bene, I. Alkorta, J. Elguero, Magnetic Resonance in Chemistry, 46 (2008) 457.

[164] D. Sanz , R. M. Claramunt, A. Saini, V. Kumar , R. Aggarwal, S. P. Singh, I. Alkorta, J. Elguero, Magnetic Resonance in Chemistry, 45 (2007) 513.

[165] E.E. Fileti, H.C. Georg, K. Coutinho, S. Canuto, Journal of Brazilian Chemical Society, 18 (2007) 74.

[166] A. Salgado, C. Varela, A.M.G. Collazo, F. García, P. Pevarello, I. Alkorta, J. Elguero, Journal of Molecular Structure, 987 (2011) 13.

[167] R. Aggarwal, G. Sumran, R.M. Claramunt, D. Sanz, J. Elguero, Journal of Molecular Structure, 934 (2009) 96.

[168] Z.D. Pardo, G.L. Olsen, M.E. Fernández-Valle, L. Frydman, R. MartínezÁlvarez, A. Herrera, Journal of American Chemical Society, 134 (2012) 270.

[169] F.D. Rochon, H. Titouna, Inorganica Chimica Acta, 363 (2010) 1679.

[170] A. Herrera, R. Martínez-Alvarez, N. Martín, M. Chioua, R. Chioua, D. Molero, A. Sánchez-Vázquez, J. Almy, Tetrahedron, 65 (2009) 5817.

[171] A. Amera, S. Seniorab, X. Fanc, Nucleosides, Nucleotides and Nucleic Acids, 31 (2012) 42.

[172] M.P. Coles, M.S. Khalaf, R.M. Claramunt, M.A. García, I. Alkorta, J. Elguero, Journal of Physical Organic Chemistry, 23 (2010) 526.

[173] B. Puffer, C. Kreutz, U. Rieder, M.O. Ebert, R. Konrat, R. Micura, Nucleic Acids Research, 37 (2009) 7728.

[174] M. Chhabria, I. Rathod, K. Vala, P. Patel, Medicinal Chemistry Reserach, 9 (2011) 1450. 
[175] H. Yamada, K. Mizusawa, R. Igarashi, H. Tochio, M. Shirakawa, Y. Tabata, Y. Kimura, T. Kondo, Y. Aoyama, S. Sando, ACS Chemical Biology, 7 (2012) 535.

[176] K. Coutinho, S. Canuto, Advances in Quantum Chemistry, 28 (1997) 89.

[177] K. Coutinho, S. Canuto, Journal of Chemical Physics, 113 (2000) 9132.

[178] K. Coutinho, S. Canuto, THEOCHEM, 632 (2003) 235.

[179] B. Mennucci, Journal of American Chemical Society, 124 (2002) 1506.

[180] R. G. Parr e W. Yang, Density-Functional Theory of Atoms and Molecules, Oxford University Press, New York (1989).

[181] H. J. C. Berendsen, J. P. M. Postma, W. F. van Gusteren and J. Hermans, in Intermolecular Forces, ed. B. Pullman, Reidel, Dordrecht, 1981, p. 331.

[182] W.L. Jorgensen, N.A. McDonald, THEOCHEM, 424 (1998) 145.

[183] W.L. Jorgensen, J.M. Briggs, M.L. Contreras, Journal of Physical Chemistry, 94 (1990) 1683.

[184] M.L. Sánchez, M.A. Aguilar, F.J. Olivares del Valle, Journal of Computational Chemistry, 18 (1997 313.

[185] M.L. Sánchez, M.E. Martin, M.A. Aguilar, F.J. Olivares del Valle, Journal of Computational Chemistry, 21 (2000) 705.

[186] M.L. Sánchez, M.E. Martin, I. Fdez. Galvan, F.J. Olivares del Valle, M.A. Aguilar, Journal of Physical Chemistry B, 106 (2002) 4813.

[187] E. Cances, B. Mennucci, J. Tomasi, Journal of Chemical Physics, 107 (1997) 3031.

[188] M. Cossi, V. Barone, B. Mennucci, J. Tomasi, Chemical Physics Letters, 286 (1998) 253.

[189] J. Gao, K. Byun, Theoretical Chemistry Accounts, 96 (1997) 151.

[190] T. Ohba, S. Ikawa, Molecular Physics, 73 (1991) 985.

[191] D.C. Rapaport, Molecular Physics, 50 (1983) 1151.

[192] A. Møgelhøj, K. Aidas, K.V. Mikkelsen, S.P.A. Sauer, J. Kongsted, Journal of Chemical Physics, 130 (2009) 134508. 
[193] C. Reichardt, Chemical Review, 94 (1994) 2319.

[194] R.M. Gester, H.C. Georg, T.L. Fonseca, P.F. Provasi, S. Canuto, Theoretical Chemistry Accounts, 131 (2012) 1220.

[195] J. Kongsted, K. Ruud, Chemical Physics Letters, 451 (2007) 226.

[196] K. Coutinho, S. Canuto, Journal of Chemical Physics, 113 (2000) 9132.

[197] W.M. Litchman, M. Alei Jr., A.E. Florin, Journal of Chemical Physics, 50 (1969) 1031.

[198] R.A. Bernheim, G. Berthier, Theoretical Chimica Acta, 14 (1969) 71.

[199] R.E. Wasylishen, J.O. Friedrich, Canadian Journal of Chemistry, 65 (1987) 2238 .

[200] G.J. Jameson, A.K. Jameson, D. Oppusunggu, S. Wille, P.M. Burrel, J. Mason, Journal of chemical Physics, 74 (1981) 81.

[201] G.J. Jameson, A.K. Jameson, S.M. Cohen, H. Parker, D. Oppusunggu, P.M. Burrel, S. Wille, Journal of Chemical Physics, 74 (1981) 1608.

[202] B. Mennucci, J.M. Martınez, J. Tomasi, Journal of Physical Chemistry A, 105, (2001) 30.Klein, Chemical Physics Letters, 104 (1984) 579.

[203] K. Coutinho, M.J. Oliveira, S. Canuto, International Journal of Quantum Chemistry, 66 (1998) 249.

[204] R.W. Impey, M.L. Klein, Chem. Phys. Lett., 104 (1984) 579.

[205] J. Gao, X. Xia, T.F. George, Journal of Physical Chemistry, 97 (1993) 9241.

[206] F. Jensen, Journal of Chemical Theory and Computation, 2 (2006) 1360.

[207] M.D. Marshall, K.C. Igzi, J.S. Muenter, Journal of Chemical Physics, 107 (1997) 1035.

[208] T.S. Almeida, K. Coutinho, B.J.C. Cabral, S. Canuto, Journal of Chemical Physics, 128 (2008) 014506.

[209] M. Alei Jr, A.E. Florin, W.M. Litchman, J.F. O’Brien, Journal of Physical Chemistry, 75 (1971) 932. 
[210] R.C. Barreto, S. Canuto, Chemical Physics Letters, 496, (2010) 236.

[211] R.A. Bernheim, H. Batiz-Hernadez, Journal of Chemical Physics, 40 (1964) 3446.

[212] M. Alei Jr., A.E. Florin, W.M. Litchman, J.F. O'Brien, Journal of Physical Chemistry, 75 (1971) 932.

[213] M.I. Burgar, T.E. St. Amour, and D. Fiat, Journal of Physical Chemistry, 85 (1981) 502.

[214] B. Valentine, A. Steinschneider, D. Dhawan, M.I. Burgar, T. St. Amour, D. Fiat, International Journal of Peptide and Protein Research, 25 (1985) 26.

[215] E.N. Nikolova, F.L. Gottardo, H.M. Al-Hashimi, Journal of American Chemistry Society, 134 (2012) 3667.

[216] A.V. Afonin, D.V. Pavlov, I.A. Ushakov, N.A. Keiko, Magnetic Resonance in Chemistry, (2012), DOI 10.1002/mrc.3828.

[217] C. Aisenbrey, N. Pendem, G. Guichard, B. Bechinger, Org. Biomol. Chem., 10 (2012) 1440.

[218] A.L. Lieblein, M. Krämer, A. Dreuw, B. Fürtig, H. Schwalbe, Angewandte Chemie, (2012), DOI: 10.1002/anie.201200549.

[219] A. Pullman and H. Berthod, Theoretica Chimica Acta, 10 (1968) 461.

[220] C.C. Costain, J.M. Dowling, Journal of Chemical Physics, 32 (1960) 155.

[221] H. Ohtaki, Journal of Molecular Liquids, 103 (2003) 3.

[222] T. Kupka, I.P. Gerothanassis, I.N. Demetropoulos, Journal of Molecular Structure, 531 (2000) 143.

[223] A.D. Buckingham, Canadian Journal of Chemistry, 38 (1960) 300.

[224] A.D. Buckingham, T. Schaefer, W.G. Schneider, Journal of Chemical Physics, 32 (1960) 1227.

[225] A.E. Reed, F. Weinhold, Journal of Chemical Physics, 78 (1983) 4066.

[226] D. Parker, Phys. Rev. 91 (1991) 1441.

[227] A.D. Buckingham, Chem. Phys. Lett., 398 (2004) 1.

[228] A.D. Buckingham, P. Fischer, Chem. Phys., 324 (2006) 111. 